COUPLING, MEMBRANE CONDUCTANCE, AND ION CHANNEL MRNA PROFILES IN THE ESTABLISHMENT AND MAINTENANCE OF NETWORK ACTIVITY IN THE CRUSTACEAN CARDIAC GANGLION

A Dissertation
Presented to
The Faculty of the Graduate School
At the University of Missouri
In Partial Fulfillment
Of the Requirements for the Degree
Doctor of Philosophy

By

Kick, Daniel R. (MU-Student)

Dr. David J. Schulz, Dissertation Supervisor

May 2021 
The undersigned, appointed by the dean of the Graduate School, have examined the dissertation entitled COUPLING, MEMBRANE CONDUCTANCE, AND ION CHANNEL MRNA PROFILES IN THE ESTABLISHMENT AND MAINTENANCE OF NETWORK ACTIVITY IN THE CRUSTACEAN CARDIAC GANGLION

Presented by Kick, Daniel R. (MU-Student)

A candidate for the degree of

Doctor of Philosophy

And hereby certify that, in their opinion, it is worthy of acceptance.

Dr. David J. Schulz

Dr. Johannes Schul

Dr. Bing Zhang

Dr. Satish S. Nair 


\section{ACKNOWLEDGEMENTS}

My Ph.D., and the work herein, is a product of the David J. Schulz lab at the University of Missouri-Columbia.

First, I would like to thank Dr. David J. Schulz. Joining his lab is one of the best decisions I have made. I've learned from watching his approach to teaching and presenting difficult concepts - always going to the essence of a topic and building upon than foundation - and his insistence on not losing sight of the question being addressed for the methodology, experiments, or analysis being used. As a mentor, Dr. Schulz has given me the freedom to generate and pursue questions that might not come to fruition (and many didn't) so that I could learn how to ask questions instead of just answer them.

Though my time in the Schulz lab l've had the pleasure of working alongside people who have made me a better scientist and colleague through their example. This includes graduate students: Brian Lane (Ph.D. 2016), Jennifer Wekenborg (M.A. 2016), Cindy Kyi (Ph.D. 2018), Adam Northcutt (Ph.D. 2019), Sherryl Henderson, José Viteri, and Benton Berigan, post-doctoral researchers: Dr. Michael Gray and Dr. Joe Santin, and the undergraduates and post baccalaureate scholars I've worked with and gotten to know: Kelly Hiersche, Rody Kingston, Katyln Sullivan, Ayla Ross, Abby Beckerdite, and Carrie Davis-Hansson. I would like to recognize Dr. Brian Lane who got me started with the cardiac ganglion and Dr. Adam Northcutt with whom I collaborated and phenomenal laboratory technician, Virginia (Ginny) Garcia, who in addition to her official capacity keeps an eye out for everyone in the lab and always has an ear to lend.

I would also like to thank the faculty outside the lab who have fostered my growth as a researcher. Particularly those who have served on my committee: Dr. Johannes Schul, Dr. Bing Zhang, Dr. Satish S. Nair, and Dr. Lorin Milescu. Additionally, I would 
also like to thank Dr. Libby King and Dr. Kevin Middleton who helped me develop the quantitative skillset I now rely on and who have been generous with their time.

For two years of my training, I was supported by an NIH T32 training grant and I would also like to thank Dr. Mark Hannink and Debbie Allen who oversee it. I have also been supported though a teaching assistantship and am grateful to Dr. Gerald Summers for providing the animal physiology TAs the ability to revise and extend the laboratory curriculum. Moreover, I would like to thank Dr. Marco Navarro, Sherryl Henderson, José Viteri, and particularly Benton Berigan for their observations, thoughts, and hard work to improving the course.

I may not have ended up where I am if it were not for the many role models and mentors who welcomed me into their labs before I decided to pursue a Ph.D. This includes Dr. Laszlo Kovacs, Dr. Christian Lorson, Dr. Rahul Kanadia, and especially Dr. Diane Janick-Buckner and Dr. Brent Buckner who catalyzed my interest in research and challenged me to be a better scholar years before I worked with them.

Penultimately, I wish to thank my family for their continuous understanding, support, and love. I am forever grateful to my parents Beth and Bob Kick and my sister Laura Geringer and her husband Paul.

Finally, and most importantly, I must express my gratitude to my spouse Grace. I cannot put into words how much her support, encouragement, and reminders to not take things too seriously means to me and how grateful I am to have her in my life. 


\section{TABLE OF CONTENTS}

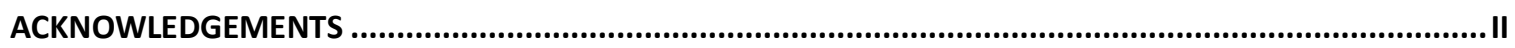

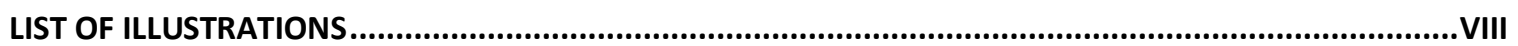

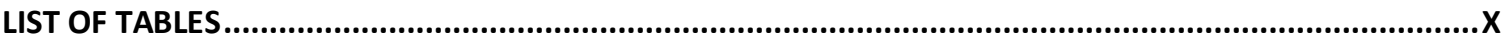

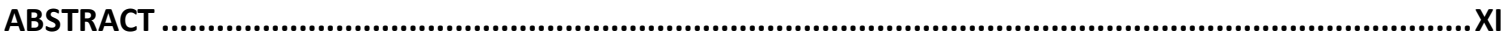

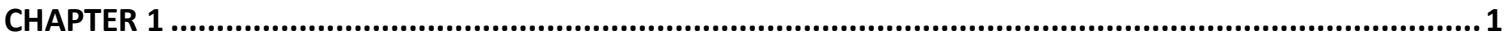

INTRODUCTION: PLASTICITY IN CELL AND NETWORK PROPERTIES WITHIN CENTRAL PATTERN

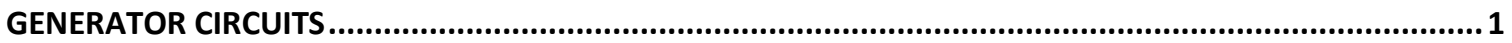

CHAPTER 2

TIMING DEPENDENT POTENTIATION AND DEPRESSION OF ELECTRICAL SYNAPSES CONTRIBUTES TO NETWORK STABILITY IN THE CRUSTACEAN CARDIAC GANGLION ..................................................

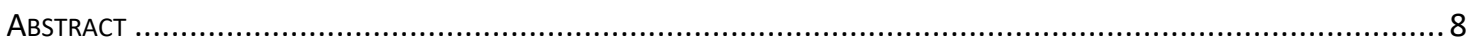

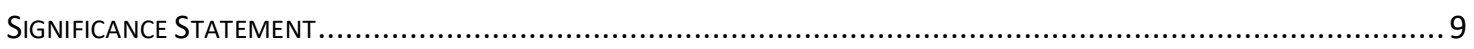

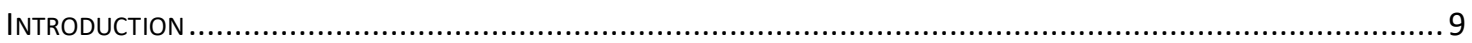

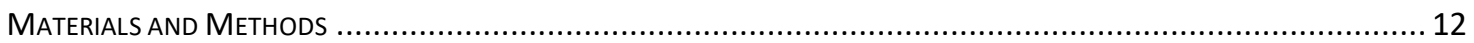

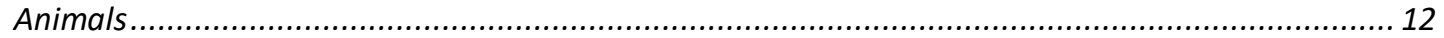

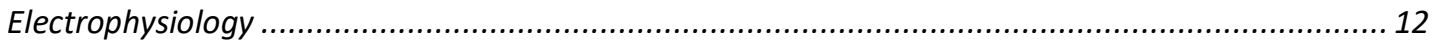

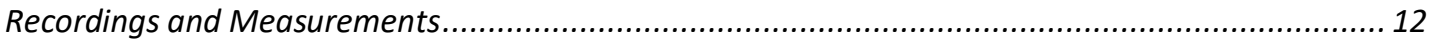

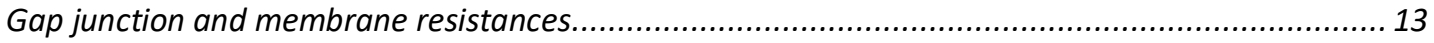

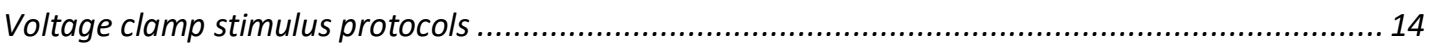

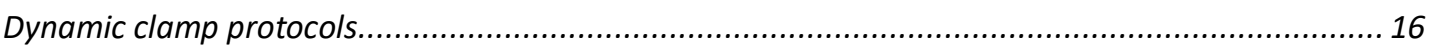

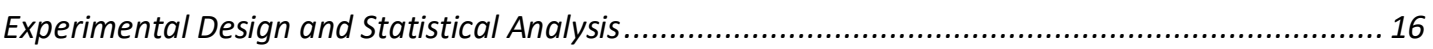

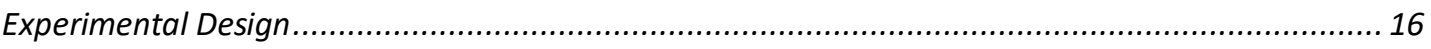

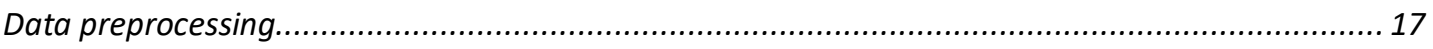

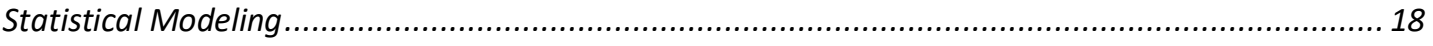

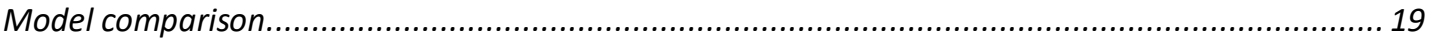

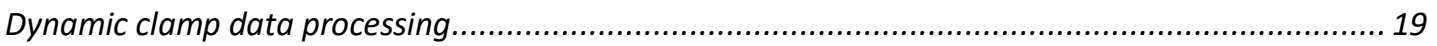

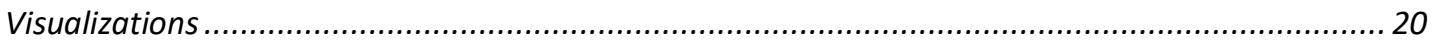

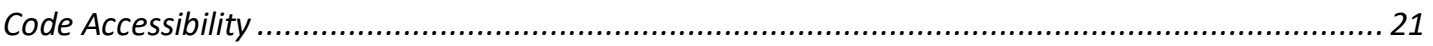

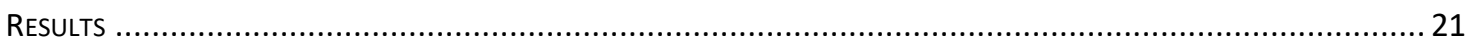

TEA mimetic protocol requires lack of synchrony and $\mathrm{Ca}^{2+}$ to increase coupling ............................... 21

A standardized control waveform increases or decreases coupling based on the delay between cells

Phase-shifted endogenous waveforms do not consistently alter coupling - Model comparison does not reveal a single better predictor than phase .............................................................................. 24

Dynamic Clamp reveals a detrimental effect of coupling strength and delay on cells' target output. 26

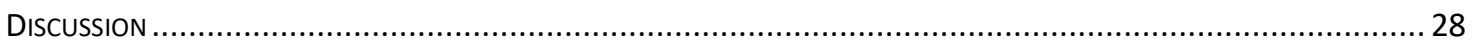

Loss of synchrony, and not increased depolarization, induces eLTP in LCS .................................... 29

The magnitude of phase difference may determine whether eLTP or eLTD is induced........................ 30

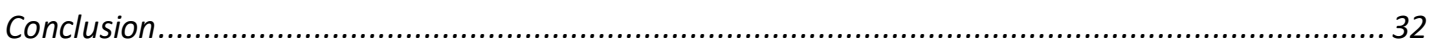

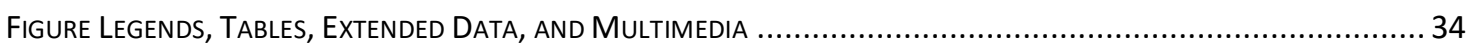

Figure 2.1 Synchronous, normative large cell (LC) depolarizations can be manipulated through

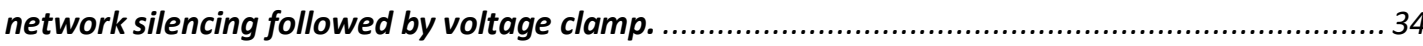


Figure 2.2 Electrical synapse potentiation (eLTP) requires desynchronized activity and $\mathrm{Ca}^{2+}$, not depolarization alone.

Figure 2.3 Desynchronization with a naturalistic stimulus below a $90^{\circ}$ phase shift depresses the $L C$ 4/5 electrical synapse, smaller delays potentiate the synapse.................................................. 37

Figure 2.4 Phase outperforms waveform derived metrics in explaining changes in coupling. ........ 38

Figure 2.5 LC output is altered by phase delay in a coupling conductance dependent manner...... 40

Figure 2.6 LC output is impaired by delay in a coupling conductance dependent manner .............. 42

CHAPTER 3

\section{EFFECTS OF BLOCKADE OF POTASSIUM CURRENTS ON MEMBRANE CONDUCTANCE AND CHANNEL EXPRESSION AT 1 HOUR AND 24 HOURS IN MOTOR NEURONS OF THE CARDIAC GANGLION ................ 52}

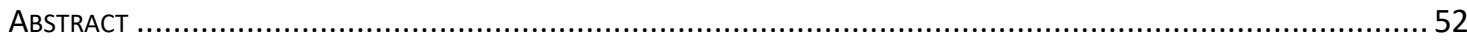

SIGNIFICANCE STATEMENT

INTRODUCTION

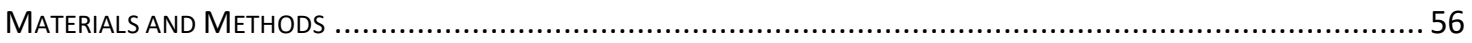

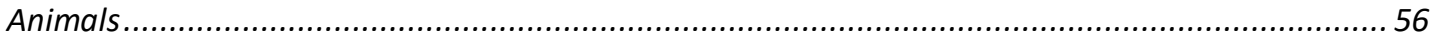

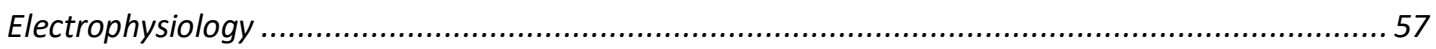

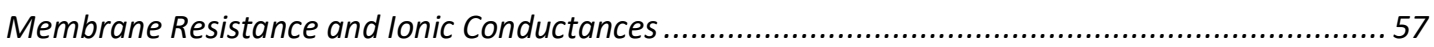

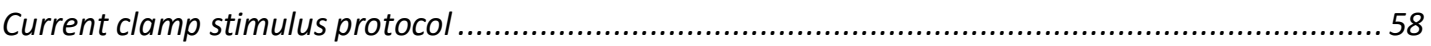

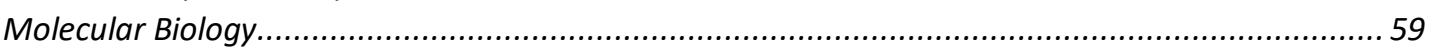

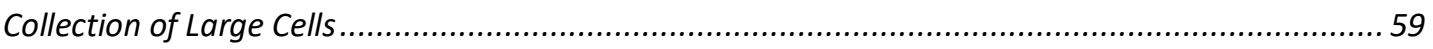

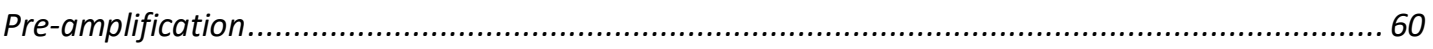

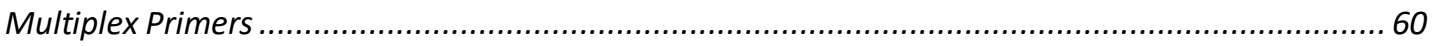

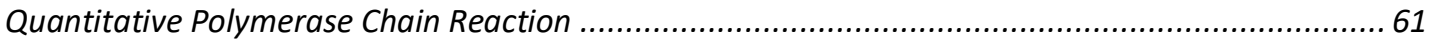

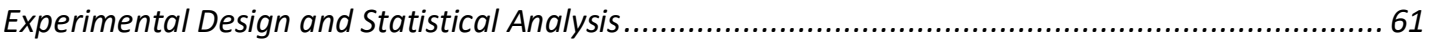

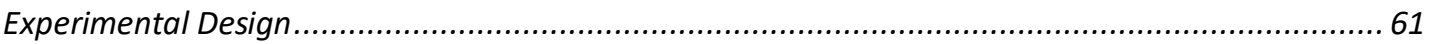

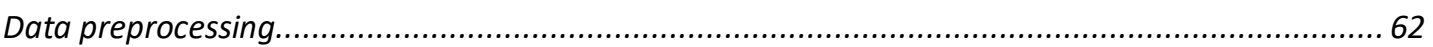

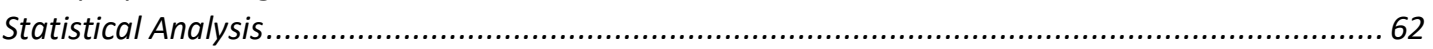

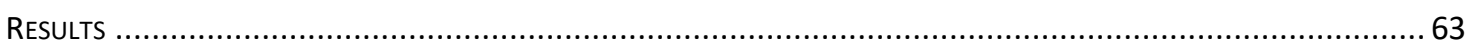

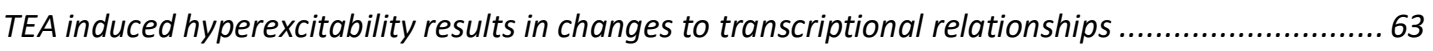

Transcript abundances reveal limited changes following TEA exposure...........................................6 66

Large cells do not maintain constant membrane properties over 24-hours of exposure.....................67 67

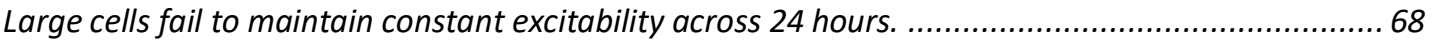

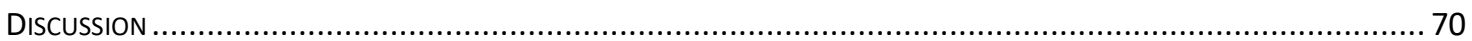

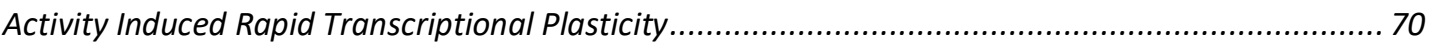

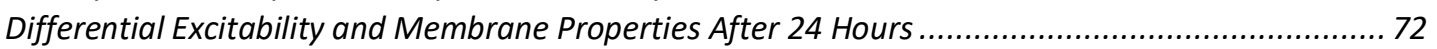

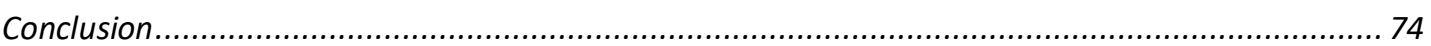

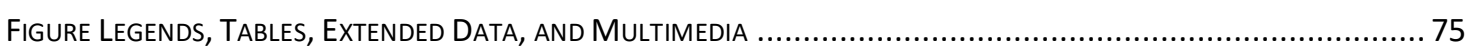

Figure 3.1 Increased Activity Alters and Strengthens Correlations ........................................... 75

Figure 3.2 Limited, Rapid changes in channel and gap junction mRNA abundances...................... 77

Figure 3.3 Excitability Metrics Are Inconsistent with Homeostasis at 24. .................................... 78

Figure 3.4 Plasticity in Membrane Properties at 24 Hours........................................................ 80

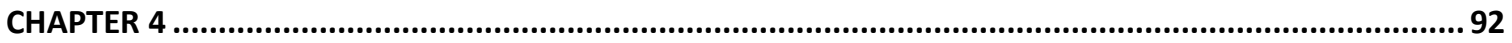

MOLECULAR PROFILING OF SINGLE NEURONS OF KNOWN IDENTITY IN TWO GANGLIA FROM THE CRAB

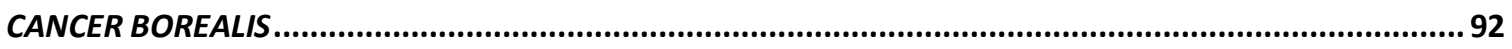

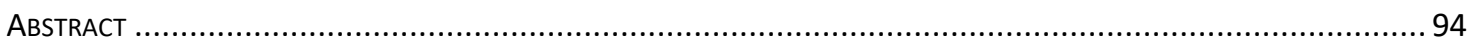

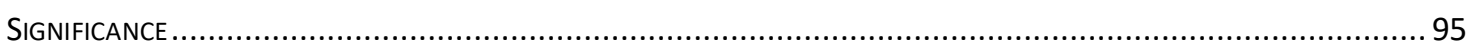




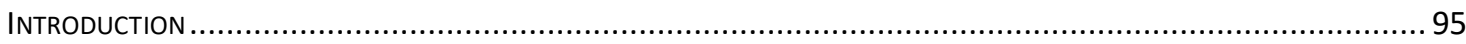

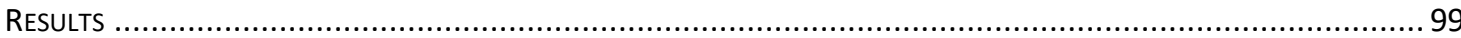

Molecular Profiling of Single Identified STG and CG Neurons by RNASeq..................................99

Principal Component Analysis of scRNA-Seq Datasets......................................................... 102

Gene Ontology Analyses of RNA-Seq Datasets..................................................................... 103

Molecular Profiling of Single Identified STG and CG Neurons Using Candidate Genes. ................... 104

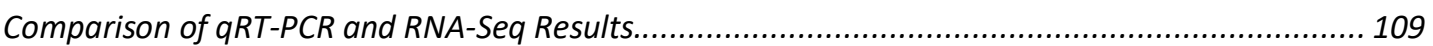

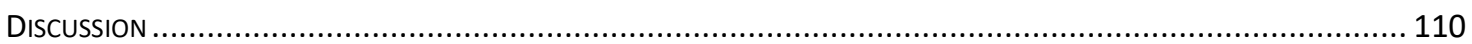

Physiological Insights into STG Network Function and Cell Identity. ....................................... 111

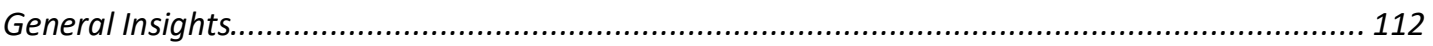

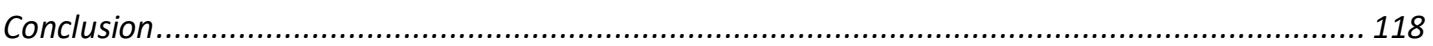

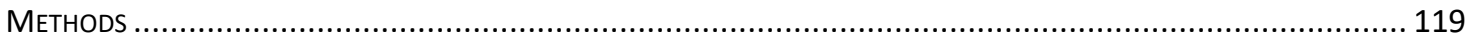

Cell Collection and RNA Preparation........................................................................ 119

Library Preparation and Single-Cell RNA-Seq........................................................... 120

MAPPING AND DIFFERENTIAL EXPRESSION. .................................................................................. 121

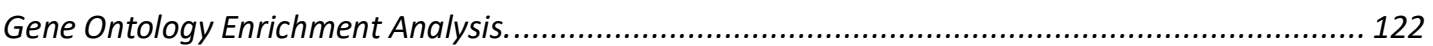

Multiplex Primer and Probe Design. .......................................................................... 122

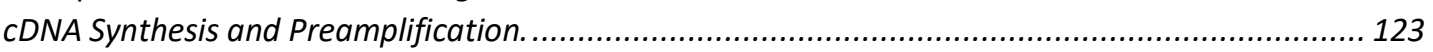

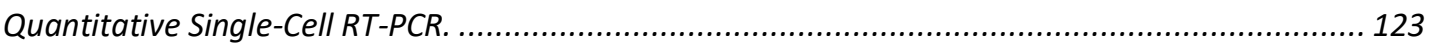

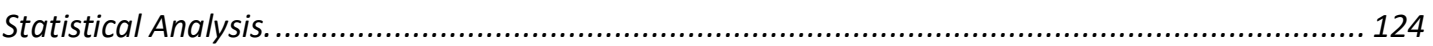

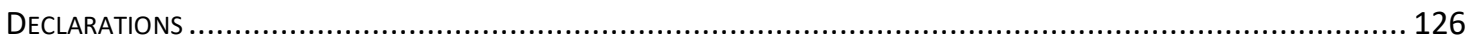

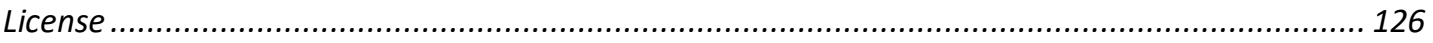

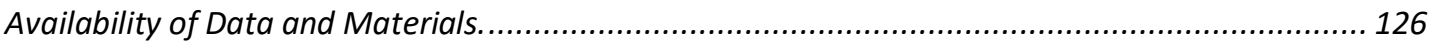

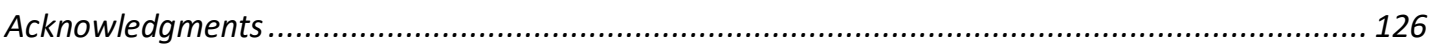

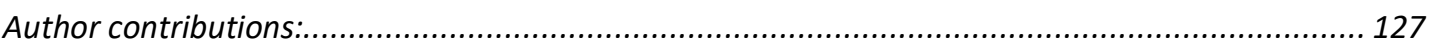

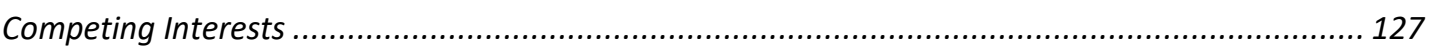

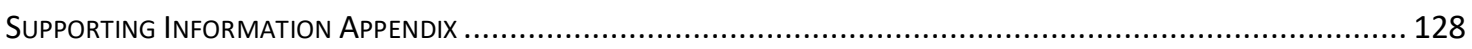

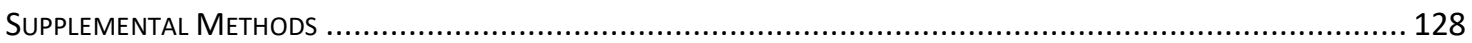

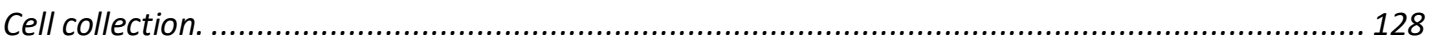

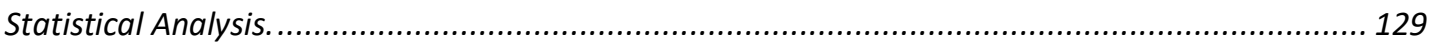

Figure LegendS, TABles, Extended DATA, ANd Multimedia ..................................................... 132

Figure 4.1 Connectome and representative waveforms of neurons from the stomatogastric and cardiac ganglia .......................................................................................................... 132 Figure 4.2 Efficacy of clustering, cluster estimation, and classification procedures with single-cell RNA-seq measurements....................................................................................... 134

Figure 4.3 Principal component analysis using variable or differentially expressed contigs ......... 136 Figure 4.4 Efficacy of clustering, cluster estimation, and classification procedures with single-cell

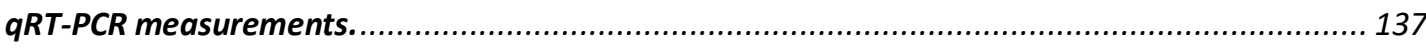

Figure 4.5 Expression level and clustering results using qRT-PCR or RNA-seq...................... 139

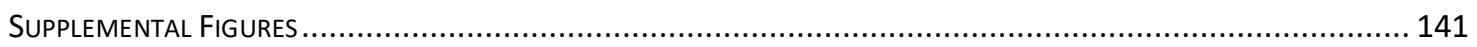

Figure 4.S1 Abundance of ion channels, innexins, and NA/K ATPase contigs ......................... 141

Figure 4.S2 Effect of preamplification of quantitation cycle ............................................ 142

CHAPTER 5

THE CANCER BOREALIS CARDIAC GANGLION AS A MODEL OF COMPENSATION: OPPORTUNITIES FOR

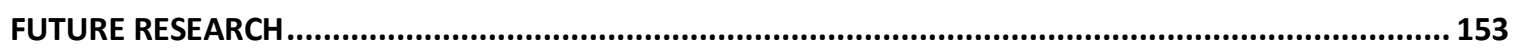

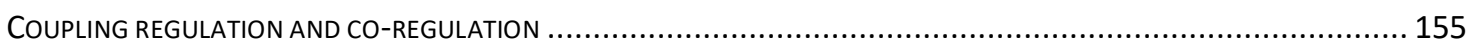

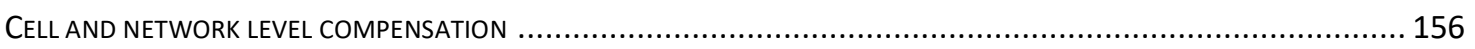




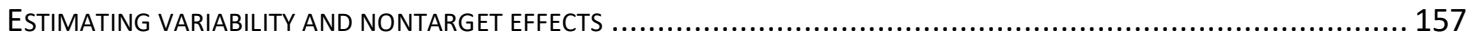

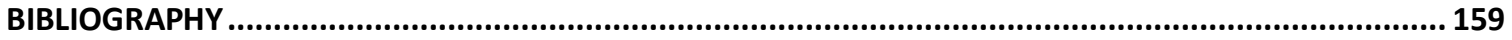

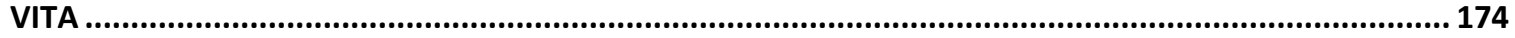




\section{LIST OF ILLUSTRATIONS}

Figure 2.1 Synchronous, normative large cell (LC) depolarizations can be manipulated through network silencing followed by voltage clamp.

Figure 2.2 Electrical synapse potentiation (eLTP) requires desynchronized

activity and $\mathrm{Ca}^{2+}$, not depolarization alone. 36

Figure 2.3 Desynchronization with a naturalistic stimulus below a $90^{\circ}$ phase shift depresses the LC 4/5 electrical synapse, smaller delays potentiate the synapse.

Figure 2.4 Phase outperforms waveform derived metrics in explaining changes in coupling. 38

Figure 2.5 LC output is altered by phase delay in a coupling conductance dependent manner.

Figure 2.6 LC output is impaired by delay in a coupling conductance dependent manner.

Figure 3.1 Increased Activity Alters and Strengthens Correlations. 75

Figure 3.2 Limited, Rapid changes in channel and gap junction mRNA abundances

Figure 3.3 Excitability Metrics Are Inconsistent with Homeostasis at 24. 78

Figure 3.4 Plasticity in Membrane Properties at 24 Hours. 80

Figure 4.1 Connectome and representative waveforms of neurons from the stomatogastric and cardiac ganglia.

Figure 4.2 Efficacy of clustering, cluster estimation, and classification procedures with single-cell RNA-seq measurements. 134

Figure 4.3 Principal component analysis using variable or differentially expressed contigs 136

Figure 4.4 Efficacy of clustering, cluster estimation, and classification procedures with single-cell qRT-PCR measurements. 
Figure 4.5 Expression level and clustering results using qRT-PCR or RNA-seq 139

Figure 4.S1 Abundance of ion channels, innexins, and NA/K ATPase contigs 141

Figure 4.S2 Effect of preamplification of quantitation cycle ............................. 142 


\section{LIST OF TABLES}

Table 2.1: TEA Mimic Sync \& TEA Mimic Async............................................ 44

Table 2.2: TEA Mimic Async \& Depolarized Async........................................ 46

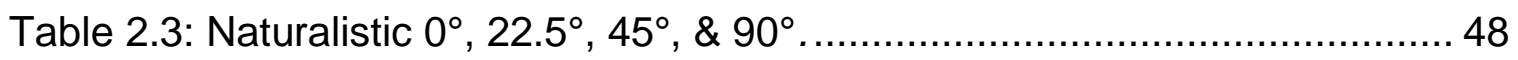

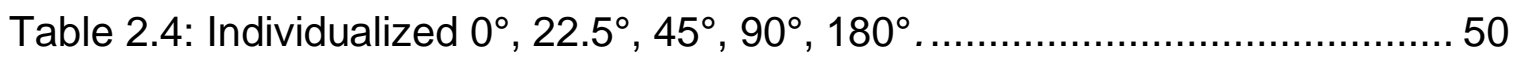

Table 3.1: Comparison of the Number Strong Correlations Across Replicates .. 81

Table 3.2: . Subset of Strong Correlations Observed Across Replications......... 82

Table 3.3: Bivariate transcript abundance relationships. ................................... 85

Table 3.4: Summary of statistical effects of treatment duration on measured

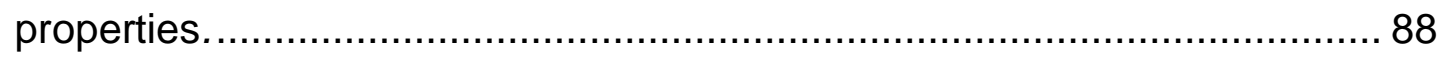

Table 3.S1: Multiplex qRT-PCR Primers Used.............................................. 91

Table 4.S1: Top ten contributing genes or contigs to PCs1-3 for each dataset.143

Table 4.S2: Gene Ontology Enrichment analysis of Molecular Function for H2K

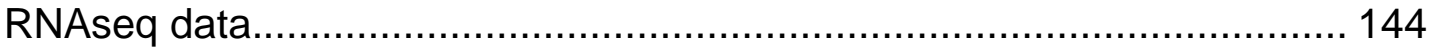

Table 4.S3: Gene Ontology Enrichment analysis of Biological Process for H2K RNAseq data. 145

Table 4.S4: Gene Ontology Enrichment analysis of Molecular Function for HVG RNAseq data

Table 4.S5: Gene Ontology Enrichment analysis of Biological Process for HVG RNAseq data 148

Table 4.S6: Gene Ontology Enrichment analysis of Molecular Function for DE0.2 RNAseq data. 149

Table 4.S7: Target primer and probe sequences for qRT-PCR Multiplex assays. 


\title{
COUPLING, MEMBRANE CONDUCTANCE, AND ION CHANNEL MRNA PROFILES IN THE ESTABLISHMENT AND MAINTENANCE OF NETWORK ACTIVITY IN THE CRUSTACEAN CARDIAC GANGLION
}

\author{
Daniel R. Kick \\ Dr. David J. Schulz, Dissertation Supervisor
}

\begin{abstract}
Neural networks produce critical rhythmic behaviors throughout an animal's lifespan, despite growth, differing environments, and changes in physiological state. This requires networks which balance stability in their properties with the plasticity necessary to respond to altered demands or perturbations. Studying the mechanisms which confer these properties requires a well characterized system with a known network topology and identifiable neurons that are amenable to both electrophysiological and molecular characterization and manipulation.
\end{abstract}

Here, we use two networks from Cancer borealis to explore activity dependent regulation of cell connectivity, changes in cell properties with prolonged perturbation, and reliability of gene expression as a means for cell identification. For the first two topics we use the cardiac ganglion alone. The cardiac ganglion consists of a kernel of four interneurons that drive five motor neurons (termed large cells, LCs) which innervate the heart musculature. LCs burst synchronously due to simultaneous stimulation and electrical coupling through gap junctions. Depolarizing pharmacological perturbations have been 
shown to result in hyperexcitability (Ransdell et al., 2012a) and disrupt synchrony between LCs (Lane et al., 2016) eliciting rapid plasticity in ionic currents and electrical coupling which restores synchrony and excitability (Ransdell et al., 2012a; Lane et al., 2016).

The salient electrophysiological signal which elicits coupling plasticity has not been identified. Using voltage clamp we directly control LC depolarizations to vary amplitude and timing of activity between LCs. We find that timing between cells, rather than depolarization elicits plasticity with the direction, i.e., potentiation or depression, being determined by the degree of desynchronization. With dynamic clamp we artificially couple networks from two animals and show that strong coupling with sufficient desynchronization can compromise a cell's output. These results suggest that coupling strength is tuned promoting synchrony or baseline cellular activity in a degree dependent manner.

While rapid compensatory plasticity to hyperexcitability has been shown, it is unknown whether the changes are solely post-transcriptional and whether the short-term changes persist over longer time scales. We perturb networks for one or twenty-four hours and compare LCs' excitability, membrane properties, and abundances of ion channel and gap junction transcripts. We find evidence of rapid transcriptional changes at one hour, which may be maintained or regress at twenty-four hours. Additionally, we find that membrane properties and excitability are not maintained from one to twenty-four hours, suggesting a failure to maintain homeostasis or that additional compensatory changes are occurring at the network level. 
To address our third topic, we use LCs in addition to neurons collected form the stomatogastric ganglion which coordinates mastication and filtering in the digestive track. Both systems allow for unambiguous identification of cells based on anatomy or neuronal projections. We use this to evaluate the efficacy of cluster estimation procedures, clustering methods, and classification algorithms to determine the number of cell types present, group like cells together, and identify cells based on gene expression alone. We use single cell RNA-seq and single cell qRT-PCR to measure all contigs or a select set of ion channel, receptor, and gap junction mRNAs. We find these methods do not reproduce the known number of cell types present. Furthermore, although clustering and classification both outperform chance, we are unable to recapitulate cell type with complete accuracy from these data. These results indicate that, while promising, determining cell type by molecular profiling should not be relied on as the sole metric of cell type determination. 


\section{Chapter 1}

\section{Introduction: Plasticity in cell and network properties within central pattern generator circuits}

Central pattern generator networks (CPGs) produce rhythmic behaviors essential for survival including chewing, locomotion, breathing, and in the case of Cancer borealis cardiac rhythmicity. These rhythms require coordination between the network's constituent neurons which in turn relies on individual neurons producing certain output. How neurons produce, maintain, and restore appropriate output is a key question for neuroscience to address.

Foundational research working to answer this question has relied on invertebrate CPGs. The systems used in this work, the cardiac ganglion and stomatogastric ganglion of $C$. borealis consist of nine (Cooke, 2002) and approximately twenty-six neurons respectively (Kilman and Marder, 1996). These systems allow unambiguous identification of neuronal cell type through anatomical location or nerve projections permitting identification of the same neuron across multiple animals. In the following studies we employ the cardiac ganglion as our primary model system and the stomatogastric ganglion as a secondary model.

The decapod cardiac ganglion varies in number and organization of its constituent neurons from species to species (Alexandrowicz, 1932; Cooke, 
2002). Cancer borealis, and many other species, possess four interneurons termed "small cells" which form a pacemaking kernel, and five motor neurons termed "large cells" (LCs) (Cooke, 2002). The ganglion consists of a central trunk with anterior and posterior bifurcations (Figure 2.1). The posterior region of the ganglion contains the SCs and a pair of LCs, LC 1, and LC 2. The left anterior branch of contains the sole unpaired LC, LC 3 with LC 4 and LC 5 occupying the right anterior branch. This asymmetric structure permits unambiguous identification of motoneurons. LCs burst simultaneously, synchrony being maintained through simultaneous excitation from the SCs and through electrical coupling between LCs. Electrical coupling between pairs of LCs is particularly strong with all LCs being more weakly coupled. Individual LCs can relatively easily be isolated and abundance of individual transcripts measured with single cell qRT-PCR. These features make the cardiac ganglion well suited to addressing questions related to coupling conductance and the plasticity thereof, compensation through transcriptional or post translational changes, and electrophysiological or molecular properties within a cell type. In the subsequent chapters we explore these three topics.

First, we examine electrical coupling and the salient signal which elicits activity dependent compensation thereof. Between electrically coupled neurons, current (Watanabe, 1958) and small molecules (Nakase and Naus, 2004) can pass directly from one cell to another. Gap junction proteins (innexins in invertebrates) assemble into hemichannels in the cell membrane which dock with hemichannels on an adjacent cell forming a pore (Oshima et al., 2016). Gap 
junctions are often considered to merely attenuate current, behaving in conjunction with the membrane as a low pass filter, but they may exhibit more complex behaviors including rectification (Furshpan and Potter, 1959; Haas et al., 2011a; Shruti et al., 2014) or band pass filtering (Curti and O'Brien, 2016) in conjunction with membrane conductances. In many cases gap junctions promote synchronization between coupled neurons as seen in the inferior olive (Long et al., 2002) although in certain circumstances strong coupling can facilitate desynchronization (Vervaeke et al., 2010).

Similar to chemical synapses (Turrigiano et al., 1998), gap junctions exhibit activity dependent plasticity. In the thalamic reticular nucleus, asymmetric current injections have been shown to induce long term depression of coupling (Haas et al., 2011b). Recent study of this system has suggested the direction of compensation, i.e. potentiation or depression, depends on the degree of calcium influx elicited (Fricker et al., 2020). Activity dependent modulation of gap junctions has also been observed in the Retizius cells of Hirudo medicinalis (Welzel and Schuster, 2018). Gap junction conductance is also subject to neuromodulation, e.g. by dopamine (Gerschenfelds, 1984).

Within the cardiac ganglion, comodulating of dopamine and serotonin prevents the decrease in synchronization seen with serotonin alone though increased coupling conductance between LCs (Lane et al., 2018). Tetraethylammonium (TEA) has been shown to decrease the similarity of LC voltage responses to excitatory post synaptic potential mimicking current injection protocols (Ransdell et al., 2013a) and synchronization in active 
networks (Lane et al., 2016). Dopamine induce coupling potentiation is sufficient to prevent desynchronization induced by TEA as well (Lane et al., 2018). Beyond neuromodulation induced plasticity, coupling potentiation has been implicated in the recovery of synchrony through an activity dependent mechanism following TEA induced hyperexcitability (Lane et al., 2016). Despite examples of activity dependent coupling plasticity across taxa (Haas et al., 2011a; Lane et al., 2016; Welzel and Schuster, 2018) the salient electrophysiological signal or signals that elicit this plasticity have not been identified. In chapter 2 we show evidence that the desynchronization between coupled neurons rather than hyperexcitability elicits plasticity. We further suggest that synaptic depression and potentiation balance synchrony and cell output by demonstrating that potentiation, while increasing synchrony between cells can compromise the output of an individual neurons if the difference in timing is too great.

The changes in coupling we observe, and the activity dependent changes induced by hyperexcitability through TEA previously studied, have focused on changes occurring within about one hour. In our next study we consider compensatory changes in the cardiac ganglion following one or twenty-four hours of TEA exposure and measuring changes in transcript expression in addition to membrane properties.

Changes in activity can result in remodeling of physiological and molecular relationships. One major line of inquiry has focused on the effects of eliminated activity. Stomatogastric neurons following loss of activity through dissociation (Turrigiano and Marder, 1993) or decentralization (Thoby-Brisson and Simmers, 
1998) regain the capacity for activity given several days in culture. The plasticity elicited appears to require changes in conductance magnitudes (Turrigiano et al., 1995) and transcription (Thoby-Brisson and Simmers, 2000). To determine the signal responsible for these changes, investigators have focused on the maintenance of baseline properties namely the correlated abundances of ion channel mRNAs (Schulz et al., 2007a) which have been verified to correlate with conductance for certain transcripts (Schulz, 2006). Although neuromodulation has been proposed to maintain these relationships, (Khorkova and Golowasch, 2007a), activity (Temporal et al., 2014a) - specifically voltage (Santin and Schulz, 2019a) appears to be the salient signal. This finding supports computational studies which suggest that intracellular calcium, acting as a proxy for activity, can lead to correlated ion channel expression (O'Leary et al., 2013a) similar to those observed in nature (O'Leary et al., 2014a).

Compensatory changes have been observed on a shorter time scale (i.e. approximately one hour rather than hours to days) in response to pharmacologically induced hyperexcitability. Blockade of the A-type potassium current $\left(I_{A}\right)$ with application of 4-aminopyridine or blockade of the high threshold potassium current $\left(\mathrm{I}_{\mathrm{HTK}}\right)$ with TEA results in hyperpolarization and rapid compensatory increase of $I_{H T K}$ or $I_{A}$ (Ransdell et al., 2012a). Due to variability in conductances between cells, TEA differentially effects LCs (Ransdell et al., 2013a) leading to loss of synchrony which is recovered through increased coupling (Lane et al., 2016). Whether increased activity disrupts LC ion channel mRNA correlations (Tobin et al., 2009) has not been examined. Furthermore, 
whether the rapid physiological changes observed represent a terminal state or whether these properties remain plastic over longer time periods has not been examined. In chapter 3 we address both questions disrupting LC activity for one or twenty-four hours and measuring excitability, membrane properties, and transcript abundances. We find that hyperexcitability induces rapid changes in transcription and that after twenty-four hours membrane properties differ from one hour and apparently fail to maintain compensation in excitability.

While the previous two questions have been fundamentally concerned with plasticity between cells or within a single cell type, our final study considers distinctions between cell types. The ability to unambiguously identify the same neuron across animals has revealed considerable variability. Within cardiac ganglion LCs ionic conductances have been shown to vary 2-4 fold (Ransdell et al., 2013a) and mRNA transcript abundances 3-9 fold (Tobin et al., 2009). Similar ranges, 2-4 fold in conductances (Schulz et al., 2006a) and 3-5 fold in mRNA transcript abundances (Schulz et al., 2007a) have been reported in cells of the stomatogastric ganglion. Variability has been noted in other systems as such as in dissociated mouse Purkinje neurons with of similar activities contain highly variable currents as well (Swensen, 2005). If the properties underlying a cell's behavior are highly variable this may confound determining a cell's type based on these features alone. This is of particular importance in systems without a known connectome and the capability to unambiguously identify neurons - which would permit validation of an expression-based means of determining cell type. In chapter 4 we use unambiguously identified neurons from the cardiac and 
stomatogastric ganglia to evaluate the effectiveness of machine learning methods to recapitulate neuronal cell type from gene expression. Using data from single cell RNA-seq to capture all contigs expressed or single cell qRT-PCR to measure abundances of ion channel and receptor mRNAs we find that common algorithms recapitulate neuronal cell better than expected by chance but imperfectly. Furthermore, tested procedures for estimating the number of clusters within a dataset did not arrive at the number of cell types present in the sample. We conclude that a multimodal approach remains necessary for determining cell identity. 


\title{
Chapter 2
}

\section{Timing dependent potentiation and depression of electrical synapses contributes to network stability in the crustacean cardiac ganglion}

\begin{abstract}
Central pattern generators produce many rhythms necessary for survival (e.g. chewing, breathing, locomotion) and to do so often requires coordination of neurons through electrical synapses. Because even neurons of the same type within a network are often differentially tuned, uniformly applied neuromodulators or toxins can result in uncoordinated activity. In the crab (Cancer borealis) cardiac ganglion, potassium channel blockers and serotonin cause increased depolarization of the five electrically coupled motor neurons as well as loss of normally completely synchronous activity. Given time, compensation occurs that restores excitability and synchrony, and one of the underlying mechanisms of this compensation is an increase in coupling among neurons. However, the salient physiological signal that initiates increased coupling has not been determined. Using male $C$. borealis, we show that it is the loss of synchronous voltage signals between coupled neurons, rather than overall depolarization, that is responsible for plasticity in coupling. Shorter offsets in naturalistic activity across a gap junction enhance coupling, while longer delays depress coupling. We also provide evidence as to why a desynchronization-specific potentiation or depression of the synapse could ultimately be adaptive by using a hybrid network created by artificially coupling two
\end{abstract}


cardiac ganglia. Specifically, a stray neuron may be "brought back" in line by increasing coupling if its activity is closer to the remainder of the network. However, if a neuron's activity is far outside network parameters, it is detrimental to increase coupling and therefore depression of the synapse removes a potentially harmful influence on the network.

\section{Significance Statement}

Understanding how neural networks maintain output over years despite environmental and physiological challenges requires understanding the regulatory principles of these networks. Here we study how cells that are synchronously active at baseline respond to becoming desynchronized. In this system, a loss of synchrony causes different parts of the heart to receive uncoordinated stimulation. We find a calcium-dependent control mechanism which alters the strength of electrical connections between motor neurons. While others have described similar control mechanisms, here we demonstrate that voltage changes are sufficient to elicit regulation. Furthermore, we demonstrate that strong connections in a sufficiently perturbed network can prevent any neuron from producing its target activity, thus suggesting why the connections are not constitutively as strong as possible.

\section{Introduction}

Electrical synapses are dynamic structures capable of activity-dependent plasticity (Haas Greenwald Pereda 2016) and reshaping circuit behavior (Marder et al., 2017; Alcamí and Pereda, 2019). Electrical synapses are integral to functions including escape behavior (Allen et al., 2006), visual encoding (Wang et al., 2017), and retinal processing (Kothmann et al., 2012). Electrical synapses undergo long term potentiation (eLTP) or depression (eLTD) due to changes in phosphorylation of the gap junction 
(Wang et al., 2015; Sevetson et al., 2017a) . Often metabotropic glutamatergic signaling through group 2 mGluR activation results in eLTP while group 1 activation results in eLTD (Wang et al., 2015).

The past decade has yielded evidence suggestive of a voltage-dependent modulation of electrical synapses. The thalamic reticular nucleus (TRN) undergoes eLTD or eLTP following evoked bursting (Haas et al., 2011c) or spiking (Fricker et al., 2020). Evoked spiking has been shown to cause eLTP in Hirudo medicinalis as well (Welzel and Schuster, 2018). The TRN results suggest a depolarization-dependent sign change, and it has been proposed that this is regulated via intracellular calcium (Sevetson et al., 2017a; Fricker et al., 2020). However, these experiments have not been conducted with mGluR antagonists and the complexity of the circuits involved make it challenging to disentangle potential voltage-dependent effects from metabotropic ones. Regardless, these studies provide compelling support for the possibility of a voltage dependent mechanism of ELTP and eLTD. In this study for the first time we address two provocative ideas put forward in this field in recent years: whether "the activity of electrical synapses themselves lead to their potentiation" (Haas et al., 2016) and whether "Activity-dependent LTD could be a mechanism used by a single bursting cell to unplug from ... [a] network" (Haas and Landisman, 2012) .

We address these questions using the Cancer borealis cardiac ganglion (CG) which contains five motor neurons (large cells, LCs) (Cooke, 2002) that depolarize synchronously (Figure 2.1A,B). Waveform synchrony occurs despite 2 to 4 fold variability in conductance magnitudes within LCs of a network (Ransdell, 2013a) through a tuning of membrane conductances (Lane et al., 2016), electrical coupling (Watanabe, 1958) and synchronous excitation. Pharmacological blockade of potassium channels with tetraethylammonium (TEA) results in hyperexcitability, increased activity (Ransdell et al., 
2012b), and desynchronized waveforms of these coupled cells (Ransdell et al., 2013). However, within 30-60 minutes of this loss of synchrony, increased coupling (eLTP) - in conjunction with increase in A-type potassium conductance - act to restore synchrony in a compensatory fashion (Lane et al., 2016). However, it is not clear whether asynchronous voltage across the electrical synapses, increased activity and depolarization, or both are the salient signals initiating the response that enhances coupling.

We hypothesized that voltage across the gap junction, coincident with depolarization, is a relevant electrophysiological signal for coupling modulation. Investigating these mechanisms requires decoupling the increased depolarization/excitability TEA causes from signal synchrony. We accomplish this experimentally using two-electrode voltage clamp to precisely and independently control the membrane voltage of coupled cells. After suspending network activity, cells are clamped to the same activity with or without a delay between them.

We first replicated the findings of the TEA block results (Lane et al., 2016) without pharmacology using protocols that mimic depolarization levels seen in TEA. We show that eLTP only occurs when asynchronous activity is present and that this phenomenon is calcium-dependent. Next, we demonstrated that increased depolarization is not necessary to alter coupling: at typical burst amplitudes, asynchronous activity elicits plasticity. Further, the sign of the change (i.e. potentiation or depression) is dependent on the delay between cells, with short delay resulting in eLTP and long delay resulting in eLTD. Finally, we use hybrid networks of two independent CGs with a simulated electrical synapse connecting one LC in each network to show a potential benefit for the induction of eLTP at lesser degrees of loss of synchrony and eLTD when loss of synchrony is more severe. 


\section{Materials and Methods}

\section{Animals}

Wild caught adult male Jonah crabs (Cancer borealis) were purchased from The Fresh Lobster Company (Gloucester, MA). Upon arrival, animals were housed in tanks maintained at $12^{\circ} \mathrm{C}$ until use. Crabs were anesthetized prior to use by cold exposure for thirty minutes. The heart was removed from the animal and microdissected to isolate the ganglion, which was pinned out in a Sylgard lined dish. Motor neurons were exposed by removing the connective sheath around them with a fine pin. During the dissection, the preparation was bathed in chilled physiological saline composed of $440 \mathrm{mM} \mathrm{NaCl}, 26$ $\mathrm{mM} \mathrm{MgCl}$, $13 \mathrm{mM} \mathrm{CaCl}, 11 \mathrm{mM} \mathrm{KCl}$, and $10 \mathrm{mM} \mathrm{HEPES}$ at a pH of 7.4-7.5. All chemicals were purchased from Fisher Scientific unless stated otherwise.

\section{Electrophysiology}

\section{Recordings and Measurements}

The cardiac ganglion in $C$. borealis consists of nine neurons (Figure 2.1A). Four interneurons, "small cells" (SCs), form a pacemaking kernel which excite the five "large cell" (LC) motor neurons in precise synchrony to generate action potentials that drive muscle contraction (Figure 2.1A,B). Paired LC somata are found in an anterior branch of the network (Figure 2.1A), and among these paired somata are strong local electrical synapses that are the focus of these studies (Lane et al., 2016, 2018). A petroleum jelly well around the posterior bifurcation isolated the small cells (Figure 2.1A) and a section of ganglionic trunk from the anterior LCs (i.e. LC3, 4, and 5; Figure 2.1A,B). This was used for recording network activity extracellularly with stainless steel electrodes connected to a differential AC amplifier (A-M Systems model 1700). Desheathed somata of LC4 and LC5 were recorded from using sharp electrodes filled with $3 \mathrm{M} \mathrm{KCl} \mathrm{(4-16}$ 
$M \Omega$ ). Following baseline recordings physiological saline around the small cells was displaced by isotonic sucrose $(750 \mathrm{mM})$ to suspend network activity as described previously (Lane et al., 2016). When isotonic sucrose failed to fully suppress activity tetrodotoxin was added to eliminate residual activity. When measurements of baseline activity were not required, networks were sometimes silenced by cutting the trunk of the network to immediately and irreversibly remove SC input from the LCs.

\section{Gap junction and membrane resistances}

Two-electrode current and voltage clamp protocols were used to measure input resistance $\left(R_{11}\right)$ and coupling resistance $\left(R_{c}\right)$, or the resistance across the electrical synapse. To measure these with current clamp we used sequential current injections (of sufficient length for the voltage to reach steady state) into both LCs. This protocol consisted of five (5) sweeps where one cell received $0 \mathrm{nA}(250 \mathrm{~ms}),-6 \mathrm{nA}(1500 \mathrm{~ms})$, and $0 \mathrm{nA}$ (2999 ms) and the other $0 \mathrm{nA}$ (2999 ms), -6 nA (1500 ms), and $0 \mathrm{nA}$ (250 ms). Initially $-2 \mathrm{nA}$ pulses were used, but $-6 \mathrm{nA}$ proved to offer better signal to noise ratio. From the resulting traces we calculated input resistance $\left(\mathrm{R}_{11}=\frac{\Delta \mathrm{V}_{1}}{\mathrm{I}_{1}}\right)$, membrane resistance $\left(R_{1}=\frac{R_{i n 1} \cdot R_{i n 2}-R_{12}^{2}}{R_{i n 2}-R_{12}}\right)$, transfer resistance $\left(R_{12}=\frac{\Delta V_{2}}{I_{1}}\right)$, coupling coefficient $\left(C_{12}=\frac{\Delta V_{2}}{\Delta V_{1}}\right)$, and coupling resistance $\left(R_{c}=\frac{R_{i n 1} \cdot R_{i n 2}-R_{12}^{2}}{R_{12}}\right)$, where subscript 1 and 2 denote the presynaptic and post synaptic cells respectively. Each of these were measured bidirectionally for a given pair of cells. These formulas are reported in (Bennett, 1966a).

To gain a second, more direct measure of coupling via voltage-clamp, one cell was held at $-60 \mathrm{mV}$ while the other was stepped to a test voltage. Specifically, we clamped one cell at $-60 \mathrm{mV}(250 \mathrm{~ms})$ then a test voltage ranging from $-80 \mathrm{mV}$ to -40 in increments of 5 $\mathrm{mV}$ (1000 ms), then holding the stepped cell to $-60 \mathrm{mV}$ and stepping the other. The -60 
$\mathrm{mV}$ step was excluded as it resulted in no transjunctional voltage. From this, coupling

conductance can be derived $\left(g_{j}=\frac{I_{2}}{V_{2}-v_{1}}\right)$ (Spray et al., 1979). The reciprocal of $g_{j}, g_{j}{ }^{-1}$, is coupling resistance. To differentiate between measurements from current clamp and those from voltage clamp these resistance of coupling measurements will be referred to as $R_{c}$ and $g_{j}^{-1}$, the former being from current clamp, the latter from voltage clamp. As expected, we found a close correspondence between $\mathrm{R}_{\mathrm{c}}$ and $\mathrm{g}^{-1}$ (data not shown). For experiments where both $\mathrm{g}_{j}^{-1}$ and $\mathrm{R}_{\mathrm{c}}$ were collected (i.e. all save those where endogenous waveforms were used.), we plot $g_{j}^{-1}$ rather than $R_{c}$, as the derivation thereof is more direct. No directionality or rectification was observed (data not shown), so measurements of $R_{c}$ and $g_{j}^{-1}$ from LC4 and LC5 are treated as pseudoreplicates.

The above protocols were created and run in Clampex 10.7 (Molecular Devices, San Jose CA) using two Axoclamp-2A intracellular amplifiers (Axon Instruments), a Brownlee precision amplifier (Model 410) for preamplification of the current injecting channels, and a Digidata 1440A digitizer (Molecular Devices).

\section{Voltage clamp stimulus protocols}

Following baseline measurements, LCs were voltage clamped to a waveform with increased depolarization or a naturalistic waveform. To mimic the effects of tetraethylammonium (TEA) on LC waveforms (Ransdell et al., 2013a; Lane et al., 2016), we created a TEA mimetic stimulus protocol (Figure 2.1C TEA Mimic) consisting of a high voltage, long duration pulse (depolarization to approximately $-18 \mathrm{mV}$, an increase of $35 \mathrm{mV}$ from a resting membrane potential of $-53 \mathrm{mV}$, for $1.61 \mathrm{Sec}$ ). This protocol was derived from a control LC waveform, where the depolarization was doubled in length and increased by $30 \mathrm{mV}$. Spike transients were removed to prevent excessive depolarization which left a shape more akin to an extreme TEA waveform. To mimic the TEA induced 
desynchronization reported previously (Ransdell et al., 2013a), we introduced a delay of $0.59 \mathrm{Sec}$ between the two voltage protocols (see Figure 2.2A, ASYNC).

The desynchronized TEA Mimetic protocol still contains overlap in the on phases between cells, resulting in a portion of the stimulus where there is no transjunctional voltage difference. Some TEA responses documented show little overlap in the depolarization phases of anterior LCs (i.e. LC3 vs LC4/5; see Figures 1E, 2A2, and 2A3 of (Ransdell et al., 2013a)). Therefore, we designed a protocol with shorter depolarizations which lacked overlap in the timing of the "on" phase between cells. In this "Depolarized protocol" (Figure 2.1C, 2.2B Depolarized), the on phase amplitude was consistent with the previous protocols ( $34 \mathrm{mV}$ from baseline) but remained elevated only for about 0.80 seconds, half as long as the depolarization in the previous protocols. Once again, we did not allow voltages above $-14 \mathrm{mV}$ to prevent excessive depolarization of spike transients. The delay between onset of cell depolarization was 0.98 seconds, $1 / 4$ the period, or $90^{\circ}$.

We then performed experiments with a treatment that more closely resembles natural, endogenous activity. To do so, we silenced preparations with isotonic sucrose and then either we used a standardized trace of natural control activity to serve as the voltage command for voltage-clamp protocols across preparations ("Naturalistic" protocol), or we created "Individualized" protocols where a network's waveform was recorded prior to silencing the network and then that network's own control activity used as a voltage-clamp command.

For asynchronous stimuli, a phase angle was introduced by shifting the voltage with respect to the time. When referred to here, "phase" is defined as a fraction of the period (e.g. a phase angle of $90^{\circ}$ for a cell with a 4 second period is equivalent to a 1 second delay). Representative phase angles used are visualized in Figure 2.1D (this 
example uses the Naturalistic waveform). These protocol manipulations were accomplished through a combination of operations in R (R Core Team, 2020) and Excel with the resultant time-voltage pairs saved as an axon text file. Stimulus protocols were used to drive LC4 and LC5 for one hour, with the above measurements being collected every 20 minutes and with each preparation being exposed to a single stimulus protocol type.

\section{Dynamic clamp protocols}

To assess the functional consequences of desynchronization between cells in an ongoing rhythm, we created a dynamic clamp protocol in Netclamp (Gotham Scientific) that simulated a bidirectional electrical synapse between LC3s in two cardiac ganglia. We then set the strength of this synapse to $0,0.025,0.05,0.1,0.15$, or $0.2 \mathrm{mS}$, consistent with conductances previously tested in this system (Lane et al., 2016). The simulated electrical synapse connected LC3 in two animals' cardiac ganglia (see Figure 2.5). Gap free recordings of the voltage in both cells, and extracellular recordings capturing small cell pacemaker activity, were collected from both networks and processed as described below. Note that while we compute the delay between burst onset, conversion to phase time is not appropriate here due to cycle-to-cycle variability in period.

\section{Experimental Design and Statistical Analysis}

\section{Experimental Design}

We designed the initial set of these experiments as a simple comparison of two groups; TEA mimetic synchronously applied vs applied with a delay. We repeated this design testing whether influx of calcium is necessary by using a high amplitude (although shorter duty cycle) protocol in the absence and presence of Cadmium Chloride 
$\left(250 \mu \mathrm{M} \mathrm{Cd}^{2+}\right)$. In the next set of experiments, we varied the delay between a single protocol using four phase angles: $0^{\circ}, 22.5^{\circ}, 45^{\circ}, 90^{\circ}$ (i.e. a delay of the $0,1 / 16,1 / 8$, or $1 / 4$, times the period). We performed a related set of experiments where rather than using a single protocol we allowed the protocol to vary while controlling the relative delay, this time with five phase angles: $0^{\circ}, 22.5^{\circ}, 45^{\circ}, 90^{\circ}, 180^{\circ}$.

Finally, we used dynamic clamp to vary the strength of fictive coupling between two cells of different networks at six levels $(0,0.025,0.05,0.1,0.15$, and $0.2 \mathrm{mS})$. We allowed delay between networks to vary as a second factor. Due to period variability within a network and between the two networks this factor is treated as being continuous. We sought two sets of networks such that one set possessed similar activity whereas the other set had mismatched activity. This final experiment is designed to be qualitatively, rather than quantitatively assessed.

None of experiments presented here were preregistered. No statistical procedure was used to determine sample size. Rather, we based the sample size on those efficacious for detecting similar effects in prior publications (e.g. (Ransdell et al., 2012b, 2013a; Lane et al., 2016)).

\section{Data preprocessing}

Measurements of voltage and current change or steps were obtained with Clampfit 10.7 and aggregated in Excel before being loaded into R (Wickham and Bryan, 2019) for analysis. As multiple measurements were taken at each timepoint the median values were retained for analysis. Data were quality controlled before modeling by ensuring all resistances were positive, coupling coefficient values were between 0 and 1 , and that all measured variables at each time and treatment were within two times the standard deviation above or below the mean. To eliminate pseudo replication in 
measurements of coupling resistance (i.e. $g_{j}^{-1}$ and $R_{c}$ ), the median of the gap junction resistance measures was calculated for each preparation at each time point and used in subsequent statistical modeling. This was not applied to coupling coefficient measures since they are free to vary between LCs due to independent membrane resistances. Data cleaning and processing libraries were fundamental to this process (Zeileis and Grothendieck, 2005; Müller, 2017; Vaughan and Dancho, 2018; Wickham et al., 2019; Firke, 2020) .

\section{Statistical Modeling}

Because our experimental design involves repeated measures over time, and multiple cells from the same individuals that share a synapse, and that quality control may result in unbalanced groups, we determined that a mixed model analysis - rather than ANOVA - would be most appropriate (McElreath, 2016). This allows for the starting value or level of a given dependent variable to be captured as a random effect to better examine the response to treatment over time. We modeled dependent variables as a linear function by main and interaction effects of time and phase using the library nlme (Pinheiro et al., 2020). The resultant models we evaluated with the aid of the car and broom libraries (Fox and Weisberg, 2019; Robinson and Hayes, 2020). We used a linear mixed effects model fit with restricted maximum likelihood to allow for an individual cell (or network in the case of $\mathrm{R}_{\mathrm{C}}$ and $\mathrm{g}_{\mathrm{j}}^{-1}$ ) to have an independent intercept but a shared slope. P values were corrected using a false discovery rate and are denoted as " $p_{\text {adj" }}$. Given the interrelated nature of our measurements (e.g. Input resistance being inextricably linked to membrane and gap junction resistance) our test results are not all independent. Therefore, applying an adjustment errs on the side of being a fairly conservative correction. As a companion to the above we have performed Kruskal-Wallis or Wilcoxon rank sum tests on coupling resistance measures. Unlike the linear models 
described previously, these non-parametric tests do not provide parameter estimates and thus do not act as a full substitute for the mixed model. However, they provide a more direct and intuitive test for evaluating experimental effects. We performed these tests on the change in coupling resistance from baseline at a single time point (40 minutes) for the TEA Mimetic, Naturalistic, and Individualized protocols. The 40 minute time point largely captures the steady state of the effects we observed. We have also used a Test of One-Sided Significance (TOST) (Robinson, 2016) to evaluate if $\mathrm{Cd}^{2+}$ application prevented voltage evoked potentiation. The resultant $p$ values for these were not adjusted in text.

\section{Model comparison}

We leveraged the protocol variability inherent in the individualized protocols to test if a single descriptor of the protocol (e.g. depolarization as represented as area under the curve) could outperform phase. Using custom functions, we measured the following from each protocol: minimum $\mathrm{mV}$, median $\mathrm{mV}$, mean $\mathrm{mV}$, maximum $\mathrm{mV}$, Area under the curve (AUC) (i.e. total depolarization), Overlapping AUC (i.e. AUC based on the minimum voltage between LCs), normalized joint AUC (i.e. overlapping AUC normalized to period), correlation between protocols, On duration, duty cycle, and percent delay. Additionally, we tested phase as a categorical variable. The same model structure as above was used, allowing fixed effects of treatment, time, and an interaction between them with a random effect of the preparation. These models were fit with maximum likelihood to enable model comparison with Akaike information criteria corrected (AICc, which corrects for the number of terms in the model) which was accomplished via the AICcmodavg library (Mazerolle, 2019).

\section{Dynamic clamp data processing}


Our goal with these dynamic clamp experiments was to measure the similarity of a burst to an idealized control burst and the similarity of a burst in one cell is to that of a coupled cell. We extracted the relevant voltage traces using the readABF library (Syekirin, 2018) and used custom R functions to find all instances where both networks were active simultaneously (henceforth termed an event). The start and end of an event was defined based on small cell spiking on the extracellular recordings. Since control cardiac ganglion waveforms are variable within and between animals one cannot align a burst to reference control, subtract the two and arrive at a robust estimate of the similarity to control bursts as a whole. To overcome this, we aligned each event to every control event and calculated the Pearson's correlation coefficient for the voltage of these bursts. We retain the median from each set to serve as the deviation from control activity for a given event. This is visualized in Figure 2.5 and in the graphs labeled AvA' and BvB' in Figure 2.6. To measure the similarity between bursts in two coupled cells (in this case belonging to different networks) we calculated the Pearson's correlation coefficient between the cells' voltages for an event. These values are shown in Figure 2.6 in the graphs labeled AvB and BvA.

We visualized the resultant data in two and three dimensions with respect to the delay in burst onset (as determined by the start of small cell bursting) between networks. We paired two networks such that one experiment contained cells of substantially differing burst amplitudes the other being of approximately matched amplitudes. This permits qualitative assessment of the effect of changes in onset independent of changes to both onset and depolarization.

\section{Visualizations}

We relied on a combination of R plotting libraries (Sievert, 2018; Edwards, 2019; Pedersen, 2019; Wilke, 2019; Slowikowski, 2020) to visualize the data produced for 
these experiments. Minor aesthetic adjustments were made with Adobe illustrator (Adobe Inc., 2019).

\section{Code Accessibility}

Both the data and code associated with these analyses are available upon request. Please contact the corresponding author.

\section{Results}

TEA mimetic protocol requires lack of synchrony and $\mathrm{Ca}^{2+}$ to increase coupling

Tetraethylammonium (TEA) application in known to increase LC depolarization and decrease network synchrony (Lane et al., 2016) in cardiac ganglia. These findings became the foundation for our first set of experiments, where we tested whether increased depolarization of the stimulus sufficed to change coupling, or if desynchronization also was necessary. The group exposed to the desynchronized TEA mimetic protocol exhibited a rapid decrease in coupling resistance (an increase in coupling strength) whereas the synchronous group did not (Figure 2.2A). We ran a pairwise test on coupling resistance and the linear model described previously on all dependent variables listed in table 2.1 to provide a more nuance description of the system. At 40 minutes the change in coupling between the two groups was significantly different $\left(\Delta \mathrm{gj}^{-1}\right.$ by Condition, $\mathrm{df}=9, \mathrm{~W}=29, \mathrm{p}=0.009$, Wilcoxon rank sum). In our linear model differences in the change between groups is described by an interaction effect on $\mathrm{g}_{j}{ }^{-1}\left(\mathrm{~g}^{-1}\right.$ Time:Condition $=\mathrm{F}(1,32)=4.781, \mathrm{p}=0.036, \mathrm{p}_{\mathrm{adj}}=0.084$, Linear MixedEffects), also appearing in our current clamp measurements of the resistance of coupling $\left(R_{c}\right.$ Time:Condition $=F(1,33)=3.809, p=0.060, p_{a d j}=0.095$, Linear Mixed-Effects $)$. We report $p$ adjusted for multiple comparisons $\left(p_{\text {adj }}\right.$ ) but this adjustment does not 
account for the relationships between the response variables (see methods). We also detected an increase in coupling coefficient in the desynchronized group (CC Time:Condition $=F(1,84)=9.027, p=0.004, p_{a d j}=0.012$, Linear Mixed-Effects $)$, estimated by the model to be $0.002\left(\Delta \mathrm{V}_{2} / \Delta \mathrm{V}_{1}\right) / 20 \mathrm{Min}$ in the asynchronous group. This Time:Condition effect on coupling coefficient appears to be driven by both a decrease in resistance of coupling and an increase in membrane resistance of $0.023 \mathrm{M} \Omega / 20 \mathrm{Min}\left(\mathrm{R}_{1}\right.$ Time:Condition $=F(1,44)=5.681, p=0.022, p_{a d j}=0.058$, Linear Mixed-Effects). Noninteraction effects and a full list of the tested variables are provided in Table 2.1.

To determine if the prolonged duration of the stimulus is necessary to alter coupling, we shortened the depolarization of the TEA Mimetic protocol above to match the length of a control burst. We maintained the high amplitude and asynchrony in this "Depolarized" protocol (Figure 2.1C). We noted similar changes in coupling relative to the TEA Mimetic despite halving the length of the depolarization (Figure 2.2B). When we visually compared the asynchronous Depolarized and TEA Mimetic protocols, we found both showed increased coupling (Figures 2.2A, 2.2B). We cannot conclude these groups significantly equivalent at 40 minutes, $\left(\Delta \mathrm{g}^{-1}\right.$, Condition at 40 minutes, $\mathrm{df}=8, \mathrm{p}=0.132$, Test of One-Sided Significance (TOST)) we also are unable to conclude they differ $\left(\Delta \mathrm{g}_{\mathrm{j}}^{-1}\right.$ by Condition, $\mathrm{df}=8, \mathrm{~W}=15, \mathrm{p}=0.691$, Wilcoxon rank sum). Similarly, the linear model does not suggest an interaction. This was the case both for direct $\left(\mathrm{g}_{\mathrm{j}}{ }^{-1}\right.$ Time:Condition $=$ $F(1,29)=0.002, p=0.969, p_{a d j}=0.969$, Linear Mixed-Effects $)\left(R_{c}\right.$ Time: Condition $=F$ $(1,29)=0.008, p=0.928, p_{\text {adj }}=0.963$, Linear Mixed-Effects $)$ and functional measures of coupling. None of the other parameters measured resulted in a substantial interaction effect (See Table 2.2).

To determine if the observed increase in coupling is dependent on calcium influx through voltage-dependent channels, we applied the Depolarized asynchronous protocol 
in the presence of extracellular $250 \mu \mathrm{M} \mathrm{CdCl}$. Blockade of calcium influx during the stimulus protocol virtually eliminated the increase in coupling seen as a result of the asynchronous Depolarized protocol (Figure 2.2B). At 40 minutes the coupling resistance of cells within the cadmium exposed networks (i.e. Depolarized async $+\mathrm{Cd}^{2+} 0$ minutes vs 40 minutes, Figure $2.2 \mathrm{~B}$, right panel) were significantly equivalent to their baseline values $\left(g_{j}{ }^{-1}, 0\right.$ vs 40 minutes, $d f=3, p=0.012$, Paired Test of One-Sided Significance (TOST)).

\section{A standardized control waveform increases or decreases coupling based} on the delay between cells

To test if these findings hold with a more naturalistic treatment, We used a stimulus protocol derived from a single control LC waveform applied at four phase angles of $0^{\circ}, 22.5^{\circ}, 45^{\circ}$, and $90^{\circ}$ (approximately $0.00,0.24,0.49,0.98$ seconds representing an offset of $0,1 / 16^{\text {th }} 1 / 8^{\text {th }}$, and $1 / 4^{\text {th }}$ the period) (Figure $2.1 C \& D$ ). Below a threshold $90^{\circ}$ we observed potentiation of the synapse as measured by a mean decrease in $\mathrm{R}_{c}$ (Figure 2.3). At 40 minutes, the $0^{\circ}, 22.5^{\circ}, 45^{\circ}$, and $90^{\circ}$ degree phase angles have not deviated significantly from baseline at a threshold level of $p<0.05\left(0^{\circ}\right.$ $\Delta \mathrm{gj}^{-1}$ at $40, \mathrm{df}=4, \mathrm{~V}=1, \mathrm{p}=0.125$, Wilcoxon rank sum $),\left(22.5^{\circ} \Delta \mathrm{gj}^{-1}\right.$ at $40, \mathrm{df}=4, \mathrm{~V}=0, \mathrm{p}$ $=0.0625$, Wilcoxon rank sum), $\left(45^{\circ} \Delta g_{j}^{-1}\right.$ at $40, \mathrm{df}=4, \mathrm{~V}=2, \mathrm{p}=0.1875$, Wilcoxon rank sum), (90 $\Delta \mathrm{g}^{-1}$ at $40, \mathrm{df}=4, \mathrm{~V}=15, \mathrm{p}=0.0625$, Wilcoxon rank sum). This does not to suggest that the change between groups is equivalent at 40 minutes. Depression at $90^{\circ}$ and potentiation at smaller phase angles resulted in a significant change of coupling between groups at 40 minutes $\left(\Delta \mathrm{g}^{-1}\right.$ by Condition, $\mathrm{df}=3$, chi-squared $=8.897, \mathrm{p}=$ 0.031, Kruskal-Wallis). This appears as the Time:Condition effect in in the linear model for coupling resistance $\left(g_{j}{ }^{-1}\right.$ Time:Condition $=F(3,59)=1.964, p=0.129, p_{\text {adj }}=0.219$, Linear Mixed-Effects) $\left(R_{c}\right.$ Time:Condition $=F(3,63)=3.28, p=0.027, p_{a d j}=0.053$, 
Linear Mixed-Effects). Resistance increased over time with a $90^{\circ}$ protocol by 0.002 to $0.007 \mathrm{M} \Omega / 20$ Minutes (as estimated from the $R_{c}$ and $g_{j}^{-1}$ measurements respectively) relative to control. All other groups decreased relative to control. Coupling coefficient was altered as well $\left(C C\right.$ Time:Condition $=F(3,154)=3.246, p=0.024, p_{\text {adj }}=0.053$, Linear Mixed-Effects) decreasing by $-0.002\left(\Delta \mathrm{mV}_{2} / \Delta \mathrm{mV}_{1}\right) / 20$ Minutes in the $90^{\circ}$ group and increasing by 0.001 in the $22.5^{\circ}$ and $45^{\circ}$ groups. The full results from the linear model are provided in Table 2.3.

Taking the above experiments together, the results are consistent with a lack of synchrony being responsible for altering coupling resistance (See Figures 2.2A \& 2.3). However, it appears that phase alone is insufficient to fully predict this, as Depolarized Async and Naturalistic $90^{\circ}$ possess similar delays and periods with only depolarization differing. Despite having the same phase angle, at different levels of depolarizations the sign of gap junction regulation is flipped. This observation supports a role for another electrophysiological factor, such as depolarization, to interact with synchrony in influencing coupling.

Phase-shifted endogenous waveforms do not consistently alter coupling Model comparison does not reveal a single better predictor than phase

Given that baseline LC activity differs across animals, we sought to represent this variability and determine potential electrophysiological factors other than phase that might be more predictive of altered coupling. We repeated the previous experiment, except instead of using a single naturalistic waveform across preparations we used each network's own baseline activity from which to derive the stimulus waveform ("Individualized Stimulus"). We performed these experiments with one additional group with a phase angle of $180^{\circ}(1 / 2$ the period) (Figure 2.1C \& D). Given cells' diversity of not 
only excitability but also period, a range of delays (in seconds) is represented in each phase angle group.

The "Individualized" protocols altered coupling throughout the experimental groups, but delay had a markedly less consistent effect among these heterogeneous waveforms (Figure 2.4A). While $R_{c}$ appears to have decreased in some preparations (Figure 2.4A), none of the treatment groups changed significantly from baseline $\left(0^{\circ} \Delta \mathrm{g}_{\mathrm{j}}^{-1}\right.$ at $40, \mathrm{df}=6, \mathrm{~V}=9, \mathrm{p}=0.469$, Wilcoxon rank sum $),\left(22.5^{\circ} \Delta \mathrm{g}^{-1}\right.$ at $40, \mathrm{df}=3, \mathrm{~V}=0, \mathrm{p}=0$

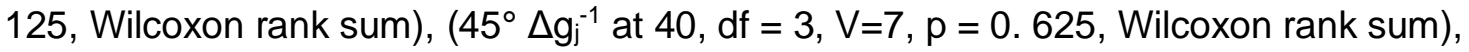
$\left(90^{\circ} \Delta \mathrm{g}^{-1}\right.$ at $40, \mathrm{df}=2, \mathrm{~V}=2, \mathrm{p}=0.75$, Wilcoxon rank sum $),\left(180^{\circ} \Delta \mathrm{g}_{\mathrm{j}}^{-1}\right.$ at $40, \mathrm{df}=4, \mathrm{~V}=4$, $p=0.438$, Wilcoxon rank sum). Additionally, unlike the standardized protocols above, using individualized protocols resulted in groups that were not separable at 40 minutes by change in coupling $\left(\Delta R_{c}\right.$ by Condition, $d f=4$, chi-squared $=2.664, p=0.616$, Kruskal-Wallis). The interaction effect with the smallest $p$ value was input resistance $\left(R_{11}\right.$ Time:Condition $=F(4,106)=2.312, p=0.062, p_{a d j}=0.107$, Linear Mixed-Effects $)$. Coupling resistance $\left(R_{c}\right.$ Time:Condition $=F(4,64)=1.942, p=0.114, p_{a d j}=0.183$, Linear Mixed-Effects) (Figure 2.4A) and coupling coefficient (CC Time:Condition = F $(4,154)=1.115, p=0.352, p_{\text {adj }}=0.444$, Linear Mixed-Effects $)$ were likewise inconsistently affected. A full list of the tested variables and the resultant $p$ values is provided in Table 2.4 .

The Individualized LC recordings used in these experiments represented substantial variability among stimuli, as control activity among animals is not uniform. This enabled us to explore if other electrophysiological features were predictive of changes in coupling, as might be expected based on previous studies (Lane et al., 2016; Fricker et al., 2020). To this end, we tested 15 variables representing depolarization features (On duration, Duty cycle, Area under the curve (AUC), Normalized AUC 
(AUC/period), Max mV, Median mV, Minimum mV), and agreement between depolarization and stimuli timing in both cells (Phase, Phase as discrete conditions, Delay, Percent delay, Stimulus Correlation, Joint AUC (AUC of minimum voltage in either cell at any given time), Normalized joint AUC (Joint AUC / Period)). We ranked the efficacy of models of these variables using the difference in Adjusted Akaike information criterion (AICc) of each model independently (Figure 2.4B). AICc provides a measure of the predictive power of a model corrected for the number of terms where smaller AICc values indicate a better model. While none of the waveform-derived measures outperformed phase as a predictor, given the insufficiency of phase alone to capture the results of the previous we cannot rule that other factors, or interactions of factors such as those listed above, may be involved in modulating coupling induced by phase differences.

Dynamic Clamp reveals a detrimental effect of coupling strength and delay on cells' target output

The goals of this experiment were twofold. First, to determine the effects of coupling strength on synchrony between two cells with a continuous range of delay times in their relative activities by comparing the activity of two artificially coupled cells in two different ganglia across a range of coupling conductances. Second, to investigate how coupling and delay interact to "pull" the cell out of its normal pattern of activity by comparing experimentally varied coupling strength and delay on a cell's activity relative to its own baseline activity (i.e. without artificial coupling). Since the previous analysis provided no predictor for change in coupling that was clearly superior to phase/synchrony, we continued using loss of synchrony between cells to test for a delay dependent "cost" to the cell that is minimized by electrical synapse potentiation or depression. If such a cost existed, we would predict it manifests at a threshold less than 
or equal to a delay of 0.98 seconds $\left(90^{\circ}\right.$ for the Naturalistic waveform) given that depression is seen at this delay (Figure 2.3). However, given the variable responses seen in our endogenous waveform experiments (Figure 2.4) we cannot rule out that that this threshold would differ between cells, and certainly the sensitivity to delay will be influenced by the magnitude of artificial coupling.

In order to use a truly naturalistic voltage stimulus we utilized dynamic clamp to take advantage of endogenous cell activity in a manner that provides a near continuous range of delays and complete control over coupling properties. To do so, we introduced a simulated gap junction between two LCs in separate, active CGs (Figure 2.5A) and recorded LC activity from both networks under six artificial synaptic strengths $(0,0.025$, $0.05,0.1,0.15$, and $0.2 \mu \mathrm{S})$. These recordings were sufficiently long (115-300 seconds) to gain a roughly continuous sampling of delays between the preparations (Figure 2.5B). To visualize the deviation from control activity, we plotted the median correlation coefficient (adapted from methods previously reported (Lane et al., 2016)) between a burst and all control bursts, with respect to coupling conductance and delay (Figure $2.5 \mathrm{C})$. With these data we examined how well artificially coupling cells can promote more synchronous activity between cells across networks. We were also able to compare a cell's activity to its control activity to assess how delay and coupling alter cell output from baseline.

We performed this experiment with one pair of CGs with naturally similar LC depolarization amplitudes and one pair of networks consisting of LCs with naturally varying depolarization amplitudes. To assess the difference between bursts of a given condition and baseline activity, we correlated all overlapping bursts of a given LC against all those of its own control activity (i.e. with no artificial synapse present). Summarizing 
the resultant distribution provided a measure of similarity between a cell's control activity and that of a given delay and coupling strength.

We find that in both trials, correlation coefficient between the networks (i.e. similarity to each other not to a control output) was maximized by a high coupling conductance and low delay (Figure 2.6A, $A v B$ ). This was expected and serves to confirm the system was operating correctly. We found in both trials that the self-similarity (i.e. correlation against a cell's baseline activity) decreased with increased coupling conductance and that this effect appears more severe as the delay between networks increases (Figure 2.6A, $A v A^{\prime}$ and $B v B^{\prime} ; 6 \mathrm{~B}$ ). In other words, the stronger the synapse between two cells and the longer the delay, the less similar a given cell will produce output resembling its own baseline activity (measured as a correlation coefficient). While output correlation was affected by coupling conductance in a roughly linear manner, delay affected correlation in an approximately parabolic manner (Figure 2.6A). This relationship was shifted relative to zero delay in the experiment with mismatched burst amplitudes and durations (Preparation 2) and inverted by switching the reference cell, thereby turning a negative delay positive (Figure 2.6A), contrast references within a trial). Finally, we observed that when cells are mismatched in excitability, there are pronounced differential sensitives between cells (Figure 2.6A; 2.6B, Preparation 2).

\section{Discussion}

The goals of this study were twofold. First, we set out to determine whether plasticity of electrical synapses can be induced solely by activity-dependent means (e.g. a transjunctional voltage), and second to shed light on the functional importance of coupling modulation in highly synchronous networks. This required a model system in which one can observe and maintain natural synchronous activity among constituent neurons and completely control neuron activity with voltage-clamp. With the crustacean 
cardiac ganglion, we achieved the experimental control necessary to decouple activity from other network parameters to demonstrate a purely activity-dependent modulation of coupling that is directionally dependent on the degree of difference in activity between the coupled cells. We then performed artificial coupling experiments to explore how eLTP can help restore synchrony in a presumably compensatory fashion. However, we also show that after a point, increased coupling alone fails to prevent divergence from synchrony, at which we suggest it becomes advantageous for networks to "cut off" the aberrant cell through eLTD, salvaging the output of the remainder of the network.

Loss of synchrony, and not increased depolarization, induces eLTP in LCs

Our previous work demonstrated eLTP in crustacean cardiac ganglia, resulting from exposure of LCs to TEA, causing not only hyperexcitability and increased depolarization (Ransdell et al., 2012b, 2013a) but also loss of synchrony among LCs (Lane et al., 2016). These experiments did not determine whether the salient signaling inducing eLTP was increased activity of the TEA-exposed LCs or the loss of coordinated activity (or both). In this study, we find increased depolarization is insufficient to alter $R_{c}$ (Figure 2.2). Instead, a delay between depolarizations is required. This is repeated in three different experiments (Figures 2.2-2.4), with protocols that vary in the level of depolarization, and the phase delay between cells. Phase delay can elicit coupling modulation distinct and is abolished by calcium channel blockade (Figure 2.2B), which agrees with previously reported activity dependent gap junction plasticity (Welzel and Schuster, 2018; Fricker et al., 2020). These experiments are consistent with the hypothesis of a voltage delay induced, calcium-mediated mechanism of electrical synapse potentiation (Haas et al., 2016). However, we do not yet know whether a meaningful differential in calcium forms across the synapse, as seen when one coupled 
cell is driven in the TRN (Fricker et al., 2020), acting as the salient signal, or if this effect is driven by a different signal.

The magnitude of phase difference may determine whether eLTP or eLTD is induced

Here, for the first time to our knowledge, we find the same depolarizing stimulus produces both eLTP and eLTD contingent upon the delay between cells. This appears as a phase by time effect in our model; seen clearly in Figure 2.3. We observed clear and widespread eLTD with the Naturalistic stimulus at a phase angle of $90^{\circ}$ and less consistently in the Individualized protocols as well. It appears that the threshold dividing eLTP and eLTD may be smaller than $90^{\circ}$ as some preparations at $45^{\circ}$ also displayed eLTD. Furthermore, we are not ruling out a potential interaction with depolarization. For example, the stimulus protocols seen in Depolarized Async (Figure 2.2B) and Naturalistic $90^{\circ}$ (Figure 2.3) have similar timings, with virtually no overlap in the activation of the two cells but differ substantially in their depolarization amplitude and direction of modulation. Indeed, in general our most robust eLTP effects were elicited by our protocols that depolarize more (see traces in Figure 2.1C and effects shown in Figure 2.2 to Figures 2.3 and 2.4). Therefore, the "rules" by which a given activity pattern elicits eLTP or eLTD may vary across networks due to variability in the underlying rhythms across individuals (Marder, 2011; Williams et al., 2013) and this suggests that more factors are at work. While other electrophysiological features may influence coupling, in our results we find none more predictive than phase (Figure 2.4B). However, it seems likely based on our data that membrane depolarization (or lack thereof) may play a meaningful role in determining synaptic plasticity in this system. A recent study from Fricker et al. is consistent with this hypothesis. They report when stimulation of TRN neurons evoked spiking that eLTP is induced, whereas evoked bursting and 
subsequent higher intracellular calcium results in eLTD (Fricker et al., 2020). Our work suggests regulation may involve the sustained existence of a calcium gradient, but additional studies will be necessary to indicate the precise intracellular triggers of eLTP and eLTD.

\section{Coupling conductance increases sensitivity of cell output to}

\section{phase delay}

Excitatory neuromodulators (Cruz-Bermudez and Marder, 2007; Lane et al., 2018) or ionic current blockers (Ransdell et al., 2013a; Lane et al., 2016) suppresses coordination between neurons. This loss of synchrony is attenuated (Lane et al 2016) or prevented (Lane et al., 2018) with an associated increased in coupling. This does not explain the utility of a biphasic response where $R_{C}$ is decreased at small delays and increased at larger ones (Figure 2.3). Utilizing hybrid networks formed between LCs of two different ganglia with an artificial electrical synapse, we tested for changes in cell output as an effect of delay between neurons, and coupling conductance (Figure 2.5). The logic of the experiment is as follows. First by comparing the activity of two artificially coupled cells in two different ganglia across a range of coupling conductances, we can determine how well coupling is able to promote more synchronous activity between (naturally variable) cells. We predicted that with smaller delays between the onset of activity of these two cells, increased coupling would increase their synchrony. However, at larger delays, not only would strong coupling likely be ineffective at synchronizing cell activity, but may also produce aberrant cell activity (relative to the cells' "home"

networks). This leads to the second aspect of the logic of this experiment: by comparing the effects of experimentally varied coupling and delay on a cell's activity relative to its 
own baseline activity (i.e. at $0 \mu \mathrm{S}$ ), we demonstrate how coupling and delay may interact to "pull" the cell out of its normal pattern of output. We predicted that at some level of delay, it would become advantageous to reduce coupling, minimizing disruption of each cell's natural activity.

These inter-related predictions were supported by the data (Figure 2.6): the waveforms of artificially coupled cells are most similar under conditions of high coupling conductance and low delay in activity between the cells. Conversely, activity across networks is most dissimilar when cells are subject to low conductance and high activity delay. Additionally, when we look at these data from the perspective of each cell's own native biological network, we indeed find that a cell's output is deformed from control with increased coupling in a delay-dependent manner where large delays cause the largest deviation (Figure 2.6). This occurs even at coupling levels typically found in populations of these networks. Conversely, when coupling is reduced to lower than typical biological levels (i.e. eLTD), this exerts a protective effect whereby a cell is no longer pulled from its typical pattern of activity by an artificially coupled partner with a high amount of delay.

Taken together these experiments support the idea of a nonlinear cost to a network's output, whereby a cell with disrupted activity within a network may be "pulled back in" to normal synchrony through electrical synaptic potentiation, but a severely impaired cell can be "cut off" from the remaining healthy cells via synaptic depression if its activity onset differs too greatly. How this "decision" to rescue or abandon an improperly behaving cell is determined remains a compelling question for future experimentation, both computational and physiological.

\section{Conclusion}


Electrical synapse plasticity is present across taxa in both innexin and connexin based synaptic coupling (Haas et al., 2011c; Lane et al., 2016; Welzel and Schuster, 2018). Despite the multiple means documented by which circuits can tune their electrical synapses, our data are the first to demonstrate that timing of voltage activity can dynamically modulate electrical synapses. Further, with our study there is now combined evidence that this too is a calcium-mediated (Sevetson 2017) and likely depolarizationdependent mechanism (Fricker et al., 2020) that is conserved across invertebrates and vertebrates. We suspect that the features which tune these properties will underly the dynamics of long-term electrical synapse plasticity akin to what has been proposed for membrane excitability and demonstrated in silico (O'Leary et al., 2014a). These mechanisms undoubtedly work in conjunction with other regulatory mechanisms of cellular and synaptic properties to provide robust constraints on network output. 
Figure Legends, Tables, Extended Data, and Multimedia

Figure 2.1 Synchronous, normative large cell (LC) depolarizations can be manipulated through network silencing followed by voltage clamp.

A

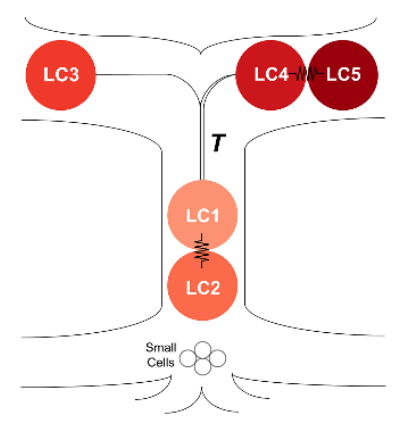

B

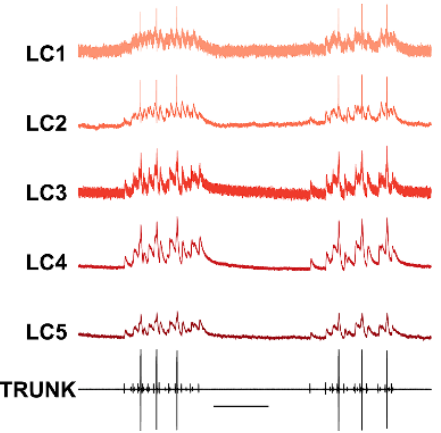

C

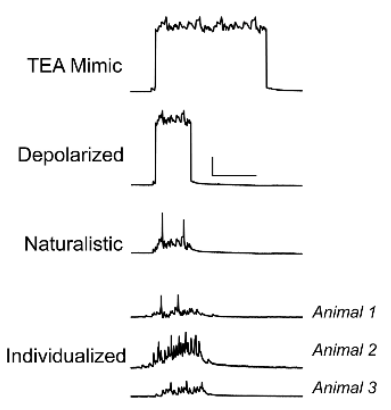

D

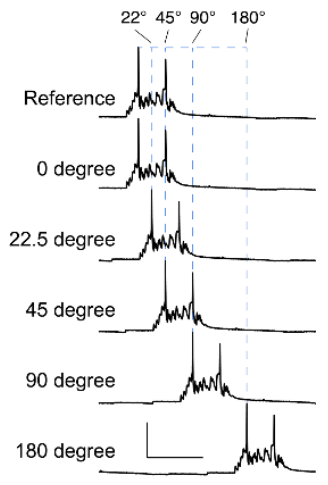

A) The five motor neurons (Large Cells, LCs) of the cardiac ganglion are driven by four pacemaker interneurons (Small Cells) through chemical and electrical synaptic excitation. B) LCs depolarize synchronously, as shown by simultaneous intracellular recordings. A simultaneous extracellular recording made from the nerve Trunk (marked with a "T" in Panel 1A) detects 3 LC action potentials per burst (large events) as well as the pacemaker activity of the Small Cells (small events). Time scale bar is 1 second. C) After Small Cells are silenced by displacing physiological saline with isotonic sucrose fictive LC activity patterns can be induced with two electrode voltage clamp. Fictive activity patterns are designed to mimic i) the effects of TEA (“TEA Mimic"), ii) aberrantly high depolarization without increased length of the depolarization (“Depolarized”), iii) normative activity using a single standardized depolarization ("Naturalistic"), and iv) normative activity using the cell's own activity (“Individualized”, examples shown from three different animals). Bars represent $10 \mathrm{mV}$ and 1 Second. D) Experimentally controlled shifts from synchronous activity across two LCs are expressed as phase angles where an offset of $1 / 4$ the period is $90^{\circ}$. Protocols with delays between LCs 
ranging from 0 to $1 / 4$ of the period were created with the Naturalistic stimulus and from 0 to $1 / 2$ the period for the Individualized stimuli. 
Figure 2.2 Electrical synapse potentiation (eLTP) requires desynchronized activity and $\mathrm{Ca}^{2+}$, not depolarization alone
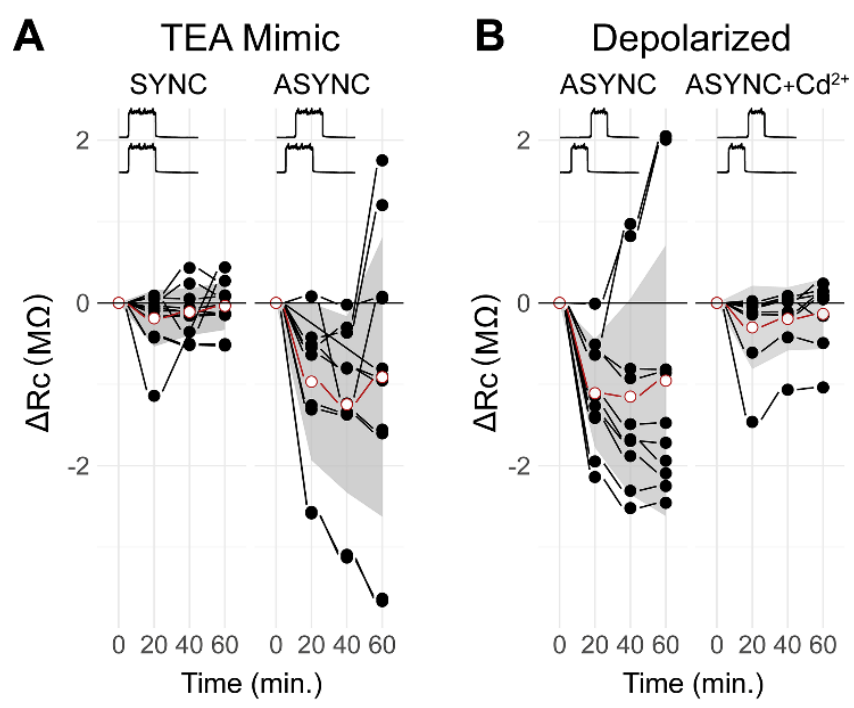

A) TEA mimetic stimuli (increased depolarization and duration) applied synchronously (SYNC) in coupled LCs result in little to no eLTP (i.e. a decrease in coupling resistance, $\mathrm{R}_{\mathrm{c}}$ ). Asynchronous depolarization across gap junctions (ASYNC) results in a substantial eLTP within 20 minutes. Filled circles connected by lines show change of $R_{c}$ from baseline for a given cell over time. All cells are shown individually, but two cells from the same network (i.e. that share a gap junction) are treated as pseudo-replicates in the analyses. Open circles track the mean, gray bands show standard deviation. B) Decreasing the duration of the depolarization relative to the TEA Mimic stimulus (Depolarized ASYNC) does not abolish the eLTP effect. However, potentiation does not occur with the same stimulus protocol in the presence of $\mathrm{Cd}^{2+}$ (Depolarized ASYNC+$+\mathrm{Cd}^{2+}$ ) suggesting that calcium influx is required as well. 
Figure 2.3 Desynchronization with a naturalistic stimulus below a $90^{\circ}$ phase shift depresses the LC 4/5 electrical synapse, smaller delays potentiate the synapse.

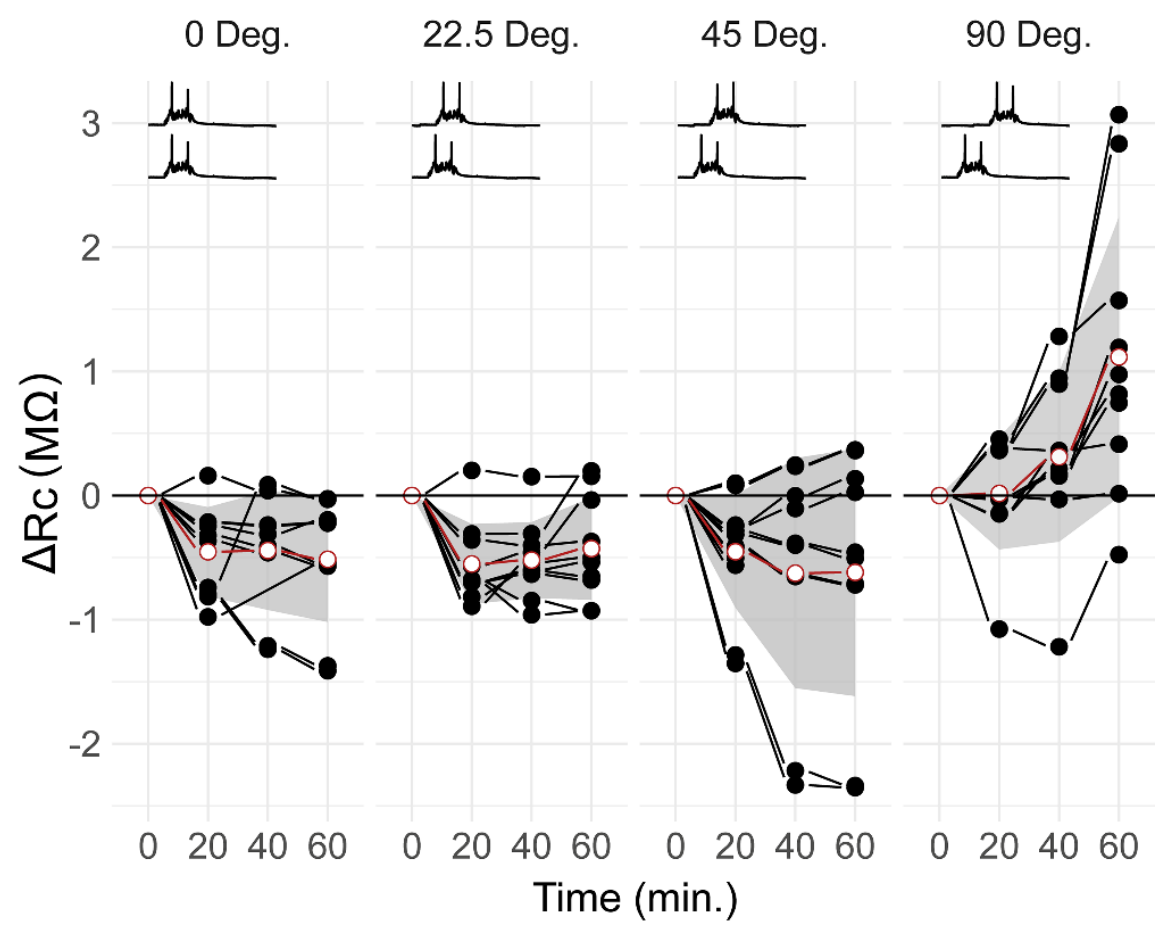

We used the Naturalistic waveform to examine the effects of asynchronous stimulation at more normative voltages. At these more normative voltages, the sign of change (i.e. eLTP or eLTD) appears dependent on the magnitude of delay. A naturalistic waveform applied with a delay less than $90^{\circ}$ (i.e. an offset of $1 / 4^{\text {th }}$ the period between cells) results in eLTP, while a delay of $90^{\circ}$ results in eLTP. Filled circles connected by lines show change of $R_{c}$ from baseline for a given cell over time. All cells are shown individually, but two cells from the same network (i.e. that share a gap junction) are treated as pseudoreplicates in the analyses. Open circles track the mean, gray bands standard deviation. 
Figure 2.4 Phase outperforms waveform derived metrics in explaining changes in coupling.

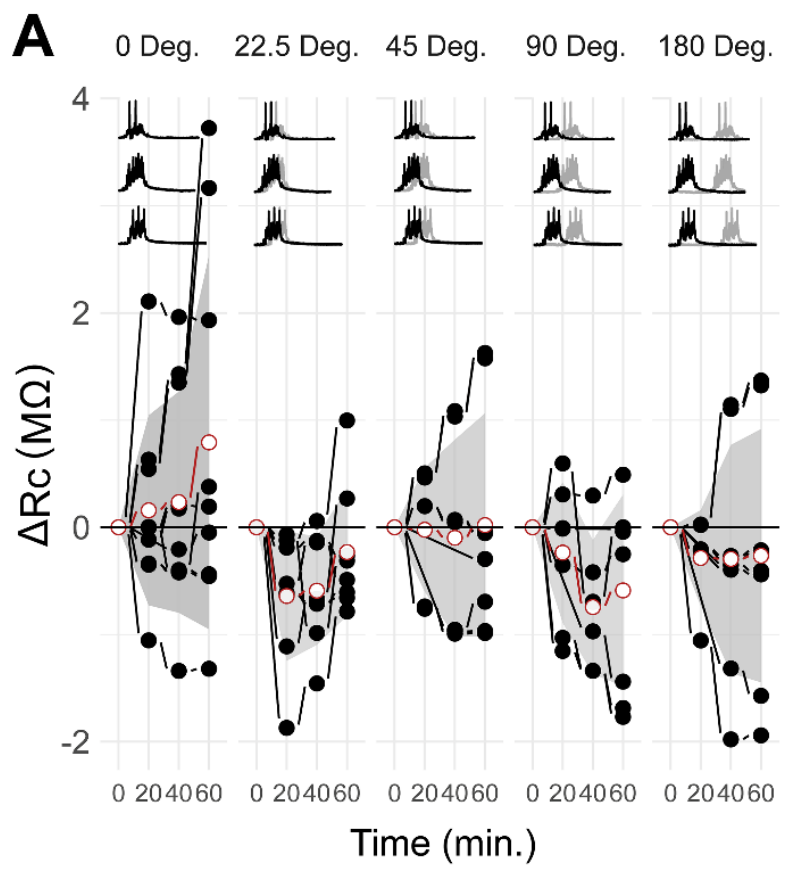

B

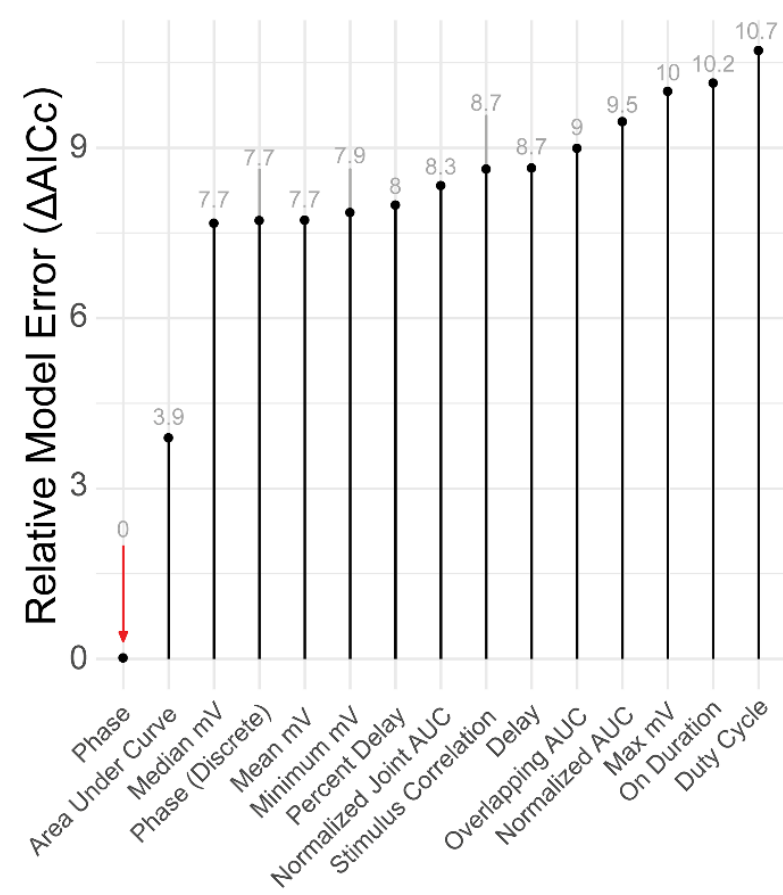

A) Using a network's endogenous activity at delays up to $180^{\circ}$ (an offset of $1 / 2$ the period) fails to cleanly reproduce the effect seen with stricter experimental control. As before, black and red dots represent the change in individual cells and the mean respectively whereas the gray band represents the standard deviation. Use of individualized stimuli cause more varied effects on eLTP and eLTD than under any of the standardized treatments (TEA Mimetic, Depolarized, or Naturalistic). However, overall eLTP is induced at $22.5^{\circ}$, consistent with the other stimulus protocols. B) We tested the efficacy of other predictors derived from the individualized waveforms (e.g. total depolarization) to explain the change in coupling resistance. To evaluate models, we used Akaike information criterion corrected (AICC) for the number of terms in the model and plot the difference from the model with the lowest AICc $(\triangle \mathrm{AICc})$. Because the period of 
individualized stimuli is variable, we represented phase as a continuous variable. Phase outperformed all other tested metrics. 
Figure 2.5 LC output is altered by phase delay in a coupling conductance dependent manner.

$\boldsymbol{A}$

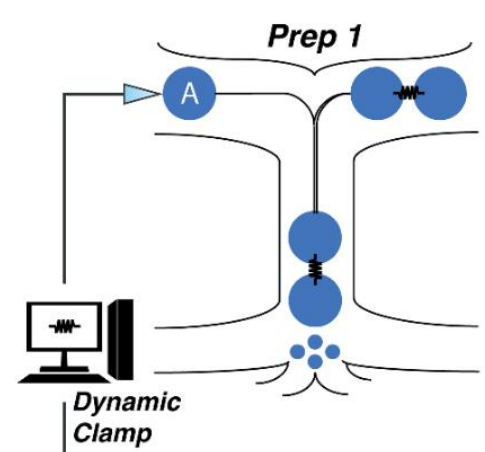

Clamp

B

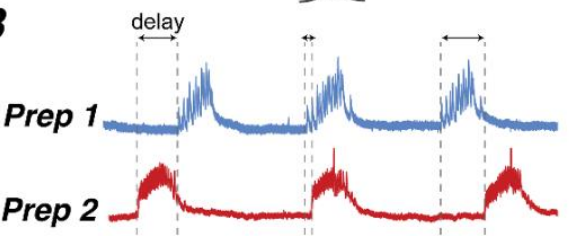

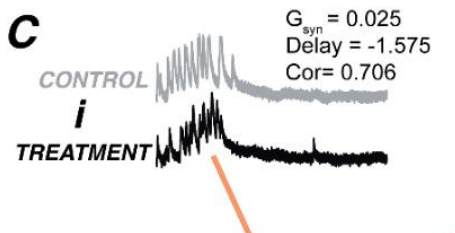

$\mathrm{G}_{\mathrm{syn}}=0.025$

Delay $=0.876$

Cor $=0.753$

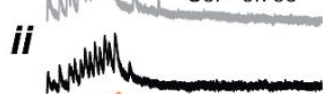

.5

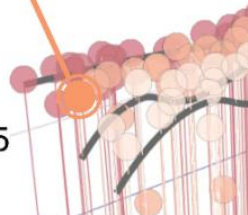

๖ั

$-0.5$

$$
\begin{aligned}
& \mathrm{G}_{\text {syn }}=0.2 \\
& \text { Delay }=-0.81
\end{aligned}
$$$$
\text { Cor }=-0.17
$$

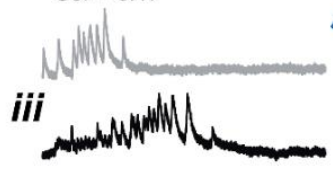

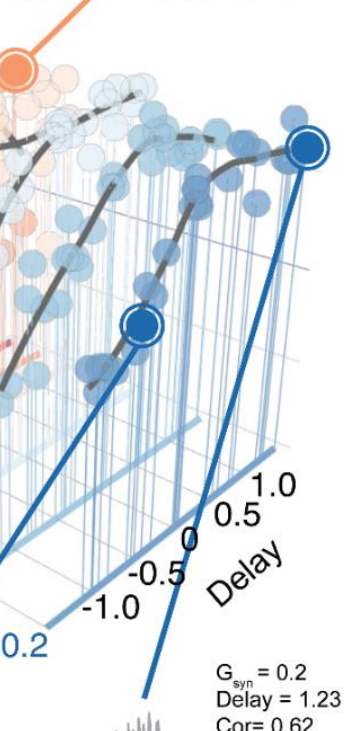

Cor $=0.62$

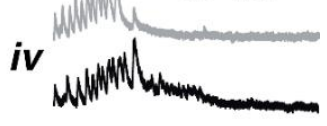

A) Using dynamic clamp, we artificially coupled two LC motor neurons from two different networks (i.e. animals) with their distinct ongoing rhythms intact. The simulated reciprocal synapse had a strength between 0 and 0.2 uS. B) As a result of the different cycle periods of endogenous activity between the networks, the artificially coupled LCs experienced activity with a continuous range of positive and negative delays relative to one another. The start and end of each cell's bursts was defined based on spiking detected in the extracellular recordings (see Figure 2.1B, Trunk). C) To measure similarity to each cell's baseline activity, each burst is correlated with every burst of its network's control activity. The median value of this set (Cor) represents the similarity or synchrony between cells, and is then plotted for a given artificial synapse strength $\left(G_{\text {syn }}\right)$ 
and phase delay (Delay). Four example traces of a control (gray) and treatment (black) burst are shown accompanied by the coupling strength, delay, and similarity (correlation) to control activity. Highlighted comparisons show i) low coupling, high negative delay, ii) low coupling, high positive delay, iii) high coupling, negative delay, and iv) high coupling high positive delay. In this way, we can represent how similar (high value of Cor) or dissimilar (low values of Cor) the artificially coupled cells are to one another as a function of the interaction between synapse strength $\left(\mathrm{G}_{\text {syn }}\right)$ and the level of synchrony, or phase delay (Delay). 
Figure 2.6 LC output is impaired by delay in a coupling conductance

\section{dependent manner}

A
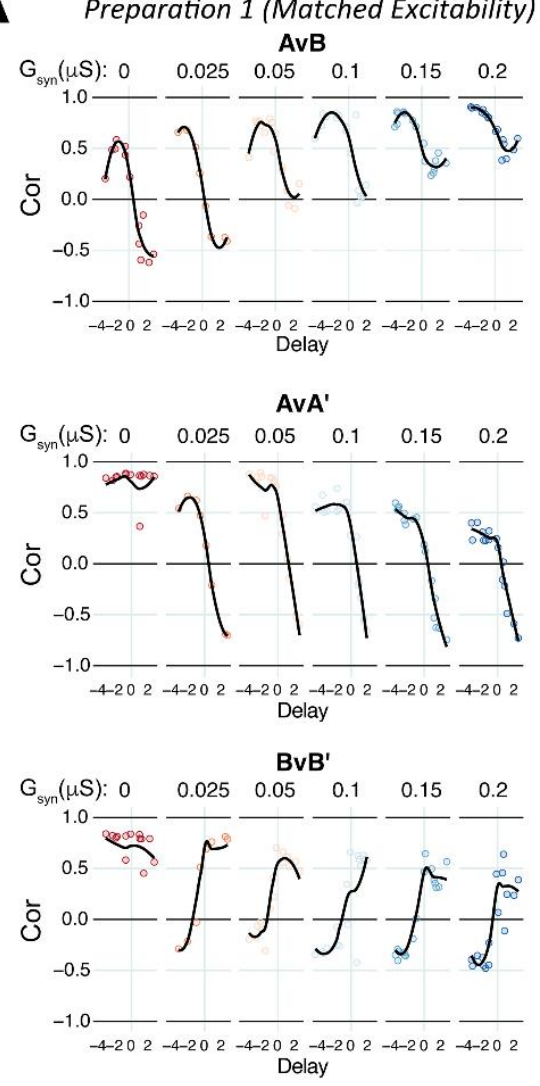

B

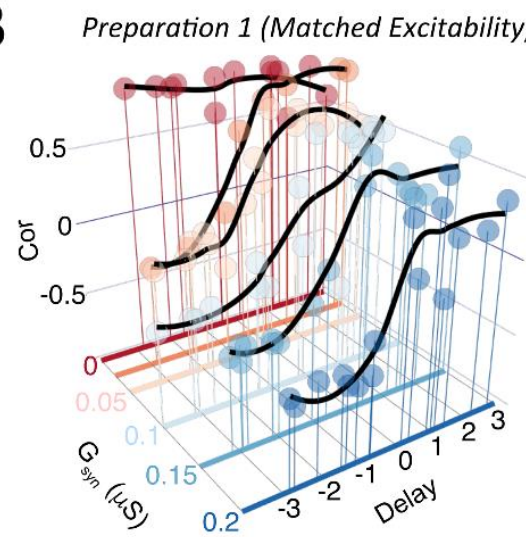

Preparation 2 (Mismatched Excitability)
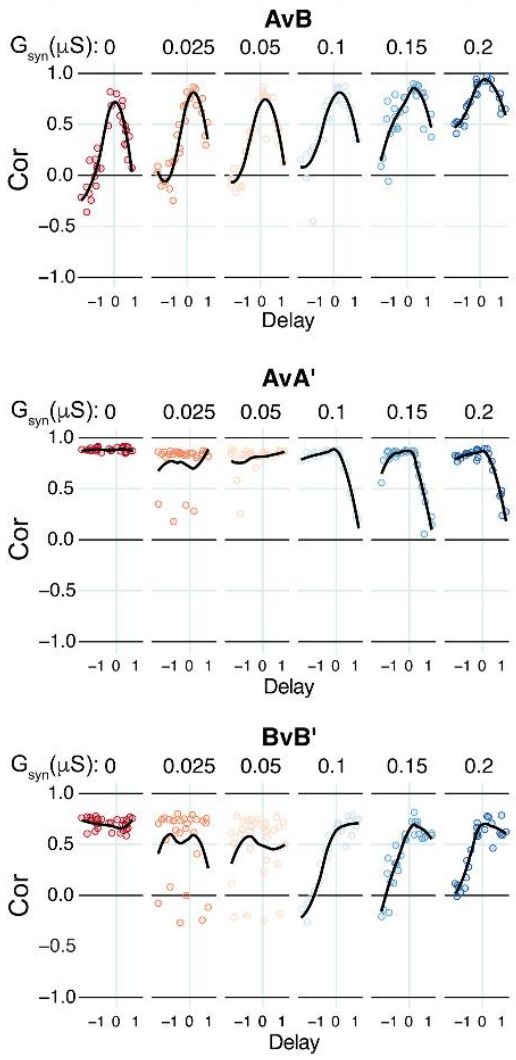

Preparation 2 (Mismatched Excitability)

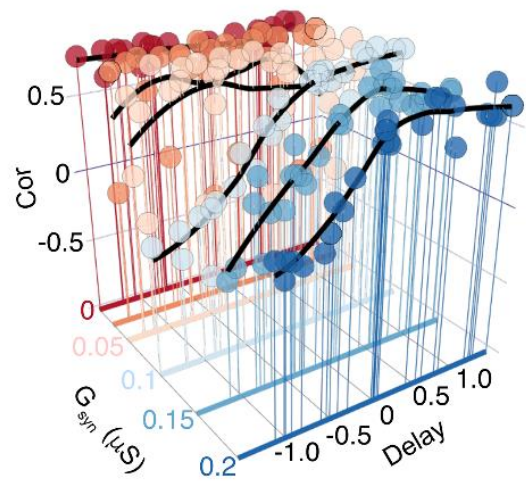

A) $A v B$ : Correlating burst activity (Cor, or the similarity between two cells) between preparations (ganglion A vs ganglion B) shows that both delay between burst onset and coupling strength ( $\mathrm{G}_{\mathrm{syn}}$ from $0-0.2 \mathrm{mS}$, noted above each panel) alter burst similarity. 
Hybrid networks of ganglia with similar excitability (Preparation 1) and dissimilar excitability (Preparation 2), display the same trend with different sensitivities to delay and coupling strength. In both cases, coupling increases overall similarity with delay altering similarity non-linearity. The maxima and sensitivity to delay appears to differ across cell pairs. AvA' and BvB': To determine whether coupling strength and delay interact in a manner that causes one of the artificially coupled cells to change its activity relative to its endogenous network, we plotted the similarity scores (refer to figure 2.5) of each cell to its own baseline output (A' or B' respectively) as a function of $\mathrm{G}_{\mathrm{syn}}$ and delay. Individual cells are differentially sensitive to their partner's activity, and the hybrid network in Trial 1 appears more sensitive than that of Trial 2 . When $G_{\text {syn }}$ is 0 (no artificial synapse), waveform output for a given cell is consistent over time. As synapse strength is increased, each cell is more and more "pulled" out of its endogenous waveform as a function of both the delay between cells and the strength of the synapse. Delays closer to 0 result in less loss of similarity to baseline activity. Each cell in a hybrid network shows the opposite pattern to its partner (e.g. decreasing at positive delays vs decreasing at negative delays - contrast $A v A^{\prime}$ and $\left.B v B^{\prime}\right)$, as a positive delay from the vantage point of one network will be a negative delay from the other and because bursts are not symmetrical. Similarity to control activity is measured by the median of the population correlation coefficients between a single burst and a set of control bursts. To aid in visualizing trends over delay, these medians have been fit with a LOESS regression. B) For better visualization, the same data shown in BvB' are plotted in 3 dimensions with respect to both $G_{\text {syn }}$ and Delay. The minimum $G_{\text {syn }}$ at which Delay compromises similarity differs between the two preparations. Despite the different excitabilities of the composite networks and differing sensitivity to $G_{\text {syn }}$ and Delay, the overall shape of the relationship is remarkably similar. 
Table 2.1: TEA Mimic Sync \& TEA Mimic Async.

\begin{tabular}{|c|c|c|c|c|c|c|c|c|}
\hline $\begin{array}{c}\text { Dependent } \\
\text { Variable }\end{array}$ & Parameter & df & F Value & p-Value & FDR Adjusted $p$ & Parameter & Effect Estimate & \\
\hline \multirow[t]{4}{*}{ CC } & (Intercept) & $(1,84)$ & 345.491 & 0 & 0 & & & \\
\hline & Time & $(1,84)$ & 2.943 & 0.09 & 0.126 & Time Effect & 0 & $\Delta \mathrm{mV} / \Delta \mathrm{mV}$ \\
\hline & Condition & $(1,11)$ & 12.647 & 0.005 & 0.014 & $\begin{array}{l}\text { TEA Mimic Async } \\
\text { Effect }\end{array}$ & -0.274 & $\Delta \mathrm{mV} / \Delta \mathrm{mV}$ \\
\hline & Time:Condition & $(1,84)$ & 9.027 & 0.004 & 0.012 & Interaction Effect & 0.002 & $(\Delta \mathrm{mV} / \Delta \mathrm{mV}) / 20 \mathrm{Min}$ \\
\hline \multirow[t]{4}{*}{ gj-1 } & (Intercept) & $(1,32)$ & 40.984 & 0 & 0 & & & \\
\hline & Time & $(1,32)$ & 4.484 & 0.042 & 0.085 & Time Effect & 0 & $\mathrm{M} \Omega$ \\
\hline & Condition & $(1,11)$ & 5.096 & 0.045 & 0.085 & $\begin{array}{l}\text { TEA Mimic Async } \\
\text { Effect }\end{array}$ & 3.172 & $\mathrm{M} \Omega$ \\
\hline & Time:Condition & $(1,32)$ & 4.781 & 0.036 & 0.084 & Interaction Effect & -0.016 & $\mathrm{M} \Omega / 20 \mathrm{Min}$ \\
\hline \multirow[t]{4}{*}{ R1 } & (Intercept) & $(1,44)$ & 195.587 & 0 & 0 & & & \\
\hline & Time & $(1,44)$ & 0.04 & 0.842 & 0.873 & Time Effect & -0.01 & $\mathrm{M} \Omega$ \\
\hline & Condition & $(1,11)$ & 0.052 & 0.824 & 0.873 & $\begin{array}{l}\text { TEA Mimic Async } \\
\text { Effect }\end{array}$ & -0.57 & $\mathrm{M} \Omega$ \\
\hline & Time:Condition & $(1,44)$ & 5.681 & 0.022 & 0.058 & Interaction Effect & 0.023 & $\mathrm{M} \Omega / 20 \mathrm{Min}$ \\
\hline \multirow[t]{4}{*}{ R11 } & (Intercept) & $(1,63)$ & 119.355 & 0 & 0 & & & \\
\hline & Time & $(1,63)$ & 0.228 & 0.635 & 0.711 & Time Effect & -0.002 & $\mathrm{M} \Omega$ \\
\hline & Condition & $(1,11)$ & 0.001 & 0.974 & 0.974 & $\begin{array}{l}\text { TEA Mimic Async } \\
\text { Effect }\end{array}$ & -0.204 & $\mathrm{M} \Omega$ \\
\hline & Time:Condition & $(1,63)$ & 3.972 & 0.051 & 0.089 & Interaction Effect & 0.006 & $M \Omega / 20$ Min. \\
\hline \multirow[t]{4}{*}{ R12 } & (Intercept) & $(1,36)$ & 74.494 & 0 & 0 & & & \\
\hline & Time & $(1,36)$ & 1.138 & 0.293 & 0.348 & Time Effect & -0.001 & $\mathrm{M} \Omega$ \\
\hline & Condition & $(1,11)$ & 2.458 & 0.145 & 0.185 & $\begin{array}{l}\text { TEA Mimic Async } \\
\text { Effect }\end{array}$ & -0.656 & $\mathrm{M} \Omega$ \\
\hline & Time:Condition & $(1,36)$ & 2.76 & 0.105 & 0.14 & Interaction Effect & 0.007 & $\mathrm{M} \Omega / 20 \mathrm{Min}$. \\
\hline \multirow[t]{3}{*}{ Rc } & (Intercept) & $(1,33)$ & 45.489 & 0 & 0 & & & \\
\hline & Time & $(1,33)$ & 3.665 & 0.064 & 0.095 & Time Effect & 0 & $\mathrm{M} \Omega$ \\
\hline & Condition & $(1,11)$ & 5.275 & 0.042 & 0.085 & $\begin{array}{l}\text { TEA Mimic Async } \\
\text { Effect }\end{array}$ & 3.073 & $\mathrm{M} \Omega$ \\
\hline
\end{tabular}




\begin{tabular}{|c|c|c|c|c|c|c|c|c|}
\hline & Time:Condition & $(1,33)$ & 3.809 & 0.06 & 0.095 & Interaction Effect & -0.016 & $\mathrm{M} \Omega / 20 \mathrm{Min}$. \\
\hline \multirow[t]{3}{*}{ Vrest } & (Intercept) & $(1,39)$ & 2077.993 & 0 & 0 & & & \\
\hline & Time & $(1,39)$ & 5.626 & 0.023 & 0.058 & Time Effect & & $\mathrm{mV}$ \\
\hline & Time:Condition & $(1,39)$ & 3.658 & 0.063 & 0.095 & Interaction Effect & & $\mathrm{mV} / 20 \mathrm{Min}$. \\
\hline
\end{tabular}


Table 2.2: TEA Mimic Async \& Depolarized Async.

\begin{tabular}{|c|c|c|c|c|c|c|c|c|}
\hline $\begin{array}{c}\text { Dependent } \\
\text { Variable }\end{array}$ & Parameter & df & F Value & p-Value & $\begin{array}{l}\text { FDR Adjusted } \\
\mathrm{p}\end{array}$ & Parameter & $\begin{array}{l}\text { Effect } \\
\text { Estimate }\end{array}$ & \\
\hline \multirow[t]{4}{*}{ CC } & (Intercept) & $(1,72)$ & 414.94 & 0 & 0 & & & \\
\hline & Time & $(1,72)$ & 21.195 & 0 & 0 & Time Effect & 0.002 & $\Delta \mathrm{mV} / \Delta \mathrm{mV}$ \\
\hline & Condition & $(1,9)$ & 5.027 & 0.052 & 0.103 & $\begin{array}{l}\text { Depolarized } \\
\text { Async Effect }\end{array}$ & 0.115 & $\Delta \mathrm{mV} / \Delta \mathrm{mV}$ \\
\hline & Time:Condition & $(1,72)$ & 0.339 & 0.562 & 0.735 & Interaction Effect & 0 & $(\Delta \mathrm{mV} / \Delta \mathrm{mV}) / 20 \mathrm{Min}$ \\
\hline \multirow[t]{4}{*}{ gj-1 } & (Intercept) & $(1,29)$ & 52.432 & 0 & 0 & & & \\
\hline & Time & $(1,29)$ & 7.813 & 0.009 & 0.023 & Time Effect & -0.015 & $\mathrm{M} \Omega$ \\
\hline & Condition & $(1,9)$ & 2.829 & 0.127 & 0.209 & $\begin{array}{l}\text { Depolarized } \\
\text { Async Effect }\end{array}$ & -2.018 & $\mathrm{M} \Omega$ \\
\hline & Time:Condition & $(1,29)$ & 0.002 & 0.969 & 0.969 & Interaction Effect & 0 & $\mathrm{M} \Omega / 20 \mathrm{Min}$ \\
\hline \multirow[t]{4}{*}{ R1 } & (Intercept) & $(1,40)$ & 248.128 & 0 & 0 & & & \\
\hline & Time & $(1,40)$ & 1.772 & 0.191 & 0.297 & Time Effect & 0.013 & $\mathrm{M} \Omega$ \\
\hline & Condition & $(1,9)$ & 0.334 & 0.578 & 0.735 & $\begin{array}{l}\text { Depolarized } \\
\text { Async Effect }\end{array}$ & 0.226 & $\mathrm{M} \Omega$ \\
\hline & Time:Condition & $(1,40)$ & 3.817 & 0.058 & 0.108 & Interaction Effect & -0.016 & $\mathrm{M} \Omega / 20 \mathrm{Min}$ \\
\hline \multirow[t]{4}{*}{ R11 } & (Intercept) & $(1,55)$ & 156.42 & 0 & 0 & & & \\
\hline & Time & $(1,55)$ & 0.034 & 0.854 & 0.956 & Time Effect & 0.004 & $\mathrm{M} \Omega$ \\
\hline & Condition & $(1,9)$ & 0.53 & 0.485 & 0.679 & $\begin{array}{l}\text { Depolarized } \\
\text { Async Effect }\end{array}$ & -0.027 & $\mathrm{M} \Omega$ \\
\hline & Time:Condition & $(1,55)$ & 4.62 & 0.036 & 0.084 & Interaction Effect & -0.008 & $\mathrm{M} \Omega / 20 \mathrm{Min}$. \\
\hline R12 & (Intercept) & $(1,31)$ & 58.399 & 0 & 0 & & & \\
\hline
\end{tabular}




\begin{tabular}{|l|l|l|l|l|l|l|l|l|}
\hline & Time & $(1,31)$ & 1.283 & 0.266 & 0.392 & Time Effect & 0.006 & $\mathrm{M} \Omega$ \\
\hline & Condition & $(1,9)$ & 0.134 & 0.723 & 0.844 & $\begin{array}{l}\text { Depolarized } \\
\text { Async Effect }\end{array}$ & 0.335 & $\mathrm{M} \Omega$ \\
\hline & Time:Condition & $(1,31)$ & 4.251 & 0.048 & 0.103 & Interaction Effect & -0.008 & $\mathrm{M} \Omega / 20 \mathrm{Min}$. \\
\hline $\mathbf{R C}$ & (Intercept) & $(1,29)$ & 65.184 & 0 & 0 & & & \\
\hline & Time & $(1,29)$ & 9.872 & 0.004 & 0.011 & Time Effect & -0.018 & $\mathrm{M} \Omega$ \\
\hline & Condition & $(1,9)$ & 3.417 & 0.098 & 0.171 & $\begin{array}{l}\text { Depolarized } \\
\text { Async Effect }\end{array}$ & -1.933 & $\mathrm{M} \Omega$ \\
\hline & Time:Condition & $(1,29)$ & 0.008 & 0.928 & 0.963 & Interaction Effect & -0.001 & $\mathrm{M} \Omega / 20$ Min. \\
\hline \multirow{2}{*}{ Vrest } & (Intercept) & $(1,39)$ & 1839.577 & 0 & 0 & & & \\
\hline & Time & $(1,39)$ & 28.236 & 0 & 0 & Time Effect & -0.085 & $\mathrm{mV}$ \\
\hline & Condition & $(1,9)$ & 0.242 & 0.634 & 0.772 & $\begin{array}{l}\text { Depolarized } \\
\text { Async Effect }\end{array}$ & 1.301 & $\mathrm{mV}$ \\
\hline & Time:Condition & $(1,39)$ & 0.011 & 0.917 & 0.963 & Interaction Effect & -0.003 & $\mathrm{mV} / 20 \mathrm{Min}$. \\
\hline
\end{tabular}




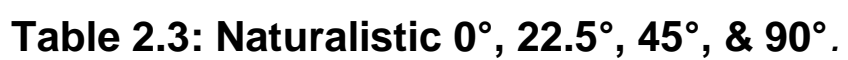

\begin{tabular}{|c|c|c|c|c|c|c|c|c|c|c|}
\hline $\begin{array}{r}\text { Dependent } \\
\text { Variable }\end{array}$ & Parameter & df & F Value & p-Value & $\begin{array}{l}\text { FDR } \\
\text { Adjusted p }\end{array}$ & Parameter & $\begin{array}{l}\text { Effect } \\
\text { Estimate }\end{array}$ & & & \\
\hline \multirow[t]{4}{*}{$\mathrm{CC}$} & (Intercept) & $(1,154)$ & 240.156 & 0 & 0 & & & & & \\
\hline & Time & $(1,154)$ & 1.329 & 0.251 & 0.37 & Time Effect & 0.001 & & & $\Delta \mathrm{mV} / \Delta \mathrm{mV}$ \\
\hline & Condition & $(3,19)$ & 0.05 & 0.985 & 0.995 & $\begin{array}{l}\text { Phase Effects }\left(0^{\circ}, 22.5^{\circ},\right. \\
\left.45^{\circ}, 90^{\circ}\right)\end{array}$ & 0.013 & -0.006 & 0.1 & $\Delta \mathrm{mV} / \Delta \mathrm{mV}$ \\
\hline & Time:Condition & $(3,154)$ & 3.246 & 0.024 & 0.053 & $\begin{array}{l}\text { Interaction Effects } \\
\text { (Time*Phase) }\end{array}$ & 0.001 & 0.001 & -0.002 & $(\Delta \mathrm{mV} / \Delta \mathrm{mV}) / 20 \mathrm{Min}$ \\
\hline \multirow[t]{4}{*}{ gj-1 } & (Intercept) & $(1,59)$ & 40.007 & 0 & 0 & & & & & \\
\hline & Time & $(1,59)$ & 0 & 0.995 & 0.995 & Time Effect & 0.006 & & & $\mathrm{M} \Omega$ \\
\hline & Condition & $(3,19)$ & 0.044 & 0.987 & 0.995 & $\begin{array}{l}\text { Phase Effects }\left(0^{\circ}, 22.5^{\circ}\right. \\
\left.45^{\circ}, 90^{\circ}\right)\end{array}$ & 0.186 & -0.001 & -0.535 & $\mathrm{M} \Omega$ \\
\hline & Time:Condition & $(3,59)$ & 1.964 & 0.129 & 0.219 & $\begin{array}{l}\text { Interaction Effects } \\
(\text { Time*Phase) }\end{array}$ & -0.014 & -0.017 & 0.007 & $\mathrm{M} \Omega / 20 \mathrm{Min}$ \\
\hline \multirow[t]{4}{*}{ R1 } & (Intercept) & $(1,78)$ & 429.125 & 0 & 0 & & & & & \\
\hline & Time & $(1,78)$ & 5.245 & 0.025 & 0.053 & Time Effect & -0.023 & & & $\mathrm{M} \Omega$ \\
\hline & Condition & $(3,19)$ & 0.177 & 0.91 & 0.995 & $\begin{array}{l}\text { Phase Effects }\left(0^{\circ}, 22.5^{\circ}\right. \\
\left.45^{\circ}, 90^{\circ}\right)\end{array}$ & 0.089 & -0.907 & -0.022 & $\mathrm{M} \Omega$ \\
\hline & Time:Condition & $(3,78)$ & 4.131 & 0.009 & 0.025 & $\begin{array}{l}\text { Interaction Effects } \\
\text { (Time*Phase) }\end{array}$ & 0.007 & 0.035 & 0.012 & $\mathrm{M} \Omega / 20 \mathrm{Min}$. \\
\hline \multirow[t]{4}{*}{ R11 } & (Intercept) & $(1,115)$ & 333.2 & 0 & 0 & & & & & \\
\hline & Time & $(1,115)$ & 1.785 & 0.184 & 0.286 & Time Effect & -0.006 & & & $\mathrm{M} \Omega$ \\
\hline & Condition & $(3,19)$ & 1.072 & 0.384 & 0.538 & $\begin{array}{l}\text { Phase Effects }\left(0^{\circ}, 22.5^{\circ},\right. \\
\left.45^{\circ}, 90^{\circ}\right)\end{array}$ & 0.456 & -0.103 & 0.316 & $\mathrm{M} \Omega$ \\
\hline & Time:Condition & $(3,115)$ & 5.067 & 0.002 & 0.008 & $\begin{array}{l}\text { Interaction Effects } \\
\text { (Time*Phase) }\end{array}$ & 0.006 & 0.013 & -0.004 & $\mathrm{M} \Omega / 20 \mathrm{Min}$ \\
\hline \multirow[t]{4}{*}{ R12 } & (Intercept) & $(1,65)$ & 92.862 & 0 & 0 & & & & & \\
\hline & Time & $(1,65)$ & 0.315 & 0.576 & 0.734 & Time Effect & -0.003 & & & $\mathrm{M} \Omega$ \\
\hline & Condition & $(3,19)$ & 0.459 & 0.714 & 0.864 & $\begin{array}{l}\text { Phase Effects }\left(0^{\circ}, 22.5^{\circ}\right. \\
\left.45^{\circ}, 90^{\circ}\right)\end{array}$ & 0.394 & -0.105 & 0.475 & $\mathrm{M} \Omega$ \\
\hline & Time:Condition & $(3,65)$ & 3.298 & 0.026 & 0.053 & $\begin{array}{l}\text { Interaction Effects } \\
(\text { Time*Phase) }\end{array}$ & 0.003 & 0.01 & -0.007 & $\mathrm{M} \Omega / 20 \mathrm{Min}$ \\
\hline \multirow[t]{2}{*}{ Rc } & (Intercept) & $(1,63)$ & 38.52 & 0 & 0 & & & & & \\
\hline & Time & $(1,63)$ & 2.671 & 0.107 & 0.2 & Time Effect & 0.009 & & & $\mathrm{M} \Omega$ \\
\hline
\end{tabular}




\begin{tabular}{|c|c|c|c|c|c|c|c|c|c|c|}
\hline & Condition & $(3,19)$ & 0.42 & 0.741 & 0.864 & $\begin{array}{l}\text { Phase Effects }\left(0^{\circ}, 22.5^{\circ} \text {, }\right. \\
\left.45^{\circ}, 90^{\circ}\right)\end{array}$ & 3.62 & 0.338 & -0.362 & $\mathrm{M} \Omega$ \\
\hline & Time:Condition & $(3,63)$ & 3.28 & 0.027 & 0.053 & $\begin{array}{l}\text { Interaction Effects } \\
\text { (Time*Phase) }\end{array}$ & -0.08 & -0.022 & 0.002 & $\mathrm{M} \Omega / 20 \mathrm{Min}$. \\
\hline \multirow[t]{4}{*}{ Vrest } & (Intercept) & $(1,75)$ & 4874.341 & 0 & 0 & & & & & \\
\hline & Time & $(1,75)$ & 10.182 & 0.002 & 0.007 & Time Effect & -0.03 & & & $\mathrm{mV}$ \\
\hline & Condition & $(3,19)$ & 0.715 & 0.555 & 0.734 & $\begin{array}{l}\text { Phase Effects }\left(0^{\circ}, 22.5^{\circ} \text {, }\right. \\
\left.45^{\circ}, 90^{\circ}\right)\end{array}$ & -2.735 & -2.222 & 0.993 & $\mathrm{mV}$ \\
\hline & Time:Condition & $(3,75)$ & 1.925 & 0.133 & 0.219 & $\begin{array}{l}\text { Interaction Effects } \\
\text { (Time*Phase) }\end{array}$ & -0.005 & -0.001 & -0.099 & $\mathrm{mV} / 20 \mathrm{Min}$. \\
\hline
\end{tabular}


Table 2.4: Individualized $0^{\circ}, 22.5^{\circ}, 45^{\circ}, 90^{\circ}, 180^{\circ}$.

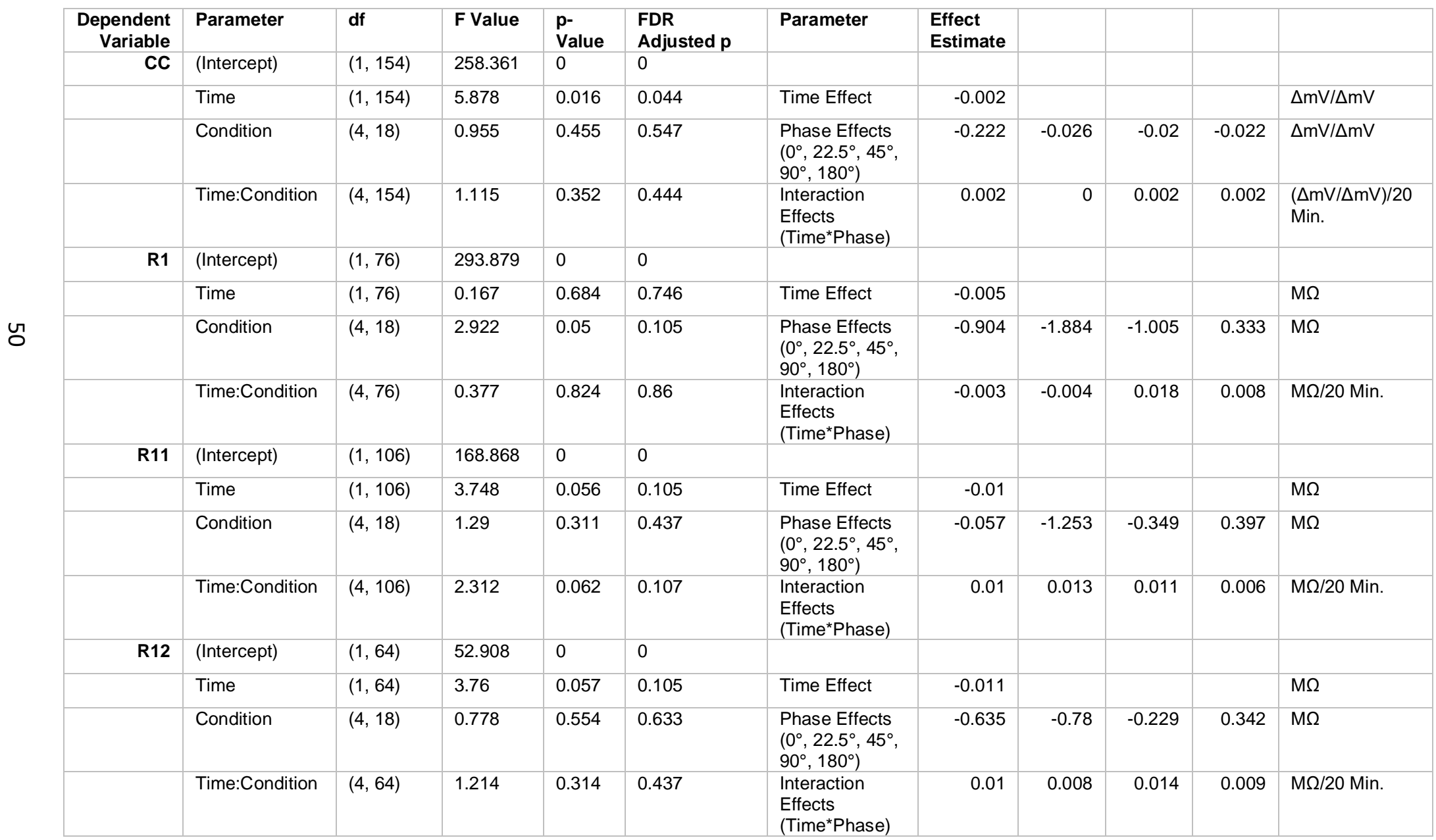




\begin{tabular}{|c|c|c|c|c|c|c|c|c|c|c|c|}
\hline Rc & (Intercept) & $(1,64)$ & 59.744 & 0 & 0 & & & & & & \\
\hline & Time & $(1,64)$ & 0.974 & 0.328 & 0.437 & Time Effect & 0.011 & & & & $\mathrm{M} \Omega$ \\
\hline & Condition & $(4,18)$ & 2.988 & 0.047 & 0.105 & $\begin{array}{l}\text { Phase Effects } \\
\left(0^{\circ}, 22.5^{\circ}, 45^{\circ} \text {, }\right. \\
\left.90^{\circ}, 180^{\circ}\right)\end{array}$ & 4.226 & -0.378 & 1.498 & 0.596 & $\mathrm{M} \Omega$ \\
\hline & Time:Condition & $(4,64)$ & 1.942 & 0.114 & 0.183 & $\begin{array}{l}\text { Interaction } \\
\text { Effects } \\
\text { (Time*Phase) }\end{array}$ & -0.017 & -0.007 & -0.017 & -0.012 & $\mathrm{M} \Omega / 20 \mathrm{Min}$. \\
\hline \multirow[t]{4}{*}{ Vrest } & (Intercept) & $(1,72)$ & 4304.873 & 0 & 0 & & & & & & \\
\hline & Time & $(1,72)$ & 108.367 & 0 & 0 & Time Effect & -0.116 & & & & $\mathrm{mV}$ \\
\hline & Condition & $(4,18)$ & 5.176 & 0.006 & 0.018 & $\begin{array}{l}\text { Phase Effects } \\
\left(0^{\circ}, 22.5^{\circ}, 45^{\circ},\right. \\
\left.90^{\circ}, 180^{\circ}\right)\end{array}$ & -8.821 & 0.971 & 3.187 & -4.328 & $\mathrm{mV}$ \\
\hline & Time:Condition & $(4,72)$ & 0.291 & 0.883 & 0.883 & $\begin{array}{l}\text { Interaction } \\
\text { Effects } \\
\text { (Time*Phase) }\end{array}$ & 0.006 & -0.019 & -0.01 & 0.017 & $\mathrm{mV} / 20 \mathrm{Min}$. \\
\hline
\end{tabular}




\title{
Chapter 3
}

\section{Effects of blockade of potassium currents on membrane conductance and channel expression at 1 hour and 24 hours in motor neurons of the cardiac ganglion}

\begin{abstract}
Crustacean cardiac ganglion motor neurons are robust to disrupted activity, displaying rapid compensatory changes following hyperexcitability. Stabilization of output following pharmacological reduction of high-threshold potassium conductance $\left(\mathrm{I}_{\mathrm{HTK}}\right)$ occurs through increase in non-blocked potassium conductances after approximately 1 hour. Whether these changes persist or are altered at longer time scales is unknown as is whether transcriptional changes occur alongside the post translational changes documented. We induce a reduction of high-threshold potassium currents for 1 or 24 hours and measure mRNA abundance of ion channel and innexin transcripts, membrane properties and currents, and cell output with a standardized fictive bursting protocol. We find evidence of changing mRNA relationships with fast $(<=1$ hour $)$ and slower $(<=24 \mathrm{~h})$ onset. Rapid changes in mRNA abundance are seen for the shal transcript, which encodes an A-type potassium channel, and inx2, which encodes an innexin. We find changes in membrane properties at 24 hours including an increase in membrane resistance, and an increase in the A-type potassium current at $0 \mathrm{mV}$ and slope of activation (nA/mV). At 24 hours motor neurons exhibit hyperexcitability, depolarizing to higher amplitudes and exhibiting a greater area under the curve of the depolarization.
\end{abstract}


These results suggest hyperexcitability induces rapid expression changes which do not attain steady state transcriptional relationships with the same speed. Physiological changes continue even following apparent output compensation. Absent changes in synaptic conductance or other properties motor neurons fail to maintain output excitability 24 hours after the initial blockade. 


\section{Significance Statement}

To produce motor patterns necessary for survival (e.g., breathing, chewing, locomotion) neural networks generate and maintain output despite changing physiological and environmental factors. Understanding how these systems achieve robustness requires understanding how the constituent neurons respond to deviations from normal activity - with either in compensatory or pathological effects. In this system, pharmacologically induced hyperexcitability through reduction of a potassium current has been shown to result in increased conductance of a non-blocked potassium current and reduction of hyperexcitability. Whether hyperexcitability induces solely posttranslational changes or whether transcriptional changes occur, co-incident or on a longer time scale, has not been explored. We show evidence indicating altered correlations following perturbation and report measures of excitability, membrane properties, and transcript abundances. These data suggest hyperexcitability induces rapid transcriptional changes in abundance and correlations. Furthermore, we find an apparent inability to maintain excitability through cell specific changes after 24 hours of perturbation, suggesting a change in network properties may be required.

\section{Introduction}

The activity of a neuron is dictated by the relative balance of ionic conductances and the input which it receives through neuromodulators and chemical or electrical synapses. Disruption of this balance through modulation (Lane et al., 2018), blockade (Ransdell et al., 2012b), or change in reversal potential (He et al., 2020) can lead to hyperexcitability and disrupted output. While some conductance relationships have been shown to be activity independent (MacLean et al., 2003, 2005), robust activity dependent changes in cell properties have been observed following increased activity (Ransdell et al., 2012b; He et al., 2020) or removal of activity (Turrigiano and Marder, 1993; 
Turrigiano et al., 1995; Thoby-Brisson and Simmers, 1998). Modeling suggests that cell activity, sensed by intracellular calcium, could result in homeostatic compensation and (O'Leary et al., 2013a) through transcriptional changes leading to correlations reminiscent to those seen in nature between transcript abundances (Schulz et al., 2007b), and ionic conductances (Khorkova and Golowasch, 2007b). This is supported by a recent study (Santin and Schulz, 2019a) demonstrating that membrane voltage is sufficient to maintain the majority of correlations between channel mRNAs following removal of excitatory input. Little is known about the effect of increased activity on the maintenance or modification of transcript relationships, the time course thereof, and the persistence or impermanence of membrane changes with a prolonged excitatory perturbation.

In the Cancer borealis cardiac ganglion, bath application of tetraethylammonium (TEA) to the motor neurons (Large Cells, LCs) blocks the calcium activated potassium current, $I_{\mathrm{KCa}}$, the primary component of the high threshold potassium current $\mathrm{I}_{\mathrm{HTK}}$. Blockade results in hyperexcitability, increased burst duration (Ransdell et al., 2012b), total depolarization, burst amplitude, spikes per burst (Lane et al., 2016) and a loss of synchrony between motor neurons (Ransdell, 2013a). These effects are ameliorated through an increase in the A-type potassium current, $\mathrm{I}_{\mathrm{A}}$,(Ransdell et al., 2012b) and increased coupling between motor neurons (Lane et al., 2016). Whether homeostatic tuning of transcript relationships or abundances occurs following hyperexcitability or if post-translational changes are sufficient to mitigate hyperexcitability is not known.

In this present study we explore the transcriptional and post-transcriptional changes associated with hyperexcitability and the time course of these changes. We aim to determine first if there are transcriptional changes occurring along with the previously documented post-translational changes and second whether compensatory changes are 
stable and effective on the scale of hours (i.e. 24 hours later). Initially, we establish evidence for a change in the relationships mRNAs encoding ion channels and innexins following exposure to TEA. We sample cells never exposed to TEA (0 hours TEA), exposed to 1 hour of TEA, or following 24 hours of exposure and examined the correlations in expression at each time point. We repeated this experiment collecting a second set of mRNA abundance measurements and physiological measurements from the same cells. This enables us to establish whether rapid physiological changes are maintained at 24 hours or if other compensatory changes occur on this longer timescale. Using a standardized excitatory post-synaptic potential (EPSP) mimicking current injection we assess cellular excitability at each time point to determine whether excitability is increased relative to control or maintained, providing evidence of effective compensation.

\section{Materials and Methods}

\section{Animals}

Adult male Jonah crabs (Cancer borealis) were purchased from The Fresh Lobster Company (Gloucester, MA) and housed in tanks of $12^{\circ} \mathrm{C}$ artificial seawater. Prior to use the crabs were anesthetized by thirty minutes of cold exposure. The heart was then removed, and the ganglion isolated through microdissection and pinned in a Sylgard lined petri dish. The connective sheath around the motor neurons was removed with a fine pin to allow for intracellular recordings and ultimately removal of the neuron. During and following fine dissection, the preparation was bathed in chilled physiological saline $(440 \mathrm{mM} \mathrm{NaCl}, 26 \mathrm{mM} \mathrm{MgCl} 2,13 \mathrm{mM} \mathrm{CaCl} 2,11 \mathrm{mM} \mathrm{KCl}$, and $10 \mathrm{mM}$ HEPES at a pH of 7.4-7.5). Unless otherwise stated, all chemicals used were purchased from Fisher Scientific. 
The $C$. borealis cardiac ganglion consists of a pacemaking kernel of four interneurons referred to as "small cells" (SCs) which excite five motor neurons termed "large cells" (LCs). Two LCs (LCs 1 and 2) reside in the posterior of the network near the small cells. This region was surrounded by a petroleum jelly well thereby isolating a the SCs and a section of trunk containing projections to and from the anterior large cells (LCs 3, 4, 5). We recorded extracellular activity from this location using a differential AC amplifier (A-M Systems model 1700) and intracellular recordings from the anterior large cells (LC3, 4, and 5) with sharp electrodes filled with $3 \mathrm{M} \mathrm{KCl} \mathrm{(6-15} \mathrm{M \Omega ).} \mathrm{All} \mathrm{protocols}$ were run, and data collected with Clampex 10.7 (Molecular Devices, San Jose CA) using Axoclamp-2A intracellular amplifiers (Axon Instruments), a Brownlee precision amplifier (Model 410) (which preamplified the current injecting channels) and a Digidata 1440A digitizer (Molecular Devices).

In the experimental groups we exposed LCs to tetraethylammonium (TEA) at a concentration of $25 \mathrm{mM}$ for 1 or 24 hours, reducing the transient portion of the high threshold potassium current ( $\mathrm{I}_{\mathrm{HTK}}$ ) by $~ 92 \%$ (Ransdell et al., 2013a). The petroleum jelly well surrounding the posterior of the network served to prevent exposure of the SCs to TEA. To the well for leaks and to hedge against slow leaks several drops of control saline were added to the well. In the 1 -hour condition we kept the preparation $\sim 12^{\circ} \mathrm{C}$ on the electrophysiology rig. For the 24 -hour condition the preparation dish was covered with a petri dish lid or film to prevent evaporation and placed in an incubator set to $12^{\circ} \mathrm{C}$.

\section{Electrophysiology}

\section{Membrane Resistance and lonic Conductances}

We suspended network activity by displacing the physiological saline in the interneuron containing well with isotonic sucrose $(750 \mathrm{mM})$ as described previously 
(Lane et al., 2016). In the silenced network we measured input resistance $\left(R_{\text {in }}=\frac{\Delta V_{1}}{I_{1}}\right)$ using negative current pulses. When possible, we recorded from strongly electrically coupled LCs simultaneously so that we would be able to calculate transfer resistance $\left(\mathrm{R}_{\text {transfer }}=\frac{\Delta \mathrm{V}_{2}}{\mathrm{I}_{1}}\right)$ which enables us to calculate membrane resistance $\left(\mathrm{R}_{\text {mem }}=\right.$ $\left.\frac{R_{\text {in } 1} \cdot R_{i n 2}-R_{12}^{2}}{R_{\text {in } 2}-R_{12}}\right)$ (Bennett, 1966b). Here and subscript 1 and 2 refer to the pre- and post synaptic LCs with respect to the electrical synapse. We injected $0 \mathrm{nA}(250 \mathrm{~ms}),-6 \mathrm{nA}$ (1500 ms), and $0 \mathrm{nA}$ (2999 ms) into one cell and $0 \mathrm{nA}$ (2999 ms), -6 nA (1500 ms), and $0 \mathrm{nA}(250 \mathrm{~ms})$ into the other five times. Next, we voltage clamped high threshold and Atype potassium currents. High threshold potassium ( $\left.\mathrm{I}_{\mathrm{HTK}}\right)$ we measure with $5 \mathrm{mV}$ steps from $-55 m V$ to $+20 m V$ from a holding potential of $-40 m V$. A-type potassium we measure by using an identical protocol, save the holding potential is $-80 \mathrm{mV}$, and subtracting $\mathrm{I}_{\mathrm{HTK}}$ from the resultant trace. These protocols are based on previously published methods (Ransdell et al., 2012b). We leak subtracted $\mathrm{I}_{\text {HTK }}$ based on a linear fit of the voltage steps between $-39 \mathrm{mV}$ and $-81 \mathrm{mV}$. We obtained linear fits for the approximately linear region of the activations of $\mathrm{I}_{\mathrm{HTK}}$ and $\mathrm{I}_{\mathrm{A}}$ between $-35 \mathrm{mV}$ and $+5 \mathrm{mV}$ for both the inactivating transient peak and non-inactivating current. We consider both resultant slope and intercept in our analysis.

\section{Current clamp stimulus protocol}

When exposed to TEA, current injections can produce plateau like depolarizations (Ransdell et al., 2012b). When excitatory post-synaptic potential (EPSP) mimicking protocol was used responses similar to those within an active TEA exposed network were observed (Ransdell et al., 2013a). We apply the same EPSP mimicking protocol at each cell's resting membrane potential. This enables systematic comparison 
of the excitability of the cell. The protocol contains four sweeps of four fictive bursts over 19.687 seconds (78.748 seconds total per file).

From these recordings extract measures previously used to quantify LC excitability: the maximum amplitude, total depolarization (i.e. the area under the curve (AUC) relative to resting membrane potential) (Lane et al., 2016), and the correlation coefficient (Ransdell et al., 2013a). In previous studies correlation coefficient has been calculated between LC pairs (Ransdell et al., 2013a; Lane et al., 2016). This captures hyperexcitability only in so far as TEA induced hyperexcitability leads to desynchronization through unmasked current variability (Ransdell et al., 2013a). For the present study we calculate correlation coefficient not relative to another LC's voltage but to a predicted voltage response. We predicted each cell's voltage response based on its membrane resistance and a tau of $25 \mathrm{~ms}$ with a simple model written for Brian 2 (Stimberg et al., 2019). This enables subtraction of the approximate effect of membrane resistance although it does not allow us to account for differential activation of currents as a result of an elevated voltage response. We consider correlation between the observed response and predicted response, maximum amplitude, AUC maximum amplitude less the predicted amplitude, and the AUC less the predicted AUC. For the purposes of calculating AUC, resting voltage is defined as the average of the lowest $20 \%$ of voltage samples. To account for the variability across sweeps, for each measure we calculate the inner quartile range and the median for each cell, running statistics on both.

\section{Molecular Biology}

Collection of Large Cells 
Following collection of physiological data, the LCs were enclosed in a petroleum jelly well filled with $\sim 2.5 \mathrm{mg} / \mathrm{ml}$ protease (Sigma - P6911, St. Louis, MO, USA) in saline. After digestion of the connective tissue the protease was washed out with copious chilled saline. Next, over the course of $\sim 15$ minutes $70 \%$ ethylene glycol in crab saline was added dropwise until the saline was fully replaced. If needed, the preparation was placed in a freezer set to $-20^{\circ} \mathrm{C}$ until cold. LCs were then hand dissected using fine forceps, which were cleaned prior to each cell's collection. Each LC was placed in a collection tube containing $400 \mu \mathrm{l}$ of lysis buffer (Zymo Research, Irvine, CA, USA) and stored at $-80^{\circ} \mathrm{C}$.

\section{Pre-amplification}

In the replication, but not the pilot experiment, we used the following process to pre-amplify our cDNA targets. Using the Quick-RNA MicroPrep kit (Zymo Research, Irvine, CA, USA) we isolated each cell's total RNA and reverse transcribed the total RNA using oligo-dT and random hexamer primers (qScript cDNA Supermix; QuantaBio, Beverly, MA, USA). Half of the resultant cDNA was pre-amplified with PerfeCTa PreAmp Supermix (QuantaBio, Beverly, MA, USA) by following the manufacture's protocol for a $20 \mu \mathrm{L}$ reaction volume. We used a 14 -cycle PCR reaction with target-specific primers. Following pre-amplification, we diluted the cDNA samples $7.5 \mathrm{x}$ with nuclease-free water arriving at a final volume of $150 \mu \mathrm{L}$.

\section{Multiplex Primers}

In this study we consider the abundance of ion channel and innexin mRNAs.

Specifically, we measure voltage gated $\mathrm{Na}+$ channel (nav), L-type Ca2+ channel (cav1), P/Q-type Ca2+ channel (cav2), large conductance Ca2+ activated K+ channel (bkkca), 
A-type K+ channel (shaker, shal), delayed-rectifying K+ channel (shab), delayedrectifying $\mathrm{K}+$ channel (shaw1, shaw2), and robustly expressed innexins (inx1, inx2, inx3).

We used LGC Biosearch Technologies (Teddington, UK) qPCR Assay Design Software, RealTimeDesign ${ }^{\mathrm{TM}}$, to design our assays based on previously determined open reading frames (Schulz et al., 2007b) or the $C$. borealis nervous system transcriptome (Northcutt et al., 2016a). Table 3.S1 records the specific primers, probe sequences, and fluorophores used.

\section{Quantitative Polymerase Chain Reaction}

$2.5 \mu \mathrm{L}$ of pre-amplified cDNA was used for each $10 \mu \mathrm{L}$ qPCR reactions using PerfeCTa Multiplex qPCR ToughMix as indicated by the manufacturer's instructions (5X, QuantaBio, Beverly, MA, USA). Multiplex reactions (Table 3.S1) were run on 96-well plates in triplicate using a CFX96 Touch ${ }^{\mathrm{TM}}$ Real-Time PCR Detection System from BioRad (Hercules, CA, USA). qPCR protocol was as follows: 3 minutes at $95^{\circ} \mathrm{C}$ followed by 40 cycles 15 seconds at $95^{\circ} \mathrm{C}$ followed by 1 minute at $58^{\circ} \mathrm{C}$, fluorescence being measured at the end of each cycle. Quantitation cycle $(\mathrm{Cq})$ was converted to an absolute copy number for each gene by interpolating based on a standard curve generated from known copy numbers (106-101 copies). We corrected for the sample quantity and pre-amplification as well.

\section{Experimental Design and Statistical Analysis}

\section{Experimental Design}

We designed a pilot study to assess whether TEA induced plasticity occurs solely post transcriptionally or if mRNA expression is changed as well. We collected LCs without exposure to TEA $(n=15)$, after 1 hour exposure $(n=15)$, and after 24 hours of exposure $(n=13)$. Due to concern of batch effects, we do not examine the mean 
differences between groups and have examined only the correlations between 11 transcripts of interest and find support for a strengthening of correlations at 24 hours.

We sought to build off this pilot by measuring membrane currents and cell excitability prior to cell collection. We used the same time points ( 0 hours $n=21,1$ hour $\mathrm{n}=17,24$ hours $\mathrm{n}=22$ ) allowing for comparison of the correlations across these replicates. We processed these data minimizing the potential for batch effects and allow for comparison of mean changes across these groups. With the physiological collected from each cell we are able to examine co-incident changes with incubation in TEA. None of these experiments were preregistered. Sample size was based on sample size used in similar studies rather than a power calculation.

\section{Data preprocessing}

Physiological measurements (excluding those for excitability) were obtained using Clampfit 10.7 and aggregated using Excel before processing in $\mathrm{R}$ (Wickham and Bryan, 2019). Metrics for excitability were calculated programmatically with custom written functions in $\mathrm{R}$. This process was aided immensely by the readABF library (Syekirin, 2020). Before statistical modeling outliers were removed. Observations were classified as outliers if they fell outside 1.5 times the inner quartile range above or below the median and were systematically excluded.

\section{Statistical Analysis}

All statistics were run using R. Correlation coefficients shown are Pearson's correlation coefficients $(\mathrm{R})$. When visualized as a correlogram the diameter of the point representing each correlation is equal to the absolute value of the correlation. The set of pairwise correlations for each time point were compared as cumulative probability distributions using the Kolmogorov-Smirnov test. In evaluating the consistency of 
correlations between trials we used a fixed cutoff of $R>=0.7$ to define a "strong" positive correlation. A tally of the number of strong correlations seen at a given time point in both, one, or neither trial is shown in Table 3.1. The $\mathrm{R}$ values themselves are reported in Table 3.2, with those above 0.7 highlighted. Within the replicate trial, we tested for a change in the slope of the linear relationship between conditions using an ANCOVA. The adjusted $p$ values, corrected using a false discovery rate, for the interaction term are reported in Table 3.3. We also report the correlation coefficients and adjusted $p$ values for the same in Table 3.3, with those conditions and time points with a significant correlation highlighted. We tested for univariate effects using a one-way ANOVA. We calculated both asymptotic p-values and empirical p-values resampling 1000 times. The resultant $p$ values are often very similar but the latter method is more robust to deviations from the assumed distribution. To limit the family wise error rate, we corrected the empirical $p$ values using a false discovery rate. The corrected values are denoted as " $p_{a d j}$ ". We consider an effect as "significant" if $p_{a d j}$ is less than 0.05 . We use Tukey's Honest Significant Difference to compare groups where there was a significant main effect. The code written for these analyses and the data used are available upon request. Please write to the corresponding author.

\section{Results}

TEA induced hyperexcitability results in changes to transcriptional relationships

Tetraethylammonium (TEA) has been shown to induce hyperexcitability in LCs (Ransdell et al., 2012b) and decrease both waveform similarity (Ransdell et al., 2013a) and synchrony (Lane et al., 2016) between cells. Following approximately 60 minutes of exposure to TEA, LCs have been shown to exhibit homeostatic plasticity; reducing hyperexcitability coincident with an increase in $I_{A}$ (Ransdell et al., 2012b) and coupling 
conductance which reduces the loss of synchrony (Lane et al., 2016). The compensatory changes explored to date have focused solely on post-transcriptional changes. Here, we examine a longer time point and measure the abundance of ion channel and innexin mRNAs to establish whether additional compensatory changes occur, whether compensation is stable after 24 hours, and if the abundances or relationships of transcripts are altered as well.

We exposed anterior LCs to TEA for 0 (i.e., unexposed), 1, or 24 hours and measured transcripts all of which encode ion channels or gap junction proteins. Due to evidence of batch effects, we restrict our analysis to correlations between transcripts rather than mean expression changes for these data (denoted as "Pilot"). We find an increase in the number of strong positive correlations at 1 hour relative to 0 hours and considerably more at 24 hours relative to 0 hours (Figure $3.1 \mathrm{~A}$, top row). The three innexin transcripts (inx1, inx2, inx3) exhibit strong positive correlations at all time points. Increased positive correlations appear to not be constrained to transcripts of channels selective to a given ion species, or those associated with hyperpolarization or depolarization. While we do observe more positive correlations between some directionally aligned currents (see nav vs cav2, cav1 vs cav2, and shal vs bkkca), transcripts of antagonistic currents increase as well (see shal vs cav2). This is inconsistent with our initial expectations - that transcripts of hyperpolarizing currents would become more positively correlated with each other and more weakly correlated with transcripts of depolarizing currents.

With clear evidence of change over time but inability to establish changes in transcript abundance we repeated this experiment (denoted as "Replicate"), also collecting excitability measurements, active properties, and passive properties from each cell. Unlike in the pilot data, we find few negative correlations and no strong negative 
correlations at any of the time points (Figure 3.1 A, bottom row). While there still appear to be more strong positive correlations at 1 and 24 hours than at 0 hours, changes appear do not fully agree pilot data.

Examining the empirical cumulative distribution functions (ECDFs) for the sets of correlations (Figure 3.1 B) we find no significant difference in either trial between the distributions of correlations at 0 and 1 hour (Pilot Oh vs 1 h KS test $p=0.438$, Replicate Oh vs $1 \mathrm{~h}$ KS test $p=0.438$ ). Between 0 hours vs 24 hours there is a significant right shift relative to 0 (Pilot $0 \mathrm{~h}$ vs $24 \mathrm{~h}$ KS test $p<0.001$ ) that is not seen in the replicate (Replicate Oh vs $24 \mathrm{~h} \mathrm{KS}$ test $p=0.226$ ). Despite this the distribution of correlations above approximately 0.5 shows little divergence in either trial. Contrasting 1 and 24 hours, both datasets suggest these distributions are inequivalent (Pilot $1 \mathrm{~h}$ vs $24 \mathrm{~h}$ KS test $p=0.001$, Replicate $1 \mathrm{~h}$ vs $24 \mathrm{~h} \mathrm{KS}$ test $\mathrm{p}=0.025$ ) but in replicate 1 the 24 -hour group is more positive whereas the 1 -hour group is more positive in replicate 2 . In both replicates the differences between ECDFs are minor for strong positive correlations $(R>=0.7)$.

To examine the change of individual correlations rather than the distribution of correlations we calculated Pearson's correlation coefficient and ordinary least squares regression on the set of correlations over each pair of time points (Figure 3.1 C). We find that the correlation of correlations is higher and the slope of the line of best fit is closer to one in the replicate than in the pilot. Thus, correlations in the replicate are more consistent from one time point to another than in the pilot.

The pilot data shows far greater dispersion than the replicate. However, there appear to be clusters of strong positively correlated transcripts which appear to be stable across time in both the pilot and replicate. We compared the correlations at each time point to determine how many were "strong" positive $(R>=0.7)$ correlations in both groups. 
Of the 66 correlations considered, at 0 hours 6 were strongly positive in both trials, 13 in only one of the two. At 1 hour 5 strongly positive correlations were found in both, 16 in only one. At 24 hours these increased to 8 in both, 21 in only one (Table 3.1). Examining the specific identities of these correlation (Table 3.2) we find many correlations with general agreement across trials (e.g., cav1 vs shab, cav1 vs shaker, shab vs shaw1, inx1 vs inx2, inx1 vs inx3, inx2 vs inx3, shaker vs shab), some elevated predominantly in one trial (e.g., cav1 vs shaw, cav2 vs shab, shaker vs shaw1), and several over thresholds at a single time point and trial (e.g., nav vs cav1, nav vs shab, shaker vs shal).

In the replicate dataset we examined the slopes of relationships between transcript abundances and correlations across time points. We used an ANCOVA to examine the former, Pearson's R for the latter. Due to the non-overlap in some correlations between the pilot and replicate we adjust $p$ values for the ANCOVA interaction term and correlations as described in the methods. After correction we find no significant change in slopes for any of the pairs tested. We find 14 correlations significant for each time point sampled (nav vs bkkca, nav vs shal, cav1 vs cav2, cav1 vs shaker, cav1 vs shab, cav1 vs shaw1, cav1 vs shaw2, cav2 vs shaker, cav2 vs shab, shaker vs shab, shaker vs shaw1, inx1 vs inx2, inx1 vs inx3, inx2 vs inx3), 5 correlations significant after one hour (cav2 vs shaw1, shaker vs shaw2, shab vs shaw1, shab vs shaw2, shaw1 vs shaw2), 4 correlations significant only in control (bkkca vs shal, bkkca vs inx1, bkkca vs inx2, bkkca vs inx3), 6 correlations significant only at one hour (cav1 vs inx2, cav2 vs bkkca, cav2 vs inx2, shakervs inx2, shal vs shaw1, shaw1 vs inx2) and 2 correlations significant only at 24 hours (cav1 vs inx3, cav2 vs shaw2). 35 correlations were not significant at any time point.

Transcript abundances reveal limited changes following TEA exposure. 
To examine whether transcript abundance was altered, we performed an ANOVA for each transcript both as an asymptotic test and though resampling with 1000 rounds of shuffling to produce and empirical p-value. The latter approach is more robust but often produces a p value effectively the same as that of the asymptotic test. We then applied Tukey's HSD as a post hoc and correct for multiple comparisons using a false discovery rate. We find no significant effect for voltage activated sodium (Figure 3.2 A) or calcium (Figure 3.2 B \& C) channel transcripts. Likewise, we find no significant change in bkkca (Figure 3.2 D) which encodes a calcium activated potassium channel. Within $\mathrm{I}_{\mathrm{A}}$ encoding shaker and shal we find no significant change in shaker expression (Figure 3.2 E) but do find a significant main effect for shal (ep adjusted $=0.019$ ), with 1 hour being significantly elevated relative to 0 and 24 hours (Figure $3.2 \mathrm{~F}$ ). For the transcripts we measured associated with $I_{k d}$ we do find a significant main effect for shab (ep adjusted $=0.046$ ) (Figure $3.2 \mathrm{G}$ ), but none in the HSD, and no significant main effects for shaw1 or shaw2 (Figure $3.2 \mathrm{H} \& \mathrm{I}$ ). Within the innexins we find a significant effect on inx2 (ep adjusted $=0.007$ ), being significantly increased at 1 and 24 hours relative to 0 hours (Figure $3.2 \mathrm{~K}$ ) and no significant effects for inx1 or inx3 (Figure $3.2 \mathrm{~J}$ \& L). The statistics presented here are summarized in Table 3.4.

Large cells do not maintain constant membrane properties over 24-hours of exposure.

Given that ionic conductance can be modulated by channel insertion or removal from the membrane or by phosphorylation of channels within the membrane, we sought to establish whether changes in post-translation changes were occurring at 24 hours relative to the previous time points. Given the few mean expression changes seen in our data we considered the possibility that changes at 1 hour might sufficiently attenuate the perturbed activity. Based on this, we predicted that membrane resistance $\left(R_{m e m}\right)$, the 
parameters describing $I_{A}$, and the metrics for excitability detailed above would form two post hoc groups separating control from 1 and 24 hours. The parameters describing $I_{\text {HTK }}$ would be expected to show the reverse - It would decrease following TEA application and remain depressed for the duration of the experiment.

We report a change in the input resistance (ep adjusted <0.001) significantly increasing at 24 hours relative to 0 (Figure 3.3 A). Membrane resistance (ep adjusted $=$ 0.037 ) increases as well, with 24 hours being significantly higher than 0 or 1 hour (Figure 3.3 B). The slope and intercept of the approximately linear region of the $I_{H T K}$ decreases and remains so for the transient (ep adjusted < 0.001) (Figure 3.3 C \& D) and sustained portion of the current (ep adjusted $<0.001$ ), consistent with effective blockade thought TEA. In measures of $I_{A}$, we find a significant increase in the intercept (ep adjusted = 0.003 ) and slope (ep adjusted $=0.003$ ) of the transient region (Figure $3.3 E \& F$ ) of $I_{A}$ but this increase occurs by 24 hours instead of by 1 hour. The sustained region of $I_{A}$ where the intercept decreases by 1 hour and recovers by 24 hours (ep adjusted $<0.001$ ). The slope of activation of the sustained portion changes significantly as well (ep adjusted $<$ $0.001)$. It decreases at 1 hour and regresses at 24 but remains statistically distinguishable from 0 and 1 hour at the terminal time point. The last membrane property we examined was resting potential. We find a significant effect of the treatment on $\mathrm{V}_{\text {rest }}$ (ep adjusted $=0.011$ ) however neither 1 hour nor 24 hours are statistically distinguishable from control (Figure 3.3 G). In summary, we find a significant increase in the intercept and slope of $I_{A}$ but later than would be expected base on previous work. We also report an increase in membrane resistance, likewise, manifesting at 24 hours. The statistics presented here are summarized in Table 3.4.

Large cells fail to maintain constant excitability across 24 hours. 
Given the increase in $\mathrm{I}_{\mathrm{A}}$ by 24 hours but not 1 hour, and the upregulation of inx2 we predicted the excitability measures would indicate a failure to maintain control excitability at one hour and a regression to control excitability at 24 hours. As the membrane resistance increased by 24 hours, and the EPSP mimetic protocol's current is not scaled relative to membrane resistance we hypothesized these differences would increase, or only be detected, following simulation-based correction for membrane resistance.

This does not appear to be the case. We find that he correlation of the voltage response and predicted (i.e. simulated) response is significantly altered (ep $=0.027$ ) being significantly reduced at 1 hour relative to control (Figure 3.4 A). The variability in correlation for a given cell increases and is significantly different at the same time point (Figure 3.4 B). The baseline voltage during these protocols is not significantly changed over time (Figure $3.4 \mathrm{C}$ ). The variability of a given cell's baseline decreases significantly at 1 and 24 hours relative to control (Figure 3.4 D). We find that the peak amplitude reached by a cell is significantly altered (ep adjusted $<0.001$ ) being higher following 24 hours than at control or 1 hour (Figure 3.4 E). The variability in amplitude decreases with the same time course (Figure 3.4 F). In addition to reaching a significantly higher amplitude, we find the area under the curve (AUC) is significantly (ep adjusted $<0.001$ ) elevated at 24 hours relative to control and 1 hour (Figure $3.4 \mathrm{G}$ ). The variability of a cell's AUC increases as well with 24 hours being significantly different from control (Figure $3.4 \mathrm{H}$ ).

The differential amplitude (i.e. following subtraction of the predicted response) follows the same pattern as the measured amplitude being significantly different (ep adjusted $=0.005$ ) across conditions with the 24 hour group being elevated relative to the rest (Figure 3.4 I). The variability of the differential amplitude is not significantly different 
across conditions (Figure $3.4 \mathrm{~J}$ ). We find no significant differences in differential AUC (Figure $3.4 \mathrm{~K}$ ) nor in the variability thereof (Figure 3.4 L). Simulated amplitude (Figure 3.4 M) and simulated AUC (Figure $3.4 \mathrm{~N}$ ) were not significantly different across conditions. The statistics presented here are summarized in Table 3.4.

\section{Discussion}

This study aims to establish whether transcriptional changes occur in response to induced hyperexcitability or whether the homeostatic response is constrained to previously identified post-translational changes (Ransdell et al., 2012b; Lane et al., 2016). The second goal of this study was to examine the time scale of LCs' plasticity determining both if cells successfully maintained excitability and whether rapid ( $\sim 1$ hour) changes persist or are altered by changes operating on a longer time scale.

\section{Activity Induced Rapid Transcriptional Plasticity}

In both the pilot and replication, we find evidence of changing correlation relationships after 1 hour and 24 hours of exposure to TEA (Figure $3.1 \mathrm{~A}$ ). This is accompanied by a right shift in the cumulative distribution of the correlations at 1 hour relative to control. We do not observe the same shifts in distribution across experiments for Control relative to 24 hours or 1 hour relative to 24 and among the stronger positive correlations there is less distance between the two distributions (Figure 3.1 B). Furthermore, we find that the correlations in the replication are more consistent across time points -- the correlation of correlations is higher and the regression slope closer to 1 (Figure $3.1 \mathrm{C})$. We find many correlations are high $(\mathrm{R}>0.7)$ in both groups for a given time point (Table 3.1). Examining individual correlations, we find many that are high at specific time points in both trials (e.g. shaker vs shab, inx1 vs inx2) suggesting some repeatability in these relationships but also find strong correlations only at select time 
points in the pilot (e.g. shal vs inx1) or replicate (shaker vs shaw1) (Table 3.2). As the aim of the replicate data set was to avoid the confounding effects in the pilot, we focus our analysis on the changes within the correlations of the replicate, using an adjusted $p$ value rather than a correlation cut off to establish relevance.

Of the correlations between channel transcripts only bkkca vs shal is present in control and then lost subsequently. Correlations between transcripts associated with $I_{K d}$ (shab, shaw1, shaw2) strengthen and become significant after 1 hour. This also occurs for one of the calcium channels measured, cav2 and shaw1. In addition to persisting changes, we observe transient correlations significant at only 1 hour between a shal and shaw1 and cav2 and bkkca. These changes are suggestive of short and long term compensatory changes in transcriptional relationships.

We documented several significant correlations between innexin and channel transcripts. Little is known about the potential coregulation of these features. Perhaps the most tantalizing interaction suggested is between $\mathrm{I}_{\mathrm{Ca}}, \mathrm{I}_{\mathrm{HTK}}$, and coupling conductance. Intracellular calcium has been shown to be necessary for the homeostatic increase of $I_{\text {HTK }}$ (Ransdell et al., 2012b) and has also been implicated in regulation of coupling strength (Kick and Schulz 2021). Support for coregulation of $I_{H T K}$ and coupling has been shown (Ransdell, 2013a), but this has not been confirmed. We find a significant positive correlation between cav2 vs inx2 and cav2 vs bkkca at 1 hour incubation. This finding makes the cardiac ganglion a promising system to substantiate or disconfirm coregulation of coupling and ionic conductances either through transcriptional or post-translational effects.

Beyond bivariate relationship change we found that two genes (shal, inx2) which exhibited significant differences in abundance between control and 1 hour. At 24 hours, shal expression regresses to be indistinguishable from control whereas inx 2 remains 
elevated for the duration of the experiment. We note that shal encodes an A-type potassium channel and measured I $I_{A}$ 's intercept and slope to increase at 24 hours of exposure. Additional study will be required to determine whether the decrease in abundance at 24 hours is due to negative feedback from increased protein abundance, a change in activity, or some other cause.

\section{Differential Excitability and Membrane Properties After 24 Hours}

Beyond changes in ion channel transcript abundances and abundance relationships we find changes in the membrane properties and excitability of the cells at 1 and 24 hours. Unlike in previous studies which have reported data from repeated measurements between 0 and 1 hour (Ransdell et al., 2012b; Lane et al., 2016) we find no significant increase in the $\mathrm{I}_{\mathrm{A}}$ transient at 1 hour. At 24 hours we observe an increase in the slope and intercept of the transient (Figure 3.3 E \& F). We also observed an increase in the intercept of the sustained portion of $I_{A}$ at 24 hours, but a reduction at 1 hour. We find a significant increase in membrane and input resistance between control and 24 hours, being indistinguishable from control $\left(R_{\text {in }}\right)$ or indistinguishable from either group $\left(R_{\text {mem }}\right)$ (Figure 3.3 A \& B). These findings are suggestive of activity induced plasticity in response to hyperexcitability which requires longer timescales to observe than were used in previous studies.

To systematically assay the excitability of LCs we stimulated each cell with an EPSP mimicking protocol, simulated the expected response for a passive membrane, and calculated excitability metrics from the resulting traces. Consistent with our expectations of homeostatic compensation mitigating hyperexcitability, we find no significant difference in the maximum amplitude nor AUC observed between 0 and 1 hour. However, we find that at 24 hours there is a significantly higher Amplitude and AUC than at 0 or 1 hour (Figure $3.4 \mathrm{E} \& \mathrm{G}$ ). Over this period, we find that the correlation 
between the observed and expected voltage response is significantly lower at 1 hour than at control and increases to a level not significantly different from either group at 24 hours (Figure 3.4 A). These findings are consistent with the previously noted increase in membrane resistance at 24 hours as a single EPSP protocol was applied; it was not scaled inversely proportionate to membrane resistance. Accounting for the change in resistance we find no significant effect on AUC, although the amplitude remains significantly higher (Figure $3.4 \mathrm{~K} \& \mathrm{I}$ ).

We find the inner quartile range of the correlation calculated across the sweeps for a given sample is significantly lower at 1 hour than control and regresses to an intermediate value at 24 hours (Figure 3.4 B). This indicates that not only does the nonlinearity of a cell's response increase at 1 hour, but the consistency of that response decreases at the same time. Over the same time course, the inner quartile range of the maximum amplitude observed decreased. Together these metrics suggest that at 24 hours cells reach higher, less variable amplitudes presumably due to increased activation of outward currents at these voltages. They depolarize more as well but this appears to be a result of increased membrane resistance rather than insufficient $\mathrm{I}_{\mathrm{A}}$ magnitude per se.

The present study does not examine all aspects of the ganglion. We focused on cell specific properties and therefore cannot comment on changes between cells (e.g. coupling, EPSP properties, stimulation frequency) are altered. This is a promising direction for future study. Based on present data we conclude there is a failure to maintain homeostatic plasticity over a prolonged challenge. Measurements of the synaptic current or spiking statistics could invalidate this conclusion, suggesting instead that multiple compensatory regimes, relying on changes in stimulation or spike initiation properties. 


\section{Conclusion}

In this study we aimed to establish whether potassium channel blockade induces transcriptional changes or if compensation is mediated purely post translationally and if compensatory changes are stable for 24 hours. We find evidence of rapid ( $<=1$ hour) changes in transcript abundance and transcript correlations. The abundance changes and some of the correlational changes regress at 24 hours becoming indistinguishable from baseline. We also report changes in $I_{A}$ and membrane resistance which do not manifest at 1 hour, suggesting that despite a non-significant difference in excitability metrics, apart from correlation relative to expected output, the cell has not reached steady state at this time. Finally, we find an increase in excitability at 24 hours. This is suggestive that these networks may be unable to compensate to a perturbation of this severity over longer timescales or that cell specific changes are insufficient to maintain excitability over this duration, suggesting that changes in synaptic or network properties may be needed to restore baseline activity over the long run. 
Figure Legends, Tables, Extended Data, and Multimedia

Figure 3.1 Increased Activity Alters and Strengthens Correlations
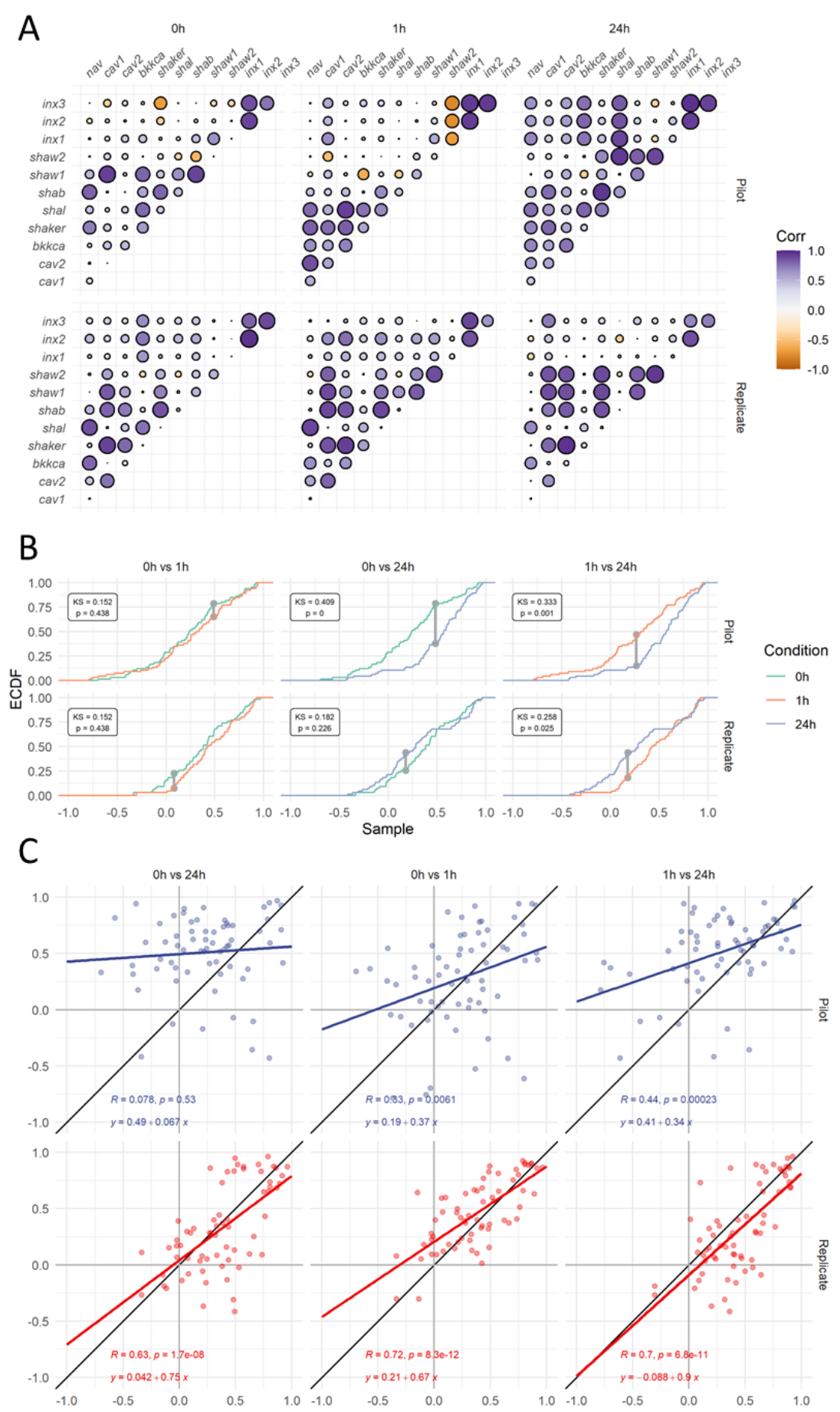
A. Pearson correlation coefficients during incubation TEA for 0 hours (control), 1 hour, or 24 hours. Certain strong positive correlations (e.g. inx1 vs inx2) are visible across time points and replications. Apparent strengthening of select correlations over time, but without uniformity between trials.

B. Changes in $\mathrm{R}$ shown by plotting a given comparison as the correlation at a later time as a function of its correlation at an earlier time. Correlation of correlations and OLS regression formula are shown. Replicate trial shows higher correlations or correlations and slopes closer to unity suggesting less change in correlation strength across time points.

C. The distribution of correlation coefficients becomes significantly more positive after a 24-hour incubation in TEA relative to control ( 0 hours) and 1 hour incubation in the pilot while the distribution at 1 hour is significantly more positive than 24 hours in the replicate (Kolmogorov-Smirnov test). In both trials the ECDFs converge at higher $(\mathrm{R}>=0.7)$ correlation. 
Figure 3.2 Limited, Rapid changes in channel and gap junction mRNA abundances

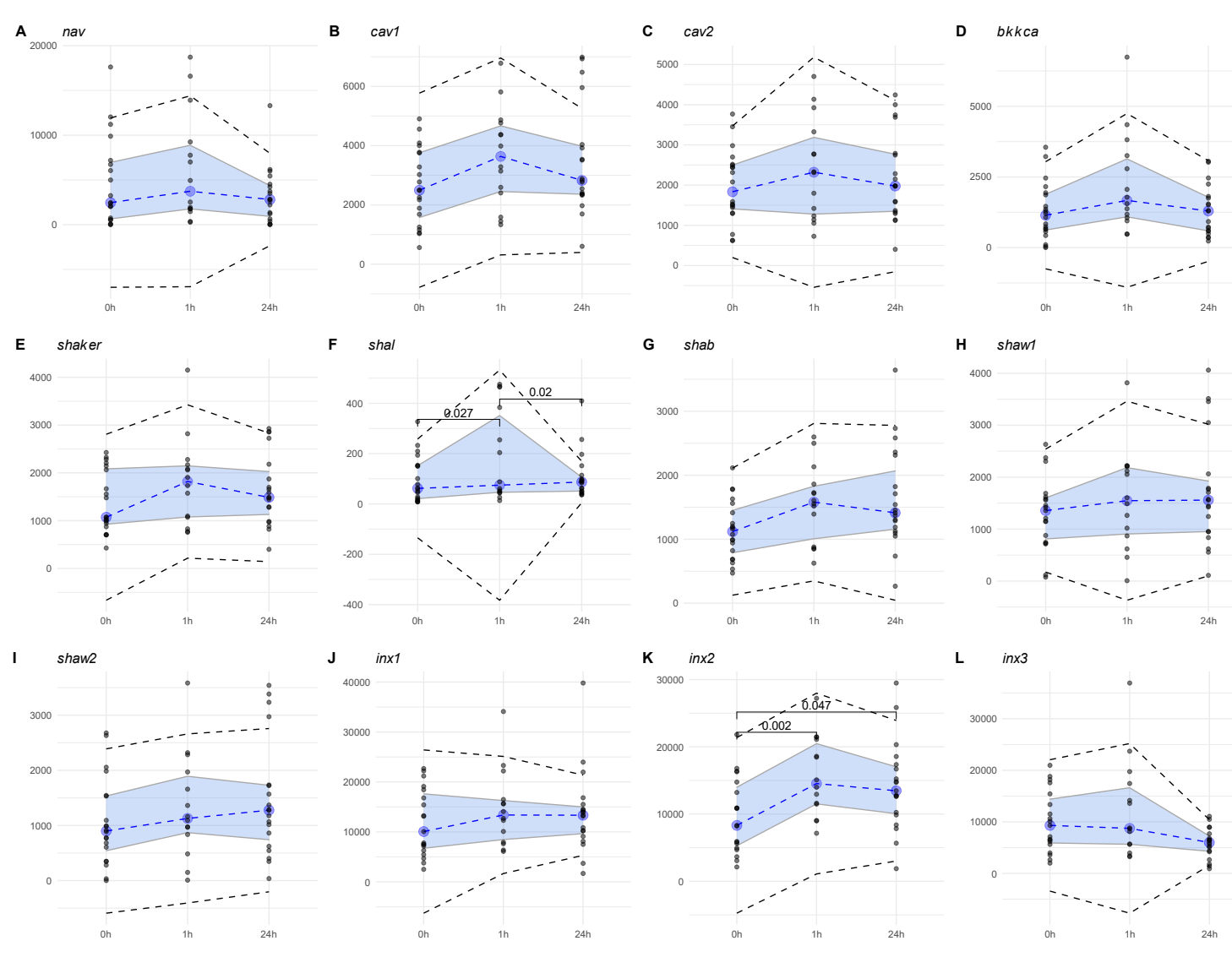

Expression of the voltage activated sodium channel (A.), voltage activated calcium channels (B \& C.), large conductance calcium activated potassium channel (D.), and delayed rectifier potassium channels $(\mathbf{G}, \mathbf{H}, \boldsymbol{\&} \mathbf{I}$.) are not significantly changed thought the treatment. Mean A-type potassium channel encoding shal (F) is elevated at 1 hour but shaker $(E)$ is not significantly affected. Within the gap junction encoding transcripts, inx2 (K) is increased at 1 hour and remains elevated, but inx $(\mathrm{J})$ and in $\times 3(\mathbf{L})$ are unaffected. 
Figure 3.3 Excitability Metrics Are Inconsistent with Homeostasis at 24.

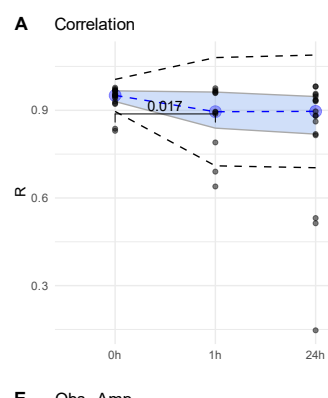

B Correlation IQR

C Baseline

D Baseline IQR

E Obs. Amp.
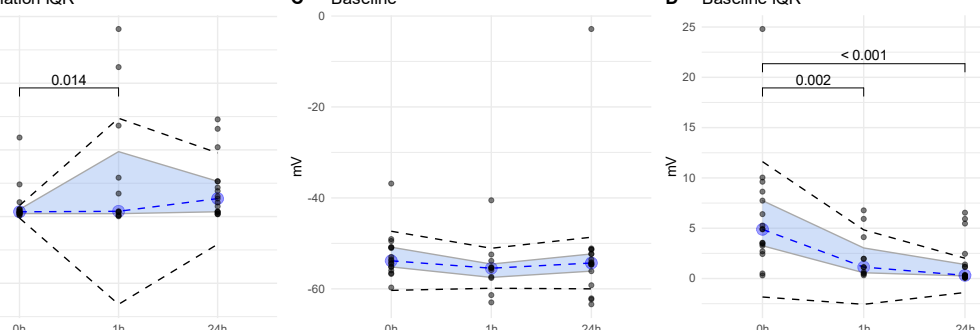

15

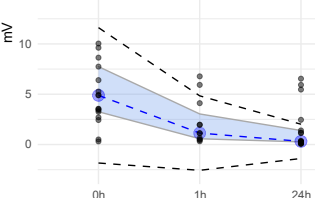

G Obs. AUC

H Obs. AUC IQR

F Obs. Amp. IQR

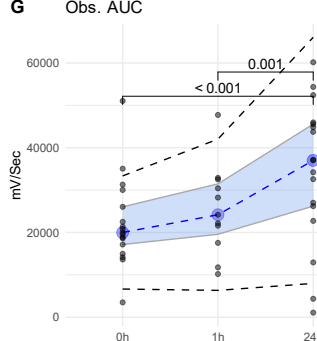

30000

AUC IQR

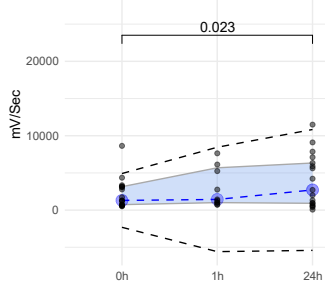

L Diff. AUC IQR
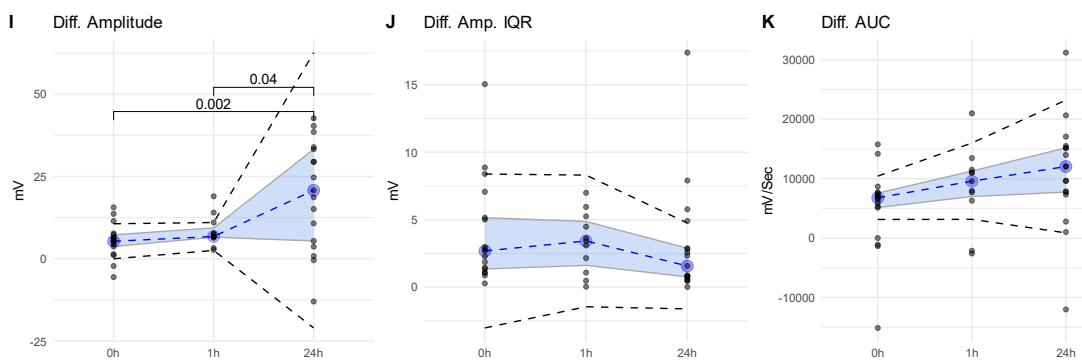

J Diff. Amp. IQR
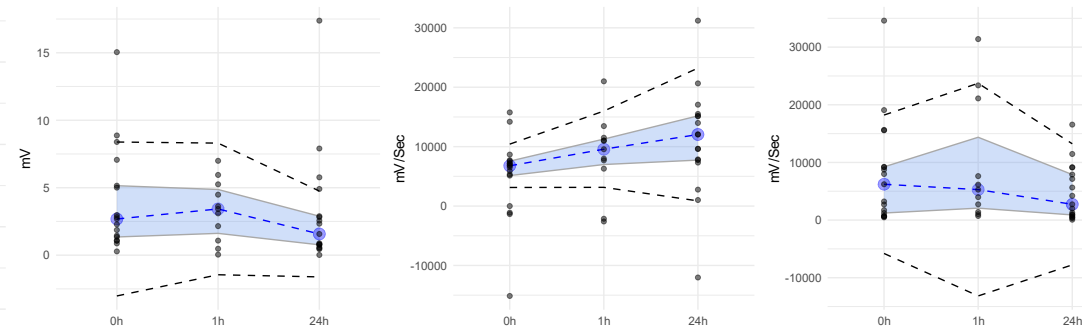

M Sim. Amp

N Sim. AUC
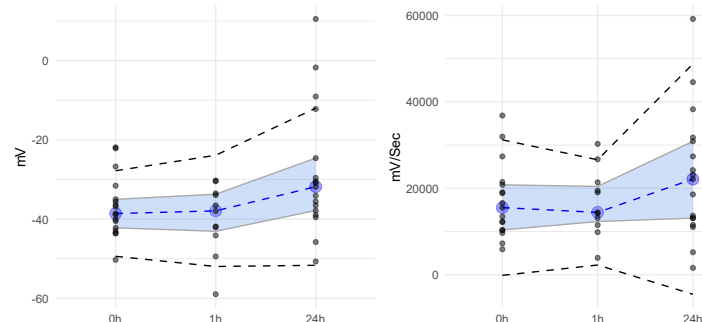

A. Correlation between simulated and observed membrane response does not recover to its baseline level. B. The variation in correlation for a given observation increases coincident with the decrease in correlation IQR. C \& D. Median baseline voltage is unaffected but the variation in baseline voltage decreases. E \& F. Observed amplitude durign stimulation rises at 24 hours, coincidnent with a decrease in the IQR of the amplitude. G \& H. AUC increases at 24 hours with AUC IQR increaseing at 24 hours 
relative to 0 hours. I \& J. The difference between obseved and simulated amplitude increases at 24 hours without a change in IQR. K \& L. The difference between obsrved and simulated AUC and the IQR thereof do not change significantly. $\mathbf{M} \& \mathbf{N}$. The simulated amplitude and simulated AUC do not change significantly. 

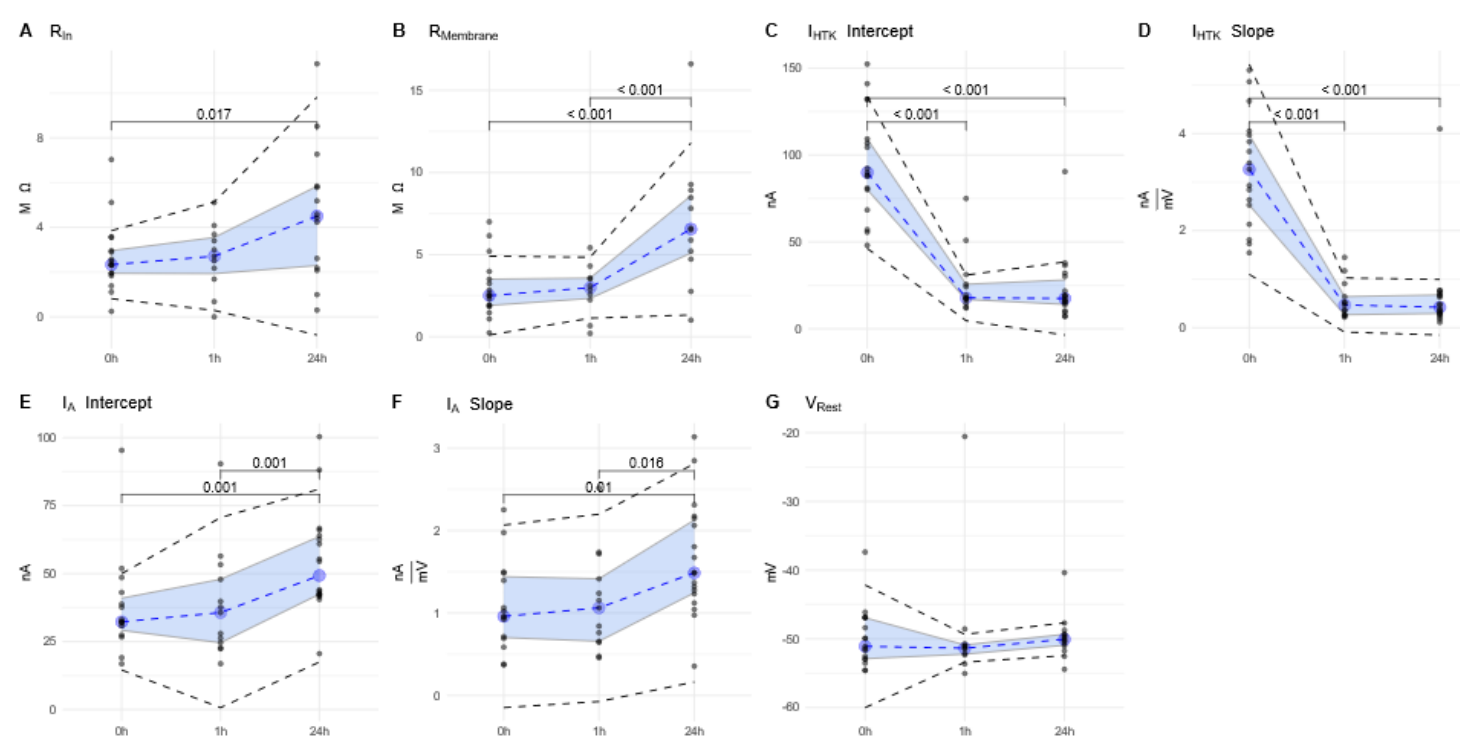

\begin{abstract}
A. Input resistance is significantly higher a t 24 hours relative to 0 hours. B. Membrane resistance increases significantly at 1 and 24 hours. C \& D. I HTK $_{\text {slope of activation and }}$ intercept are reduced through the full duration of the experiment. E \& F. $\mathrm{I}_{\mathrm{A}}$ slope of activation and intercept increase significantly different at 24 hours relative to previous time points. G. Resting membrane potential is not significantly affected over the experimental time course.
\end{abstract}


Table 3.1: Comparison of the Number Strong Correlations Across

\section{Replicates}

\begin{tabular}{llll}
\hline $\mathbf{R}>\mathbf{= 0 . 7}$ & $\mathbf{0 h}$ & $\mathbf{1 h}$ & $\mathbf{2 4 h}$ \\
\hline Both & 6 & 5 & 8 \\
\hline Pilot & 4 & 6 & 12 \\
\hline Replicate & 9 & 10 & 9 \\
\hline Neither & 47 & 45 & 37 \\
\hline
\end{tabular}


Table 3.2: . Subset of Strong Correlations Observed Across Replications.

\begin{tabular}{|c|c|c|c|c|c|c|c|}
\hline & & Pilot Oh & Replicate Oh & Pilot 1h & Replicate 1h & Pilot 24h & Replicate $24 \mathrm{~h}$ \\
\hline bkkca & inx1 & 0.435 & 0.626 & 0.181 & 0.395 & 0.748 & 0.096 \\
\hline bkkca & inx2 & 0.159 & 0.736 & 0.228 & 0.406 & 0.796 & 0.090 \\
\hline bkkca & $\operatorname{in} \times 3$ & -0.174 & 0.665 & 0.374 & 0.373 & 0.764 & 0.252 \\
\hline bkkca & shab & 0.566 & 0.272 & 0.120 & 0.390 & 0.274 & 0.283 \\
\hline bkkca & shaker & 0.612 & 0.066 & 0.558 & 0.591 & 0.361 & 0.297 \\
\hline bkkca & shal & 0.786 & 0.762 & 0.757 & 0.513 & 0.803 & 0.430 \\
\hline bkkca & shaw1 & 0.802 & -0.092 & -0.612 & 0.181 & -0.430 & 0.152 \\
\hline bkkca & shaw2 & -0.057 & -0.335 & -0.092 & 0.079 & -0.127 & 0.112 \\
\hline cav1 & bkkca & 0.441 & 0.010 & 0.578 & 0.398 & 0.572 & 0.171 \\
\hline cav1 & cav2 & -0.014 & 0.750 & 0.569 & 0.802 & 0.568 & 0.647 \\
\hline cav1 & in $x 1$ & 0.269 & 0.308 & 0.669 & 0.438 & 0.408 & 0.398 \\
\hline cav1 & inx2 & 0.221 & 0.356 & 0.487 & 0.667 & 0.515 & 0.443 \\
\hline cav1 & in $x 3$ & -0.412 & 0.381 & 0.545 & 0.102 & 0.315 & 0.730 \\
\hline cav1 & shab & 0.110 & 0.806 & 0.382 & 0.893 & 0.780 & 0.736 \\
\hline cav1 & shaker & 0.404 & 0.915 & 0.781 & 0.859 & 0.793 & 0.793 \\
\hline cav1 & shal & 0.235 & -0.114 & 0.540 & 0.051 & 0.622 & -0.209 \\
\hline cav1 & shaw1 & 0.922 & 0.839 & 0.443 & 0.886 & 0.416 & 0.874 \\
\hline cav1 & shaw2 & 0.367 & 0.572 & -0.527 & 0.811 & 0.316 & 0.855 \\
\hline cav2 & bkkca & 0.476 & 0.281 & 0.706 & 0.634 & 0.747 & 0.282 \\
\hline cav2 & inx1 & 0.398 & 0.207 & 0.060 & 0.500 & 0.537 & -0.057 \\
\hline cav2 & inx2 & -0.124 & 0.438 & 0.076 & 0.767 & 0.601 & 0.336 \\
\hline cav2 & inx3 & 0.296 & 0.319 & 0.258 & 0.216 & 0.593 & 0.263 \\
\hline cav2 & shab & 0.039 & 0.729 & 0.461 & 0.847 & 0.529 & 0.830 \\
\hline cav2 & shaker & 0.245 & 0.790 & 0.820 & 0.923 & 0.543 & 0.962 \\
\hline cav2 & shal & 0.364 & 0.427 & 0.921 & 0.345 & 0.515 & -0.132 \\
\hline cav2 & shaw1 & 0.194 & 0.574 & -0.183 & 0.655 & 0.523 & 0.871 \\
\hline cav2 & shaw2 & 0.318 & 0.277 & 0.083 & 0.371 & 0.164 & 0.860 \\
\hline inx 1 & inx2 & 0.907 & 0.964 & 0.926 & 0.864 & 0.925 & 0.871 \\
\hline
\end{tabular}




\begin{tabular}{|c|c|c|c|c|c|c|c|}
\hline & & Pilot Oh & Replicate Oh & Pilot 1h & Replicate 1h & Pilot 24h & Replicate $24 \mathrm{~h}$ \\
\hline $\operatorname{inx} 1$ & $\operatorname{in} \times 3$ & 0.873 & 0.809 & 0.941 & 0.905 & 0.968 & 0.693 \\
\hline inx2 & in $x 3$ & 0.754 & 0.893 & 0.941 & 0.630 & 0.912 & 0.721 \\
\hline nav & bkkca & 0.235 & 0.793 & 0.660 & 0.659 & 0.694 & 0.651 \\
\hline nav & cav1 & 0.330 & 0.082 & 0.527 & 0.120 & 0.423 & -0.080 \\
\hline nav & cav2 & 0.123 & 0.436 & 0.869 & 0.559 & 0.624 & 0.043 \\
\hline nav & inx 1 & 0.112 & 0.214 & -0.067 & 0.201 & 0.693 & -0.367 \\
\hline nav & inx2 & -0.298 & 0.483 & -0.063 & 0.318 & 0.599 & -0.308 \\
\hline nav & inx3 & -0.024 & 0.336 & 0.070 & 0.128 & 0.654 & 0.085 \\
\hline nav & shab & 0.808 & 0.491 & 0.436 & 0.154 & 0.592 & 0.132 \\
\hline nav & shaker & 0.717 & 0.245 & 0.821 & 0.238 & 0.696 & 0.051 \\
\hline nav & shal & 0.421 & 0.870 & 0.786 & 0.903 & 0.768 & 0.683 \\
\hline nav & shaw1 & 0.482 & -0.021 & -0.261 & 0.225 & -0.072 & 0.068 \\
\hline nav & shaw2 & 0.074 & -0.134 & 0.071 & -0.303 & 0.334 & -0.188 \\
\hline shab & inx1 & 0.453 & 0.420 & -0.014 & 0.328 & 0.384 & 0.157 \\
\hline shab & inx2 & -0.201 & 0.532 & -0.138 & 0.567 & 0.362 & -0.024 \\
\hline shab & in $x 3$ & -0.026 & 0.425 & -0.017 & 0.013 & 0.254 & 0.405 \\
\hline shab & shaw1 & 0.908 & 0.488 & 0.505 & 0.828 & 0.708 & 0.834 \\
\hline shab & shaw2 & -0.571 & 0.429 & 0.363 & 0.685 & 0.816 & 0.829 \\
\hline shaker & in $x 1$ & -0.168 & 0.203 & 0.290 & 0.464 & 0.449 & 0.080 \\
\hline shaker & inx2 & -0.417 & 0.384 & 0.255 & 0.658 & 0.392 & 0.369 \\
\hline shaker & inx3 & -0.695 & 0.332 & 0.338 & 0.272 & 0.331 & 0.347 \\
\hline shaker & shab & 0.801 & 0.860 & 0.670 & 0.895 & 0.949 & 0.860 \\
\hline shaker & shal & 0.394 & 0.023 & 0.681 & 0.091 & 0.736 & -0.140 \\
\hline shaker & shaw1 & 0.424 & 0.704 & 0.258 & 0.753 & 0.562 & 0.892 \\
\hline shaker & shaw2 & 0.308 & 0.519 & 0.017 & 0.597 & 0.690 & 0.893 \\
\hline shal & inx1 & 0.217 & 0.238 & 0.048 & 0.420 & 0.905 & -0.159 \\
\hline shal & inx2 & 0.037 & 0.493 & 0.032 & 0.362 & 0.858 & -0.415 \\
\hline shal & in $x 3$ & -0.047 & 0.383 & 0.254 & 0.399 & 0.835 & -0.014 \\
\hline shal & shab & 0.464 & 0.184 & 0.415 & 0.147 & 0.619 & 0.036 \\
\hline shal & shaw1 & 0.658 & -0.147 & -0.432 & 0.641 & -0.103 & -0.087 \\
\hline
\end{tabular}




\begin{tabular}{lllccccc}
\hline & & Pilot 0h & Replicate 0h & Pilot 1h & Replicate 1h & Pilot 24h & Replicate 24h \\
\hline shal & shaw2 & -0.385 & -0.336 & -0.046 & -0.304 & $\mathbf{0 . 9 4 0}$ & -0.268 \\
\hline shaw1 & inx1 & 0.650 & 0.077 & 0.537 & 0.468 & -0.357 & 0.058 \\
\hline shaw1 & inx2 & 0.207 & 0.101 & 0.341 & 0.599 & -0.172 & 0.283 \\
\hline shaw1 & inx3 & -0.339 & 0.130 & 0.222 & 0.182 & -0.417 & 0.323 \\
\hline shaw1 & shaw2 & 0.045 & 0.497 & 0.308 & $\mathbf{0 . 8 7 8}$ & $\mathbf{0 . 8 9 8}$ & $\mathbf{0 . 9 4 8}$ \\
\hline shaw2 & inx1 & -0.034 & -0.023 & -0.696 & 0.279 & 0.373 & 0.171 \\
\hline shaw2 & inx2 & -0.070 & -0.009 & -0.756 & 0.251 & 0.417 & 0.389 \\
\hline shaw2 & inx3 & -0.368 & -0.020 & -0.786 & 0.094 & 0.175 & 0.219 \\
\hline
\end{tabular}


Table 3.3: Bivariate transcript abundance relationships.

\begin{tabular}{|c|c|c|c|c|c|c|c|c|}
\hline & & ANCOVA padj & $\mathbf{p a d j}_{\mathrm{O}} \mathrm{Oh}$ & $p_{\text {adj }} 1 \mathrm{~h}$ & $p_{a d j} 24 h$ & R Oh & R 1h & R 24h \\
\hline nav & cav1 & 0.980 & 0.857 & 0.736 & 0.876 & 0.082 & 0.120 & -0.080 \\
\hline nav & cav2 & 0.960 & 0.188 & 0.072 & 0.897 & 0.436 & 0.559 & 0.043 \\
\hline nav & bkkca & 0.980 & 0.001 & 0.025 & 0.011 & 0.793 & 0.659 & 0.651 \\
\hline nav & shaker & 0.980 & 0.481 & 0.533 & 0.885 & 0.245 & 0.238 & 0.051 \\
\hline nav & shal & 0.099 & 0.000 & 0.000 & 0.007 & 0.870 & 0.903 & 0.683 \\
\hline nav & shab & 0.960 & 0.129 & 0.676 & 0.780 & 0.491 & 0.154 & 0.132 \\
\hline nav & shaw1 & 0.975 & 0.972 & 0.549 & 0.876 & -0.021 & 0.225 & 0.068 \\
\hline nav & shaw2 & 0.960 & 0.759 & 0.419 & 0.672 & -0.134 & -0.303 & -0.188 \\
\hline nav & $\operatorname{in} x 1$ & 0.910 & 0.563 & 0.600 & 0.369 & 0.214 & 0.201 & -0.367 \\
\hline nav & inx2 & 0.561 & 0.151 & 0.392 & 0.458 & 0.483 & 0.318 & -0.308 \\
\hline nav & $\operatorname{inx} 3$ & 0.960 & 0.322 & 0.736 & 0.872 & 0.336 & 0.128 & 0.085 \\
\hline cav1 & cav2 & 0.960 & 0.001 & 0.001 & 0.016 & 0.750 & 0.802 & 0.647 \\
\hline cav1 & bkkca & 0.897 & 0.972 & 0.245 & 0.779 & 0.010 & 0.398 & 0.171 \\
\hline cav1 & shaker & 0.960 & 0.000 & 0.000 & 0.001 & 0.915 & 0.859 & 0.793 \\
\hline cav1 & shal & 0.975 & 0.759 & 0.876 & 0.698 & -0.114 & 0.051 & -0.209 \\
\hline cav1 & shab & 0.960 & 0.000 & 0.000 & 0.003 & 0.806 & 0.893 & 0.736 \\
\hline cav1 & shaw1 & 0.490 & 0.000 & 0.000 & 0.000 & 0.839 & 0.886 & 0.874 \\
\hline cav1 & shaw2 & 0.521 & 0.038 & 0.001 & 0.000 & 0.572 & 0.811 & 0.855 \\
\hline cav1 & $\operatorname{in} x 1$ & 0.980 & 0.315 & 0.206 & 0.369 & 0.308 & 0.438 & 0.398 \\
\hline cav1 & inx2 & 0.960 & 0.246 & 0.019 & 0.298 & 0.356 & 0.667 & 0.443 \\
\hline cav1 & $\operatorname{in} x 3$ & 0.910 & 0.194 & 0.777 & 0.003 & 0.381 & 0.102 & 0.730 \\
\hline cav2 & bkkca & 0.490 & 0.414 & 0.026 & 0.458 & 0.281 & 0.634 & 0.282 \\
\hline cav2 & shaker & 0.939 & 0.000 & 0.000 & 0.000 & 0.790 & 0.923 & 0.962 \\
\hline cav2 & shal & 0.521 & 0.151 & 0.349 & 0.779 & 0.427 & 0.345 & -0.132 \\
\hline cav2 & shab & 0.960 & 0.002 & 0.000 & 0.000 & 0.729 & 0.847 & 0.830 \\
\hline cav2 & shaw1 & 0.499 & 0.079 & 0.022 & 0.000 & 0.574 & 0.655 & 0.871 \\
\hline cav2 & shaw2 & 0.183 & 0.414 & 0.266 & 0.000 & 0.277 & 0.371 & 0.860 \\
\hline
\end{tabular}




\begin{tabular}{|c|c|c|c|c|c|c|c|c|}
\hline & & ANCOVA padj & Padj $0 \mathrm{~h}$ & $p_{\text {adj }} 1 \mathrm{~h}$ & Padj 24h & R Oh & R 1h & R $24 \mathrm{~h}$ \\
\hline cav2 & $\operatorname{in} x 1$ & 0.789 & 0.551 & 0.132 & 0.885 & 0.207 & 0.500 & -0.057 \\
\hline cav2 & inx2 & 0.561 & 0.167 & 0.002 & 0.369 & 0.438 & 0.767 & 0.336 \\
\hline cav2 & $\operatorname{in} x 3$ & 0.960 & 0.322 & 0.549 & 0.458 & 0.319 & 0.216 & 0.263 \\
\hline bkkca & shaker & 0.960 & 0.873 & 0.052 & 0.435 & 0.066 & 0.591 & 0.297 \\
\hline bkkca & shal & 0.641 & 0.001 & 0.134 & 0.245 & 0.762 & 0.513 & 0.430 \\
\hline bkkca & shab & 0.980 & 0.414 & 0.249 & 0.459 & 0.272 & 0.390 & 0.283 \\
\hline$b k k c a$ & shaw1 & 0.960 & 0.857 & 0.623 & 0.779 & -0.092 & 0.181 & 0.152 \\
\hline bkkca & shaw2 & 0.960 & 0.322 & 0.803 & 0.823 & -0.335 & 0.079 & 0.112 \\
\hline bkkca & $\operatorname{in} x 1$ & 0.499 & 0.028 & 0.259 & 0.872 & 0.626 & 0.395 & 0.096 \\
\hline$b k k c a$ & $\operatorname{in} x 2$ & 0.561 & 0.005 & 0.249 & 0.872 & 0.736 & 0.406 & 0.090 \\
\hline bkkca & $\operatorname{in} x 3$ & 0.630 & 0.015 & 0.283 & 0.492 & 0.665 & 0.373 & 0.252 \\
\hline shaker & shal & 0.980 & 0.972 & 0.803 & 0.779 & 0.023 & 0.091 & -0.140 \\
\hline shaker & shab & 0.960 & 0.000 & 0.000 & 0.000 & 0.860 & 0.895 & 0.860 \\
\hline shaker & shaw1 & 0.319 & 0.007 & 0.005 & 0.000 & 0.704 & 0.753 & 0.892 \\
\hline shaker & shaw2 & 0.191 & 0.075 & 0.042 & 0.000 & 0.519 & 0.597 & 0.893 \\
\hline shaker & $\operatorname{in} x 1$ & 0.910 & 0.538 & 0.196 & 0.876 & 0.203 & 0.464 & 0.080 \\
\hline shaker & inx2 & 0.747 & 0.199 & 0.025 & 0.310 & 0.384 & 0.658 & 0.369 \\
\hline shaker & $\operatorname{in} x 3$ & 0.960 & 0.271 & 0.467 & 0.304 & 0.332 & 0.272 & 0.347 \\
\hline shal & shab & 0.988 & 0.567 & 0.702 & 0.916 & 0.184 & 0.147 & 0.036 \\
\hline shal & shaw1 & 0.490 & 0.745 & 0.048 & 0.872 & -0.147 & 0.641 & -0.087 \\
\hline shal & shaw2 & 0.960 & 0.300 & 0.439 & 0.459 & -0.336 & -0.304 & -0.268 \\
\hline shal & $\operatorname{in} x 1$ & 0.960 & 0.469 & 0.266 & 0.779 & 0.238 & 0.420 & -0.159 \\
\hline shal & $\operatorname{in} x 2$ & 0.490 & 0.113 & 0.349 & 0.304 & 0.493 & 0.362 & -0.415 \\
\hline shal & $\operatorname{in} x 3$ & 0.960 & 0.199 & 0.284 & 0.957 & 0.383 & 0.399 & -0.014 \\
\hline shab & shaw1 & 0.490 & 0.129 & 0.000 & 0.000 & 0.488 & 0.828 & 0.834 \\
\hline shab & shaw2 & 0.490 & 0.151 & 0.014 & 0.000 & 0.429 & 0.685 & 0.829 \\
\hline shab & $\operatorname{in} x 1$ & 0.910 & 0.151 & 0.350 & 0.779 & 0.420 & 0.328 & 0.157 \\
\hline shab & inx2 & 0.521 & 0.066 & 0.054 & 0.943 & 0.532 & 0.567 & -0.024 \\
\hline shab & $\operatorname{in} x 3$ & 0.866 & 0.151 & 0.963 & 0.245 & 0.425 & 0.013 & 0.405 \\
\hline shaw1 & shaw2 & 0.897 & 0.151 & 0.000 & 0.000 & 0.497 & 0.878 & 0.948 \\
\hline
\end{tabular}




\begin{tabular}{llccccccc}
\hline & & ANCOVA padj & padj $\mathbf{h}$ & padj $\mathbf{h}$ & padj $\mathbf{2 4}$ & $\mathbf{R}$ Oh & $\mathbf{R} \mathbf{1 h}$ & $\mathbf{R} \mathbf{2 4 h}$ \\
\hline shaw1 & inx1 & 0.960 & 0.870 & 0.195 & 0.885 & 0.077 & 0.468 & 0.058 \\
\hline shaw1 & inx2 & 0.767 & 0.849 & $\mathbf{0 . 0 4 8}$ & 0.459 & 0.101 & $\mathbf{0 . 5 9 9}$ & 0.283 \\
\hline shaw1 & inx3 & 0.980 & 0.759 & 0.629 & 0.369 & 0.130 & 0.182 & 0.323 \\
\hline shaw2 & inx1 & 0.960 & 0.972 & 0.459 & 0.769 & -0.023 & 0.279 & 0.171 \\
\hline shaw2 & inx2 & 0.960 & 0.972 & 0.485 & 0.298 & -0.009 & 0.251 & 0.389 \\
\hline shaw2 & inx3 & 0.980 & 0.972 & 0.797 & 0.546 & -0.020 & 0.094 & 0.219 \\
\hline inx1 & inx2 & 0.844 & $\mathbf{0 . 0 0 0}$ & $\mathbf{0 . 0 0 0}$ & $\mathbf{0 . 0 0 0}$ & $\mathbf{0 . 9 6 4}$ & $\mathbf{0 . 8 6 4}$ & $\mathbf{0 . 8 7 1}$ \\
\hline inx1 & inx3 & 0.183 & $\mathbf{0 . 0 0 0}$ & $\mathbf{0 . 0 0 0}$ & $\mathbf{0 . 0 0 7}$ & $\mathbf{0 . 8 0 9}$ & $\mathbf{0 . 9 0 5}$ & $\mathbf{0 . 6 9 3}$ \\
\hline inx2 & inx3 & 0.490 & $\mathbf{0 . 0 0 0}$ & $\mathbf{0 . 0 3 6}$ & $\mathbf{0 . 0 0 3}$ & $\mathbf{0 . 8 9 3}$ & $\mathbf{0 . 6 3 0}$ & $\mathbf{0 . 7 2 1}$ \\
\hline
\end{tabular}


Table 3.4: Summary of statistical effects of treatment duration on measured properties.

\begin{tabular}{|c|c|c|c|c|c|c|c|c|c|c|c|}
\hline \multirow{3}{*}{$\begin{array}{l}\text { Family } \\
\text { Membrane } \\
\text { Resistance }\end{array}$} & \multirow[b]{2}{*}{ Input resistance $\left(R_{\mathrm{in}}, \mathrm{r} 11\right)$} & \multirow{2}{*}{$\begin{array}{r}p \\
0.0144\end{array}$} & \multirow{2}{*}{$\begin{array}{r}\text { ep } \\
0.020\end{array}$} & \multicolumn{2}{|l|}{$\begin{array}{l}\text { FDR } \\
\text { ep adj }\end{array}$} & \multicolumn{2}{|c|}{$\begin{array}{l}\text { Group and } \\
\text { Estimate } 0 \mathrm{~h}\end{array}$} & \multicolumn{2}{|c|}{$\begin{array}{l}\text { Group and } \\
\text { Estimate } 1 \mathrm{~h}\end{array}$} & \multicolumn{2}{|c|}{$\begin{array}{r}\text { Group and } \\
\text { Estimate } 24 \mathrm{~h}\end{array}$} \\
\hline & & & & 0.0370 & * & A & 2.4789 & $A B$ & 2.6023 & B & 3.6702 \\
\hline & Membrane resistance $\left(\mathbf{R}_{\text {mem }}, \mathrm{r} 1\right)$ & 0.0000 & 0.000 & 0.0000 & $\star \star \star *$ & $\mathbf{A}$ & 2.6238 & A & 2.8150 & B & 5.8282 \\
\hline $\begin{array}{l}\text { Resting } \\
\text { Voltage }\end{array}$ & $V_{\text {rest }}$ & 0.0064 & 0.005 & 0.0116 & * & AB & -50.6279 & A & -51.0803 & B & -50.1705 \\
\hline \multirow{8}{*}{$\begin{array}{l}\text { Outward } \\
\text { Currents }\end{array}$} & Intk Intercept, Transient & 0.0000 & 0.000 & 0.0000 & *** & B & 92.9582 & A & 19.6754 & A & 14.4563 \\
\hline & Intk Slope, Transient & 0.0000 & 0.000 & 0.0000 & $* * *$ & B & 3.2946 & A & 0.4182 & A & 0.4396 \\
\hline & Ihtk Intercept, Sustained & 0.0000 & 0.000 & 0.0000 & $\star \star \star *$ & B & 74.8268 & A & 16.1830 & A & 11.1426 \\
\hline & Ihtk Slope, Sustained & 0.0000 & 0.000 & 0.0000 & $\star \star * *$ & B & 2.4315 & A & 0.3487 & A & 0.3642 \\
\hline & $I_{A}$ Intercept, Transient & 0.0002 & 0.001 & 0.0026 & ** & A & 33.8287 & A & 32.7595 & B & 51.0611 \\
\hline & $\mathrm{I}_{\mathrm{A}}$ Slope, Transient & 0.0044 & 0.001 & 0.0026 & ** & A & 1.0053 & A & 0.9795 & B & 1.5488 \\
\hline & $I_{A}$ Intercept, Sustained & 0.0001 & 0.000 & 0.0000 & $* * *$ & B & 16.6296 & A & 5.9300 & B & 14.8244 \\
\hline & $I_{A}$ Slope, Sustained & 0.0000 & 0.000 & 0.0000 & *** & B & 0.5932 & C & 0.1848 & A & 0.3750 \\
\hline \multirow{4}{*}{ Excitability } & Correlation (Current, Voltage) & 0.0163 & 0.009 & 0.0185 & * & B & 0.9541 & A & 0.8800 & AB & 0.9089 \\
\hline & Min. Voltage Observed & 0.3678 & 0.395 & 0.4567 & & & -53.7811 & & -55.0791 & & -53.3765 \\
\hline & Max Amplitude Observed & 0.0000 & 0.000 & 0.0000 & $\star * *$ & $\mathbf{A}$ & -29.4474 & A & -30.4871 & B & -1.6797 \\
\hline & Max Amplitude Simulated & 0.2627 & 0.285 & 0.3636 & & & -37.9020 & & -38.7625 & & -34.6902 \\
\hline
\end{tabular}




\begin{tabular}{|c|c|c|c|c|c|c|c|c|c|c|c|}
\hline \multicolumn{2}{|l|}{ Family } & $\mathbf{p}$ & \multirow{2}{*}{$\begin{array}{r}\text { ep } \\
0.000\end{array}$} & \multicolumn{2}{|l|}{$\begin{array}{l}\text { FDR } \\
\text { epadj }\end{array}$} & \multicolumn{2}{|c|}{$\begin{array}{l}\text { Group and } \\
\text { Estimate } 0 \mathrm{~h}\end{array}$} & \multicolumn{2}{|c|}{$\begin{array}{l}\text { Group and } \\
\text { Estimate 1h }\end{array}$} & \multicolumn{2}{|c|}{$\begin{array}{r}\text { Group and } \\
\text { Estimate } 24 \mathrm{~h}\end{array}$} \\
\hline & AUC Observed & 0.0001 & & 0.0000 & *** & A & $\begin{array}{l}21,073.4 \\
583\end{array}$ & A & $\begin{array}{l}21,299.9 \\
011\end{array}$ & B & $\begin{array}{l}38,574.38 \\
63\end{array}$ \\
\hline & AUC Simulated & 0.1258 & 0.135 & 0.1850 & & & $\begin{array}{l}15,099.2 \\
597\end{array}$ & & $\begin{array}{l}13,187.7 \\
660\end{array}$ & & $\begin{array}{l}20,690.60 \\
55\end{array}$ \\
\hline & Max Amplitude Delta & 0.0017 & 0.001 & 0.0026 & ** & $\mathbf{A}$ & 5.3127 & A & 7.0121 & B & 20.9107 \\
\hline & AUC Delta & 0.0453 & 0.053 & 0.0784 & . & & $\begin{array}{l}6,950.01 \\
97\end{array}$ & & $\begin{array}{l}9,576.15 \\
20\end{array}$ & & $\begin{array}{l}11,282.15 \\
97\end{array}$ \\
\hline & Correlation IQR & 0.0183 & 0.007 & 0.0152 & * & A & 0.0065 & B & 0.0597 & $A B$ & 0.0225 \\
\hline & Min Voltage Observed IQR & 0.0000 & 0.000 & 0.0000 & $* * *$ & B & 4.8192 & A & 1.0823 & A & 0.6822 \\
\hline & Max Amplitude Observed IQR & 0.0042 & 0.003 & 0.0074 & ** & B & 4.6443 & B & 4.7930 & A & 1.6270 \\
\hline & AUC Observed IQR & 0.0300 & 0.033 & 0.0555 & & & $\begin{array}{l}1,825.34 \\
29\end{array}$ & & $\begin{array}{l}3,192.85 \\
42\end{array}$ & & $\begin{array}{l}4,445.240 \\
3\end{array}$ \\
\hline & Max Amplitude Delta IQR & 0.1521 & 0.158 & 0.2088 & & & 2.5660 & & 3.6296 & & 1.9288 \\
\hline & AUC Delta IQR & 0.8769 & 0.872 & 0.8720 & & & $\begin{array}{l}4,876.68 \\
10\end{array}$ & & $\begin{array}{l}3,927.47 \\
22\end{array}$ & & $\begin{array}{l}4,545.229 \\
7\end{array}$ \\
\hline $\mathrm{Na}+$ Channel & nav & 0.3559 & 0.352 & 0.4201 & & & $\begin{array}{l}3,543.44 \\
03\end{array}$ & & $\begin{array}{l}4,414.72 \\
54\end{array}$ & & $\begin{array}{l}2,656.222 \\
7\end{array}$ \\
\hline \multirow{2}{*}{$\begin{array}{l}\text { Ca2+ } \\
\text { Channel }\end{array}$} & cav1 & 0.0636 & 0.060 & 0.0854 & . & & $\begin{array}{l}2,610.53 \\
81\end{array}$ & & $\begin{array}{l}3,625.03 \\
27\end{array}$ & & $\begin{array}{l}2,650.673 \\
6\end{array}$ \\
\hline & cav2 & 0.3382 & 0.330 & 0.4070 & & & $\begin{array}{l}1,872.13 \\
70\end{array}$ & & $\begin{array}{l}2,402.08 \\
28\end{array}$ & & $\begin{array}{l}2,063.717 \\
1\end{array}$ \\
\hline $\begin{array}{l}\text { Other K+ } \\
\text { Channel }\end{array}$ & bkkca & 0.0432 & 0.042 & 0.0648 & . & & $\begin{array}{l}1,046.90 \\
07\end{array}$ & & $\begin{array}{l}1,932.83 \\
11\end{array}$ & & $\begin{array}{l}1,314.710 \\
2\end{array}$ \\
\hline
\end{tabular}




\begin{tabular}{|c|c|c|c|c|c|c|c|c|c|c|c|}
\hline Family & & p & ep & $\begin{array}{l}\text { FDR } \\
\text { epadj }\end{array}$ & & & $\begin{array}{l}\text { Group and } \\
\text { Estimate Oh }\end{array}$ & & $\begin{array}{l}\text { Group and } \\
\text { Estimate } 1 \mathrm{~h}\end{array}$ & & $\begin{array}{r}\text { Group and } \\
\text { stimate } 24 \mathrm{~h}\end{array}$ \\
\hline \multirow{5}{*}{$\begin{array}{l}\text { Voltage- } \\
\text { dependent K+ } \\
\text { Channel }\end{array}$} & shaker & 0.5341 & 0.550 & 0.5985 & & & $\begin{array}{l}1,384.45 \\
55\end{array}$ & & $\begin{array}{l}1,627.22 \\
15\end{array}$ & & $\begin{array}{l}1,422.429 \\
6\end{array}$ \\
\hline & shal & 0.0124 & 0.013 & 0.0253 & * & A & 84.1001 & B & 187.9169 & A & 75.0182 \\
\hline & shab & 0.0294 & 0.031 & 0.0546 & . & & $\begin{array}{l}1,093.47 \\
68\end{array}$ & & $\begin{array}{l}1,557.87 \\
83\end{array}$ & & $\begin{array}{l}1,519.613 \\
2\end{array}$ \\
\hline & shaw1 & 0.7551 & 0.759 & 0.8024 & & & $\begin{array}{l}1,370.25 \\
25\end{array}$ & & $\begin{array}{l}1,395.72 \\
51\end{array}$ & & $\begin{array}{l}1,239.470 \\
7\end{array}$ \\
\hline & shaw2 & 0.4307 & 0.428 & 0.4799 & & & 879.3227 & & $\begin{array}{l}1,174.25 \\
40\end{array}$ & & $\begin{array}{l}1,001.998 \\
1\end{array}$ \\
\hline \multirow{3}{*}{ Innexin } & $i n \times 1$ & 0.8107 & 0.815 & 0.8376 & & & $\begin{array}{l}11,836.9 \\
957\end{array}$ & & $\begin{array}{l}13,112.0 \\
121\end{array}$ & & $\begin{array}{l}12,266.58 \\
39\end{array}$ \\
\hline & $i n \times 2$ & 0.0018 & 0.000 & 0.0000 & $\star \star \star *$ & $\mathbf{A}$ & $\begin{array}{l}9,021.78 \\
87\end{array}$ & B & $\begin{array}{l}15,609.0 \\
201\end{array}$ & B & $\begin{array}{l}13,200.98 \\
17\end{array}$ \\
\hline & inx3 & 0.0354 & 0.035 & 0.0563 & . & & $\begin{array}{l}10,005.9 \\
595\end{array}$ & & $\begin{array}{l}10,475.8 \\
073\end{array}$ & & $\begin{array}{l}5,787.396 \\
4\end{array}$ \\
\hline
\end{tabular}


Table 3.S1: Multiplex qRT-PCR Primers Used.

\begin{tabular}{|c|c|c|c|}
\hline Target & Forward Primer & Reverse Primer & Probe \\
\hline Nav & TCAACGGGAGGTACCATAAGTG & TCGCTGTTCACCCAAGAGTAG & CGGAGGGATTGAAGCTCAACGCA \\
\hline Cav1 & CCAGGCCTTCTACTGGCTCATT & GCTGGCGATAGTGCTCACTG & TGTGCTCGTCTTCCTCAACACGG \\
\hline Cav2 & ATCCGGCGGACAGTAAAGC & GTTCGGCAGCAACACAAAC & TGGTTCTACTGGTTCGTCATCATACTTGT \\
\hline Bkkca & GCTCAAACTCGGCTTCATTG & CTGCGTGTCTGGAGAAGTTT & AGAATCCCGGCGCTAAACATGACT \\
\hline Shaker & GAGGCTCAGAAGACCAGTCAAC & TGGCGATATCACCGAGCTCAT & CACTCGATGTCTTCGCGGAGGAGAT \\
\hline Shal & GACACCACCTTCACCTCCATTC & GAACCATGTCGCCGTATCCTA & CGGCGTTTTGGTACACCATTGTCAC \\
\hline Shab & GAGCCGGACAGACAGGAAC & TGCGCCTCCTTCTGTAGTC & AAGAACCACGAACACCACATGGGTC \\
\hline Shaw1 & CGCGTCACTCCTCAGGACTT & CCCAGCACCAGGAAGAACAC & TGATACAGACTTTCCGTGCATCCGC \\
\hline Shaw2 & GAACGCCATCAAGCACTATCATC & ATGGCGCCCGACAGCTTAG & TGGCTTGAAGGACGGTCTCACA \\
\hline $\ln \times 1$ & TGGAGCGTCATGATGCATTC & GAGCAGGATGGCAAGGATCAC & TGCTGCCTCTCAACATTCTTAACGAA \\
\hline $\ln \times 2$ & GGCTGTGGTGTCTGGTGTAG & GAGCGTGTCCTTAACAG & CTGCTGTACCGCCTCGCCACTTT \\
\hline $\ln \times 3$ & TGTCGGCCCTAGTGAAAGAG & GGTACCGTGGGATGTAGAACA & TGACGAGATTGTGTACCACGCTTAC \\
\hline
\end{tabular}




\section{Chapter 4}

\section{Molecular profiling of single neurons of known identity in two ganglia from the crab Cancer borealis}

Adam J. Northcutta,b,1, Daniel R. Kick ${ }^{a, 1}$, Adriane G. Otopalikc, Benjamin M. Goetzd, Rayna M. Harris ${ }^{\mathrm{b}, \mathrm{d}, \mathrm{e}, \mathrm{f}}$, Joseph M. Santin ${ }^{\mathrm{a}}$, Hans A. Hofmann ${ }^{\mathrm{b}, \mathrm{d}, \mathrm{ef}, \mathrm{g}}$, Eve Marder, ${ }^{\mathrm{c}, 2}$, and David J. Schulz $z^{\mathrm{a}, \mathrm{b}, 2}$

aDivision of Biological Sciences, University of Missouri-Columbia, Columbia, MO 65211;

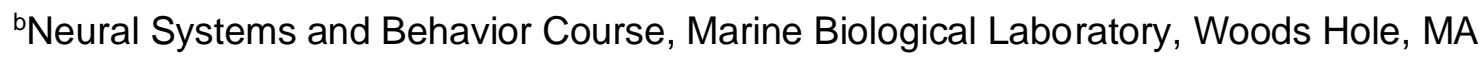
02543;

'Volen Center and Biology Department, Brandeis University, Waltham, MA 02454;

${ }^{\mathrm{d} C e n t e r}$ for Computational Biology and Bioinformatics, The University of Texas at Austin, Austin, TX 78712;

eDepartment of Integrative Biology, The University of Texas at Austin, Austin, TX 78712;

IInstitute for Cellular and Molecular Biology, The University of Texas at Austin, Austin, TX 78712; and

Institute for Neuroscience, The University of Texas at Austin, Austin, TX 78712

Contributed by Eve Marder, October 25, 2019 (sent for review July 5, 2019; reviewed by Gene E. Robinson and Claire Wyart)

${ }^{1}$ A.J.N. and D.R.K. contributed equally to this work.

${ }^{2}$ To whom correspondence may be addressed. Email: marder@brandeis.edu or SchulzD@missouri.edu.

This article contains supporting information online at https://www.pnas.org/lookup/suppl/doi:10.1073/pnas.1911413116/-/DCSupplemental.

First published December 5, 2019. 
Correspondence to:

Dr. David J. Schulz

University of Missouri

Department of Biological Sciences

Columbia, MO, 65211, USA

schulzd@missouri.edu

Correspondence to:

Dr. Eve Marder

Brandeis University

Biology Department and Volen Center

Waltham, MA, 02454, USA

marder@brandeis.edu

Keywords: qPCR RNA-seq stomatogastric expression profiling 


\section{Abstract}

Understanding circuit organization depends on identification of cell types. Recent advances in transcriptional profiling methods have enabled classification of cell types by their gene expression. While exceptionally powerful and high throughput, the ground-truth validation of these methods is difficult: If cell type is unknown, how does one assess whether a given analysis accurately captures neuronal identity? To shed light on the capabilities and limitations of solely using transcriptional profiling for cell-type classification, we performed 2 forms of transcriptional profiling-RNA-seq and quantitative RT-PCR, in single, unambiguously identified neurons from 2 small crustacean neuronal networks: The stomatogastric and cardiac ganglia. We then combined our knowledge of cell type with unbiased clustering analyses and supervised machine learning to determine how accurately functionally defined neuron types can be classified by expression profile alone. The results demonstrate that expression profile is able to capture neuronal identity most accurately when combined with multimodal information that allows for post hoc grouping, so analysis can proceed from a supervised perspective. Solely unsupervised clustering can lead to misidentification and an inability to distinguish between 2 or more cell types. Therefore, this study supports the general utility of cell identification by transcriptional profiling, but adds a caution: It is difficult or impossible to know under what conditions transcriptional profiling alone is capable of assigning cell identity. Only by combining multiple modalities of information such as physiology, 
morphology, or innervation target can neuronal identity be unambiguously determined.

\section{Significance}

Single-cell transcriptional profiling has become a widespread tool in cell identification, particularly in the nervous system, based on the notion that genomic information determines cell identity. However, many cell-type classification studies are unconstrained by other cellular attributes (e.g., morphology, physiology). Here, we systematically test how accurately transcriptional profiling can assign cell identity to well-studied anatomically and functionally identified neurons in 2 small neuronal networks. While these neurons clearly possess distinct patterns of gene expression across cell types, their expression profiles are not sufficient to unambiguously confirm their identity. We suggest that true cell identity can only be determined by combining gene expression data with other cellular attributes such as innervation pattern, morphology, or physiology.

\section{Introduction}

Unambiguous classification of neuronal cell types is a long- standing goal in neuroscience with the aim to understand the functional components of the nervous system that give rise to circuit dynamics and, ultimately, behavior (Stevens, 1998; Masland, 2004; Cuevas-Diaz Duran et al., 2017; Zeng and Sanes, 2017; Luo et al., 2018; Tasic, 2018). Beyond that, agreement upon neuronal cell types provides the opportunity to greatly increase reproducibility across investigations, allows for evolutionary comparisons across species 
(Tessmar-Raible et al., 2007; Tomer et al., 2010), and facilitates functional access to, and tracking of, neuron types through developmental stages (Mehta et al., 2019). To this end, attempts at defining neuronal identity have been carried out using morphology, electrophysiology, gene expression, spatial patterning, and neurotransmitter phenotypes (Whitaker et al., 2011; Cadwell et al., 2015; Zeisel et al., 2015; Chung et al., 2017; Boldog et al., 2018; Ho et al., 2018; Parmhans et al., 2018; Shrestha et al., 2018; Södersten et al., 2018). Since the earliest efforts to capture the transcriptomes of single neurons, using linear or PCR amplification of messenger RNA (mRNA) followed by either cDNA library construction (Eberwine et al., 1992) or microarray hybridization (Tietjen et al., 2003; Esumi et al., 2008; Whitaker et al., 2011), singlecell RNA sequencing (scRNA-seq) (Tang et al., 2009) has become the method of choice for many genome-scale investigations into neuron cell type. Advances in microfluidics, library preparation, and sequencing technologies have propelled an explosion of molecular profiling studies seeking to use unique gene expression patterns to discriminate neuronal types from one another, whether for discovery of new types or further classification of existing ones (Doyle et al., 2008; Haas et al., 2013; Shin et al., 2014; Macosko et al., 2015; Trapnell, 2015; Usoskin et al., 2015; Cembrowski et al., 2016; Crocker et al., 2016; Gokce et al., 2016; Poulin et al., 2016; Tasic et al., 2016; Wagner et al., 2016; Davie et al., 2018; Zhou and Matsunami, 2018). Molecular profiling approaches to tackle the problem of neuronal cell identity have many advantages: First, single-cell transcriptomic data contain 
thousands of measurements in the form of gene products that can be used both in a qualitative (in the form of marker genes) and quantitative (in the form of absolute transcript counts) manner (Luo et al., 2018). Second, scRNA-seq allows for very high-throughput processing of samples with hundreds, if not thousands, of single cell transcripts simultaneously using barcoding techniques (Rosenberg et al., 2018). Third, these techniques can be applied to species that lack wellannotated transcriptomic information, as the cost to generate de novo reference transcriptomes has decreased dramatically in recent years (Reuter et al., 2015). Even the sequencing of heterogeneous tissues from the central nervous system (CNS) can be used in conjunction with predictive modeling to reconstruct markers for major classes of CNS cell types, as has been done with oligodendrocytes, astrocytes, microglia, and neurons, in both humans and mice (Kelley et al., 2018). Classifying neurons into different major categories (such as excitatory vs. inhibitory, parvalbumin+ vs. parvalbumin-, etc.) using qualitative expression measures is an easier task than quantitative approaches that separate neurons into smaller subclasses, but runs into limitations as to how far further classification can proceed. Subclasses of neuron types likely require greater depth of sequencing to resolve, and these neurons are more likely to be defined by the expression of multiple genes rather than unique markers (Hobert et al., 2010). Yet this also is an inherent limitation of scRNA-seq: Low abundance transcripts are often missed or inaccurately classified as differentially expressed (Sha et al., 2015), and methods to dissociate and isolate cells can alter their 
transcriptomic profiles before they are even measured (van den Brink et al., 2017; Harris et al., 2019).

There have now been many studies seeking to determine how many transcriptomically defined cell types might be present in a given part of the brain. For instance, an initial study of the cell-type diversity of the mouse primary visual cortex revealed 42 neuronal and 7 nonneuronal cell types (Tasic et al., 2016). More recent work from the same group identified 133 transcriptomic cell types (Tasic et al., 2018). Work in the retina has led the way as an example of generating a cell-type consensus with an unknown endpoint. Multimodal information of retinal ganglion cell properties, including morphology, physiology, gene expression, and spatial patterning, has converged on over 65 cell types in the macaque fovea and peripheral retina (Peng et al., 2019). However, not all systems have the same technical advantages as the retinal ganglion cells (such as uniform spatial patterning) that can be indicative of cell type, and multimodal information can be more difficult to obtain than high-throughput transcriptomic profiling methods. Therefore, the reliability of transcriptomic profiling with respect to neuronal identity requires additional evaluation.

In this study, we validate and compare transcriptional profiling via scRNAseq and quantitative RT-PCR (qRT-PCR) methods, using supervised and unsupervised analyses, in 2 model systems in which neurons are unambiguously identified based on electrophysiological output, synaptic connectivity, axonal projection, and innervation target: The stomatogastric (STG) and cardiac ganglia (CG) of the crab, Cancer borealis. This approach allows us to test directly how 
much of the known functional and anatomical identity of a neuron is captured in the transcriptomic profile of single neurons within a given network.

\section{Results}

Molecular Profiling of Single Identified STG and CG Neurons by RNASeq.

Because of their large individual cell body size and our ability to manually collect single identified STG neurons (Fig. 4.1), we generated transcriptomes for pyloric dilator (PD; $n=11)$, gastric mill $(G M ; n=11)$, lateral pyloric $(L P ; n=8)$, and ventricular dilator (VD; $\mathrm{n}=8$ ) neurons by typical library preparations rather than more automated procedures such as Drop-seq, Splitseq, or 10× Genomics (Ziegenhain et al., 2017). Sequencing data were mapped to the $C$. borealis nervous system transcriptome (Northcutt et al., 2016b). After removing transcripts for which there was no expression in any cell type, the dataset contained 28,459 distinct contigs (contiguous sequences) in the complete RNAseq dataset. These contigs represent more than the full set of genes transcribed in these cells, as multiple contigs may map to a single gene but during transcriptome assembly the intervening sequence could not be resolved to assemble these distinct fragments (see ref. (Weimann et al., 1991)). We began our analysis of these data using unbiased hierarchical clustering methods, as is commonly done. Using the complete dataset (referred to as "all expressed contigs"), hierarchical clustering (with data centered and scaled across contigs) resulted in 5 clusters (Fig. 4.2A) that appeared not to segregate by cell type. One exception was observed among PD cells. All but 2 PD cells fell within 1 distinct cluster, albeit with a GM cell also identified in this cluster (Fig. 4.2A). While not 
surprising, the complete cellular transcriptome on its own does not distinguish cell types.

We identified and extended our unbiased analysis to the most variably expressed genes in the RNA-seq dataset. The first subset represents the top 2,000 most variable contigs (referred to as the "2000 Highest Variability (H2K) contigs" and the second subset includes variable genes identified using a method described by Brennecke et al. (Brennecke et al., 2013), assuming a false discovery rate (FDR) of 0.2 , which resulted in 922 contigs (referred to as highly variable gene contigs [HVG contigs]). Focusing on variably expressed contigs improved clustering with respect to cell identity, with the HVG dataset outperforming the H2K. In the HVG clustering (Fig. 4.2B), 8/11 GM cells, 5/8 VD cells, 5/8 PD cells, and 5/8 LP cells formed distinct clusters. However, these nodes are not perfectly segregated by cell type and cells of each kind fail to appropriately cluster. If blind to these cell types, the HVG clustering analysis yields 5 to 6 distinct cell-type clusters, rather than the appropriate 4 (Fig. 4.2B). Although differential expression (DE) analysis can only be carried out with a priori knowledge of cell identity or some other post hoc feature by which samples can be grouped, in an attempt to achieve the best performance possible with scRNA-seq clustering analyses we unblinded the analyses to cell type and selected only differentially expressed transcripts. We selected 2 pools of differentially expressed transcripts: Those with a q value $<0.2$ (referred to as "DE0.2") or q value $<0.05$ ("DE0.05"). DE analysis with a q-value cutoff of 0.2 identified 137 transcripts (DE0.2), while a q value of 0.05 identified only 45 
transcripts (DE0.05). Hierarchical clustering of the DE0.2 dataset resulted in better clustering but still failed to faithfully recapitulate cell identity. Hierarchical clustering was greatly improved by using the DE0.05 dataset (Fig. 4.2C) but remained imperfect.

To reveal which preprocessing and clustering methods best recapitulate the predicted number of clusters based on known cell identity, we applied 8 cluster estimation algorithms (optCluster package) (Sekula et al., 2017) on the DE0.05 dataset (centered and scaled by contig, Ward.D2 linkage, and a correlation dissimilarity matrix; Fig. 4.2D). The highest performing clusterings using the DE0.05 data resulted from using Ward.D with a correlation distance metric, resulting in a Jaccard index of 0.738 . The results of cluster estimation differed based on the preprocessing of the datasets. Cluster estimation algorithms were selected from a set of 10 algorithms for use with continuous data as they all yielded usable output. We retained the top 3 predicted $k$ values from each. When data were centered and scaled by contig (Fig. 4.2D), the mode number of clusters estimated was 3 ( 5 indices) and 5 ( 5 indices), and none predicted the correct number of 4 clusters.

Finally, to assess whether unblinded analyses could predict cell type, we tested the ability of 8 supervised machine learning (sML) classification algorithms (generalized linear model [GLM], k-nearest neighbors [kNNs], neural network [NN], multinomial neural network [MNN], random forest [RF], support vector machine with a linear kernel [SVML], support vector machine with a radial kernel [SVMR], and linear discriminant analysis [LDA]) to sort cells based on their 
transformed or untransformed mRNA abundances. Each model's accuracy on new data were estimated using 5 -fold cross-validation. To capture the variation in the All Expressed Contigs dataset, we transformed the data with principal component analysis (PCA) and used the first 38 principal components, which accounted for over $99 \%$ of the variation. The sML mean accuracies on the All Expressed Contigs (PCA transformed) dataset were extremely low, with a maximum mean accuracy of $48.6 \%$ (Fig. 4.2E). sML accuracies improved substantially when classifying the RNA-seq data preprocessed to identify variably expressed contigs (H2K, HVG) and DE contigs (DE0.2, DE0.05), often producing $100 \%$ accuracy for several folds during 5 -fold cross-validation (Fig. 4.2E). It should be noted that no method classified all 5 folds with complete accuracy, even with only DE contigs—-most methods ranged between 75 and $100 \%$ accuracy.

While these results are encouraging, even under optimal conditions (transcriptomic data, selection of transcripts by differential expression, ability to use supervised methods) we were unable to consistently classify these neurons with $100 \%$ accuracy.

Principal Component Analysis of scRNA-Seq Datasets.

PCA is often used to determine whether the variance seen among transcript abundances can be used to separate cells into discrete types. Thus, we performed PCA on the 4 RNA-seq datasets (H2K, HVG, DE0.2, and DE0.05) to examine the ability of this approach to discriminate among cell types (Fig. 4.3). For most of these datasets, the first principal component (PC1) accounted for 
$>40 \%$ of the explained variance, with the exception of the HVG dataset (Fig. 4.3). As such, we have listed the top 10 contigs contributing to variation in PC1 for all 4 datasets in SI Appendix, Table 4.S1. We generated pairwise plots of all 3 PCs in attempts to visualize separation of samples into distinct cell types. There is little ability to resolve cell-type differences in the H2K and HVG datasets (Fig. 4.3 A and B). However, the differentially expressed transcripts allow for some separation of cell type (Fig. 4.3 C and D), with PD becoming somewhat distinct, for example, in the DE0.05 dataset (Fig. 4.3D).

Gene Ontology Analyses of RNA-Seq Datasets.

To determine the types of genes represented in the most variable $(\mathrm{H} 2 \mathrm{~K}$ and HVG) and differentially expressed (DE0.2, DE0.05) datasets among cell populations, we performed gene ontology (GO) enrichment analysis using analysis tools from the PANTHER Classification System (Mi et al., 2012). Because there is relatively little gene annotation work in the crab, we performed GO analysis by first using BLAST to find the top Drosophila ortholog for a given contig, and then retrieving the GO terms associated with this ortholog for analysis. Thus while this analysis provides interesting insight into cell-type specific differences in gene expression, there are limitations to the interpretation, particularly with regards to fold enrichment in Drosophila relative to crab. The most robust expression differences (highest fold enrichment) in the $\mathrm{H} 2 \mathrm{~K}$ molecular function dataset were those of ATP-synthase activity and clathrin binding (SI Appendix, Table 4.S2). Others of note include mRNA 3'-UTR binding, cell adhesion molecule, and calcium ion binding (SI Appendix, Table 4.S2). More 
resolution is gained by examining the biological process category, where $\mathrm{H} 2 \mathrm{~K}$ contigs were most overrepresented for "regulation of short-term neuronal synaptic plasticity," "positive regulation of neuron remodeling," "substrate adhesion-dependent cell spreading," and "clathrin-dependent synaptic vesicle endocytosis" categories (SI Appendix, Table 4.S3) among many others. The HVG dataset shows relatively few enriched categories (SI Appendix, Tables 4.S4 and 4.S5) with FDR correction employed, including ATP binding and transferase activity (related to acetylcholine synthesis).

The differentially expressed contigs of the DE0.2 dataset showed no significantly enriched contigs with FDR employed. Without any $P$ value correction, a number of molecular function categories appear as enriched (SI Appendix, Table 4.S6). However, this is less an appropriate enrichment analysis (due to the relatively small number of contigs) and more a description of gene categories present in the DE0.2 contigs. The top several hits are all indicative of transmitter phenotype, particularly acetylcholine synthesis (SI Appendix, Table 4.S6). However, other receptor activity is represented, such as GABA-gated chloride channel and GABAA receptor activity. Finally, cell-cell adhesion mediator activity appears once again in this list.

Molecular Profiling of Single Identified STG and CG Neurons Using Candidate Genes.

One class of genes that we were surprised to not see represented in DE analyses was the voltage-gated ion channels. A recent study found that 3 classes of neuronal effector genes-ion channels, receptors, and cell adhesion 
molecules - have the greatest ability to distinguish among morphologically distinct mouse cortical cell populations (Sugino et al., 2019). Our previous work also suggests that differential expression of ion channel mRNAs in STG cells may give rise to their distinct firing properties (Schulz et al., 2007c; Temporal et al., 2014b; Santin and Schulz, 2019b). We therefore examined these scRNA-seq data for expression of ion channel mRNAs. Overall, while the sequencing captured most of the known voltage-gated channel subtypes known in C. borealis, raw counts were very low (SI Appendix, Fig. 4.S1). Therefore, we decided to use a qRT-PCR approach to directly test the hypothesis that channels and transmitter receptors are effective genes of interest to differentiate known neuron subtypes.

To examine the molecular profile of individual identified neurons with qRTPCR, we targeted the following transcripts: ion channels, receptors, gap junction innexins, and neurotransmitter related transcripts. These cellular components are responsible for giving neurons much of their unique electrophysiological outputs. As such, we predicted that correspondingly unique expression patterns for this gene set would be present in each neuron type. Using multiplex qRT-PCR, we measured the absolute copy number of 65 genes of interest (SI Appendix, Table 4.S7) from 124 individual STG neurons of 11 different types (10 STG neuron types: pyloric dilator [PD], lateral posterior gastric [LPG], ventricular dilator [VD], gastric mill [GM], lateral pyloric [LP], pyloric [PY], inferior cardiac [IC], lateral gastric [LG], median gastric [MG], dorsal gastric [DG], and the large cell [LC] motor neurons from the cardiac ganglion) ( $n=10$ to 15 per type). We used 
various methods of unsupervised clustering to generate the "best" clustering of these cells based on a priori known number of cell types. This included substituting any missing values in the qRT-PCR dataset via median interpolation.

We then used k-means, unsupervised hierarchical, and shared nearest neighbor-Cliq (SNN-Cliq) clustering to generate unbiased clustering analyses based on expression of these genes of interest. Initial interrogation focused on data transformations with a fixed hierarchical clustering scheme (Ward.D2, correlation dissimilarity matrix as for the scRNA-seq analysis). Unscaled data, as well as data centered and scaled by gene, resulted in different hierarchical clustering patterns. Using unscaled data, hierarchical clustering performed rather poorly in terms of generating distinct clusters that match known cell identity. Performance—as assessed by Jaccard index—was improved by scaling data across genes, generating 8 distinct nodes with high bootstrap support in hierarchical clustering that capture some of the features of known cell identity (LC, IC, LG, LPG, VD, GM, LP, and PD; Fig. 4.4A). However, multiple cell types fall into clusters that either do not show any separation by neuron identity (DG, MG, and PY) or show no bootstrap support based on hierarchical clustering (approximately unbiased $[\mathrm{AU}] \mathrm{P}$ value $=0$ ).

We sought to determine the upper bound for clustering performance with this dataset. If the known anatomical and physiological cell identity is reflected in the ion channel and receptor mRNA profile of STG neurons, then clustering analyses performed on these mRNA data should yield 11 distinct clusters. To determine the feasibility of clustering to sort cell types, we tested 291 clusterings 
(varying clustering methods, distance metrics, and neighbors considered) for each dataset. Each clustering was compared against the known cell identities with the Jaccard index, which ranges from 0 to 1 , where 1 is perfect correspondence between clusterings - in this case, the clustering and cell identity. The best performing combination was data scaled by target and processed using Ward.D2 hierarchical clustering with a correlation distance matrix (Jaccard $=0.636)$. By contrast, the next best clusterings, Ward.D on correlations and Ward.D on data scaled and PCA transformed using Canberra distance, only achieved Jaccard indices of 0.592 and 0.509 , respectively. The 3 least performant methods were single-linkage hierarchical clustering with distance metric of uncentered sample correlation (0.087), maximum distance (0.088), and correlation distance (0.089) metrics. Examining the best performing clustering reveals that LP, PD, LG, IC, DG, LC, PY, GM, LPG, and VD separate fairly well.

Given that an a priori known number of cell types represented in a sample is rare, we tested whether we would have arrived at the correct number of cell types in the sample had we been blind to their identity. We used the best performing transformations from the clustering analysis, i.e., data centered and scaled by gene and a correlation dissimilarity matrix, and 8 cluster determination indices provided by the optCluster package (Sekula et al., 2017). We allowed a minimum of 2 and a maximum of 32 clusters for this and later cluster determination analyses. The mode of the top 3 predicted $k$ values for 8 different methods of cluster estimation was 2 ( 6 indices), followed by 4 (the expected 
number of clusters), and 6 ( 3 indices each) (Fig. 4.4B). If a researcher were using any 1 of these, or a majority vote of several, the chance they would conclude the correct number of 11 clusters are present would be vanishingly low.

We repeated sML analyses on the qRT-PCR data to examine the "best case scenario" performance for clustering analyses. Performance varied substantially between algorithms (e.g., NN achieved a mean accuracy of $43.5 \%$, whereas SVML produced a mean accuracy of $87.5 \%$ ) and was affected by whether the data were centered and scaled (e.g., NN improved by $43.5 \%$, SVML did not improve) (Fig. 4.4C). The highest mean accuracy we achieved was $87.5 \%$ (SVML, either with or without scaling). We considered a principal component transformation as well, but it improved the maximum mean accuracy little (NN, 87.9\%) and worsened the previously most performant methods (SVML decreased from 87.5 to $66.5 \%$, unscaled and $67.4 \%$, scaled). Although neither produces the highest mean accuracy, RF (87.2 to $83.2 \%$ ), GLM (86.6 to $79.2 \%$ ), and LDA (81.9 to $77.7 \%)$ performed consistently across transformations, but clearly not equally well. Overall, the top performing accuracy methods involved centering and scaling the data across genes, and yielded similar efficacies across algorithms (Fig. 4.4C).

Finally, we repeated the PCA to determine if the variance seen among transcript abundances can be used to separate these 11 cell types into discrete clusters. The first 2 principal components (PC1 and PC2) generated from the qRT-PCR data accounted for $31.2 \%$ and $16.6 \%$ of the variance, respectively (Fig. 4.4D). PC3 accounted for $9.6 \%$ of the variance across samples. The top 10 
mRNAs contributing to each of these PCs are listed in SI Appendix, Table 4.S1. We generated pairwise plots of all 3 PCs in attempts to visualize separation of samples into distinct cell types. The most consistent result across all comparisons was that LC neurons from the cardiac ganglion formed a cluster that had less overlap with STG neurons than STG neurons did with each other, particularly in the dimension of PC1 vs. PC2 (Fig. 4.4D). Visualizations of PC1 vs. PC3 and PC2 vs. PC3 also give some indication that even with these target genes of interest, we are able to resolve some separation of these groups (Fig. 4.4D). However, without such extensive a priori knowledge about cell type overall, it is difficult to see how PCA would be effective in separating these 11 cell types based on the expression data at hand.

\section{Comparison of qRT-PCR and RNA-Seq Results.}

To ensure that the RNA-seq and qRT-PCR data were producing comparable expression results, we identified 4 different transcripts that were represented both in the DE dataset from the RNA-seq and the qRT-PCR dataset for the 4 cell types used in RNA-seq (PD, LP, GM, and VD). Overall, there is very strong agreement in expression patterns for all 4 genes (Fig. 4.5A), adding confidence to the quality of both datasets with respect to capturing native expression patterns. We then extracted the RNA-seq expression data for all 65 of the transcripts used in the qRT-PCR dataset. When we performed hierarchical clustering analysis and PCA using these 65 channel and receptor transcripts, the qRT-PCR clustered with nearly $100 \%$ success (with the exception of 2 GM neurons) into nodes that contain the 4 known distinct cell types, while the RNA- 
seq dataset using the same transcripts failed to generate coherent cell type clusters (Fig. 4.5 B and C). As we examined this further, we realized that the 4 transcripts in Fig. 4.5A (ChAT, vAChT, NMDA2B, and KCNK1) represent somewhat higher abundance transcripts that were differentially expressed and showed consistent patterns between qPCR and RNA-seq methods. Other highly expressed transcript types were not differentially expressed (e.g., NaV and INX1-3), and therefore do not contribute strongly to distinguishing cell identity. Conversely, many of the other transcripts in the qRT-PCR dataset that were distinct across cell types had very low levels of detected expression in the RNAseq dataset (SI Appendix, Fig. 4.S1).

\section{Discussion}

Many projects currently attempting to describe neuronal cell types begin with the acquisition of molecular profiles from populations of unidentified neurons (Usoskin et al., 2015; Tasic et al., 2016; Li et al., 2017). Our results demonstrate the strengths and limitations of both unsupervised and supervised methods that rely solely on a molecular profile to recapitulate neuron identity by working "backwards" from an unambiguously known cell identity in a system with a rich history of single-cell neurophysiological characterization, the crustacean stomatogastric ganglion. The analyses clearly demonstrate that even with the most complete a priori knowledge of cell type, there are limitations to determining cell identity through mRNA expression profiles alone. However, these data add to compelling supporting evidence that the molecular profile can partially indicate 
identity, particularly once supervised methods incorporating known cell identification are employed.

Physiological Insights into STG Network Function and Cell Identity.

It can be problematic to infer physiological properties associated with mature protein function from steady-state mRNA levels. Nevertheless, we did make some observations by comparing gene expression profiles to known STG neuron physiology that could have broader implications. First, despite the fact that PD and LPG cells are strongly electrically coupled and fire in a tightly phaselocked fashion when the gastric mill rhythm is not active (Hooper et al., 1986; Weimann et al., 1991; Weimann and Marder, 1994), PD and LPG were about as different from one another based on the outcome of hierarchical clustering of qRTPCR data as they could be (Fig. 4.4A). One might predict that cells with very similar physiological outputs would likely have similar patterns of channel and receptor expression (Schulz et al., 2007c), either because their similar physiology reflects common ontogeny (Farrell et al., 2018), or activity-dependent feedback shapes expression in a conserved fashion (see ref. (Santin and Schulz, 2019b)55); however, neither of these are supported by the data. Futhermore, we would not predict from our results that PD and LPG have a similar developmental trajectory—although their outputs are quite similar—nor are these data consistent with common rules for activity-dependent feedback to the level of steady-state mRNA (O'Leary et al., 2013b, 2014b; Temporal et al., 2014b).

Second, among the full set of STG neurons in the qRT-PCR dataset, we did not see clustering that was faithful to neurotransmitter phenotype. For 
example, 2 of the most closely related neurons in terms of clustering were PD and LP (Fig. 4.4A). Yet these 2 neurons are cholinergic and glutamatergic, respectively. Therefore, it raises a thought-provoking question regarding cell identity. That is, if 2 neurons were similar in most characteristics, yet release distinct transmitters, then should these be considered more distinct classes of cells than those that release a common transmitter but share far fewer other characteristics? Transmitter phenotype is a common distinguishing feature for assigning cell identity (Deneris and Hobert, 2014); yet even this defining feature is not necessarily fixed for the life of the cell (Spitzer, 2017).

Finally, the RNA-seq data and subsequent gene ontology analysis yielded a strong indication that some of the most commonly differentially expressed transcripts represented biological processes associated with synaptic plasticity and neuronal and substrate/cell adhesion remodeling (SI Appendix, Table 4.S3). This is in contrast to the lack of differential expression in this dataset among gene families more directly associated with direct membrane voltage and physiological output, such as channels and receptors. This suggests that a key feature of these networks may reside more in the ability to tune and adapt synaptic connectivity to generate and maintain appropriate network output, rather than to tune individual neuronal excitability (Prinz et al., 2004)—although these are certainly not mutually exclusive (Turrigiano, 2012; Schulz and Lane, 2017).

\section{General Insights.}

There is increasing evidence that discrete classes of genes may distinguish cell types. For example, genes underlying synaptic transmission 
machinery were crucial for separating mouse cortical GABAergic neurons into different types (Paul et al., 2017). Sets of genes that are regulated together that can be thought of as a "gene batteries" have also been shown to be indicative of cell type. For example in Caenorhabditis elegans there is expression of neurontype-specific combinations of transcription factors (Deneris and Hobert, 2014). Recently, 3 classes of neuronal effector genes_ion channels, receptors, and cell adhesion molecules-were determined to have the greatest ability to distinguish among genetically and anatomically defined mouse cortical cell populations (Sugino et al., 2019). Consistent with this work, GO analysis of the 2,000 most variable contigs in the scRNA-seq dataset $(\mathrm{H} 2 \mathrm{~K})$ revealed that the top 5 biological process terms that were significantly enriched included "regulation of short-term neuronal synaptic plasticity," "substrate adhesiondependent cell spreading," and "clathrin-dependent synaptic vesicle endocytosis." Specifically, the differentially expressed contigs dataset (DE0.2) revealed molecular function enrichment for terms related to transmitter identity ("choline:sodium symporter activity" and "acetylcholine transmembrane transporter activity" among others), specifically identified 2 GABA receptor function terms ("GABAgated chloride ion channel activity" and "GABA-A receptor activity") and also included "cell-cell adhesion mediator activity." Finally, our entire qRT-PCR experiment focused on the expression of ion channels, receptors, gap junction innexins, and neurotransmitter-related transcripts. While these 65 genes were not sufficient for classifying cells perfectly into known types, this modest number of transcripts discriminated neuron types fairly well. Thus, 
categorical families of neuronally expressed genes may yield the most useful data for subdividing neurons into distinct classes or subtypes.

Not every system has the same challenges or advantages in assigning neuronal cell identity. Mouse retinal ganglion cells of the same type are regularly and uniformly spaced throughout the retina, while cells belonging to different types do not exhibit spatial patterning relative to one another and are more randomly distributed (Sanes and Masland, 2015). Molecular classification of neurons in $C$. elegans found that anatomically distinct neurons have correspondingly distinct molecular profiles $>90 \%$ of the time (Hobert et al., 2016). However, 146 distinct molecular profiles were identified from the 118 anatomically distinct neuron classes, indicating the potential for molecular subclassification. This classification relied on hierarchical clustering that was carried out solely on identified reporter genes (most prominently transcription factors) and G protein-coupled receptor ([GPCR]-type sensory receptors) known to be differentially expressed across the 302 neurons of $C$. elegans from Wormbase.org (Harris et al., 2014) and not whole transcriptome molecular profiles. It is reassuring that the expression of a wide variety of reporter genes known to be differentially expressed across a population of neurons can recapitulate cell identity. But, this relies on having an established definition of neuron type to constrain hierarchical clustering, as differential expression analysis can only be carried out by assigning samples to different populations. Our results are consistent with these findings, in that clustering is most reliable when differentially expressed targets are used as the transcriptomic dataset. 
Further, these data also demonstrate that without separating cell types a priori by such additional criteria, molecular cell classification can generate unreliable results, particularly with neurons that belong to the same network.

What are the sources of variability that could mask molecular identification of neuronal identity? Most common high-throughput molecular profiling techniques require destructive sampling to acquire mRNA abundances, which generates only a snapshot of the profile at a single point in time. Gene expression has stochastic characteristics (Raj and van Oudenaarden, 2008; Li and Xie, 2011); transcription takes place not continually, but in bursts of expression (Wang et al., 2018) (reviewed in (Symmons and Raj, 2016)); and steady state mRNA abundances are the result of rates of expression, degradation, and mRNA stability (Perez-Ortin, 2008). Single-cell transcriptomes can be altered biologically as a consequence of activity (Benito and Barco, 2015), injury (Llorens-Bobadilla et al., 2015), long-term memory formation (Crocker et al., 2016), differentiation (Olivera-Martinez et al., 2014), and aging (Moroz and Kohn, 2013; Davie et al., 2018), as well as being affected by technical noise (Brennecke et al., 2013). Cells also belong to different transcriptional states under certain conditions, with the major distinction between a cell type and cell state being that state is a reversible condition, whereas type is more constant and includes neuronal states (Tasic et al., 2017). Neuron types exist in a continuum, exhibiting variation in expression patterns within defined cell types, increasing difficulty in discreetly drawing the cutoff of one type from another (Cembrowski and Menon, 2018). Thus, the assertion that a given neuron 
has a single transcriptomic profile is an oversimplification and simply represents a moment in time in the life of a given cell.

The present study has limitations. The expression of the focal gene set of ion channels, receptors, gap junction innexins, and neurotransmitter-related transcripts examined here ultimately discriminated neuron types fairly well, using supervised methods taking into account known neuron identity. This same gene set did not perform well in the same cell types using RNA-seq (Fig. 4.5), where a lack of low-abundance transcripts (such as transcription factors and ion channels) may have prevented us from robustly identifying cell-type-specific expression patterns; thus, depth of sequencing is always an ambiguity in every RNA-seq study (Rizzetto et al., 2017). Furthermore, while we sampled the mRNA transcriptome of individual neurons, we have not measured other gene products that could drive unique identity, including noncoding RNA species such as microRNA (miRNA) and long noncoding RNA (IncRNA) (Sheng et al., 2017). Epigenetic modifications have also been implicated in neuronal cell identity (Mito et al., 2018), which were not considered in this study. Further, there are numerous other methods and statistical analyses being applied to molecular profiles to distinguish cell type. We focused on the more commonly employed analyses (PCA, hierarchical clustering, and machine learning algorithms) in the literature. Finally, although we are confident in our ability to identify and harvest the targeted neuron types, we cannot entirely rule out the possibility of an occasional misidentified or wrongly isolated cell, as well as the potential presence of adherent support cells. 
This present study reveals the circular nature of using transcriptomics to identify cell types: Molecular profiling is most effective when cells are separated into distinct types a priori, yet this is often not possible in many systems. So then how can we most effectively use molecular profiling on unknown populations of cells? The clear answer is to provide as much multimodal data as possible in the analysis. Here, the additional data were an a priori separation into cell type based on electrophysiological output, synaptic connectivity, axonal projection, and muscle innervation target (Marder and Bucher, 2007). While it has been more difficult to achieve multimodal data integration in systems such as cortex, the approach is gaining traction and proving effective (Kim et al., 2019).

For example, supervised clustering methods proved superior to unsupervised algorithms in separating pyramidal neurons from interneurons in the mouse neocortex based on morphological phenotypes (Guerra et al., 2011). Genetically and anatomically defined cell populations in the mouse cortex have revealed much finer resolution and confidence in molecular profiling (Sugino et al., 2019), and combined physiological and transcriptomic approaches have yielded valuable insights into spinal interneuron diversity as well (Bikoff et al., 2016). Much like a circuit's connectome alone is insufficient to predict network output and function (Gutierrez et al., 2013), so too the transcriptome alone is insufficient to generate a definitive cell type. Yet it also is clear that transcriptome profiling provides valuable insight into understanding the functional role of individual neurons and neuron types in a network. Therefore, increasing evidence indicates that transcriptomic approaches will benefit from integration 
with other modalities of cell-defining characteristics to gain more accurate distinctions among cell types. scRNA-seq data on their own should be viewed with caution with respect to a definitive cell identity assessment until more studies with multimodal integration become available.

\section{Conclusion}

Classification and characterization of cell types often has been performed ad hoc within the context of specific studies or species rather than based on a systematic approach. Without a more systematic attempt to define cell type, it will be challenging to use the extensive data being generated in a comparative fashion to its fullest potential (Tosches and Laurent, 2019). Acknowledging that cell types and their diversity are the product of evolution, Arendt et al. (Arendt et al., 2016) defined a cell type as "a set of cells in an organism that change in evolution together, partially independent of other cells, and are evolutionarily more closely related to each other than to other cells." As a consequence, cells of a given type use certain genomic information — both coding and noncoding that determines cell identity and is not used by other cells. This suggests that single-cell gene expression profiling is a valuable approach to attain a comprehensive understanding of an organism's cellular physiology. As such, cell classification schemes are susceptible to similar limitations as phylogenetic studies. For example, the species concept continues to be an area of active discussion among evolutionary biologists (Freudenstein et al., 2017), and prokaryotic species assignment shares many of the same challenges as single- 
cell eukaryotic cell-identity approaches (Konstantinidis et al., 2006). Yet there are lessons to be carried across these diverse disciplines. Just as the application of molecular characters in phylogenetic analyses was initially met with skepticism, ultimately this approach became an essential scientific discipline, in part due to the value of combined molecular, morphological, and behavioral data (Avise, 1994). Transcriptomic approaches to cell identity already are broadly embraced. However, to fully leverage these kinds of data, it seems prudent to generate a more systematic definition and approach to classifying neuron identity. This definition should strive to combine multiple modalities of data, both to increase confidence in the transcriptomic identification as well as refine and better standardize the definition of what constitutes distinct cell types or unique cell identity.

\section{Methods}

Cell Collection and RNA Preparation.

All animal experiments were approved by the Animal Care and Use Committees at University of Missouri-Columbia and Brandeis University. Adult male Jonah crabs, C. borealis, were purchased from the Fresh Lobster Company (Gloucester, MA) and Commercial Lobster (Boston, MA). Animals were allowed to acclimate to their tanks and kept in filtered artificial seawater tanks chilled at $10^{\circ} \mathrm{C}$ to $13^{\circ} \mathrm{C}$ on a $12 / 12$ light:dark cycle until use. Prior to dissection, crabs were put on ice for 30 min to induce anesthetization.

The complete stomatogastric nervous system (STNS) was dissected and pinned out in a dish coated in Sylgard (Dow Corning) with chilled $\left(12{ }^{\circ} \mathrm{C}\right)$ 
physiological saline (composition in mM/l: $440.0 \mathrm{NaCl}, 20.0 \mathrm{MgCl} 2,13.0 \mathrm{CaCl}$, 11.0 KCl, 11.2 Trizma base, and 5.1 maleic acid $\mathrm{pH}=7.4$ at $23^{\circ} \mathrm{C}$ in RNase-free water). Recordings were made of the spontaneously active stomatogastric rhythms, and all were confirmed to be generating healthy and robust output equivalent to the standard in the extensive literature on this preparation (Marder and Bucher, 2007). This ensured all preparations used in this study were within the realm of normal physiological function. Following desheathing of the STG, neurons were identified by simultaneous intra- and extracellular recordings (Hooper et al., 1986; Weimann et al., 1991). Ten neuron types identified in the STG of C. borealis were targeted for this study: PD, LPG, LP, IC, LG, MG, GM, $P Y, V D$, and DG. Identified neurons were extracted as previously described (Schulz et al., 2006b). More information is provided in SI Appendix, Supplemental Methods. Identified neurons (Fig. 4.1) were immediately placed in a cryogenic microcentrifuge tube containing $400 \mu \mathrm{L}$ lysis buffer (Zymo Research) and stored at $-80^{\circ} \mathrm{C}$ until RNA extraction. Total RNA was extracted using the Quick-RNA MicroPrep kit (Zymo Research) per the manufacturer's protocol.

\section{Library Preparation and Single-Cell RNA-Seq.}

Library construction and RNAsequencing services were carried out by the University of Texas at Austin Genomic Sequencing and Analysis Facility (Austin, TX). Extracted single-cell RNA from identified neurons from the STG was used to generate cDNA libraries using TruSeq Stranded mRNA Library Prep Kit (Illumina, San Diego, CA). Libraries were sequenced in a paired-end 150-bp $(2 \times 150 \mathrm{bp})$ configuration on the NextSeq 500 Illumina platform (Illumina). Raw reads were 
processed and analyzed on the Stampede Cluster at the Texas Advanced Computing Center. Read quality was checked using the program FASTQC. Lowquality reads and adapter sequences were removed using the program Cutadapt (Martin, 2011). The 40 identified neurons used in this study all had at least 4 million uniquely mapped reads per sample, comprising $11 \mathrm{PD}, 11 \mathrm{GM}, 8 \mathrm{LP}$, and 8 VD cell types. These sequencing reads are deposited in the National Center for Biotechnology Information (NCBI) BioProject archive (PRJNA524309) with the following identifiers: BioSample: SAMN11022125; sample name: STG neurons; SRA: SRS4411333.

\section{Mapping and Differential Expression.}

The software package Kallisto (Bray et al., 2016) (v0.43.1) was used in the quantification of RNA-seq abundances through the generation of pseudoalignments of paired-end fastq files to the $C$. borealis annotated nervous system transcriptome (Northcutt et al., 2016b). While a fully annotated genome represents the best reference for mapping, there is no genome yet available for C. borealis. In general, decapod crustacean genomes are severely lacking. The only published decapod genome likely to be of high enough quality for such mapping is that of the marbled crayfish, Procambaurs fallax $\mathrm{f}$. virginalis (Gutekunst et al., 2018). However, as this species likely last shared a common ancestor on the order of 350 mya, we did not feel mapping was likely to be successful. Therefore, we elected to use what is a fairly high-quality transcriptome from C. borealis. Bootstrapping of the quantification was performed iteratively for 100 rounds. Resulting counts were normalized through the 
transcripts per kilobase million (TPM) method. Differential expression analysis was carried out using the software package Sleuth (Pimentel et al., 2017) (v0.30.0) using TPM normalized counts for each cell type.

Gene Ontology Enrichment Analysis.

Because $C$. borealis lacks a well-curated reference genome, GO terms were assigned to the $C$. borealis transcriptome based on best BLASTX hits through reciprocal queries between crab sequence and the Drosophila melanogaster NCBI RefSeq database (release 93). BLAST annotation was carried out based on Drosophila protein sequence using the BLAST2GO (version 5.1) software suite with the blast $x$-fast alignment with an $E$ value threshold $=$ 1.0E-3 to generate $D$. melanogaster NCBI gene IDs associated with each $C$. borealis contig. This produced 1,348 and 252 annotated gene IDs for the H2K and HVG datasets, respectively. These IDs were used as input for statistical overrepresentation tests using the PANTHER Gene Ontology Classification System (v14.1) with default settings using D. melanogaster as the reference species. Molecular function and biological process GO terms were examined for enrichment in these datasets, and results reported reflect FDR correction except where noted.

\section{Multiplex Primer and Probe Design.}

Multiplex primer and probe sequences targeting C. borealis genes were generated using the RealTimeDesign qPCR assay design software from LGC Biosearch Technologies (Petaluma, CA) for custom assays. Multiplex cassettes were designed as a unit to ensure minimal interference in simultaneous $\mathrm{QPCR}$ 
reactions. Probe fluorophore/quencher pairs used in this study are as follows: FAM-BHQ1, CAL Fluor Gold 540-BHQ1, CAL Fluor Red 610-BHQ2, Quasar 670BHQ2, and Quasar 705-BHQ2. Forward and reverse primer pair, as well as associated probe, sequences can be found in SI Appendix, Table 4.S7.

\section{cDNA Synthesis and Preamplification.}

Following RNA extraction, individual neuron RNA samples were reverse transcribed into cDNA using qScript cDNA SuperMix (QuantaBio, Beverly, MA) primed with random hexamers and oligodT per the manufacturer's protocol in 20$\mu \mathrm{L}$ reactions. Half of each resulting cDNA pool $(10 \mu \mathrm{L})$ was preamplified using PerfeCTa PreAmp Supermix (QuantaBio) with a 14-cycle RT-PCR primed with a pool of target-specific primers (SI Appendix, Table 4.S7) in a 20- $\mu$ L reaction per the manufacturer's protocol to allow for enough product to carry out 15 multiplex QPCR reactions per individual neuron sample. Amplified and unamplified target abundances were compared to ensure minimal amplification bias in the preamplification of samples (SI Appendix, Fig. 4.S2).

\section{Quantitative Single-Cell RT-PCR.}

Following preamplification of cDNA, samples were diluted $7.5 \times$ in nuclease-free water ( $150 \mu \mathrm{L}$ final volume) to allow for the quantification of 73 unique gene products across 15 multiplex assays, each able to measure 4 to 5 different transcripts (SI Appendix, Table 4.S7). Reactions were carried out in triplicate on 96 -well plates with $10-\mu \mathrm{L}$ reactions per well using a CFX96 Touch Real-Time PCR Detection System from Bio-Rad (Hercules, CA). Cycling conditions for $\mathrm{QPCR}$ reactions were as follows: $95^{\circ} \mathrm{C}$ for $3 \mathrm{~min}$; 40 cycles of 95 
${ }^{\circ} \mathrm{C}$ for $15 \mathrm{~s}$ and $58{ }^{\circ} \mathrm{C}$ for $1 \mathrm{~min}$. Fluorescent measurements were taken at the end of each cycle. The final concentration of primers in each multiplex qPCR reaction was $2.5 \mu \mathrm{M}$ and $0.3125 \mu \mathrm{M}$ for each probe.

To quantify absolute mRNA abundances, standard curves were developed for each qRT-PCR multiplex assay using custom gBlock gene fragments (Integrated DNA Technologies, Coralville, IA). Standard curves were generated using a serial dilution of gBlock gene fragments from $1 \times 106$ to $1 \times 101$ copies for each reaction assay and shown to be linear and reproducible. Copy numbers were calculated using the efficiency and slope generated from the standard curves and accounting for the 14-cycle preamplification and subsequent cDNA dilution described above.

Statistical Analysis.

Expanded details on these analyses are provided in the SI Appendix, Supplemental Methods.

All statistical analyses were performed using $R$ version 3.5 .3 (2019-03-11) "Great Truth" (R Core Team and Team, 2008). We used single-cell RNA-seq data to evaluate our methods under expected and near best case scenarios. To this end, we reduced the dimensionality of the data (28,695 contigs) by selecting the 2,000 most variable contigs and by selecting 922 highly variable contigs. We selected those contigs differentially expressed at an alpha of 0.2 or 0.05 , centered and scaled these datasets, and used PCA to determine if any of the cell types were visually separable across these subsets of the data. 
Next, we performed cluster estimation using the optClust() function of the optCluster package (Sekula et al., 2017). To assess the performance of unsupervised machine learning methods, we tested several clustering algorithms and clustering methods and selected the high-performing clustering methods based on the Jaccard index calculated against cell identity. We selected one of the best performing combinations (Ward's method with correlation as the distance metric) for visualization.

Finally, we applied several supervised machine-learning methods to evaluate predictive power of expression data in ideal circumstances (i.e., prior knowledge of a given cell type's molecular identity). For each of the models, we tested a variety of tuning parameters and selected the most effective parameter set before comparison with other methods. Methods were evaluated by using cross-validation (with 5 folds) to produce the expected accuracy on new data. The same approaches were applied to the single-cell qRT-PCR dataset, with a few caveats. Given its relatively smaller size, dimensionality reduction was not necessary to overcome technical or practical hurdles. Thus, we tested both the raw and centered and scaled dataset in addition to PCA transformations of the same. We also increased the maximum $\mathrm{k}$ allowed in cluster estimation to 32 


\section{Declarations}

License

This open access article is distributed under Creative Commons AttributionNonCommercialNoDerivatives License 4.0 (CC BY-NC-ND).

\section{Availability of Data and Materials.}

All sequence data can be accessed in National Center for Biotechnology Information BioProject archive (PRJNA524309) with the following identifiers: BioSample:

SAMN11022125; sample name: STG neurons; SRA: SRS4411333. Accession numbers for crab channel and receptor sequences targeted in qRT-PCR experiments are provided in SI Appendix, Table 4.S7.

\section{Acknowledgments}

We thank members of the D.J.S., H.A.H., and E.M. laboratories for helpful discussions. We thank the Genomic Sequencing and Analysis Facility (The University of Texas [UT] at Austin) for library preparation and sequencing and the bioinformatics consulting team at the UT Austin Center for Computational Biology and Bioinformatics for helpful advice. This work was supported by National Institutes of Health grant R01MH046742-29 (to E.M. and D.J.S.) and the National Institute of General Medical Sciences T32GM008396 (support for A.J.N.) and National Institute of Mental Health grant 5R25MH059472-18 and the Grass Foundation (support for Neural Systems and Behavior Course at the Marine Biological Laboratory). 


\section{Author contributions:}

A.J.N., D.R.K., E.M., and D.J.S. designed research;

A.J.N., A.G.O., R.M.H., and J.M.S. performed research;

A.J.N., D.R.K., B.M.G., H.A.H., and D.J.S. analyzed data;

A.J.N., D.R.K., H.A.H., E.M., and D.J.S. wrote the paper.

Reviewers: G.E.R., University of Illinois at Urbana-Champaign; and C.W., Institut du Cerveau et de la Moelle Epiniere.

\section{Competing Interests}

The authors declare no competing interest. 


\section{Supporting Information Appendix}

\section{Supplemental Methods}

Cell collection.

A Vaseline well was constructed around the ganglion, in which $\sim 2.5 \mathrm{mg} / \mathrm{ml}$ protease (Sigma - P6911, St. Louis, MO) in chilled physiological saline was added to disrupt connective tissue and loosen adherent support cells during a 10-15 minute incubation. The well was then thoroughly washed with fresh physiological saline to halt further enzymatic activity and remove any loose support or connective cells. Chilled ethylene glycol was gradually added in increasing concentration until a $70 \%$ solution of chilled ethylene glycol in saline was present to the well. During the process of protease digestion, normal activity continues in the STG (Schulz et al., 2006b), and this activity maintains patterns of gene expression in STG neurons for at least some classes of gene products (Temporal et al., 2014b; Santin and Schulz, 2019b). Furthermore, the time from the initiation of protease digestion to suspension in cold ethylene glycol (which represents a stable end point) is $<45$ minutes. Normal STG output can be maintained for many days (Luther et al., 2003; Hamood et al., 2015), and steady state mRNA levels remain relatively stable for at least 72 hours as long as activity is maintained (Temporal et al., 2014b). When activity is disrupted, significant changes in channel mRNAs can be detected only on the order of 8 hours (Santin and Schulz, 2019b). and our preliminary data show no changes within 2 hours in cardiac motor neurons (LCs; unpublished observation). While we cannot determine whether the collection process does not alter steady state 
mRNA levels for all gene products, present evidence suggests that the time frame over which the neurons are harvested is not likely to substantially change the state of these neurons relative to those in intact ganglia.

Once the ganglia were suspended in cold $70 \%$ ethylene glycol solution, the saline outside the well was replaced with distilled water, and the entire dish was frozen at $-20^{\circ} \mathrm{C}$ for 30 minutes. This constitutes a freeze substitution that allows the easy hand-dissection and removal of single, intact neurons for biochemical and molecular analyses (Marder, 1976). Due to the large size of C. borealis STG neuronal somata (50-150 $\mu \mathrm{M}$ in diameter) (Ransdell et al., 2010), fine forceps were used to manually remove each neuron.

\section{Statistical Analysis.}

All statistical analyses were performed using $R$ version 3.5 .3 (2019-03-11) -- "Great Truth" (R Core Team and Team, 2008). We used single cell RNA-seq data to evaluate our methods under expected and near best case scenarios. To this end, we reduced the dimensionality of the data ( 28,695 contigs) by selecting the 2000 most variable contig and by selecting 922 highly variable contigs selected using the M3Drop implementation of the Brennecke method (Brennecke et al., 2013) (i.e. M3Drop::BrenneckeGetVariableGenes() function (Andrews, 2019)) assuming a 0.2 false discovery rate. To test performance under ideal conditions we selected those contigs differentially expressed at an alpha of 0.2 or 0.05. We centered and scaled the aforementioned datasets and their progenitors via the caret::predict() and caret::preprocess() functions (from Jed Wing et al., 2018). We also tested dimensionality reduction via PCA. We further used PCA in 
exploratory data analysis to determine if any of the cell types were visually separable across four subsets of the data (Seq H2K, Seq HVG, Seq DE0.2, and Seq DE0.05).

Next, we performed cluster estimation using the optClust() function of the optCluster package (Sekula et al., 2017). The algorithms used on each dataset varied by whether the data were counts or continuous. Allowed $\mathrm{k}$ values ranged from 2-10 (i.e. cells in dataset / 4, rounding up). We selected the top three predicted $k$ values from each algorithm for visualization of the spread of predicted ks.

To assess the performance of unsupervised machine learning methods we tested several clustering algorithms - kmeans clustering, hierarchical clustering (using a variety of distance metrics, (euclidean, maximum, manhattan, canberra, binary, minkowski, correlation, uncentered) and clustering methods (ward.D, ward.D2, single, complete, average, mcquitty, median, centroid, ward.D2)), and SNN-Cliq clustering (Xu and Su, 2015). We then selected high performing clustering methods based on the Jaccard index calculated against cell identity. We selected one of the best performing combinations (Ward's method with correlation as the distance metric) for visualization.

We applied several supervised machine learning methods to evaluate predictive power of expression data in ideal circumstances (i.e. prior knowledge of a given cell type's molecular identity). Specifically, we tested elastic regression, k-nearest neighbors, linear discriminant analysis, neural network, multinomial neural network, random forest, support vector machine with a radial 
kernel, and support vector machine with a linear kernel. For each of these models we tested a variety of tuning parameters and selected the most effective parameter set before comparison with other methods. Methods were evaluated by using cross validation (with five folds) to produce the expected accuracy on new data. The same approaches were applied to the single cell RT-qPCR data set, with a few caveats. Given its relatively smaller size, dimensionality reduction was not necessary to overcome technical or practical hurdles. Thus, we tested both the raw and centered and scaled dataset in addition to PCA transformations of the same. We also increased the maximum $\mathrm{k}$ allowed in cluster estimation to 32. 
Figure Legends, Tables, Extended Data, and Multimedia

Figure 4.1 Connectome and representative waveforms of neurons from the stomatogastric and cardiac ganglia
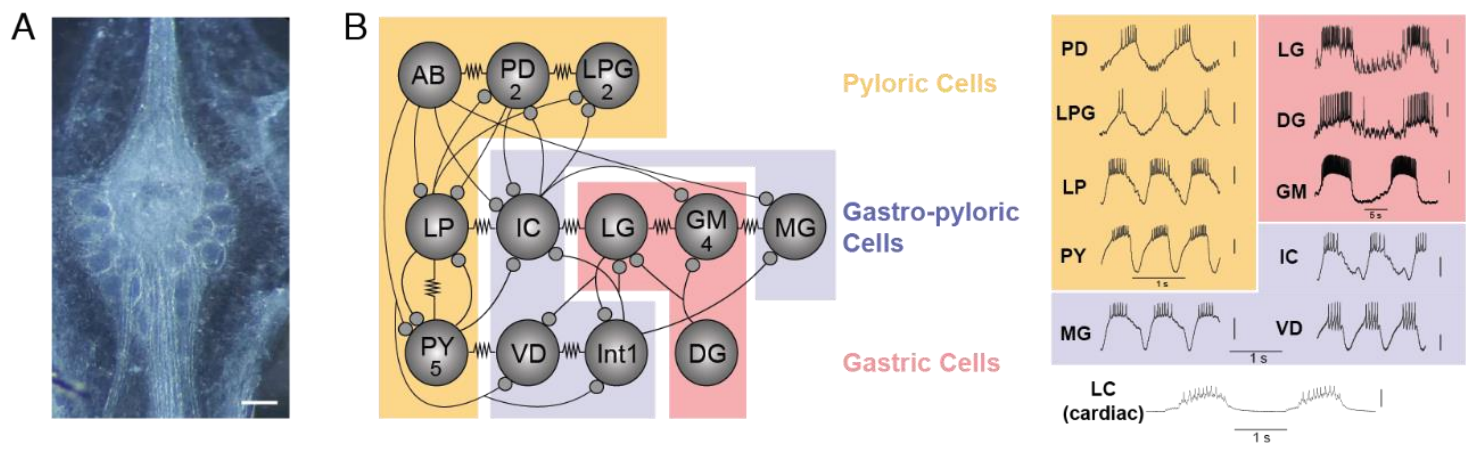

(A) Photomicrograph of the stomatogastric ganglion. (Scale bar, $200 \mu \mathrm{m}$.) (B) Circuit map of the STG. The STG contains 12 cell types that innervate the pylorus and gastric mill of the crab stomach. These cells are individually identifiable, and their chemical (closed circles) and electrical (resistor symbols) synaptic connections are all known. We used 10 of these 12 STG cell types (not AB or Int1) for this study, as well as motor neurons of the cardiac ganglion as an outgroup for comparison. Example traces were taken from intracellular recordings of each of the 11 identified neuron types used in this study. Neurons are involved in 3 different networks/circuits in the crab, C. borealis: the pyloric network (anterior burster (AB), PD, LPG, LP, and PY; orange box), the gastric network (LG, DG, and GM; red box) and the cardiac ganglion network (Bottom). Note the time scale difference in the long-lasting bursts of the gastric cells (red box) relative to the pyloric cells (orange box). Some neurons (interneuron 1 (INT1), IC, VD, and MG) participate in both gastric and pyloric network activity and are noted in the purple box. LC motor neurons of the cardiac ganglion are used as an "outgroup" to compare expression patterns of motor neurons from a distinct ganglion (cardiac ganglion). Each of the representative recordings is independent as an example of individual cell output, 
and simultaneous network activity is not plotted here. Thus, none of the phase relationships of these units within their respective rhythms is implied in any of the recordings. 
Figure 4.2 Efficacy of clustering, cluster estimation, and classification procedures with single-cell RNA-seq measurements.
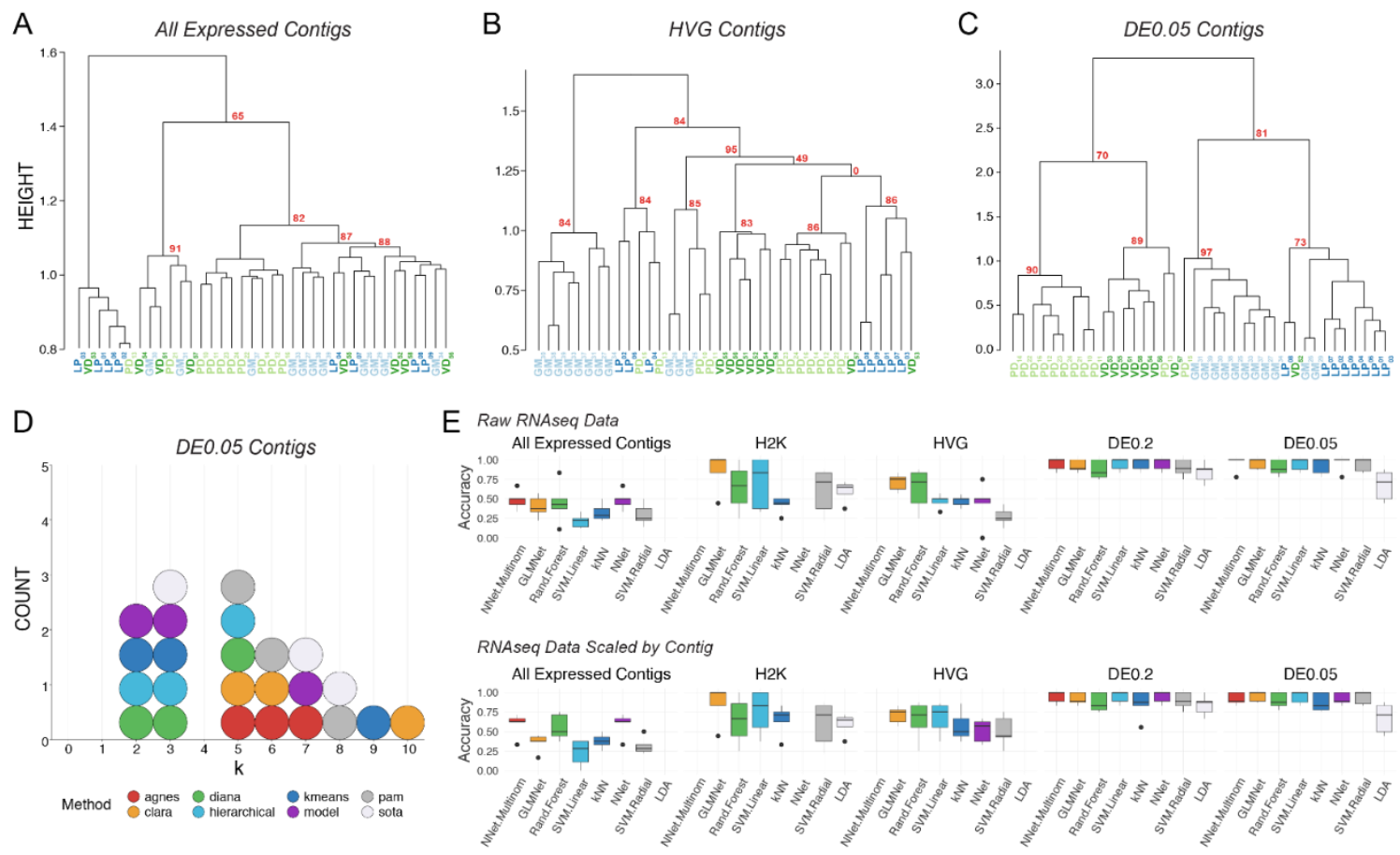

Post hoc recapitulation of cell identity via single-cell RNA-seq with hierarchical clustering and SML algorithms. (A) Hierarchical clustering of cell type with correlation as the distance metric, Ward.D2, as the clustering method, and data centered and scaled by contig for all expressed contigs, $(B) \mathrm{HVG}$ dataset, and $(C)$ DE contigs at the $\mathrm{q}<0.05$ level. Each cell type is color coded, and AU $P$ values are noted for each of the major nodes. Cells are identified by type (LP, PD, GM, VD) and a subscript that denotes a unique sample identifier. $(D)$ Dotplot of the top 3 predicted number of clusters ( $\mathrm{k}$ values) for 8 algorithms. None of these algorithms correctly predicted the expected 4 distinct clusters that would represent the 4 different cell types in this assay. (E) Accuracy (proportion of correctly identified cells) of cell-type prediction using 8 different methods of sML (GLM, kNN, NN, MNN, RF, SVML, SVMR, and LDA) for each of the datasets. Box and whisker plots show the efficacy of these methods to recapitulate cell identity from 
these 2 sets of contigs as estimated by cross-validation ( 5 folds). To assess the efficacy of these methods on the full RNA-seq dataset, we used PCA for dimensionality reduction (i.e., $>28,000$ contigs to 38 PCs) while retaining $99 \%$ of the variance. Results are shown for raw data (Top row) and data scaled across contigs (Bottom row). 
Figure 4.3 Principal component analysis using variable or differentially expressed contigs
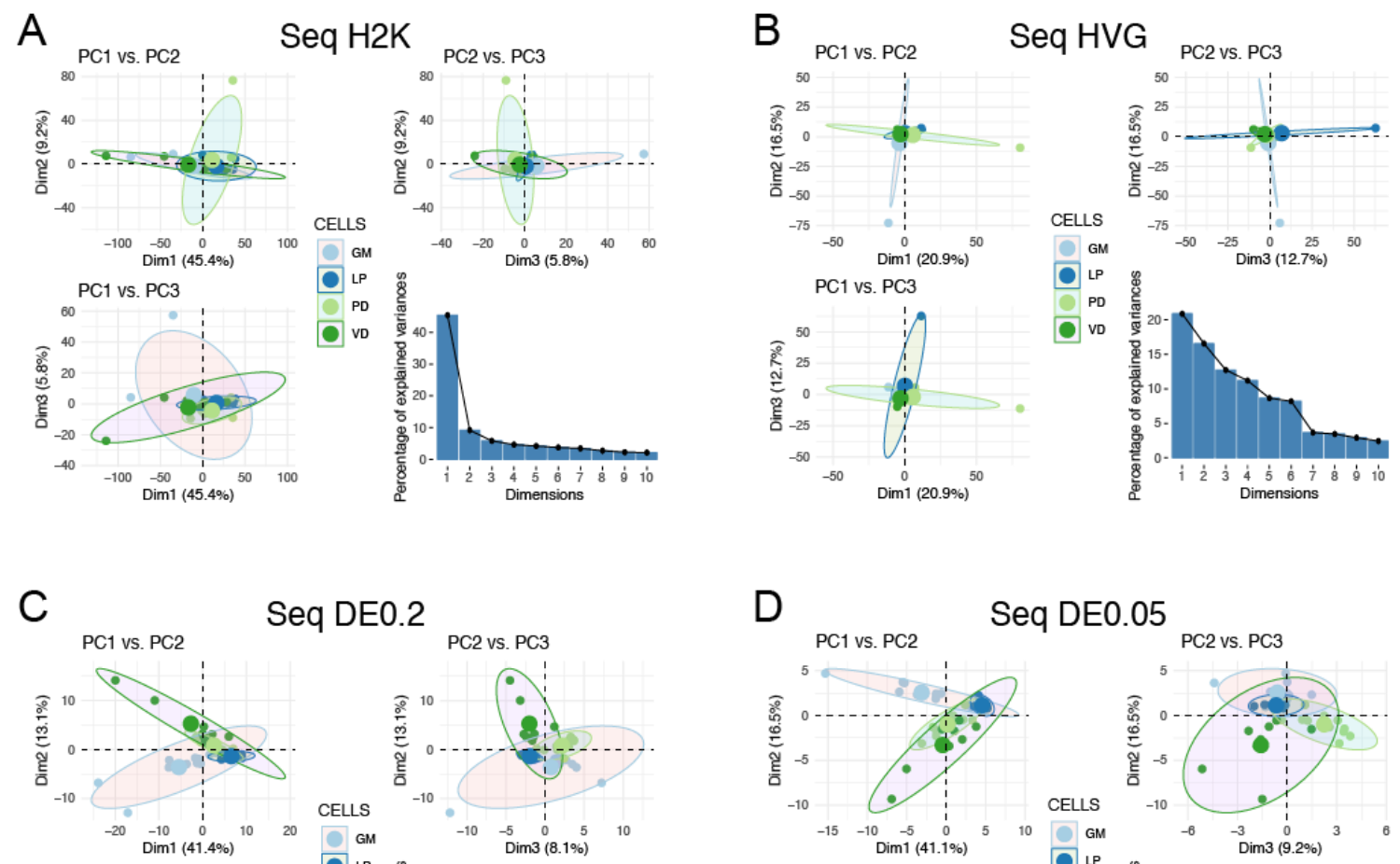

Seq DE0.2
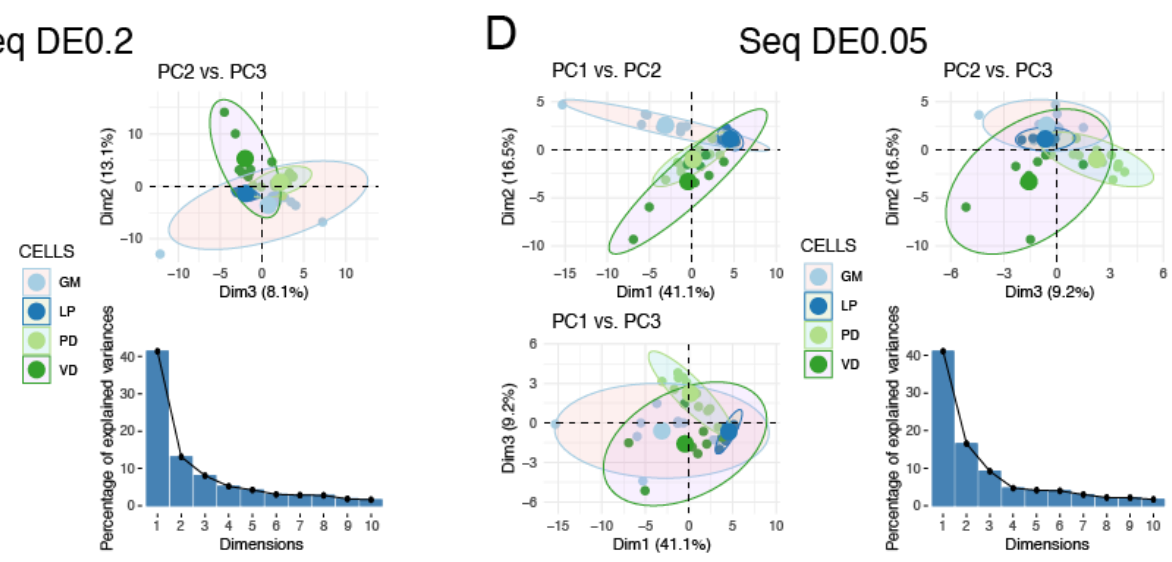

PCA for 4 different RNA-seq datasets. We performed PCA using $(A)$ the 2,000 contigs with the highest variance in expression (H2K), $(B)$ the HVG and DE contigs at the $(C) \mathrm{q}<$ 0.2 (DE0.2), and $(D) \mathrm{q}<0.05$ (DE0.05) levels. For each panel we have plotted pairwise comparisons of PC1, PC2, and PC3, as well as a scree plot representing the percentage of variance explained by PCs 1 through 10 . 
Figure 4.4 Efficacy of clustering, cluster estimation, and classification procedures with single-cell qRT-PCR measurements.

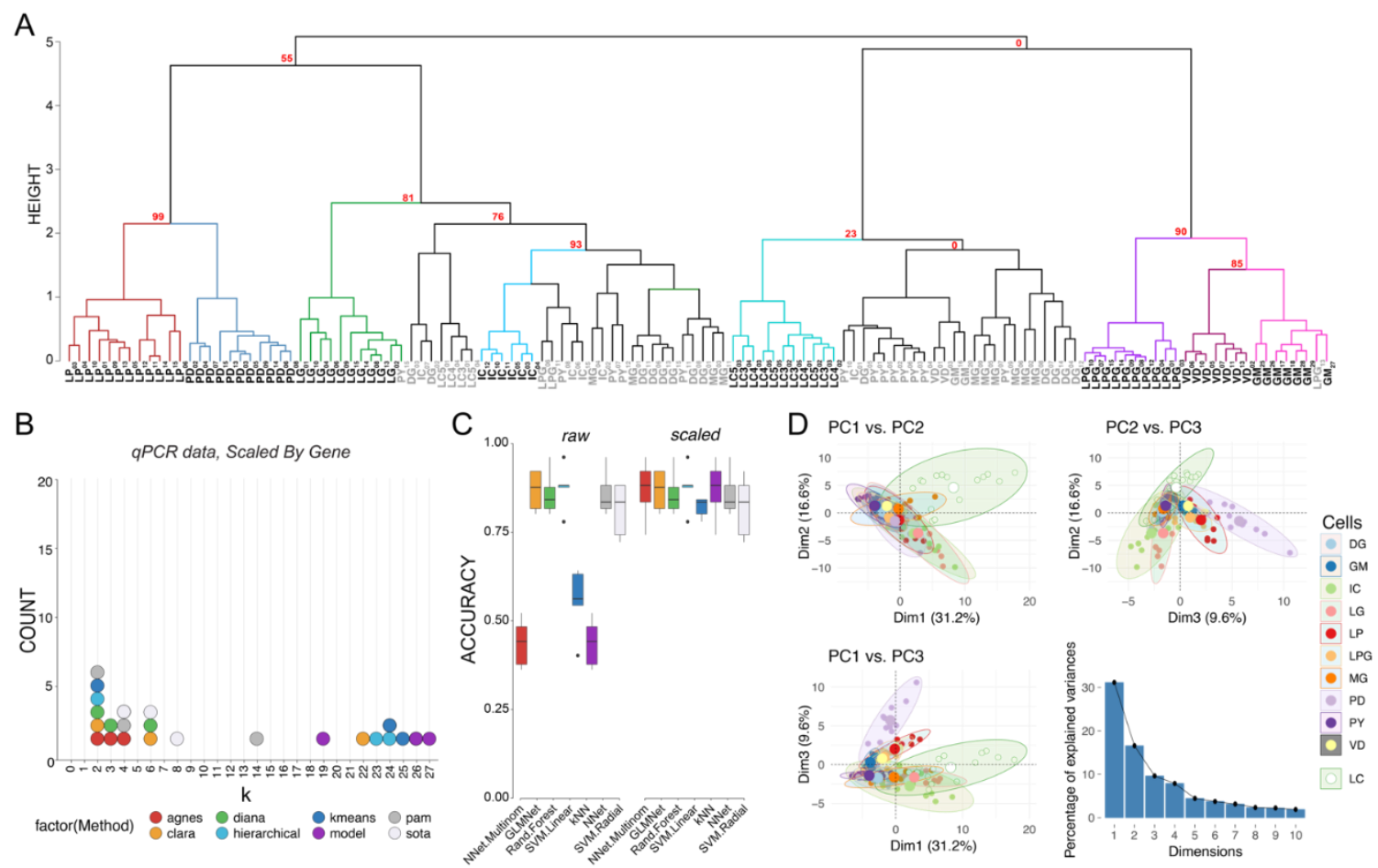

Post hoc recapitulation of cell identity via qRT-PCR expression with hierarchical clustering and sML algorithms. $(A)$ Hierarchical clustering of cell type with correlation as the distance metric and Ward.D2 as the clustering method for data centered and scaled across genes. AU $P$ values for a given node are noted in red. Each node that has $>80 \%$ support by AU $P$ value is color coded, and cell types that form a largely coherent group are noted in bold. Cells that do not appear to cluster by type are noted in gray. Cells are identified by type and a subscript that denotes a unique sample identifier. (B) Dotplot of the top 3 predicted number of clusters based on 8 different prediction algorithms. None of these methods correctly predicted 11 distinct clusters that would represent the 11 different cell types in this assay. (C) Accuracy of cell-type prediction using 8 different methods of SML for each of the datasets. Box and whisker plots show efficacy of each method across 5 cross-validation folds. (D) PCA for qRT-PCR data. Pairwise 
comparisons of PC1, PC2, and PC3 are shown in each panel as in Fig. 4.3. PC1

accounted for $31.2 \%$ of the variance, PC2 accounted for $16.6 \%$, and PC3 accounted for $9.6 \%$ of the total variance across samples. A scree plot shows the amount of variance explained by PCs 1 through 10 . 
Figure 4.5 Expression level and clustering results using qRT-PCR or RNAseq

A
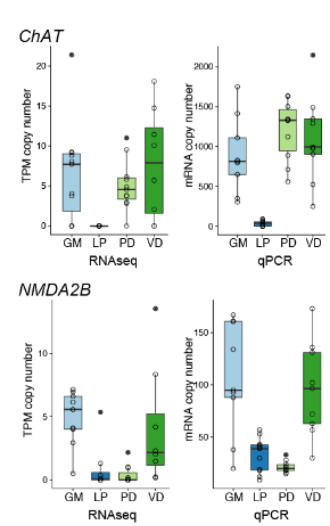

B
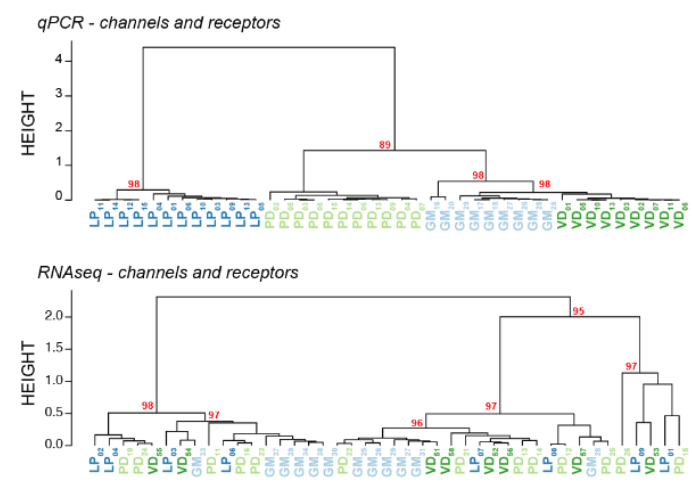

C
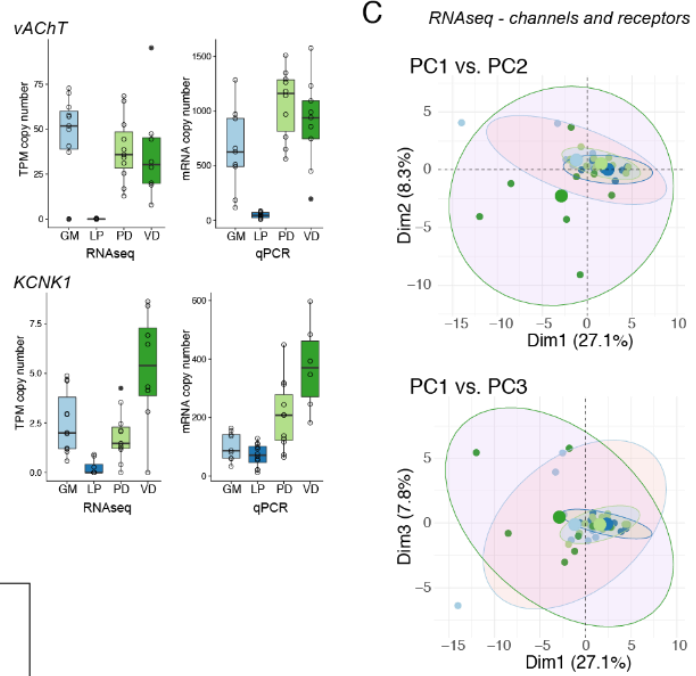

PC2 vs. PC3

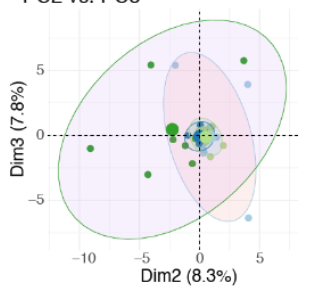

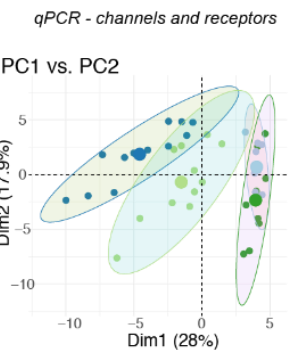

PC1 vs. PC3

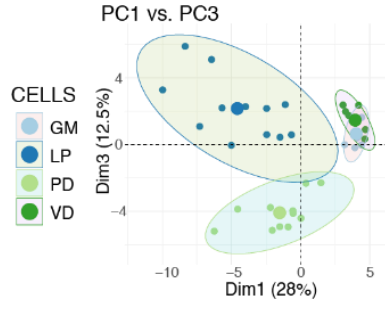

PC2 vs. PC3

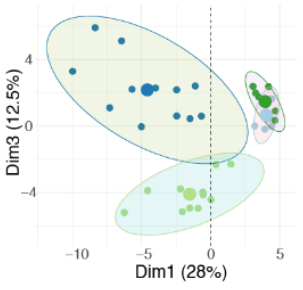

Comparison of expression levels and clustering between qRT-PCR and RNA-seq data. (A) Expression levels of 4 different genes (choline acetyltransferase [ChAT], vesicular acetylcholine transporter [vAChT], NMDA receptor subtype 2B [NMDA2B], and K+ two-pore-domain channel subfamily $\mathrm{K}$ member 1 [KCNK1]) between the RNA-seq and qRT-PCR datasets. Data shown are medians, quartiles, and each individual value from a given animal. Each individual data point is also represented as open circles. RNA-seq data are presented as TPM while qRT-PCR data as absolute copy number per cell. (B) Hierarchical clustering comparison between qRT-PCR (Top) and RNA-seq (Bottom) for the same 65 genes represented in the genes of interest pool shown in Fig. 4.1. Each 
cell type is color coded, and nodes are labeled with $\mathrm{AU}$ values as in previous

figures. (C) PCA for scRNA-seq versus qRT-PCR channel and receptor data.

Pairwise comparisons of PC1, PC2, and PC3 are shown in each panel as in Fig.

4.3. 


\section{Supplemental Figures}

Figure 4.S1 Abundance of ion channels, innexins, and NA/K ATPase contigs

Count numbers for selected voltage-gated ion channels from the RNA-seq data. The median counts for each of the voltage-gated channels used in the RT-PCR analysis was generated by pooling cell type. Innexins and the $\mathrm{Na}+\mathrm{K}+\mathrm{ATPase}$ are used as a reference of more highly abundant gene products.

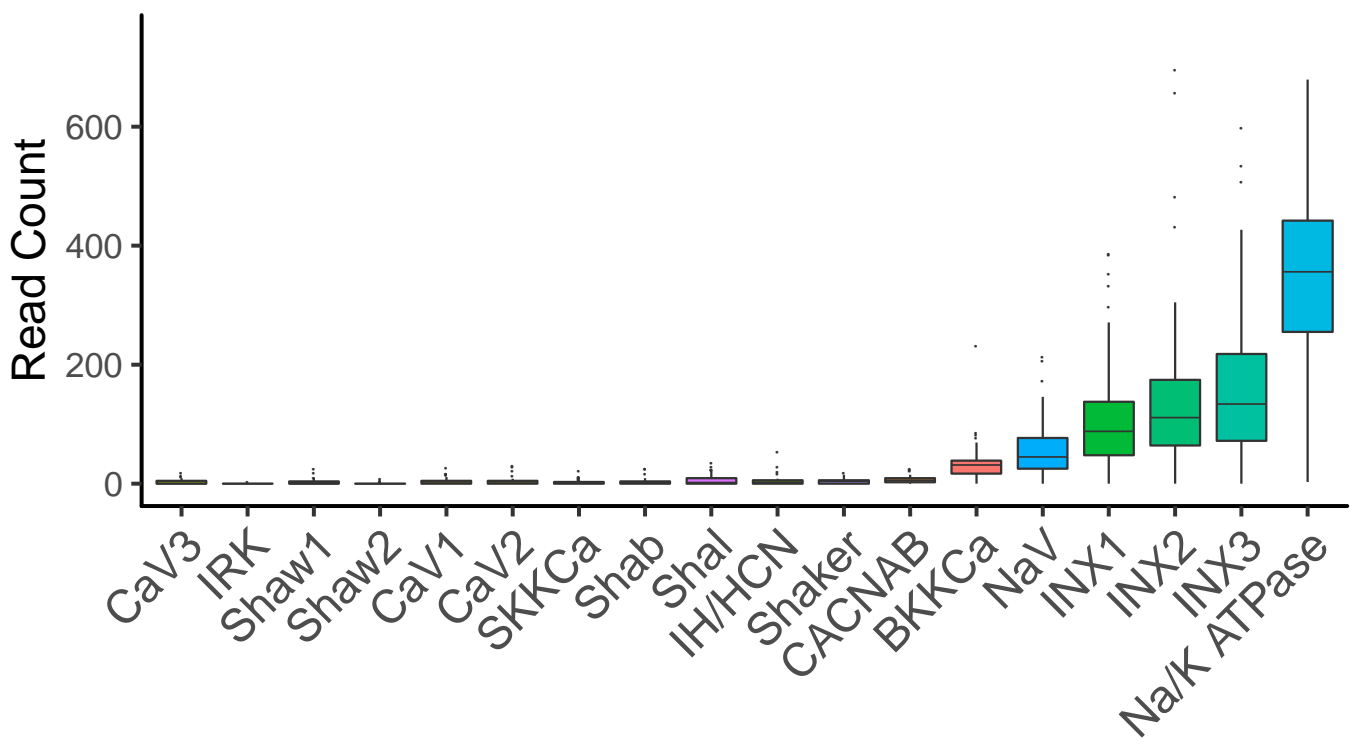




\section{Figure 4.S2 Effect of preamplification of quantitation cycle}

Comparison of expression levels of the same single cell samples $(\mathrm{N}=5)$ before (Unamplified) and after (Amplified) 14 cycles of preamplification of cDNA. Each sample represents a cDNA pool from a single identified neuron, half of which was preamplified and half remained unamplified. Data are shown as quantitation cycle (Cq) values. Statistics shown report values for Pearson's Correlation test.

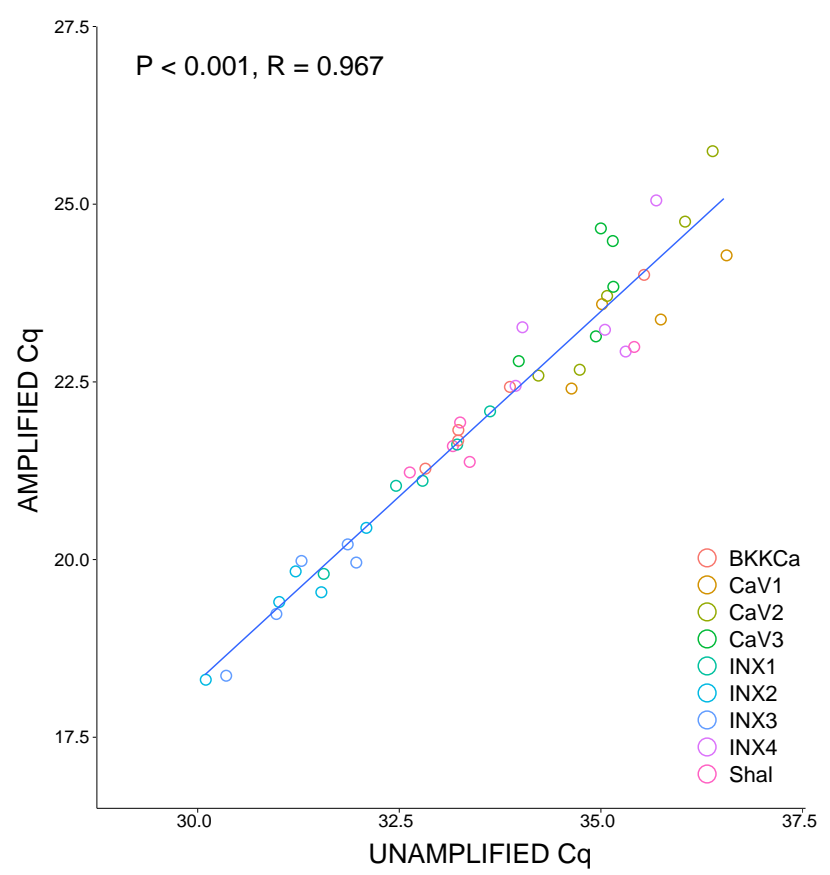


Table 4.S1: Top ten contributing genes or contigs to PCs1-3 for each

dataset.

\begin{tabular}{|c|c|c|c|c|c|c|c|}
\hline Dataset & Rank & PC1 & PC1 value & PC2 & PC2 value & PC3 & PC3 value \\
\hline$q R T P C R$ & 1 & $\mathrm{LCCH} 3 \mathrm{r}$ & 3.54 & $\mathrm{NaV}$ & 6.30 & GluCl & 9.12 \\
\hline$q R T P C R$ & 2 & mGABA3 & 3.10 & Shal & 5.65 & GABAB_R1 & 8.04 \\
\hline$q R T P C R$ & 3 & mGluR5 & 3.06 & NMDA_1A & 5.16 & vAChT & 7.56 \\
\hline$q R T P C R$ & 4 & CaV2 & 3.05 & KCNK1 & 4.95 & HisCL & 6.74 \\
\hline$q R T P C R$ & 5 & Shab & 3.02 & Shaker & 4.54 & ChAT & 6.57 \\
\hline$q R T P C R$ & 6 & KCNH3 & 2.97 & $\mathrm{IH}$ & 3.47 & $\mathrm{IH}$ & 6.16 \\
\hline$q R T P C R$ & 7 & DAR1A & 2.91 & KCNK2 & 3.45 & INX4 & 6.13 \\
\hline$q R T P C R$ & 8 & SKKCa & 2.87 & $\mathrm{BKKCa}$ & 3.10 & vGluT & 5.29 \\
\hline$q R T P C R$ & 9 & His_3r & 2.86 & Dopa_1Br & 3.06 & RDLr & 4.16 \\
\hline$q R T P C R$ & 10 & TRP_A_like & 2.82 & RDLr $r$ & 2.93 & CCAPr & 3.11 \\
\hline seq_h2k & 1 & c1318 & 0.10 & c4636 & 0.50 & c4191 & 0.59 \\
\hline seq_h2k & 2 & c724 & 0.10 & c8463 & 0.50 & c751 & 0.58 \\
\hline seq_h2k & 3 & c2022 & 0.10 & c28755 & 0.50 & c8533 & 0.49 \\
\hline seq_h2k & 4 & c1834 & 0.10 & c17319 & 0.50 & c953 & 0.46 \\
\hline seq_h2k & 5 & c718 & 0.10 & c10220 & 0.50 & c2665 & 0.46 \\
\hline seq_h2k & 6 & c2357 & 0.10 & c27163 & 0.49 & c2126 & 0.45 \\
\hline seq_h2k & 7 & c196 & 0.10 & c5528 & 0.49 & c2981 & 0.45 \\
\hline seq_h2k & 8 & c739 & 0.10 & c10716 & 0.49 & c3881 & 0.42 \\
\hline seq_h2k & 9 & c2301 & 0.10 & c9333 & 0.49 & c23433 & 0.41 \\
\hline seq_h2k & 10 & c1048 & 0.10 & c13463 & 0.49 & c1647 & 0.40 \\
\hline seq_hvg & 1 & c38450 & 0.50 & c18443 & 0.63 & c29394 & 0.80 \\
\hline seq_hvg & 2 & c5595 & 0.50 & c17911 & 0.63 & c23916 & 0.80 \\
\hline seq_hvg & 3 & c28755 & 0.50 & c13615 & 0.63 & c39794 & 0.80 \\
\hline seq_hvg & 4 & c11256 & 0.49 & c16416 & 0.63 & c24360 & 0.80 \\
\hline seq_hvg & 5 & c20433 & 0.49 & c17569 & 0.63 & c18403 & 0.79 \\
\hline seq_hvg & 6 & c39762 & 0.49 & c17622 & 0.63 & c7694 & 0.79 \\
\hline seq_hvg & 7 & c13489 & 0.49 & c18306 & 0.63 & c11984 & 0.79 \\
\hline seq_hvg & 8 & c19224 & 0.49 & c19165 & 0.63 & c16991 & 0.79 \\
\hline seq_hvg & 9 & c30088 & 0.49 & c19999 & 0.63 & c18899 & 0.79 \\
\hline seq_hvg & 10 & c4923 & 0.49 & c22142 & 0.63 & c25542 & 0.79 \\
\hline seq_DE0.2 & 1 & c5749 & 1.62 & c4517 & 3.20 & c13441 & 5.94 \\
\hline seq_DE0.2 & 2 & c1898 & 1.44 & c398 & 2.92 & c1058 & 4.27 \\
\hline seq_DE0.2 & 3 & c23967 & 1.43 & c878 & 2.92 & c31757 & 3.14 \\
\hline seq_DE0.2 & 4 & c5120 & 1.41 & c4945 & 2.87 & c9248 & 3.05 \\
\hline seq_DE0.2 & 5 & c1219 & 1.39 & c3559 & 2.69 & c2212 & 3.02 \\
\hline seq_DE0.2 & 6 & c8871 & 1.37 & c15559 & 2.64 & c4534 & 2.63 \\
\hline seq_DE0.2 & 7 & c972 & 1.35 & c8507 & 2.50 & c8114 & 2.61 \\
\hline seq_DE0.2 & 8 & c973 & 1.33 & c1151 & 2.15 & c10145 & 2.57 \\
\hline seq_DE0.2 & 9 & c12663 & 1.32 & c8323 & 2.06 & c2981 & 2.47 \\
\hline seq_DE0.2 & 10 & c910 & 1.30 & c1800 & 2.00 & c14660 & 2.35 \\
\hline seq_DE0.05 & 1 & c21272 & 4.69 & c4517 & 8.85 & c1058 & 13.16 \\
\hline seq_DE0.05 & 2 & c5716 & 4.21 & c16963 & 7.16 & c13441 & 11.60 \\
\hline seq_DE0.05 & 3 & c5120 & 4.14 & c3348 & 6.68 & c2212 & 10.64 \\
\hline seq_DE0.05 & 4 & c3737 & 3.77 & c1151 & 6.05 & c2586 & 7.17 \\
\hline seq_DE0.05 & 5 & c8114 & 3.72 & c14320 & 5.14 & c5845 & 5.83 \\
\hline seq_DE0.05 & 6 & c16240 & 3.59 & c5222 & 4.93 & c14320 & 4.87 \\
\hline seq_DE0.05 & 7 & c2796 & 3.56 & c8323 & 4.57 & c4997 & 4.10 \\
\hline seq_DE0.05 & 8 & c1713 & 3.43 & c5067 & 4.35 & c14660 & 4.06 \\
\hline seq_DE0.05 & 9 & $c 49$ & 3.38 & c1324 & 4.12 & c3348 & 4.00 \\
\hline seq_DE0.05 & 10 & c3716 & 3.23 & c24846 & 4.08 & c4534 & 3.86 \\
\hline
\end{tabular}


Table 4.S2: Gene Ontology Enrichment analysis of Molecular Function for H2K RNAseq data.

\begin{tabular}{|c|c|c|}
\hline GO term: molecular function & $\begin{array}{c}\text { Fold } \\
\text { Enrichment }\end{array}$ & $\begin{array}{c}\text { FDR } \\
\text { p-value }\end{array}$ \\
\hline proton-transporting ATP synthase activity, rotational mechanism (GO:0046933) & $8.08+$ & $2.88 \mathrm{E}-04$ \\
\hline clathrin binding (GO:0030276) & $6.82+$ & $4.51 \mathrm{E}-03$ \\
\hline ubiquitin conjugating enzyme activity (GO:0061631) & $4.6+$ & 1.59E-02 \\
\hline intramolecular oxidoreductase activity (GO:0016860) & $4.32+$ & 2.22E-02 \\
\hline structural constituent of ribosome (GO:0003735) & $4.02+$ & $4.61 \mathrm{E}-11$ \\
\hline proton-transporting ATPase activity, rotational mechanism (GO:0046961) & $3.84+$ & 4.35E-02 \\
\hline structural constituent of cytoskeleton (GO:0005200) & $3.84+$ & 4.30E-02 \\
\hline unfolded protein binding (GO:0051082) & $3.8+$ & 4.00E-05 \\
\hline heat shock protein binding (GO:0031072) & $3.73+$ & $5.01 \mathrm{E}-02$ \\
\hline mRNA 3'-UTR binding (GO:0003730) & $3.73+$ & 4.96E-02 \\
\hline cell adhesion molecule binding (GO:0050839) & $3.57+$ & 3.89E-02 \\
\hline translation factor activity, RNA binding (GO:0008135) & $3.27+$ & $4.57 \mathrm{E}-03$ \\
\hline electron transfer activity (GO:0009055) & $3.07+$ & 1.79E-02 \\
\hline GTPase activity (GO:0003924) & $3.07+$ & $6.09 \mathrm{E}-05$ \\
\hline GTP binding (GO:0005525) & $2.97+$ & $6.78 \mathrm{E}-05$ \\
\hline actin binding (GO:0003779) & $2.95+$ & 4.42E-04 \\
\hline kinase binding (GO:0019900) & $2.85+$ & $7.78 \mathrm{E}-03$ \\
\hline microtubule binding (GO:0008017) & $2.75+$ & $3.98 \mathrm{E}-03$ \\
\hline phospholipid binding (GO:0005543) & $2.56+$ & $3.16 \mathrm{E}-02$ \\
\hline calcium ion binding (GO:0005509) & $2.4+$ & $1.60 \mathrm{E}-03$ \\
\hline protein-containing complex binding (GO:0044877) & $2.39+$ & 7.29E-04 \\
\hline ATP binding (GO:0005524) & $2.31+$ & $1.36 \mathrm{E}-10$ \\
\hline protein serine/threonine kinase activity (GO:0004674) & $2.17+$ & $1.31 \mathrm{E}-02$ \\
\hline enzyme regulator activity (GO:0030234) & $1.83+$ & $1.40 \mathrm{E}-02$ \\
\hline DNA-binding transcription factor activity (GO:0003700) & $0.41-$ & 1.79E-02 \\
\hline serine-type endopeptidase activity (GO:0004252) & $0.1-$ & $1.90 \mathrm{E}-03$ \\
\hline
\end{tabular}


Table 4.S3: Gene Ontology Enrichment analysis of Biological Process for H2K RNAseq data.

\begin{tabular}{|c|c|c|}
\hline GO term: biological process & $\begin{array}{c}\text { Fold } \\
\text { Enrichment }\end{array}$ & $\begin{array}{c}\text { FDR } \\
\text { p-value }\end{array}$ \\
\hline regulation of short-term neuronal synaptic plasticity (GO:0048172) & $10.96+$ & $6.64 \mathrm{E}-03$ \\
\hline positive regulation of neuron remodeling (GO:1904801) & $10.63+$ & 7.39E-05 \\
\hline substrate adhesion-dependent cell spreading (GO:0034446) & $10.23+$ & 2.68E-03 \\
\hline actin filament polymerization (GO:0030041) & $9.59+$ & $9.52 \mathrm{E}-03$ \\
\hline clathrin-dependent synaptic vesicle endocytosis (GO:0150007) & $8.77+$ & 3.43E-02 \\
\hline protein N-linked glycosylation via asparagine (GO:0018279) & $8.77+$ & $3.42 \mathrm{E}-02$ \\
\hline gluconeogenesis (GO:0006094) & $8.53+$ & $1.31 \mathrm{E}-02$ \\
\hline dorsal closure, spreading of leading edge cells (GO:0007395) & $8.53+$ & $1.31 \mathrm{E}-02$ \\
\hline morphogenesis of larval imaginal disc epithelium (GO:0016335) & $7.67+$ & 4.63E-02 \\
\hline retrograde axonal transport (GO:0008090) & $7.67+$ & 1.79E-02 \\
\hline ATP synthesis coupled proton transport (GO:0015986) & $7.03+$ & 1.14E-04 \\
\hline vesicle transport along microtubule (GO:0047496) & $6.98+$ & $2.40 \mathrm{E}-02$ \\
\hline axonal transport of mitochondrion (GO:0019896) & $6.98+$ & 2.39E-02 \\
\hline anterograde axonal transport (GO:0008089) & $6.98+$ & 2.39E-02 \\
\hline cellular response to metal ion (GO:0071248) & $6.39+$ & 3.07E-02 \\
\hline axonal fasciculation (GO:0007413) & $6.14+$ & 3.23E-03 \\
\hline female germ-line stem cell asymmetric division (GO:0048132) & $5.97+$ & $7.80 \mathrm{E}-03$ \\
\hline regulation of reactive oxygen species metabolic process (GO:2000377) & $5.9+$ & 3.80E-02 \\
\hline actin nucleation (GO:0045010) & $5.48+$ & 4.75E-02 \\
\hline positive regulation of photoreceptor cell differentiation (GO:0046534) & $5.48+$ & 4.74E-02 \\
\hline sevenless signaling pathway (GO:0045500) & $5.42+$ & $2.40 \mathrm{E}-02$ \\
\hline ovarian follicle cell stalk formation (GO:0030713) & $5.42+$ & 2.39E-02 \\
\hline synaptic vesicle priming (GO:0016082) & $5.42+$ & 2.39E-02 \\
\hline flight behavior (GO:0007629) & $5.42+$ & 2.39E-02 \\
\hline positive regulation of endocytosis (GO:0045807) & $5.42+$ & 3.15E-04 \\
\hline positive regulation of lipid localization (GO:1905954) & $5.42+$ & 2.38E-02 \\
\hline positive regulation of canonical Wnt signaling pathway (GO:0090263) & $5.39+$ & $1.58 \mathrm{E}-04$ \\
\hline ribosomal large subunit assembly (GO:0000027) & $5.37+$ & 1.19E-02 \\
\hline positive regulation of smoothened signaling pathway (GO:0045880) & $5.31+$ & 3.15E-03 \\
\hline
\end{tabular}


positive regulation of protein modification by small protein conjugation or removal (GO:1903322)

$3.74+\quad 1.15 \mathrm{E}-02$ 


\begin{tabular}{|c|c|c|}
\hline negative regulation of cytoskeleton organization (GO:0051494) & $3.72+$ & $3.20 \mathrm{E}-02$ \\
\hline negative regulation of smoothened signaling pathway (GO:0045879) & $3.72+$ & $3.20 \mathrm{E}-02$ \\
\hline mitochondrial ATP synthesis coupled electron transport (GO:0042775) & $3.65+$ & $1.75 \mathrm{E}-04$ \\
\hline regulation of peptide secretion (GO:0002791) & $3.64+$ & $2.21 \mathrm{E}-02$ \\
\hline behavioral response to ethanol (GO:0048149) & $3.63+$ & $3.40 \mathrm{E}-03$ \\
\hline regulation of chemotaxis (GO:0050920) & $3.61+$ & 3.63E-02 \\
\hline response to unfolded protein (GO:0006986) & $3.61+$ & $3.62 \mathrm{E}-02$ \\
\hline positive regulation of cell size (GO:0045793) & $3.57+$ & $1.48 \mathrm{E}-02$ \\
\hline tight junction organization (GO:0120193) & $3.54+$ & $2.50 \mathrm{E}-02$ \\
\hline apical junction assembly (GO:0043297) & $3.52+$ & $1.02 \mathrm{E}-02$ \\
\hline cytosolic transport (GO:0016482) & $3.5+$ & 4.39E-03 \\
\hline cytokinetic process (GO:0032506) & $3.49+$ & 1.69E-02 \\
\hline regulation of axonogenesis (GO:0050770) & $3.47+$ & 3.00E-03 \\
\hline negative regulation of protein phosphorylation (GO:0001933) & $3.44+$ & 2.05E-03 \\
\hline positive regulation of cell morphogenesis involved in differentiation (GO:0010770) & $3.41+$ & 4.69E-02 \\
\hline establishment or maintenance of apical/basal cell polarity (GO:0035088) & $3.37+$ & $3.20 \mathrm{E}-02$ \\
\hline regulation of multicellular organism growth (GO:0040014) & $3.34+$ & $2.20 \mathrm{E}-02$ \\
\hline morphogenesis of follicular epithelium (GO:0016333) & $3.31+$ & 1.47E-02 \\
\hline translational initiation (GO:0006413) & $3.25+$ & 1.65E-02 \\
\hline regulation of protein stability (GO:0031647) & $3.25+$ & 1.65E-02 \\
\hline neuromuscular synaptic transmission (GO:0007274) & $3.23+$ & 1.13E-02 \\
\hline autophagy (GO:0006914) & $3.23+$ & 4.15E-04 \\
\hline imaginal disc-derived wing margin morphogenesis (GO:0008587) & $3.19+$ & 1.86E-02 \\
\hline mitotic cytokinesis (GO:0000281) & $3.16+$ & 6.07E-03 \\
\hline long-term memory (GO:0007616) & $3.15+$ & 4.15E-03 \\
\hline germ-line stem cell population maintenance (GO:0030718) & $3.15+$ & $2.84 \mathrm{E}-03$ \\
\hline asymmetric neuroblast division (GO:0055059) & $3.14+$ & $4.50 \mathrm{E}-02$ \\
\hline cell redox homeostasis (GO:0045454) & $3.13+$ & 3.09E-02 \\
\hline response to growth factor (GO:0070848) & $3.13+$ & $3.08 \mathrm{E}-02$ \\
\hline synaptic target recognition (GO:0008039) & $3.13+$ & 3.07E-02 \\
\hline amino acid transport (GO:0006865) & $3.01+$ & 3.76E-02 \\
\hline axon guidance (GO:0007411) & $2.94+$ & 1.77E-08 \\
\hline positive regulation of locomotion (GO:0040017) & $2.63+$ & 4.08E-02 \\
\hline negative regulation of neurogenesis (GO:0050768) & $2.63+$ & 3.09E-02 \\
\hline
\end{tabular}


Table 4.S4: Gene Ontology Enrichment analysis of Molecular Function for HVG RNAseq data.

\begin{tabular}{lcc}
\hline & $\begin{array}{c}\text { Fold } \\
\text { GO term: molecular function }\end{array}$ & FDR \\
\hline ATP binding (GO:0005524) & $3.1+$ & $1.72 \mathrm{E}-03$ \\
transferase activity (GO:0016740) & $2+$ & $2.38 \mathrm{E}-02$ \\
\hline
\end{tabular}

Table 4.S5: Gene Ontology Enrichment analysis of Biological Process for HVG RNAseq data.

\begin{tabular}{lcc}
\hline GO term: molecular function & $\begin{array}{c}\text { Fold } \\
\text { Enrichment }\end{array}$ & $\begin{array}{c}\text { FDR } \\
\text { p-value }\end{array}$ \\
\hline regulation of protein localization to plasma membrane (GO:1903076) & $35.33+$ & $4.79 \mathrm{E}-02$ \\
chromatin silencing (GO:0006342) & $7.69+$ & $3.12 \mathrm{E}-02$ \\
nucleic acid metabolic process (GO:0090304) & $2.17+$ & $3.74 \mathrm{E}-02$ \\
macromolecule modification (GO:0043412) & $2.08+$ & $3.91 \mathrm{E}-02$ \\
cellular macromolecule metabolic process (GO:0044260) & $1.84+$ & $2.22 \mathrm{E}-02$ \\
\hline
\end{tabular}




\section{Table 4.S6: Gene Ontology Enrichment analysis of Molecular Function for DE0.2 RNAseq data.}

\begin{tabular}{|c|c|c|}
\hline GO term: molecular function & $\begin{array}{c}\text { Fold } \\
\text { Enrichment }\end{array}$ & $\begin{array}{c}\text { raw } \\
\text { p-value }\end{array}$ \\
\hline choline:sodium symporter activity (GO:0005307) & $>100+$ & $7.51 \mathrm{E}-03$ \\
\hline acetylcholine transmembrane transporter activity (GO:0005277) & $>100+$ & 7.51E-03 \\
\hline dihydroorotase activity (GO:0004151) & $>100+$ & 7.51E-03 \\
\hline choline O-acetyltransferase activity (GO:0004102) & $>100+$ & $7.51 \mathrm{E}-03$ \\
\hline carboxyl- or carbamoyltransferase activity (GO:0016743) & $>100+$ & 7.51E-03 \\
\hline carbamoyl-phosphate synthase (glutamine-hydrolyzing) activity (GO:0004088) & $>100+$ & $7.51 \mathrm{E}-03$ \\
\hline aspartate carbamoyltransferase activity (GO:0004070) & $>100+$ & $7.51 \mathrm{E}-03$ \\
\hline very-long-chain-acyl-CoA dehydrogenase activity (GO:0017099) & $>100+$ & 7.51E-03 \\
\hline latrotoxin receptor activity (GO:0016524) & $>100+$ & $7.51 \mathrm{E}-03$ \\
\hline glutamine binding (GO:0070406) & $>100+$ & $7.51 \mathrm{E}-03$ \\
\hline L-iduronidase activity (GO:0003940) & $>100+$ & $7.51 \mathrm{E}-03$ \\
\hline choline binding (GO:0033265) & $>100+$ & $7.51 \mathrm{E}-03$ \\
\hline myosin II light chain binding (GO:0032033) & $>100+$ & $1.12 \mathrm{E}-02$ \\
\hline kinetochore binding (GO:0043515) & $>100+$ & $1.12 \mathrm{E}-02$ \\
\hline GABA-gated chloride ion channel activity (GO:0022851) & $88.25+$ & $1.50 \mathrm{E}-02$ \\
\hline receptor antagonist activity (GO:0048019) & $88.25+$ & $1.50 \mathrm{E}-02$ \\
\hline GABA-A receptor activity (GO:0004890) & $66.19+$ & 1.87E-02 \\
\hline smoothened binding (GO:0005119) & $66.19+$ & 1.87E-02 \\
\hline patched binding (GO:0005113) & $66.19+$ & 1.87E-02 \\
\hline histone demethylase activity (H3-K4 specific) (GO:0032453) & $66.19+$ & 1.87E-02 \\
\hline kinesin binding (GO:0019894) & $58.83+$ & 7.47E-04 \\
\hline histone demethylase activity (H3-K36 specific) (GO:0051864) & $52.95+$ & 2.24E-02 \\
\hline morphogen activity (GO:0016015) & $52.95+$ & 2.24E-02 \\
\hline MAP-kinase scaffold activity (GO:0005078) & $52.95+$ & 2.24E-02 \\
\hline extracellular matrix binding (GO:0050840) & $52.95+$ & 2.24E-02 \\
\hline axon guidance receptor activity (GO:0008046) & $44.12+$ & $2.61 \mathrm{E}-02$ \\
\hline protein kinase $\mathrm{C}$ binding (GO:0005080) & $44.12+$ & $2.61 \mathrm{E}-02$ \\
\hline RNA polymerase II activity (GO:0001055) & $29.42+$ & $3.70 \mathrm{E}-02$ \\
\hline epidermal growth factor receptor binding (GO:0005154) & $29.42+$ & $3.70 \mathrm{E}-02$ \\
\hline phosphatidylserine binding (GO:0001786) & $29.42+$ & $3.70 \mathrm{E}-02$ \\
\hline
\end{tabular}




\begin{tabular}{lcc} 
cell-cell adhesion mediator activity (GO:0098632) & $24.07+$ & $4.42 \mathrm{E}-02$ \\
fatty-acyl-CoA binding (GO:0000062) & $24.07+$ & $4.42 \mathrm{E}-02$ \\
microtubule plus-end binding (GO:0051010) & $22.06+$ & $4.78 \mathrm{E}-02$ \\
calcium-dependent phospholipid binding (GO:0005544) & $22.06+$ & $4.78 \mathrm{E}-02$ \\
amino acid transmembrane transporter activity (GO:0015171) & $9.46+$ & $2.01 \mathrm{E}-02$ \\
flavin adenine dinucleotide binding (GO:0050660) & $7.79+$ & $2.85 \mathrm{E}-02$ \\
GTP binding (GO:0005525) & $5.12+$ & $2.15 \mathrm{E}-02$ \\
\hline
\end{tabular}


Table 4.S7: Target primer and probe sequences for qRT-PCR Multiplex

assays.

\section{Each box represents a group of four to five genes that were combined into a}

\section{single multiplex reaction.}

\begin{tabular}{|c|c|c|c|}
\hline Gene & $\begin{array}{l}\text { Accessio } \\
n \#\end{array}$ & Forward / Reverse Primer 5'-3' sequence & Probe 5'-3' sequence \\
\hline HTR1A & KU710381.1 & $\begin{array}{l}\text { AACCGCTGTGGTAGTTTCCA / } \\
\text { TGCTCGTTAACCCGGACTAAG }\end{array}$ & AGCGCCTTTATTTGGCTGGAAGGA \\
\hline HTR2 & KU710380.1 & $\begin{array}{l}\text { TCCGCCTCCATCAAGTTTGT / } \\
\text { GCACGTTGGCGATGAAGAAC }\end{array}$ & TCATCGAAGAGACACGGGAGGACC \\
\hline HTR7 & KU710379.1 & $\begin{array}{l}\text { ACGGCGATGGCTCCATCTG / } \\
\text { CGGTGAGCGGGATGTAGAAG }\end{array}$ & $\begin{array}{c}\text { TGAGGTGTGCAACAACTTCTGGTAC } \\
\mathrm{C}\end{array}$ \\
\hline DAR2 & KU710378.1 & $\begin{array}{l}\text { GAAGCCGAAAGTGAGTGAGATCA / } \\
\text { TCCGAACTAAGCGCTGTTTC }\end{array}$ & $\underset{A}{\text { TGTGATCGAGAATGTGACACAGACG }}$ \\
\hline DAR1A & KU710377.1 & $\begin{array}{c}\text { GGCGCCTGTCCATTCACT / } \\
\text { CGCGGTAGATGCGGAAGTAAG }\end{array}$ & $\begin{array}{c}\text { АCCTTGTGTTCTCCTCTACTATCAG } \\
\text { СTTCT }\end{array}$ \\
\hline $\begin{array}{l}\text { GABAB- } \\
R 1\end{array}$ & KU986868.1 & $\begin{array}{l}\text { TCGTCTCGTTTGCCATCATC / } \\
\text { GGTGCCGAACCTCAATGATC }\end{array}$ & TCTGCTGCTTCCTCTCCATGGCT \\
\hline $\mathrm{LCCH} 3 \mathrm{r}$ & KU986871.1 & $\begin{array}{l}\text { TGACGGCTCCATCACCTATGG / } \\
\text { TTGGGTGTCGAGTGGATAGTAG }\end{array}$ & TTCACCACTACGTTGGCCTGCAT \\
\hline$R D L r$ & KU986872.1 & $\begin{array}{l}\text { TGGTGTTTGCCTCGCTTCTAG / } \\
\text { TCCGCTGTTCTGCTAACTTC }\end{array}$ & AATACGCCGCGGTGGGCTACAT \\
\hline GluCl & KX059698.1 & $\begin{array}{l}\text { ACGGAGGATCTGGTGTTTCTG / } \\
\text { ACCCGTGTTGGTCTTGCTGTT }\end{array}$ & TACAGGTGACCAAGAACCTTCACC \\
\hline NALCN & KU681457.1 & $\begin{array}{l}\text { TCGCTTCCACGGTGTACATTC / } \\
\text { GCGGTGCCTTTGTTCTCAG }\end{array}$ & TCTTCGTCTTCCTTGGCTGCATGA \\
\hline CACNAB & $\begin{array}{l}\text { GEFB01006 } \\
512\end{array}$ & $\begin{array}{l}\text { GCAGCTGGCCAAGACTTCTTT / } \\
\text { AGACGCTGCAATACCTTAGGA }\end{array}$ & AGCGCCCATCCTCGTGTACCTTAAG \\
\hline IRK & KU681451.1 & $\begin{array}{l}\text { TACAGTGGCGTTGGACTCTAC / } \\
\text { TCCACCACACCAAGGCAAATAG }\end{array}$ & $\begin{array}{c}\text { TCGTGTTCGCTATGTCATTCATCAG } \\
\text { C }\end{array}$ \\
\hline IH & DQ103257.3 & $\begin{array}{l}\text { TCGGTGCCACTAGACTACATC / } \\
\text { GACCCGCGTGGAGAATCTG }\end{array}$ & $\begin{array}{c}\text { TCCTCATCTTCAACCAGGACTTCAG } \\
\text { C }\end{array}$ \\
\hline$S K K C a$ & KU710383.1 & $\begin{array}{l}\text { GCATCGGAGCATTGAACAGAA / } \\
\text { GCCCGGACAGATAGTCATCAG }\end{array}$ & $\begin{array}{c}\text { CAACTTCAACACTCGGTTTGTCCTC } \\
\text { AA }\end{array}$ \\
\hline$I N X 1$ & JQ994479.1 & $\begin{array}{l}\text { TGGAGCGTCATGATGCATTC / } \\
\text { GAGCAGGATGGCAAGGATCAC }\end{array}$ & $\begin{array}{c}\text { TGCTGCCTCTCAACATTCTTAACGA } \\
\text { A }\end{array}$ \\
\hline INX2 & JQ994480.1 & $\begin{array}{l}\text { GGCTGTGGTGTCTGGTGTAG / } \\
\text { GCGAGAGCGTGTCCTTAACAG }\end{array}$ & CTGCTGTACCGCCTCGCCACTTT \\
\hline INX3 & JQ994481.1 & $\begin{array}{l}\text { TGTCGGCCCTAGTGAAAGAG / } \\
\text { GGTACCGTGGGATGTAGAACA }\end{array}$ & TGACGAGATTGTGTACCACGCTTAC \\
\hline INX4 & KJ642222.1 & $\begin{array}{l}\text { CTGGCGTTCAGCCTCATTGTC / } \\
\text { CACGTCCTCTGGGATCTCCTTAG }\end{array}$ & CACGCGTCAGTATGTCGGGAACC \\
\hline INX5 & KJ817410.1 & $\begin{array}{l}\text { TGCCTTCCCTGCTGGATAA / } \\
\text { GCGTCACCCATTGGTAGTAAC }\end{array}$ & AGGTGGCTCATCCAGGCATCGGT \\
\hline Shaw1 & KU681456.1 & $\begin{array}{l}\text { CGCGTCACTCCTCAGGACTT / } \\
\text { CCCAGCACCAGGAAGAACAC }\end{array}$ & TGATACAGACTTTCCGTGCATCCGC \\
\hline Shaw2 & KU681455.1 & $\begin{array}{l}\text { GAACGCCATCAAGCACTATCATC / } \\
\text { ATGGCGCCCGACAGCTTAG }\end{array}$ & TGGCTTGAAGGACGGTCTCACA \\
\hline $\mathrm{NaV}$ & EF089568.2 & $\begin{array}{l}\text { TCAACGGGAGGTACCATAAGTG / } \\
\text { TCGCTGTTCACCCAAGAGTAG }\end{array}$ & CGGAGGGATTGAAGCTCAACGCA \\
\hline Shaker & FJ263946.1 & $\begin{array}{l}\text { GAGGCTCAGAAGACCAGTCAAC / } \\
\text { TGGCGATATCACCGAGCTCAT }\end{array}$ & CACTCGATGTCTTCGCGGAGGAGAT \\
\hline Shab & DQ103255.1 & $\begin{array}{l}\text { GAGCCGGACAGACAGGAAC / } \\
\text { TGCGCCTCCTTCTGTAGTC }\end{array}$ & AAGAACCACGAACACCACATGGGTC \\
\hline CaV1 & JN809809.1 & $\begin{array}{l}\text { CCAGGCCTTCTACTGGCTCATT / } \\
\text { GCTGGCGATAGTGCTCACTG }\end{array}$ & TGTGCTCGTCTTCCTCAACACGG \\
\hline CaV2 & JN809808.1 & $\begin{array}{l}\text { ATCCGGCGGACAGTAAAGC / } \\
\text { GTTCGGCAGCAACACAAAC }\end{array}$ & $\begin{array}{c}\text { TGGTTCTACTGGTTCGTCATCATAC } \\
\text { TTGT }\end{array}$ \\
\hline CaV3 & JN809810.1 & $\begin{array}{l}\text { TGGCTGCCACCGATACTTC / } \\
\text { CAGCACAATGCCCACAACTG }\end{array}$ & $\begin{array}{c}\text { CAGGACAGAGATGGAACCAGTTGG } \\
\text { A }\end{array}$ \\
\hline Shal & DQ103254.1 & $\begin{array}{l}\text { GACACCACCTTCACCTCCATTC / } \\
\text { GAACCATGTCGCCGTATCCTA }\end{array}$ & CGGCGTTTTGGTACACCATTGTCAC \\
\hline$B K K C a$ & DQ103256.4 & $\begin{array}{l}\text { GCTCAAACTCGGCTTCATTG / } \\
\text { CTGCGTGTCTGGAGAAGTTT }\end{array}$ & AGAATCCCGGCGCTAAACATGACT \\
\hline mGluR1 & KU986879.1 & $\begin{array}{l}\text { GCATCGTGTGGCTCATCTTTG / } \\
\text { GGCCAACGTGACCACTCTAAT }\end{array}$ & ACCTGTCAGCGGGAGTCACTGATG \\
\hline mGluR2 & KU986880.1 & $\begin{array}{c}\text { TCCGCAGGTGAGTTTCTTC / } \\
\text { CCATGGCTTTCACTTGGTAATGG }\end{array}$ & CCACGTCGCCTGAACTGAGTAACA \\
\hline mGluR4 & KU986882.1 & $\begin{array}{l}\text { GCGCGTTGATTCCGGTACT / } \\
\text { CCACTCATCGTCCTCAACTTC }\end{array}$ & AAGTCTTCCCGCTGGACTACGAAC \\
\hline mGluR5 & KU986883.1 & $\begin{array}{l}\text { GCCTGTCCTTTGCCATGATC / } \\
\text { TGCGCATCGTGATCTTCTTG }\end{array}$ & CGCTTGTCACCAAGACCAACCGC \\
\hline mGluR7 & KU986884.1 & $\begin{array}{l}\text { ACCGCGCTCGCAGATTGTC / } \\
\text { TGGCTGGCGTTTCCACTATC }\end{array}$ & TTCTGGACTGGTGAGTGTTCAGCT \\
\hline
\end{tabular}




\begin{tabular}{|c|c|c|c|}
\hline$m A C H r A$ & KX021822.1 & $\begin{array}{l}\text { GGTGTCGATGCCTTTGTTCAC / } \\
\text { GCCAGCCAGGTGTCACATAT }\end{array}$ & TGTACACGCTGATGGGATACTGGC \\
\hline$m A C H r B$ & KX021821.1 & $\begin{array}{l}\text { GCGGACCATCTCCATCATTC / } \\
\text { TGGTGCACTGACAGAAGCT }\end{array}$ & CCTTTGTGGCCTGTTGGACGCC \\
\hline$m G A B A 2$ & KU986869.1 & $\begin{array}{l}\text { TGCCTACAAAGGTCTGCTAATGG / } \\
\text { TCGTTCAAGGCGGGTATGTT }\end{array}$ & TGGTTGTTTCCTGGCCTGGGAAA \\
\hline$m G A B A 3$ & KU986870.1 & $\begin{array}{l}\text { CGATCCCATGACGAGACACAT / } \\
\text { TCCACCTGTGGCTGATAGAC }\end{array}$ & CCTCACCTTAGAAGTGTCAGCCGA \\
\hline mGluR3 & KU986881.1 & $\begin{array}{l}\text { CACCGTGTATGCCGTCAAGAC / } \\
\text { CCCGGTGCCGAAGTAGATG }\end{array}$ & $\begin{array}{c}\text { CCCCGAGAATTTCAACGAGAGCAAG } \\
\mathrm{T}\end{array}$ \\
\hline TRP-M3 & KX037433.1 & $\begin{array}{l}\text { CCGCACCATCTACGAGAAC / } \\
\text { TGCTGGCCTGGAAGATGT }\end{array}$ & TGCTCAAGTCTCCTCGTCTTCACC \\
\hline $\begin{array}{l}\text { TRP-A- } \\
\text { like }\end{array}$ & KX037434.1 & $\begin{array}{l}\text { TCGCGACCTTCCTCAAATTC / } \\
\text { CGGTACCTGAGTCCTCAACAC }\end{array}$ & $\begin{array}{c}\text { CACGGTCTTCTTACTCTTCCTCATC } \\
\text { GC }\end{array}$ \\
\hline TRP-A1 & KX037435.1 & $\begin{array}{l}\text { CTGCCAAGTACGGTCGTTACAAC / } \\
\text { CCCTCGTCATTGCACTCGTTA }\end{array}$ & ACGTCAGCTTGTGGAGTCTCTGAA \\
\hline TRP-M1 & KX037436.1 & $\begin{array}{l}\text { GAGGGCGGACCTCAAACTATC / } \\
\text { TGTCGGCTGCTCTTCCTGTT }\end{array}$ & CGTCAGGTGCTGGAGTATGTCACTG \\
\hline $\begin{array}{l}\text { TRP-M- } \\
\text { like }\end{array}$ & KX037437.1 & $\begin{array}{l}\text { GACGGGACGCAGATCCTCTT / } \\
\text { GAGTGCTTGGGCTGTTAGGT }\end{array}$ & ACGGTATACGGTTGGCTATTTCCCA \\
\hline Dopa-1Br & KU710376.1 & $\begin{array}{l}\text { CGCAAGATTGGCAACCTCTTC / } \\
\text { ACGAGGGCAAAGCTCATCAC }\end{array}$ & TGTCCTTGGCTATCGCTGACCTCTT \\
\hline $\begin{array}{l}5 H T R- \\
1 B r\end{array}$ & KU710382.1 & $\begin{array}{l}\text { TGACGCAGGTGGACTACATTC / } \\
\text { GGAACGACACCACCCAGATC }\end{array}$ & ACCGATCGCCGCGTCGCATC \\
\hline His-1r & KU716100.1 & $\begin{array}{l}\text { TGCCTGCCAGAGTAACCTTAG / } \\
\text { GACAGGTGGGAGGGATTTCTG }\end{array}$ & $\begin{array}{c}\text { CATCACTTCTCACTCTGTCAACCCA } \\
\text { ACA }\end{array}$ \\
\hline His-2r & KU716101.1 & $\begin{array}{l}\text { CCGCCACAGTCTCAAGGTAATC / } \\
\text { GCGTAGGTCATGGAACTCTCATC }\end{array}$ & ACGGTAGTCTACTTCCACGTCACA \\
\hline His-3r & KU716102.1 & $\begin{array}{l}\text { ATCCGCCGCAACAAAGCAT / } \\
\text { GAGAGCGAAGGAGGTTGGAA }\end{array}$ & $\begin{array}{l}\text { TGATGGTGGATCGAGTCTCAAGATA } \\
\text { TGTAT }\end{array}$ \\
\hline $\begin{array}{l}\text { kainate- } \\
1 A\end{array}$ & KX016772.1 & $\begin{array}{l}\text { CAGGTCGGAGTGCAGTAAAGAC / } \\
\text { GCCACCAGTCAGGATGTAGAAG }\end{array}$ & CGATGACCACCCAGACGAGTGC \\
\hline $\begin{array}{l}\text { kainate- } \\
1 B\end{array}$ & KX016773.1 & $\begin{array}{l}\text { TGAGCAGAACGAGATCGAGTATG / } \\
\text { CGCCACATGTTCTGATACGTC }\end{array}$ & AGGGCGGGTCTACCATGGCCTT \\
\hline $\begin{array}{l}\text { kainate- } \\
2 A\end{array}$ & KX016774.1 & $\begin{array}{l}\text { CGCATGGAGTCACCTATTGAGA / } \\
\text { AGGCGAAAGTGGTGCCAGTTG }\end{array}$ & TGAGGACCTTGCTAACCAGGACAA \\
\hline $\begin{array}{l}\text { kainate- } \\
2 B\end{array}$ & KX016775.1 & $\begin{array}{l}\text { GCACGGCAAGTTTGACAAGAAG / } \\
\text { TGCTCCCTCTCGTAAGTGATG }\end{array}$ & AACGGCATGATTGGGCAGCTGTT \\
\hline $\begin{array}{l}\text { kainate- } \\
2 C\end{array}$ & KX016776.1 & $\begin{array}{l}\text { GGCTTGGTCAGGGAACTCAAG / } \\
\text { GCTCTCCCTCGCGTAGTTG }\end{array}$ & $\begin{array}{c}\text { TGATCTAGCGGTGGGTTCTATGACT } \\
\text { A }\end{array}$ \\
\hline $\begin{array}{l}\text { NMDA- } \\
1 A\end{array}$ & KX016782.1 & $\begin{array}{l}\text { GCCGTCAAATCAGGGAGGTT / } \\
\text { ACCGGCGGTTACCAGTTCAC }\end{array}$ & $\begin{array}{c}\text { AGGCGTTCATCTGGGACAGTTCACG } \\
T\end{array}$ \\
\hline $\begin{array}{l}\text { NMDA- } \\
1 B\end{array}$ & KX016783.1 & $\begin{array}{l}\text { ACAGCCAAGACGAAGAAGAC / } \\
\text { CCGCTGTTCAGGATGACAGA }\end{array}$ & TGAGTTCATGGCCATCTCGGAGTC \\
\hline $\begin{array}{l}\text { NMDA- } \\
2 A\end{array}$ & KX016785.1 & $\begin{array}{l}\text { TCGGGTTCGTTCCCTTCAC / } \\
\text { TGATGCCGTCCGTGATAGAAG }\end{array}$ & TGAGACCATCCTTGCCAAGCACC \\
\hline $\begin{array}{l}\text { NMDA- } \\
2 B\end{array}$ & KX016786.1 & $\begin{array}{l}\text { GCAAGGGTCACCATCAGACA / } \\
\text { CGCTGTGAGCATGATGTAGGTA }\end{array}$ & $\begin{array}{c}\text { TGGAGAAACAACTTGAGGCCAATGG } \\
\text { A }\end{array}$ \\
\hline $\begin{array}{l}\text { NMDA-2- } \\
\text { like }\end{array}$ & KX016784.1 & $\begin{array}{l}\text { GCGTTGGAGCAGTTCATGTC / } \\
\text { GCCACATACTGACGGAAGTAC }\end{array}$ & CACGTTCCTGCTGTTGGGTTGTG \\
\hline KCNK2 & KU681437.1 & $\begin{array}{c}\text { GACGCCTTCTACTACTGCTTCATC / } \\
\text { GAGGGCGTTCTCCTTCTGTAG }\end{array}$ & CCCTCACTACCATTGGCTTCGGG \\
\hline KCNK1 & KU681438.1 & $\begin{array}{l}\text { TGGCGAACGACTCAACAAAG / } \\
\text { GTCCTGGCACTTAAGGATCTTC }\end{array}$ & СTCCTCCATCGGCATCAGGCAA \\
\hline$K C N Q 1$ & KU681453.1 & $\begin{array}{l}\text { GAGCCTCCTTGGGAAACCTATC / } \\
\text { CCGCTCCAGGAAGTTGTAGAC }\end{array}$ & CTCTCGCAGGGACGTCCGCTAC \\
\hline KCNQ2 & KU681452.1 & $\begin{array}{l}\text { GCTGCCATGTTGATCCAGTG / } \\
\text { CCACGTTGCTGTAGAGTTGAAG }\end{array}$ & TGTGGCGTTGTTATGCTGCAGATAA \\
\hline $\mathrm{KCNH} 2$ & KU681459.1 & $\begin{array}{c}\text { CACCGCGAGATCCTTTCAC / } \\
\text { CCTGATGTGGAGGCTGAGTAG }\end{array}$ & CATCTTCGAGACAGCGTCGCAGG \\
\hline $\mathrm{KCNH3}$ & KU681460.1 & $\begin{array}{l}\text { GAGGCGACGTACTTACCTCTATG / } \\
\text { AGTGGCGTACATGCAAGGATTC }\end{array}$ & АCTTCATCTCAAGAGGCTCGCTAGA \\
\hline $\mathrm{KCNH} 1$ & KU681458.1 & $\begin{array}{l}\text { GGTCACGTCACCACCATCATC / } \\
\text { CCGCACGTTGTTGAGCATTTC }\end{array}$ & ATGACCTCCGCCACCGCCAAGT \\
\hline KCNT1 & KU681454.1 & $\begin{array}{c}\text { CGTCCAGACCATGTTCAAGTTC / } \\
\text { AAGCGCATGTTGGACGACTG }\end{array}$ & TCCCCAACATCAAAATCATCACGGA \\
\hline CCAPr & KM349850.1 & $\begin{array}{l}\text { GCCCTTCTCCTCTCCAAATCAC / } \\
\text { GTCGGTGAGAACGCTGATGAG }\end{array}$ & $\begin{array}{c}\text { CCAGGACCAACTTCTTCATCATGCA } \\
\text { TCT }\end{array}$ \\
\hline vGluT & MK958905 & $\begin{array}{l}\text { GCGTTCGTGGACCTTCTAC / } \\
\text { TCAGCCACCCTGTAATGGAA }\end{array}$ & ATCACAGCCAACCTACTTCAGCGAG \\
\hline ChAT & MK958903 & $\begin{array}{l}\text { GGACCGCCTGGCTAAGTAC / } \\
\text { TCGCGGAGTCCCATAAGG }\end{array}$ & AGGCGGCGCTCAAGCTTCAGAC \\
\hline$v A C h T$ & MK958904 & $\begin{array}{l}\text { GCGTCAGCTGCTTCTTCCT / } \\
\text { CAGCAGTGCCGTGTCTATGAG }\end{array}$ & TTCGCCAGCAACTACTGGGTGTT \\
\hline$A C H E$ & MK958902 & $\begin{array}{l}\text { GGGCAACATGGGCATGTAC / } \\
\text { GGTCACCACCGAAGAATTCAATG }\end{array}$ & AGGCGCTGGCCATCAAGTGGATAC \\
\hline
\end{tabular}




\section{Chapter 5}

\section{The Cancer borealis cardiac ganglion as a model of compensation: Opportunities for future research}

Neural networks underlie rhythmic behaviors necessary for survival. Breathing, chewing, locomotion, and in the case of Cancer borealis and other crustaceans, heartbeat owe their origin to central pattern generators. Here we have used the cardiac ganglion to examine compensatory plasticity on the scale of minutes and on the scale of hours and with respect to membrane properties and mRNA expression changes, ultimately broadening our focus to consider how distinct different cell types are following an unknown history of physiological challenges and compensatory events. Below, we summarize the findings of the previous three chapters and suggest how the cardiac ganglion might be used to further advance our understanding of activity dependent compensation.

In chapter 2 we demonstrate that activity dependent gap junction plasticity occurs not due to depolarization per se but due to the asynchrony between neurons. Furthermore, whether coupling increases or decreases depends on how desynchronized the neurons are. Similar to plasticity in other systems (Haas et al., 2011a; Sevetson et al., 2017b; Welzel and Schuster, 2018; Fricker et al., 2020) this phenomenon is calcium dependent suggesting the effect may be generalizable. We find that when we allow stimuli to vary naturalistically and leverage this variability to evaluate other possible electrophysiological features, none better explain the change in coupling than activity timing. Finally, we show 
that a cells output becomes compromised when it is strongly coupled to a cell acting out of time with it, and if coupling is reduced, it will produce an output closer to its normal activity.

In chapter 3 we show that compensation to hyperexcitability extends beyond membrane properties. Within an hour there are changes in mRNA abundances and abundance relationships. Furthermore, we show that although excitability metrics are similar to control after one hour, as expected from previous evidence of rapid homeostatic compensation to hyperexcitability (Ransdell et al., 2012a; Lane et al., 2016), the cell has not reached steady state at this time. We find that after twenty-four hours there are physiological and molecular changes relative to one hour. Additionally, we note that excitability metrics suggest neurons fail to maintain compensation over this prolonged challenge, suggesting there may be limits to cell specific compensation or that network properties may be altered on a longer time scale.

In chapter 4 we demonstrate that despite substantial variability in mRNA expression within cell types (Schulz, 2006; Tobin et al., 2009), it is possible to recapitulate neuron cell identity from mRNA abundances even for neurons of the same ganglion. However, we find there are limits to this classification - no methodology we tested predicted cell type with perfect accuracy, even when we used a selection of physiologically relevant genes or differentially expressed contigs (the latter serving as a best-case scenario). Additionally, we find that while we are able to outperform chance for a classification task (i.e., correctly labeling cells given a set of labeled cells to learn from) and cluster like cells 
together (without labeling them), but that we are unable to correctly infer the number of cell types present in the data set.

Despite its long history, there are numerous questions the cardiac ganglion can be applied to. Here, we focus on those that build off the previous chapters and the past work of the Schulz lab.

\section{Coupling regulation and co-regulation}

The cardiac ganglion permits repeatable isolation of coupled neurons and allows for study of strong local coupling between paired LCs (LC 4 and 5 or LC 1 and 2) or weaker coupling between all LCs. Building on the results of chapter 2, an extension would be further characterization the electrophysiological properties which elicit coupling plasticity. We note that although desynchronization appears to be the sine qua non of activity dependent coupling the same delay applied with control or high amplitude results in depression or potentiation, respectively. Characterization of the interaction effects between activity features (e.g., mean depolarization, number of spikes, duty cycle, period, etc.) using simpler waveforms would be valuable in determining which features interact with timing.

Beyond varying the set of stimuli considered, expanding the scope to include the effects on membrane conductances would further a more holistic understanding of activity dependent compensation. We demonstrated that application of cadmium prevents plasticity. Calcium signaling has been implicated in the compensatory increase of $I_{H T K}$ following blockade of $I_{A}$ with 4aminopurine (Ransdell et al., 2013b) and evidence suggestive of relationship

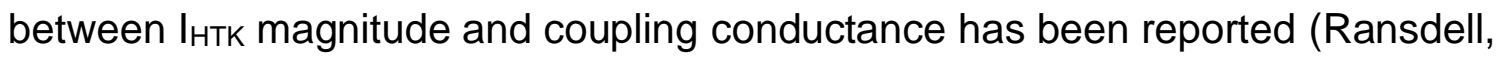


2013b). Either through identification of the molecular mechanisms of coupling potentiation and depression within the cardiac ganglion or by demonstrating alteration of one induces alteration of the other would be feasible next steps to establishing this system to effectively investigate coregulation of ionic and coupling properties.

\section{Cell and network level compensation}

Our dynamic clamp experiments did not permit biological compensation to pathological activity. Examining the changes in network activity in response to one or several pathologically active neurons would be a clear next step. Similar experiments have been attempted by using petroleum jelly wells filled with TEA containing saline to disrupt one, two, or three of the anterior large cells, but encountered difficulties with implementation. We propose instead a purely electrical manipulation. With voltage clamp a LC could be clamped to a loop of its own activity. The activity of the network would be monitored to determine if entrains to the perturbed cell thereby eliminating a difference in timing or whether coupling between LCs is altered. In a similar fashion, dynamic clamp could be used to mimic blockade of $I_{\text {HTK. }}$ Varying the strength of the disruption and measuring outward conductances, coupling, and activity in non-clamped cells one might reveal multi-faceted tuning relationships (between $I_{A}$, $I_{H T K}$, coupling, and small cell stimulation) more representative of those expected to be operating in vivo.

Dynamic clamp or voltage clamp would serve as an excellent tool to extend the research presented in chapter 3 . In the stomatogastric ganglion, multi- 
hour voltage clamp has been applied to study the preservation of mRNA correlations (Santin and Schulz, 2019a). We propose applying the same strategy to explore the plasticity of correlations. We have shown that mRNA abundances and correlations can be altered within one hour of TEA induced hyperexcitability. It is unclear whether this apparent difference in compensatory delay is an artifact of experimental design (i.e., that neurons in the stomatogastric ganglion have correlations labile at one hour but were not sampled at this time point), or if it represents differing time courses between neurons, loss of correlations rather than establishment of correlations, or if there is something fundamentally different about the response to too much activity relative to none. Voltage clamping two LCs is entirely feasible, but for more than a few hours would be challenging. We recommend any experimentalists interested in this line of inquiry to consider recording a cell's baseline activity, silencing the network using isotonic sucrose and then voltage clamping LC 5 with a reference electrode in LC 4 and LC 3. LC 4 will receive some stimulation through the strong local coupling; LC 3 will receive almost none. Using the recordings from each cell attenuation can be calculated if there is sufficient variability in non-clamped cell voltage to treat stimulation as a continuous variable. An alternate approach to this question would be to apply different blockers or different concentrations of a blocker to increase or decrease cells' activity. This approach would permit comparison of differing compensatory strategies to depolarizing and hyperpolarizing perturbations albeit in a less controlled manner.

\section{Estimating variability and nontarget effects}


Chapter 4 sought to establish to what extent one can recapitulate physiological cell identity from molecular abundances and abundance relationships. These systems, both the cardiac ganglion and the stomatogastric ganglion can be leveraged to address a vital methodological question - how much variability exists within identified neurons and how much might be attributable to effects such as seasonality (Lane, 2016), experimenter, or batch effects. Using the wealth of control physiology and molecular data collected for these systems we propose fitting a statistical model with these features included. Unfortunately, as these animals are wild caught from undisclosed locations there are many relevant features which one would be unable to include. Still, one could derive a more accurate depiction of which relationships between physiological measures are more or less likely to replicate. Furthermore, the definition of control distributions of physiological features would aid in generating and evaluating in silico models of these systems and producing reasonable priors for statistical modeling of future experiments. 


\section{BIBLIOGRAPHY}

Adobe Inc. (2019) Adobe Illustrator. Available at: https://adobe.com/products/illustrator.

Alcamí P, Pereda AE (2019) Beyond plasticity: the dynamic impact of electrical synapses on neural circuits. Nature Reviews Neuroscience 20:253-271.

Alexandrowicz J (1932) the Innervation of the heart of the Crustacea. I. Decapoda. Q J Microsc Sci.

Allen MJ, Godenschwege TA, Tanouye MA, Phelan P (2006) Making an escape: Development and function of the Drosophila giant fibre system. Seminars in Cell and Developmental Biology 17:31-41.

Andrews T (2019) M3Drop: Michaelis-Menten Modelling of Dropouts in single-cell RNASeq.

Arendt D, Musser JM, Baker CVH, Bergman A, Cepko C, Erwin DH, Pavlicev M, Schlosser G, Widder S, Laubichler MD, Wagner GP (2016) The origin and evolution of cell types. Nature Reviews Genetics 17:744-757.

Avise JC (1994) History of Molecular Phylogenetics. In: Molecular Markers, Natural History and Evolution. Boston, MA: Springer.

Benito E, Barco A (2015) The Neuronal Activity-Driven Transcriptome. Molecular Neurobiology 51:1071-1088.

Bennett MVL (1966a) Physiology of Electronic Junctions. Annals New York Academy of Sciences:509-539.

Bennett MVL (1966b) Physiology of Electronic Junctions. Annals of the New York Academy of Sciences 137:509-539.

Bikoff JB, Gabitto MI, Rivard AF, Drobac E, Machado TA, Miri A, Brenner-Morton S, Famojure E, Diaz C, Alvarez FJ, Mentis GZ, Jessell TM (2016) Spinal Inhibitory Interneuron Diversity Delineates Variant Motor Microcircuits. Cell 165:207-219.

Boldog E et al. (2018) Transcriptomic and morphophysiological evidence for a specialized human cortical GABAergic cell type. Nature Neuroscience 21:11851195.

Bray NL, Pimentel H, Melsted P, Pachter L (2016) Near-optimal probabilistic RNA-seq quantification. Nature Biotechnology 34:525-527.

Brennecke P, Anders S, Kim JK, Kołodziejczyk AA, Zhang X, Proserpio V, Baying B, Benes V, Teichmann SA, Marioni JC, Heisler MG (2013) Accounting for technical noise in single-cell RNA-seq experiments. Nature Methods 10.

Cadwell CR, Palasantza A, Jiang X, Berens P, Deng Q, Yilmaz M, Reimer J, Shen S, Bethge M, Tolias KF, Sandberg R, Tolias AS (2015) Electrophysiological, 
transcriptomic and morphologic profiling of single neurons using Patch-seq. Nature Biotechnology 34:199-203.

Cembrowski MS, Menon V (2018) Continuous Variation within Cell Types of the Nervous System. Trends in Neurosciences 41:337-348.

Cembrowski MS, Wang L, Sugino K, Shields BC, Spruston N (2016) Hipposeq: a comprehensive RNA-seq database of gene expression in hippocampal principal neurons. eLife 5:e14997.

Chung S, Weber F, Zhong P, Tan CL, Nguyen TN, Beier KT, Hörmann N, Chang W-C, Zhang Z, Do JP, Yao S, Krashes MJ, Tasic B, Cetin A, Zeng H, Knight ZA, Luo L, Dan $Y$ (2017) Identification of preoptic sleep neurons using retrograde labelling and gene profiling. Nature 545:477-481.

Cooke IM (2002) Reliable, responsive pacemaking and pattern generation with minimal cell numbers: The crustacean cardiac ganglion. Biological Bulletin 202:108-136.

Crocker A, Guan X-J, Murphy CT, Murthy M (2016) Cell-Type-Specific Transcriptome Analysis in the Drosophila Mushroom Body Reveals Memory-Related Changes in Gene Expression. Cell Reports 15:1580-1596.

Cruz-Bermudez ND, Marder E (2007) Multiple modulators act on the cardiac ganglion of the crab, Cancer borealis. Journal of Experimental Biology 210:2873-2884.

Cuevas-Diaz Duran R, Wei H, Wu JQ (2017) Single-cell RNA-sequencing of the brain. Clinical and Translational Medicine 6:20.

Curti S, O'Brien J (2016) Characteristics and plasticity of electrical synaptic transmission. BMC Cell Biology 17:13.

Davie K et al. (2018) A Single-Cell Transcriptome Atlas of the Aging Drosophila Brain. Cell 174:982-998.e20.

Deneris ES, Hobert O (2014) Maintenance of postmitotic neuronal cell identity. Nature Neuroscience 17:899-907.

Doyle JP, Dougherty JD, Heiman M, Schmidt EF, Stevens TR, Ma G, Bupp S, Shrestha P, Shah RD, Doughty ML, Gong S, Greengard P, Heintz N (2008) Application of a Translational Profiling Approach for the Comparative Analysis of CNS Cell Types. Cell 135:749-762.

Eberwine J, Yeh H, Miyashiro K, Cao Y, Nair S, Finnell R, Zettel M, Coleman P (1992) Analysis of gene expression in single live neurons. Proceedings of the National Academy of Sciences of the United States of America 89:3010-3014.

Edwards SM (2019) lemon: Freshing Up your "ggplot2" Plots. Available at: https://CRAN.R-project.org/package=lemon. 
Esumi S, Wu S-X, Yanagawa Y, Obata K, Sugimoto Y, Tamamaki N (2008) Method for single-cell microarray analysis and application to gene-expression profiling of GABAergic neuron progenitors. Neuroscience Research 60:439-451.

Farrell JA, Wang Y, Riesenfeld SJ, Shekhar K, Regev A, Schier AF (2018) Single-cell reconstruction of developmental trajectories during zebrafish embryogenesis. Science (New York, NY) 360:eaar3131.

Firke S (2020) janitor: Simple Tools for Examining and Cleaning Dirty Data. Available at: https://CRAN.R-project.org/package=janitor.

Fox J, Weisberg S (2019) An R Companion to Applied Regression, Third. Thousand Oaks CA: Sage. Available at:

https://socialsciences.mcmaster.ca/jfox/Books/Companion/.

Freudenstein J V, Broe MB, Folk RA, Sinn BT (2017) Biodiversity and the Species Concept-Lineages are not Enough. Systematic biology 66:644-656.

Fricker BA, Heckman EL, Cunningham PC, Wang H, Haas JS (2020) Activity-dependent long-term potentiation of electrical synapses in the mammalian thalamus. Journal of Neurophysiology:36.

from Jed Wing MKuhnC, Weston S, Williams A, Keefer C, Engelhardt A, Cooper T, Mayer Z, Kenkel B, the R Core Team, Benesty M, Lescarbeau R, Ziem A, Scrucca L, Tang Y, Candan C, Hunt. T (2018) caret: Classification and Regression Training.

Furshpan EJ, Potter DD (1959) Transmission At the Giant Motor Synapses of the Crayfish. J Physiol (1959) 45:289-325.

Gerschenfelds M (1984) HORIZONTAL CELLS OF TURTLE RETINA. The Journal of Neuroscience 4:12.

Gokce O, Stanley GM, Treutlein B, Neff NF, Camp JG, Malenka RC, Rothwell PE, Fuccillo M V, Südhof TC, Quake SR (2016) Cellular Taxonomy of the Mouse Striatum as Revealed by Single-Cell RNA-Seq. Cell reports 16:1126-1137.

Guerra L, McGarry LM, Robles V, Bielza C, Larrañaga P, Yuste R (2011) Comparison between supervised and unsupervised classifications of neuronal cell types: $A$ case study. Developmental Neurobiology 71:71-82.

Gutekunst J, Andriantsoa R, Falckenhayn C, Hanna K, Stein W, Rasamy J, Lyko F (2018) Clonal genome evolution and rapid invasive spread of the marbled crayfish. Nature ecology \& evolution 2:567-573.

Gutierrez GJ, O'Leary T, Marder E (2013) Multiple Mechanisms Switch an Electrically Coupled, Synaptically Inhibited Neuron between Competing Rhythmic Oscillators. Neuron 77:845-858. 
Haas BJ et al. (2013) De novo transcript sequence reconstruction from RNA-seq using the Trinity platform for reference generation and analysis. Nature protocols 8:1494-1512.

Haas JS, Greenwald CM, Pereda AE (2016) Activity-dependent plasticity of electrical synapses: increasing evidence for its presence and functional roles in the mammalian brain. BMC Cell Biology 17 Available at:

http://bmccellbiol.biomedcentral.com/articles/10.1186/s12860-016-0090-z [Accessed June 18, 2019].

Haas JS, Landisman CE (2012) Bursts modify electrical synaptic strength. Brain Research 1487:140-149.

Haas JS, Zavala B, Landisman CE (2011a) Activity-dependent long-term depression of electrical synapses. Science 334:389-393.

Haas JS, Zavala B, Landisman CE (2011b) Activity-Dependent Long-Term Depression of Electrical Synapses. 389:389-394.

Haas JS, Zavala B, Landisman CE (2011c) Activity-dependent long-term depression of electrical synapses. Science 334:389-393.

Hamood AW, Haddad SA, Otopalik AG, Rosenbaum P, Marder E (2015) Quantitative Reevaluation of the Effects of Short- and Long-Term Removal of Descending Modulatory Inputs on the Pyloric Rhythm of the Crab, Cancer borealis. eneuro 2:ENEURO.0058-14.2015.

Harris RM, Kao H-Y, Alarcon JM, Hofmann HA, Fenton AA (2019) Hippocampal transcriptomic responses to enzyme-mediated cellular dissociation.

Hippocampus:hipo.23095.

Harris TW et al. (2014) WormBase 2014: new views of curated biology. Nucleic Acids Research 42:D789-D793.

He LS, Rue MCP, Morozova EO, Powell DJ, James EJ, Kar M, Marder E (2020) Rapid adaptation to elevated extracellular potassium in the pyloric circuit of the crab, Cancer borealis. Journal of Neurophysiology 123:2075-2089.

Ho H, Both M De, Siniard A, Sharma S, Notwell JH, Wallace M, Leone DP, Nguyen A, Zhao E, Lee H, Zwilling D, Thompson KR, Braithwaite SP, Huentelman M, Portmann T (2018) A Guide to Single-Cell Transcriptomics in Adult Rodent Brain: The Medium Spiny Neuron Transcriptome Revisited. Frontiers in Cellular Neuroscience 12:159.

Hobert O, Carrera I, Stefanakis N (2010) The molecular and gene regulatory signature of a neuron. Trends in Neurosciences 33:435-445.

Hobert O, Glenwinkel L, White J (2016) Revisiting Neuronal Cell Type Classification in Caenorhabditis elegans. Current biology : CB 26:R1197-R1203. 
Hooper SL, O'Neil MB, Wagner R, Ewer J, Golowasch J, Marder E (1986) The innervation of the pyloric region of the crab, Cancer borealis: homologous muscles in decapod species are differently innervated. Journal of comparative physiology A, Sensory, neural, and behavioral physiology 159:227-240.

Kelley KW, Nakao-Inoue H, Molofsky A V., Oldham MC (2018) Variation among intact tissue samples reveals the core transcriptional features of human CNS cell classes. Nature Neuroscience 21:1171-1184.

Khorkova O, Golowasch J (2007a) Neuromodulators, not activity, control coordinated expression of ionic currents. Journal of Neuroscience 27:8709-8718.

Khorkova O, Golowasch J (2007b) Neuromodulators, Not Activity, Control Coordinated Expression of Ionic Currents. Journal of Neuroscience 27:8709-8718.

Kilman V, Marder E (1996) Ultrastructure of the stomatogastric ganglion neuropil of the crab, Cancer borealis. The Journal of Comparative Neurology 374:362-375.

Kim D-W, Yao Z, Graybuck LT, Kim TK, Nguyen TN, Smith KA, Fong O, Yi L, Koulena N, Pierson N, Shah S, Lo L, Pool A-H, Oka Y, Pachter L, Cai L, Tasic B, Zeng H, Anderson DJ (2019) Multimodal Analysis of Cell Types in a Hypothalamic Node Controlling Social Behavior. Cell 179:713-728.e17.

Konstantinidis KT, Ramette A, Tiedje JM (2006) The bacterial species definition in the genomic era. Philosophical Transactions of the Royal Society B: Biological Sciences 361:1929-1940.

Kothmann WW, Trexler EB, Whitaker CM, Li W, Massey SC, O'Brien J (2012) Nonsynaptic NMDA Receptors Mediate Activity-Dependent Plasticity of Gap Junctional Coupling in the All Amacrine Cell Network. Journal of Neuroscience 32:6747-6759.

Lane BJ, Kick DR, Wilson DK, Nair SS, Schulz DJ (2018) Dopamine maintains network synchrony via direct modulation of gap junctions in the crustacean cardiac ganglion. eLife 7 Available at: https://elifesciences.org/articles/39368 [Accessed September 1, 2020].

Lane BJ (MU-S (2016) Homeostatic compensation and neuromodulation maintain synchronized motor neuron activity in the crustacean cardiac ganglion.

Lane BJ, Samarth P, Ransdell JL, Nair SS, Schulz DJ (2016) Synergistic plasticity of intrinsic conductance and electrical coupling restores synchrony in an intact motor network. eLife 5:1-23.

Li G-W, Xie XS (2011) Central dogma at the single-molecule level in living cells. Nature 475:308-315.

Li H, Horns F, Wu B, Xie Q, Li J, Li T, Luginbuhl DJ, Quake SR, Luo L (2017) Classifying Drosophila Olfactory Projection Neuron Subtypes by Single-Cell RNA Sequencing. Cell 171:1206-1220.e22. 
Llorens-Bobadilla E, Zhao S, Baser A, Saiz-Castro G, Zwadlo K, Martin-Villalba A (2015) Single-Cell Transcriptomics Reveals a Population of Dormant Neural Stem Cells that Become Activated upon Brain Injury. Cell Stem Cell 17:329-340.

Long MA, Deans MR, Paul DL, Connors BW (2002) Rhythmicity without synchrony in the electrically uncoupled inferior olive. The Journal of neuroscience : the official journal of the Society for Neuroscience 22:10898-10905.

Luo L, Callaway EM, Svoboda K (2018) Genetic Dissection of Neural Circuits: A Decade of Progress. Neuron 98:256-281.

Luther JA, Robie AA, Yarotsky J, Reina C, Marder E, Golowasch J (2003) Episodic Bouts of Activity Accompany Recovery of Rhythmic Output By a Neuromodulator- and Activity-Deprived Adult Neural Network. Journal of Neurophysiology 90:2720-2730.

MacLean JN, Zhang Y, Goeritz ML, Casey R, Oliva R, Guckenheimer J, Harris-Warrick RM (2005) Activity-Independent Coregulation of $I_{\mathrm{A}}$ and $I_{\mathrm{h}}$ in Rhythmically Active Neurons. Journal of Neurophysiology 94:3601-3617.

MacLean JN, Zhang Y, Johnson BR, Harris-Warrick RM (2003) Activity-Independent Homeostasis in Rhythmically Active Neurons. Neuron 37:109-120.

Macosko EZ, Basu A, Satija R, Nemesh J, Shekhar K, Goldman M, Tirosh I, Bialas AR, Kamitaki N, Martersteck EM, Trombetta JJ, Weitz DA, Sanes JR, Shalek AK, Regev A, McCarroll SA (2015) Highly Parallel Genome-wide Expression Profiling of Individual Cells Using Nanoliter Droplets. Cell 161:1202-1214.

Marder E (1976) Cholinergic motor neurones in the stomatogastric system of the lobster. The Journal of Physiology 257:63-86.

Marder E (2011) Variability, compensation, and modulation in neurons and circuits. Proceedings of the National Academy of Sciences 108:15542-15548.

Marder E, Bucher D (2007) Understanding circuit dynamics using the stomatogastric nervous system of lobsters and crabs. Annual review of physiology 69:291-316.

Marder E, Gutierrez GJ, Nusbaum MP (2017) Complicating connectomes: Electrical coupling creates parallel pathways and degenerate circuit mechanisms. Developmental Neurobiology 77:597-609.

Martin M (2011) Cutadapt removes adapter sequences from high-throughput sequencing reads. EMBnet.journal 17:10.

Masland RH (2004) Neuronal cell types. Current Biology 14:R497-R500.

Mazerolle MJ (2019) AICcmodavg: Model selection and multimodel inference based on (Q)AIC(c). Available at: https://cran.r-project.org/package=AICcmodavg.

McElreath R (2016) Statistical Rethinking: a Bayesian course with examples in R and Stan, 1st ed. CRC Press. 
Mehta P, Kreeger L, Wylie DC, Pattadkal JJ, Lusignan T, Davis MJ, Turi GF, Li W-K, Whitmire MP, Chen Y, Kajs BL, Seidemann E, Priebe NJ, Losonczy A, Zemelman B V. (2019) Functional Access to Neuron Subclasses in Rodent and Primate Forebrain. Cell Reports 26:2818-2832.e8.

Mi H, Muruganujan A, Thomas PD (2012) PANTHER in 2013: modeling the evolution of gene function, and other gene attributes, in the context of phylogenetic trees. Nucleic Acids Research 41:D377-D386.

Mito M, Kadota M, Tanaka K, Furuta Y, Abe K, Iwasaki S, Nakagawa S (2018) Cell Type-Specific Survey of Epigenetic Modifications by Tandem Chromatin Immunoprecipitation Sequencing. Scientific Reports 8:1143.

Moroz LL, Kohn AB (2013) Single-Neuron Transcriptome and Methylome Sequencing for Epigenomic Analysis of Aging. In: Methods in molecular biology (Clifton, N.J.), pp 323-352.

Müller K (2017) here: A Simpler Way to Find Your Files. Available at: https://CRAN.Rproject.org/package=here.

Nakase T, Naus CCG (2004) Gap junctions and neurological disorders of the central nervous system. Biochimica et Biophysica Acta (BBA) - Biomembranes 1662:149-158.

Northcutt AJ, Lett KM, Garcia VB, Diester CM, Lane BJ, Marder E, Schulz DJ (2016a) Deep sequencing of transcriptomes from the nervous systems of two decapod crustaceans to characterize genes important for neural circuit function and modulation. BMC Genomics 17 Available at: http://bmcgenomics.biomedcentral.com/articles/10.1186/s12864-016-3215-z [Accessed January 31, 2019].

Northcutt AJ, Lett KM, Garcia VB, Diester CM, Lane BJ, Marder E, Schulz DJ (2016b) Deep sequencing of transcriptomes from the nervous systems of two decapod crustaceans to characterize genes important for neural circuit function and modulation. BMC Genomics 17:868.

O'Leary T, Williams AH, Caplan JS, Marder E (2013a) Correlations in ion channel expression emerge from homeostatic tuning rules. Proceedings of the National Academy of Sciences 110:E2645-E2654.

O'Leary T, Williams AH, Caplan JS, Marder E (2013b) Correlations in ion channel expression emerge from homeostatic tuning rules. Proceedings of the National Academy of Sciences 110:E2645-E2654.

O'Leary T, Williams AH, Franci A, Marder E (2014a) Cell Types, Network Homeostasis, and Pathological Compensation from a Biologically Plausible lon Channel Expression Model. Neuron 82:809-821.

O'Leary T, Williams AHH, Franci A, Marder E, O'Leary T, Williams AHH, Franci A, Marder E (2014b) Cell Types, Network Homeostasis, and Pathological 
Compensation from a Biologically Plausible Ion Channel Expression Model. Neuron 82:809-821.

Olivera-Martinez I, Schurch N, Li RA, Song J, Halley PA, Das RM, Burt DW, Barton GJ, Storey KG (2014) Major transcriptome re-organisation and abrupt changes in signalling, cell cycle and chromatin regulation at neural differentiation in vivo. Development 141:3266-3276.

Oshima A, Tani K, Fujiyoshi Y (2016) Atomic structure of the innexin-6 gap junction channel determined by cryo-EM. Nat Commun 7:13681.

Parmhans N, Sajgo S, Niu J, Luo W, Badea TC (2018) Characterization of retinal ganglion cell, horizontal cell, and amacrine cell types expressing the neurotrophic receptor tyrosine kinase Ret. Journal of Comparative Neurology 526:742-766.

Paul A, Crow M, Raudales R, He M, Gillis J, Huang ZJ (2017) Transcriptional Architecture of Synaptic Communication Delineates GABAergic Neuron Identity. Cell 171:522-539.e20.

Pedersen TL (2019) patchwork: The Composer of Plots. Available at: https://CRAN.Rproject.org/package=patchwork.

Peng Y-R, Shekhar K, Yan W, Herrmann D, Sappington A, Bryman GS, van Zyl T, Do MTriH, Regev A, Sanes JR (2019) Molecular Classification and Comparative Taxonomics of Foveal and Peripheral Cells in Primate Retina. Cell 176:12221237.e22.

Perez-Ortin JE (2008) Genomics of mRNA turnover. Briefings in Functional Genomics and Proteomics 6:282-291.

Pimentel H, Bray NL, Puente S, Melsted P, Pachter L (2017) Differential analysis of RNA-seq incorporating quantification uncertainty. Nature Methods 14:687-690.

Pinheiro J, Bates D, DebRoy S, Sarkar D, R Core Team (2020) nlme: Linear and Nonlinear Mixed Effects Models. Available at: https://CRAN.Rproject.org/package=nlme.

Poulin J-F, Tasic B, Hjerling-Leffler J, Trimarchi JM, Awatramani R (2016) Disentangling neural cell diversity using single-cell transcriptomics. Nature neuroscience 19:1131-1141.

Prinz AA, Bucher D, Marder E (2004) Similar network activity from disparate circuit parameters. Nature neuroscience 7:1345-1352.

R Core Team (2020) R: A Language and Environment for Statistical Computing. Vienna, Austria: R Foundation for Statistical Computing. Available at: https://www.Rproject.org/.

R Core Team, Team RDC (2008) R: A Language and Environment for Statistical Computing. 
Raj A, van Oudenaarden A (2008) Nature, Nurture, or Chance: Stochastic Gene Expression and Its Consequences. Cell 135:216-226.

Ransdell $\mathrm{JL}$ (2013a) Motor neuron output in the crustacean cardiac ganglion is organized and maintained by homeostatic conductance relationships.

Ransdell JL, Faust TB, Schulz DJ (2010) Correlated Levels of mRNA and Soma Size in Single Identified Neurons: Evidence for Compartment-specific Regulation of Gene Expression. Frontiers in Molecular Neuroscience 3 Available at: http://journal.frontiersin.org/article/10.3389/fnmol.2010.00116/abstract [Accessed January 31, 2019].

Ransdell JL (MU-S (2013b) Motor neuron output in the crustacean cardiac ganglion is organized and maintained by homeostatic conductance relationships.

Ransdell JL, Nair SS, Schulz DJ (2012a) Rapid homeostatic plasticity of intrinsic excitability in a central pattern generator network stabilizes functional neural network output. The Journal of neuroscience : the official journal of the Society for Neuroscience 32:9649-9658.

Ransdell JL, Nair SS, Schulz DJ (2012b) Rapid Homeostatic Plasticity of Intrinsic Excitability in a Central Pattern Generator Network Stabilizes Functional Neural Network Output. Journal of Neuroscience 32:9649-9658.

Ransdell JL, Nair SS, Schulz DJ (2013a) Neurons within the Same Network Independently Achieve Conserved Output by Differentially Balancing Variable Conductance Magnitudes. Journal of Neuroscience 33:9950-9956.

Ransdell JL, Nair SS, Schulz DJ (2013b) Neurons within the Same Network Independently Achieve Conserved Output by Differentially Balancing Variable Conductance Magnitudes. Journal of Neuroscience 33:9950-9956.

Reuter JA, Spacek D V., Snyder MP (2015) High-Throughput Sequencing Technologies. Molecular Cell 58:586-597.

Rizzetto S, Eltahla AA, Lin P, Bull R, Lloyd AR, Ho JWK, Venturi V, Luciani F (2017) Impact of sequencing depth and read length on single cell RNA sequencing data of T cells. Scientific Reports 7:12781.

Robinson A (2016) equivalence: Provides Tests and Graphics for Assessing Tests of Equivalence. Available at: https://CRAN.R-project.org/package=equivalence.

Robinson D, Hayes A (2020) broom: Convert Statistical Analysis Objects into Tidy Tibbles. Available at: https://CRAN.R-project.org/package=broom.

Rosenberg AB, Roco CM, Muscat RA, Kuchina A, Sample P, Yao Z, Graybuck LT, Peeler DJ, Mukherjee S, Chen W, Pun SH, Sellers DL, Tasic B, Seelig G (2018) Single-cell profiling of the developing mouse brain and spinal cord with split-pool barcoding. Science 360:176-182. 
Sanes JR, Masland RH (2015) The Types of Retinal Ganglion Cells: Current Status and Implications for Neuronal Classification. Annual Review of Neuroscience 38:221246.

Santin JM, Schulz DJ (2019a) Membrane Voltage Is a Direct Feedback Signal That Influences Correlated lon Channel Expression in Neurons. Current Biology 29:1683-1688.e2.

Santin JM, Schulz DJ (2019b) Membrane Voltage Is a Direct Feedback Signal That Influences Correlated lon Channel Expression in Neurons. Current Biology 29:1683-1688.e2.

Schulz DJ (2006) Plasticity and stability in neuronal output via changes in intrinsic excitability: it's what's inside that counts. Journal of Experimental Biology 209:4821-4827.

Schulz DJ, Goaillard J-M, Marder E (2006a) Variable channel expression in identified single and electrically coupled neurons in different animals. Nature Neuroscience 9:356-362.

Schulz DJ, Goaillard J-M, Marder E (2006b) Variable channel expression in identified single and electrically coupled neurons in different animals. Nature neuroscience 9:356-362.

Schulz DJ, Goaillard J-M, Marder EE (2007a) Quantitative expression profiling of identified neurons reveals cell-specific constraints on highly variable levels of gene expression. Proceedings of the National Academy of Sciences 104:1318713191.

Schulz DJ, Goaillard J-M, Marder EE (2007b) Quantitative expression profiling of identified neurons reveals cell-specific constraints on highly variable levels of gene expression. Proceedings of the National Academy of Sciences 104:1318713191.

Schulz DJ, Goaillard J-M, Marder EE (2007c) Quantitative expression profiling of identified neurons reveals cell-specific constraints on highly variable levels of gene expression. Proceedings of the National Academy of Sciences of the United States of America 104:13187-13191.

Schulz DJ, Lane BJ (2017) Homeostatic plasticity of excitability in crustacean central pattern generator networks. Current Opinion in Neurobiology 43:7-14.

Sekula M, Datta S, and Susmita Datta (2017) optCluster: Determine Optimal Clustering Algorithm and Number of Clusters.

Sevetson J, Fittro S, Heckman E, Haas JS (2017a) A calcium-dependent pathway underlies activity-dependent plasticity of electrical synapses in the thalamic reticular nucleus: Calcium and plasticity of electrical synapses. The Journal of Physiology 595:4417-4430. 
Sevetson J, Fittro S, Heckman E, Haas JS (2017b) A calcium-dependent pathway underlies activity-dependent plasticity of electrical synapses in the thalamic reticular nucleus. The Journal of Physiology 595:4417-4430.

Sha Y, Phan JH, Wang MD (2015) Effect of low-expression gene filtering on detection of differentially expressed genes in RNA-seq data. Conference proceedings : . Annual International Conference of the IEEE Engineering in Medicine and Biology Society IEEE Engineering in Medicine and Biology Society Annual Conference 2015:6461-6464.

Sheng K, Cao W, Niu Y, Deng Q, Zong C (2017) Effective detection of variation in single-cell transcriptomes using MATQ-seq. Nature Methods 14:267-270.

Shin J, Ming G, Song H (2014) Decoding neural transcriptomes and epigenomes via high-throughput sequencing. Nature neuroscience 17:1463-1475.

Shrestha BR, Chia C, Wu L, Kujawa SG, Liberman MC, Goodrich L V. (2018) Sensory Neuron Diversity in the Inner Ear Is Shaped by Activity. Cell 174:1229-1246.e17.

Shruti S, Schulz DJ, Lett KM, Marder E (2014) Electrical coupling and innexin expression in the stomatogastric ganglion of the crab Cancer borealis. Journal of neurophysiology 112:2946-2958.

Sievert C (2018) plotly for R. Available at: https://plotly-r.com.

Slowikowski K (2020) ggrepel: Automatically Position Non-Overlapping Text Labels with "ggplot2." Available at: https://CRAN.R-project.org/package=ggrepel.

Södersten E, Toskas K, Rraklli V, Tiklova K, Björklund ÅK, Ringnér M, Perlmann T, Holmberg J (2018) A comprehensive map coupling histone modifications with gene regulation in adult dopaminergic and serotonergic neurons. Nature Communications 9:1226.

Spitzer NC (2017) Neurotransmitter Switching in the Developing and Adult Brain. Annual Review of Neuroscience 40:1-19.

Spray DC, Harris AL, Bennett MVL (1979) Voltage Dependence of Junctional Conductance in Early Amphibian Embryos. Science 204.

Stevens CF (1998) Neuronal diversity: too many cell types for comfort? Current biology: CB 8:R708-10.

Stimberg M, Brette R, Goodman DF (2019) Brian 2, an intuitive and efficient neural simulator. eLife 8 Available at: https://elifesciences.org/articles/47314 [Accessed March 20, 2021].

Sugino K, Clark E, Schulmann A, Shima Y, Wang L, Hunt DL, Hooks BM, Tränkner D, Chandrashekar J, Picard S, Lemire AL, Spruston N, Hantman AW, Nelson SB (2019) Mapping the transcriptional diversity of genetically and anatomically defined cell populations in the mouse brain. eLife 8 . 
Swensen AM (2005) Robustness of Burst Firing in Dissociated Purkinje Neurons with Acute or Long-Term Reductions in Sodium Conductance. Journal of Neuroscience 25:3509-3520.

Syekirin S (2018) readABF: Loads Axon Binary Files. Available at: https://CRAN.Rproject.org/package=readABF.

Syekirin S (2020) readABF: Loads Axon Binary Files. Available at: https://CRAN.Rproject.org/package=readABF.

Symmons O, Raj A (2016) What's Luck Got to Do with It: Single Cells, Multiple Fates, and Biological Nondeterminism. Molecular Cell 62:788-802.

Tang F, Barbacioru C, Wang Y, Nordman E, Lee C, Xu N, Wang X, Bodeau J, Tuch BB, Siddiqui A, Lao K, Surani MA (2009) mRNA-Seq whole-transcriptome analysis of a single cell. Nature Methods 6:377-382.

Tasic B et al. (2016) Adult mouse cortical cell taxonomy revealed by single cell transcriptomics. Nature neuroscience 19:335-346.

Tasic B (2018) Single cell transcriptomics in neuroscience: cell classification and beyond. Current Opinion in Neurobiology 50:242-249.

Tasic B et al. (2018) Shared and distinct transcriptomic cell types across neocortical areas. Nature 563:72-78.

Tasic B, Levi BP, Menon V (2017) Single-Cell Transcriptomic Characterization of Vertebrate Brain Composition, Development, and Function. In: Decoding Neural Circuit Structure and Function, pp 437-468. Cham: Springer International Publishing.

Temporal S, Lett KM, Schulz DJ (2014a) Activity-Dependent Feedback Regulates Correlated Ion Channel mRNA Levels in Single Identified Motor Neurons. Current Biology 24:1899-1904.

Temporal S, Lett KM, Schulz DJ (2014b) Activity-dependent feedback regulates correlated ion channel mRNA levels in single identified motor neurons. Current Biology 24:1899-1904.

Tessmar-Raible K, Raible F, Christodoulou F, Guy K, Rembold M, Hausen H, Arendt D (2007) Conserved sensory-neurosecretory cell types in annelid and fish forebrain: insights into hypothalamus evolution. Cell 129:1389-1400.

Thoby-Brisson M, Simmers J (1998) Neuromodulatory Inputs Maintain Expression of a Lobster Motor Pattern-Generating Network in a Modulation-Dependent State: Evidence from Long-Term Decentralization In Vitro. The Journal of Neuroscience 18:2212-2225.

Thoby-Brisson M, Simmers J (2000) Transition to Endogenous Bursting After Long-Term Decentralization Requires De Novo Transcription in a Critical Time Window. Journal of Neurophysiology 84:596-599. 
Tietjen I, Rihel JM, Cao Y, Koentges G, Zakhary L, Dulac C (2003) Single-cell transcriptional analysis of neuronal progenitors. Neuron 38:161-175.

Tobin A-E, Cruz-Bermúdez ND, Marder E, Schulz DJ (2009) Correlations in ion channel mRNA in rhythmically active neurons. PloS one 4:e6742.

Tomer R, Denes AS, Tessmar-Raible K, Arendt D (2010) Profiling by Image Registration Reveals Common Origin of Annelid Mushroom Bodies and Vertebrate Pallium. Cell 142:800-809.

Tosches MA, Laurent G (2019) Evolution of neuronal identity in the cerebral cortex. Current Opinion in Neurobiology 56:199-208.

Trapnell C (2015) Defining cell types and states with single-cell genomics. Genome Research 25:1491-1498.

Turrigiano G (2012) Homeostatic Synaptic Plasticity: Local and Global Mechanisms for Stabilizing Neuronal Function. Cold Spring Harbor Perspectives in Biology 4:a005736.

Turrigiano G, LeMasson G, Marder E (1995) Selective regulation of current densities underlies spontaneous changes in the activity of cultured neurons. The Journal of Neuroscience 15:3640-3652.

Turrigiano GG, Leslie KR, Desai NS, Rutherford LC, Nelson SB (1998) Activitydependent scaling of quantal amplitude in neocortical neurons. Nature 391:892896.

Turrigiano GG, Marder E (1993) Modulation of identified stomatogastric ganglion neurons in primary cell culture. Journal of Neurophysiology 69:1993-2002.

Usoskin D, Furlan A, Islam S, Abdo H, Lönnerberg P, Lou D, Hjerling-Leffler J, Haeggström J, Kharchenko O, Kharchenko P V, Linnarsson S, Ernfors P (2015) Unbiased classification of sensory neuron types by large-scale single-cell RNA sequencing. Nature neuroscience 18:145-153.

van den Brink SC, Sage F, Vértesy Á, Spanjaard B, Peterson-Maduro J, Baron CS, Robin C, van Oudenaarden A (2017) Single-cell sequencing reveals dissociationinduced gene expression in tissue subpopulations. Nature methods 14:935-936.

Vaughan D, Dancho M (2018) furrr: Apply Mapping Functions in Parallel using Futures. Available at: https://CRAN.R-project.org/package $=$ furrr.

Vervaeke K, LÖrincz A, Gleeson P, Farinella M, Nusser Z, Silver RA (2010) Rapid Desynchronization of an Electrically Coupled Interneuron Network with Sparse Excitatory Synaptic Input. Neuron 67:435-451.

Wagner A, Regev A, Yosef N (2016) Revealing the vectors of cellular identity with single-cell genomics. Nature Biotechnology 34:1145-1160. 
Wang S, Borst A, Zaslavsky N, Tishby N, Segev I (2017) Efficient encoding of motion is mediated by gap junctions in the fly visual system Graham LJ, ed. PLOS Computational Biology 13:e1005846.

Wang Y, Ni T, Wang W, Liu F (2018) Gene transcription in bursting: a unified mode for realizing accuracy and stochasticity. Biological Reviews.

Wang Z, Neely R, Landisman CE (2015) Activation of Group I and Group II Metabotropic Glutamate Receptors Causes LTD and LTP of Electrical Synapses in the Rat Thalamic Reticular Nucleus. Journal of Neuroscience 35:7616-7625.

Watanabe A (1958) The Interaction of Electrical Activity Among Neurons of Lobster Cardiac Ganglion. Japanese Journal of Physiology:305-318.

Weimann JM, Marder E (1994) Switching neurons are integral members of multiple oscillatory networks. Current Biology 4:896-902.

Weimann JM, Meyrand P, Marder E (1991) Neurons that form multiple pattern generators: identification and multiple activity patterns of gastric/pyloric neurons in the crab stomatogastric system. Journal of neurophysiology 65:111-122.

Welzel G, Schuster S (2018) Long-term potentiation in an innexin-based electrical synapse. Scientific Reports 8 Available at:

http://www.nature.com/articles/s41598-018-30966-w [Accessed January 31, 2019].

Whitaker KW, Neumeister H, Huffman LS, Kidd CE, Preuss T, Hofmann HA (2011) Serotonergic modulation of startle-escape plasticity in an African cichlid fish: a single-cell molecular and physiological analysis of a vital neural circuit. Journal of neurophysiology 106:127-137.

Wickham H et al. (2019) Welcome to the tidyverse. Journal of Open Source Software 4:1686.

Wickham H, Bryan J (2019) readxl: Read Excel Files. Available at: https://CRAN.Rproject.org/package=readxl.

Wilke CO (2019) cowplot: Streamlined Plot Theme and Plot Annotations for "ggplot2." Available at: https://CRAN.R-project.org/package=cowplot.

Williams AH, Kwiatkowski MA, Mortimer AL, Marder E, Zeeman ML, Dickinson PS (2013) Animal-to-animal variability in the phasing of the crustacean cardiac motor pattern: an experimental and computational analysis. Journal of Neurophysiology 109:2451-2465.

Xu C, Su Z (2015) Identification of cell types from single-cell transcriptomes using a novel clustering method. Bioinformatics 31:1974-1980.

Zeileis A, Grothendieck G (2005) zoo: S3 Infrastructure for Regular and Irregular Time Series. Journal of Statistical Software 14:1-27. 
Zeisel A, Muñoz-Manchado AB, Codeluppi S, Lönnerberg P, La Manno G, Juréus A, Marques S, Munguba H, He L, Betsholtz C, Rolny C, Castelo-Branco G, HjerlingLeffler J, Linnarsson S (2015) Brain structure. Cell types in the mouse cortex and hippocampus revealed by single-cell RNA-seq. Science (New York, NY) 347:1138-1142.

Zeng H, Sanes JR (2017) Neuronal cell-type classification: challenges, opportunities and the path forward. Nature Reviews Neuroscience 18:530-546.

Zhou T, Matsunami H (2018) Lessons from single-cell transcriptome analysis of oxygensensing cells. Cell and Tissue Research 372:403-415.

Ziegenhain C, Vieth B, Parekh S, Reinius B, Guillaumet-Adkins A, Smets M, Leonhardt $\mathrm{H}$, Heyn H, Hellmann I, Enard W (2017) Comparative Analysis of Single-Cell RNA Sequencing Methods. Molecular Cell 65:631-643.e4. 


\section{VITA}

I was born in Springfield, Missouri to Beth and Bob Kick and grew up with my older sister Laura. I attended Central High School completing the International Baccalaureate program before studying at Truman State University.

I gravitated towards research early, volunteering in Dr. Laszlo Kovac's lab prior to beginning my undergraduate degree. During my sophomore year, Dr. Brent Buckner encouraged me to apply for an NSF REU at University of Missouri-Columbia. I was accepted and worked in Dr. Christian Lorson's lab evaluating disease progression in a spinal muscular atrophy mouse model. Encouraged by this experience I sought out a second NSF REU position with Dr. Rahul Kanadia at the University of Connecticut studying splicing in retina. During my final year I worked in the lab of Dr. Diane JanickBuckner and Dr. Brent Buckner implementing an immunohistochemistry assay and prototyping a hydroponics system to aid in screening maize mutants for root morphology changes.

Following graduation from Truman with a Bachelor of Science I was accepted into the University of Missouri's PhD graduate program in the division of biological sciences. I have been funded both though an NIH T32 Training Grant and through teaching assistantship.

Before joining Dr. David Schulz' lab I rotated with Dr. Bing Zhang, Dr. Lorin Milescu, both of whom have continued to foster my growth as a scientist since. The Schulz lab has been a phenomenal community to be a part of and enabled me to explore regulation of CPG properties from expression to output.

I have accepted a postdoctoral position in the lab of Dr. Jacob Washburn at USDA-ARS, where I will apply machine learning and statistical methods to predict and 
model maize growth based on genetic and environmental factors. I intend to spend my career in government or industry. 